Mondes réels, mondes virtuels

Jean Soumagne (dir.)

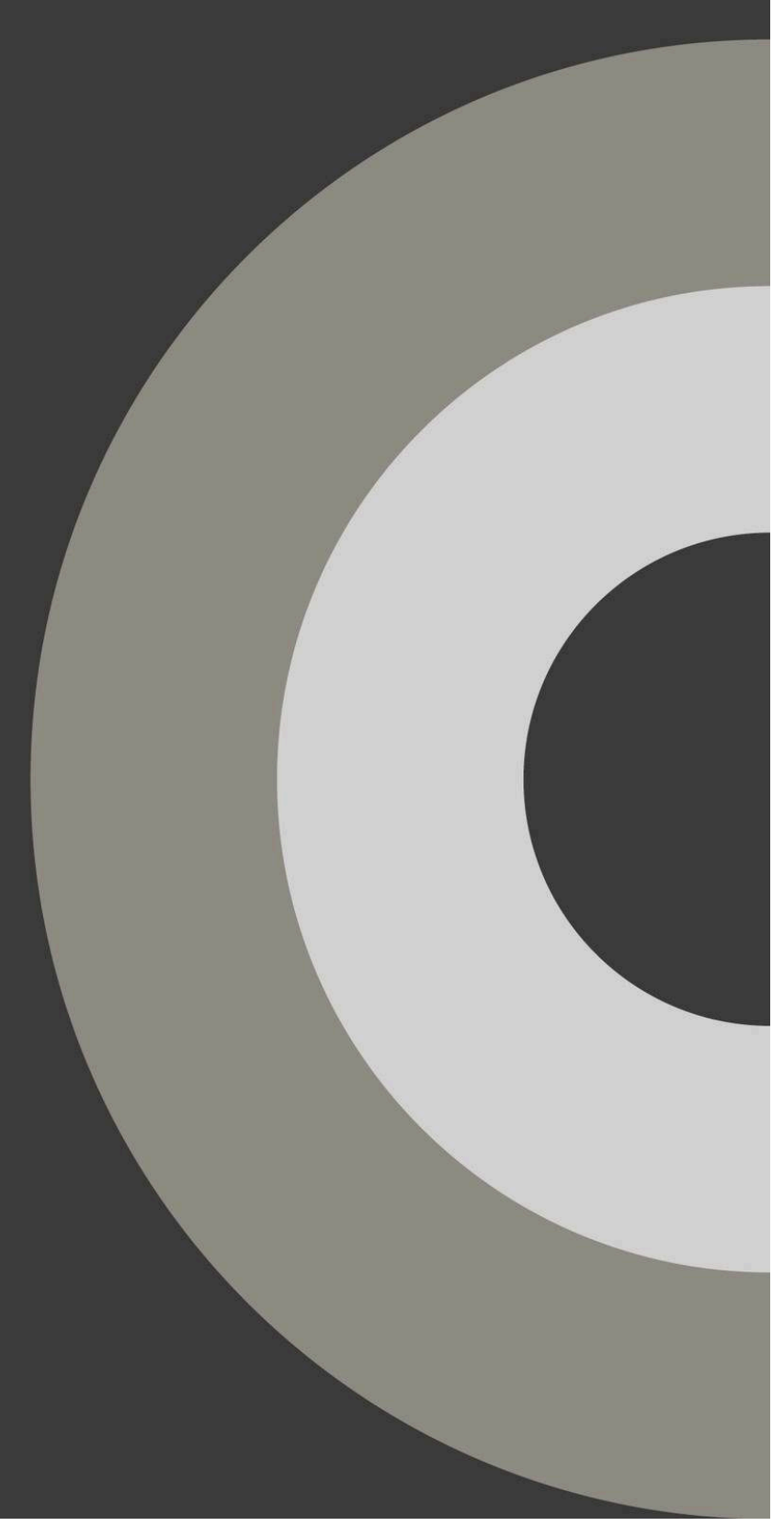




\section{Mondes réels, mondes virtuels}

Jean Soumagne (dir.)

DOI : $10.4000 /$ books.cths. 15840

Éditeur : Éditions du Comité des travaux historiques et scientifiques Année d'édition : 2021

Date de mise en ligne : 17 novembre 2021

Collection : Actes des congrès nationaux des sociétés historiques et scientifiques

EAN électronique : 9782735509294

\section{Sbooks}

http://books.openedition.org

\section{Référence électronique}

SOUMAGNE, Jean (dir.). Mondes réels, mondes virtuels. Nouvelle édition [en ligne]. Paris : Éditions du Comité des travaux historiques et scientifiques, 2021 (généré le 19 novembre 2021). Disponible sur Internet : <http://books.openedition.org/cths/15840>. ISBN : 9782735509294. DOI : https://doi.org/ 10.4000/books.cths. 15840 .

Ce document a été généré automatiquement le 19 novembre 2021.

(C) Éditions du Comité des travaux historiques et scientifiques, 2021 Conditions d'utilisation :

http://www.openedition.org/6540 
La réalité de la planète Terre a fait l'objet d'innombrables spéculations, nourrissant dès l'Antiquité gréco-latine une foule de discours, de descriptions, de récits. Dans le présent recueil, les modes d'appréhension des mondes réels et des mondes virtuels sont à géométrie variable et fournissent un éventail ouvert de ces modes.

Le Congrès national des sociétés historiques et scientifiques rassemble chaque année universitaires, membres de sociétés savantes et jeunes chercheurs. Ce recueil est issu de travaux présentés lors du $144^{\mathrm{e}}$ Congrès sur le thème « Le réel et le virtuel ».

\section{JEAN SOUMAGNE}

Professeur émérite des universités en géographie et aménagement urbain, membre du laboratoire Espaces et sociétés (ESO, UMR 6590, université d'Angers/CNRS) 
SOMMAIRE

Introduction

Jean Soumagne

\section{Représenter le monde}

Du réel aux virtuels, du virtuel aux réels: ce que la cartographie fait au monde Mathilde Joncheray et Delphine Montagne

Cartographier le territoire reflète et transforme notre vision du monde

Les représentations de mondes virtuels : que nous apprennent-ils de notre monde?

La cartographie 2.0 : le virtuel transforme-t-il les cartes?

Dynamique transitionnelle de l'espace hétérotopique : configurer, reconfigurer espaces et contre-espaces

Agnès Besson

Le lieu hétérotopique : émergence et réémergence d'espaces protéiformes

Traverser, demeurer : la transition requiert de nouvelles spatialités interstitielles

L'écriture du regard géographique de Patrick Geddes (1854-1932) : le Collège des Écossais à l'université de Montpellier

Sabine Kraus

Naissance de la géographie humaine et culturelle

Paysage sous-marin et géoarchéologie littorale : le cas de pointe de Porzh Karn en Bretagne (Penmarch, Finistère)

Yves Petit-Berghem et Gaëtan Jolly

Le paysage sous-marin : un concept émergeant à la croisée des disciplines

Analyser le paysage sous-marin : application à la géoarchéologie littorale

Analyse sous-marine et gestion patrimoniale des zones côtières

\section{Imaginer l'aménagement}

Des sources pour l'histoire de l'aménagement du territoire : les archives du tunnel sous la Manche conservées aux Archives nationales

Stéphane Rodriguez-Spolti

Cent mètres linéaires d'archives pour un siècle et demi d'histoire

Les premiers projets (1867-1888)

Quand l'idée de franchir la Manche enflamme l'imagination (1873-1930)

Le virtuel se concrétise : les tunnels de la seconde moitié $\mathrm{du} \mathrm{xx}^{\mathrm{e}}$ siècle

Relier les collines de Fourvière et de la Croix-Rousse à Lyon (1847-1990)

Françoise Bayard

Les auteurs des projets

Quel pont pour quel Lyon?

Emporter la décision

Expliquer les échecs 


\section{Voyage réel, quête virtuelle}

Entre réel et imaginaire, un monde de synthèse : les voyages de Vincent Leblanc, Marseillais Henri Bresc

Vie et voyages de Vincent Leblanc

Les deux rédactions

Le monde virtuel de Vincent Leblanc

Le trésor perdu de l'atoll de Pinaki (archipel des Tuamotu, Polynésie française) vers 1859-1994 au moins...

Véronique Dorbe-Larcade

Une intrigue

Dénouement et interprétation

\section{Du pouvoir fantasmé}

La frontière politique : réalités géopolitiques complexes ou représentations idéologiques partisanes à partir de l'exemple de la vallée du Rhône en Révolution Nicolas Soulas

Une géographie politique rhodanienne très complexe

Des frontières politiques fantasmées?

L'autorité coloniale entre réel et virtuel : moissons, transhumance, interdictions et transgressions en Algérie (1935-1950)

Isabelle Chiavassa

Le virtuel, la norme : maîtrise de l'espace et du temps par l'autorité coloniale

La réalité : un écart entre réel et virtuel

L'État colonial s'adapte

Conclusion

Jean Soumagne 


\section{NOTE DE L'ÉDITEUR}

Les articles de cet ouvrage ont été validés par le comité de lecture des Éditions du Comité des travaux historiques et scientifiques dans le cadre de la publication des actes du $144^{\mathrm{e}}$ Congrès national des sociétés historiques et scientifiques tenu à Marseille en 2019. 


\title{
Introduction
}

\author{
Jean Soumagne
}

1 La réalité de la planète Terre a fait l'objet d'innombrables spéculations, nourrissant dès l'Antiquité gréco-latine une foule de discours, de descriptions, de récits. Certes, les bases d'une perception ordonnée de «ce qui est » et celle d'une description raisonnée du monde ont été posées avec méthode par de grands philosophes; elles furent appliquées avec plus ou moins de bonheur par les historiens, les géographes, les voyageurs dont les relations avaient souvent pour objectifs de dresser un état des lieux, en tentant d'embrasser la totalité de ce qu'ils examinaient. Cet encyclopédisme, classé selon les principes aristotéliciens, a servi de socle aux tentatives multiséculaires de réalisation d'un tableau de la Terre et des hommes jusqu'à l'époque moderne. Cependant, face aux vides béants de la connaissance de certains peuples et de leur environnement, peu ont résisté, parmi les auteurs anciens ou médiévaux, à la tentation d'exploiter des récits oraux de voyageurs et de marchands qui mêlaient le réel et les suppositions. Qui plus est, l'appétit pour le merveilleux, pour le spectaculaire, a entraîné plusieurs auteurs à user d'une veine mythique dans leurs descriptions de la Terre, favorisant par là même la rêverie des lecteurs; c'est ainsi que des terres imaginaires, des peuples inventés aux coutumes exotiques, des animaux fantastiques se sont, dans maintes descriptions du monde, entremêlés aux faits véritablement constatés.

2 Si les récits d'«itinéraires" sont souvent d'un grand intérêt, les relations plus consistantes sur la planète et ses hôtes, sur un continent, voire sur un seul pays renferment souvent, durant le Moyen Âge, un mélange d'éléments "sûrs ", et d'éléments supposés, ou inventés. À l'âge même des "grandes découvertes", les conquérants ont souvent anticipé les hypothétiques réalités futures en faisant miroiter auprès des financeurs des voyages tant l'étendue des territoires à visiter et leurs richesses merveilleuses, que les trajets permettant de relier le port de départ aux lieux convoités, tel le célèbre passage du Nord-Ouest.

3 Avec les débuts du rationalisme, le souci d'une connaissance scientifique, cohérente et classée, et plus encore fondée sur un examen de visu du réel, a pris le pas sur l'imaginaire. Sans doute les récits de voyage mettent-ils encore l'accent sur l'étrangeté des coutumes des peuples, sur les faits saillants de leur histoire, sur les particularités 
extraordinaires de leur environnement, mais la dominante se veut une description exacte, précise du « réel ». Ce qui ressortit, au moins connu ou à l'inexploré, fait l'objet de précautions tant de langage que de représentation graphique de manière à être soigneusement distingué de la réalité constatée. Durant le XVIII ${ }^{\mathrm{e}}$ siècle, les progrès des sciences, en botanique, zoologie, minéralogie s'accompagnent de la publication d'ouvrages encyclopédiques au sein desquels l'anthropologie naissante trouve aussi sa place. Embrasser la totalité planétaire est alors devenu aussi bien un objectif général que l'objet spécifique de grandes expéditions. Naturellement, les publications de " voyages extraordinaires » au sein desquels les réalités peuvent être travesties et les mythes récurrents conservèrent aussi leur place dans l'édition.

$4 \mathrm{Au} \mathrm{XIX}^{\mathrm{e}}$ et au $\mathrm{XX}^{\mathrm{e}}$ siècles, les tendances précédentes s'accentuent, rationalisme et positivisme visent toujours à atteindre l'exhaustivité du réel, la part du légendaire et $\mathrm{du}$ mythique n'ayant qu'une place mineure dans la présentation du monde. Les frontières de la réalité de la Terre et des peuples sont repoussées toujours plus loin, aboutissant à des tableaux méticuleux des différentes facettes du réel. En dehors du regard ethnographique, le mythique, le virtuel se trouvent alors laissés à la sphère littéraire, celle du monde fantasmatique, celle des « voyages extraordinaires » et du pur romanesque où les espaces et les temps s'entremêlent en de multiples projections virtuelles.

Ainsi s'est inscrite peu à peu, et de manière le plus souvent dichotomique, la distinction entre le travail scientifique et l'ouvrage distractif.

Cette opposition frontale a subi de sérieux revers avec l'irruption d'une vision moins radicale offrant une place de choix aux interrogations sur la nature du regard porté sur la planète. Les sciences humaines ont justement souligné les différences de perception du réel d'une civilisation à une autre, d'un individu à l'autre, d'un âge de la vie à un autre et selon les acquis socio-culturels. Les imperfections et les variations des cinq sens, les différences de leur poids respectif ont été pointées. L'intérêt de lectures nuancées sur la perception des espaces et des paysages, mais encore des personnes et des groupes humains, est apparu à la faveur des travaux des anthropologues, des ethnologues, des géographes. Les historiens, habitués de longue date au croisement des sources ont ouvert de nouveaux champs d'investigation en travaillant eux aussi sur les représentations des êtres et des lieux à travers leur histoire.

Ces ouvertures débouchent ainsi sur des travaux confrontant, sans les hiérarchiser nécessairement, le monde à un instant $\mathrm{T}$ et les utopies de cet instant, les réalisations transcrivant dans le réel la transformation du monde et les projets aux degrés de « futurisme » variables. Les utopies du passé ont pu être comparées aux réalités et aux utopies du présent. Les représentations littéraires ou artistiques du monde ont fait l'objet de comparaisons dans l'espace et dans le temps.

On ne peut qu'être frappé par la prudence actuelle, voire le détachement, vis-à-vis de l'exercice macroscopique et microscopique d'examen du «réel», sachant que les frontières de cet examen sont sans cesse reportées plus avant par la progression constante des techniques d'étude. Cette relative modestie, ou cette interrogation face aux aléas des représentations se reflètent dans les divers modes de transposition du « réel» qui laissent souvent une place de choix à l'hypothétique et au virtuel. Sans doute peut-on s'interroger sur l'application d'une sorte de "principe de précaution » dans l'examen du «monde réel». Cette omniprésence de l'incertitude ne risque-t-elle pas d'entraîner une certaine paralysie de la recherche par la remise en cause 
systématisée de faits jusque-là bien établis? Ne peut-on pas, en rejetant des outils taxinomiques éprouvés se priver de grilles d'analyse objectivantes autorisant ensuite d'indispensables comparaisons?

9 Les sciences humaines et sociales se trouvent peut-être à une croisée des chemins ; d'un côté, un élargissement de leurs champs de travail qui ne peut qu'apparaitre profitable ; de l'autre, une remise en cause de l'outillage conceptuel et méthodologique, remise en cause qui est susceptible de stériliser l'approche de certains domaines.

Dans le présent volume, les modes d'appréhension des mondes réels et des mondes virtuels sont à géométrie variable et fournissent un éventail ouvert de ces modes.

11 Plusieurs auteurs se sont intéressés aux modes et aux champs de la représentation du monde : quels regards, sur quels objets, avec quels outils ? La cartographie, la réflexion philosophique sur l'utopie et l'hétérotopie, le projet architectural, les techniques de pointe d'investigation sous-marine sont tour à tour sollicités.

12 Une deuxième thématique porte sur l'imaginaire dans l'aménagement : comment ont été rêvés le franchissement des obstacles naturels et la liaison entre des espaces, ceci à deux échelles, continentale et locale. D'un côté une utopie multiséculaire a réussi, de l'autre un simple projet urbain est resté lettre morte.

13 Dans un troisième temps, c'est le voyage lointain qui est examiné. Dans un premier texte, il s'agit ainsi de faire l'inventaire du réel et de l'imaginaire dans les récits d'un voyageur du XVII ${ }^{e}$ siècle. En revanche, un second article permet de se pencher sur des voyages bien réels, d'« explorateurs » qui ont été attirés dans la recherche d'un trésor mythique ou réel - d'une île polynésienne, l'attraction fabuleuse du trésor insulaire se croisant à celle, mythique, du Pacifique Sud.

14 Enfin, en une dernière partie, la virtualité et la réalité des limites et des territoires sont interrogées dans deux articles d'histoire contemporaine. Le premier se situe dans le champ politique et décrypte, au temps de la Révolution française, les représentations intercommunales des antagonismes politiques, et les confronte aux réalités électorales. Le second interroge l'opposition entre la réalité des limites formulées par l'administration à la transhumance et les transgressions qu'elle supportait face à un semi-nomadisme, fondé par essence sur le jeu entre réel et virtuel.

\section{AUTEUR}

\section{JEAN SOUMAGNE}

Professeur émérite des universités en géographie et aménagement urbain, membre du laboratoire Espaces et sociétés (ESO, UMR 6590, université d'Angers/CNRS) 
Représenter le monde 


\title{
Du réel aux virtuels, du virtuel aux réels : ce que la cartographie fait au monde
}

\author{
Mathilde Joncheray et Delphine Montagne
}

1 Qu'elle ait été créée sur un support physique il y a plusieurs siècles ou qu'elle soit disponible sur un site Internet aujourd'hui, la carte est une représentation graphique et mentale du monde. Ce qui y est figuré est sélectionné, mis à une taille réduite selon une échelle tout en essayant généralement d'être fidèle à la réalité. Néanmoins, du fait des choix de représentations et de sélections, la carte n'est pas exactement le réel. « Miroir des hommes et du monde» (Picouet, 2018), la carte permet de représenter le monde, mais également de transcrire nos représentations du monde.

«La réalisation de chaque carte est une invention, car elle révèle et donne à voir ce qui était caché du monde dans un acte de représentation créatif subjectif et partiel

d'une réalité inaccessible ${ }^{1}$. »

2 Nous imprégnons les cartes de nos représentations, et à leur tour ces représentations nous amènent à transformer le monde qui nous entoure. C'est sur ce paradoxe, cette tension que porte notre discours : entre géographie virtuelle et réelle puis inversement, nous réaliserons une approche croisée. Elle s'appuie sur nos expériences d'enseignantechercheuse et d'ingénieure d'étude-cartographe, qui associent pratique personnelle de cartographie, enseignements proposant réflexions et exercices cartographiques, formation et participation à de la cartographie participative (cartographie 2.0).

Nous choisissons ici d'utiliser trois définitions du virtuel - des virtuels - afin de les mettre en regard du réel, c'est-à-dire de l'espace tangible qui nous entoure. Le premier virtuel dont nous parlerons, c'est la carte. En effet, une carte est la représentation d'un espace, ce n'est pas exactement le réel : une carte est donc une représentation mentale de l'espace. Mais elle a beau être virtuelle, la carte façonne le monde. L'écart entre cartographie du monde réel et cartographie de mondes virtuels est mince : pourquoi la cartographie (virtuelle, donc) de mondes virtuels n'aurait pas elle aussi des conséquences sur le monde réel? Enfin, au xxI siècle, c'est aussi l'ordinateur et Internet qui, dans un nouvel espace virtuel, ont permis l'émergence d'un double spatial 
numérique appelé « géocyberespace » (Bakis, 2007). Cet espace numérique - virtuel contient des représentations du monde réel comme des mondes virtuels. Nous verrons comment ce géocyberespace transforme les cartes et le monde réel.

\section{Cartographier le territoire reflète et transforme notre vision du monde}

4 La carte n'est pas la réalité (même réduite), mais une suite de choix qui permettent de faire passer un message. L'histoire de la cartographie est une suite de compromis entre recherche de la carte la plus «vraie» possible, et pour laquelle les évolutions des techniques vont permettre d'atteindre au plus près cet effet de réel, et projection de ses représentations sur le support cartographique.

5 Nous n'allons pas retracer toute l'histoire de la cartographie, d'autres l'ont bien fait ${ }^{2}$. Nous allons simplement nous attacher à montrer l'alternance historique fondamentale entre la volonté de représenter la réalité spatiale au plus près du réel, et la volonté de traduire au mieux ses propres représentations.

6 La plupart des premières cartes du monde ont une dimension pratique : il s'agit de se repérer. En Mésopotamie (Irak), lieu de l'apparition des premières villes de l'Histoire, de l'Écriture et des premières cartes, a été retrouvée une carte datant d'environ 2500 avant notre ère. Elle représente des montagnes, des rivières, avec une localité au centre (fig. 1a). S'appuyant sur les travaux de leurs prédécesseurs mésopotamiens et égyptiens, les Grecs ont systématisé la recherche cartographique basée sur les mathématiques et la cosmographie : Anaximandre de Milet ( $\mathrm{vI}^{\mathrm{e}}$ siècle av. J.-C.) serait ainsi le premier à proposer une carte du monde connu (fig. 1b) et Ératosthène (II siècle av. J.-C.) le premier à mesurer la circonférence terrestre. C'est enfin Claude Ptolémée (II siècle) qui a fixé l'utilisation du système de coordonnées utilisant la latitude et la longitude.

Fig. 1. - Reproduction des cartes de Ga Sur (A) et d'Anaximandre (B).
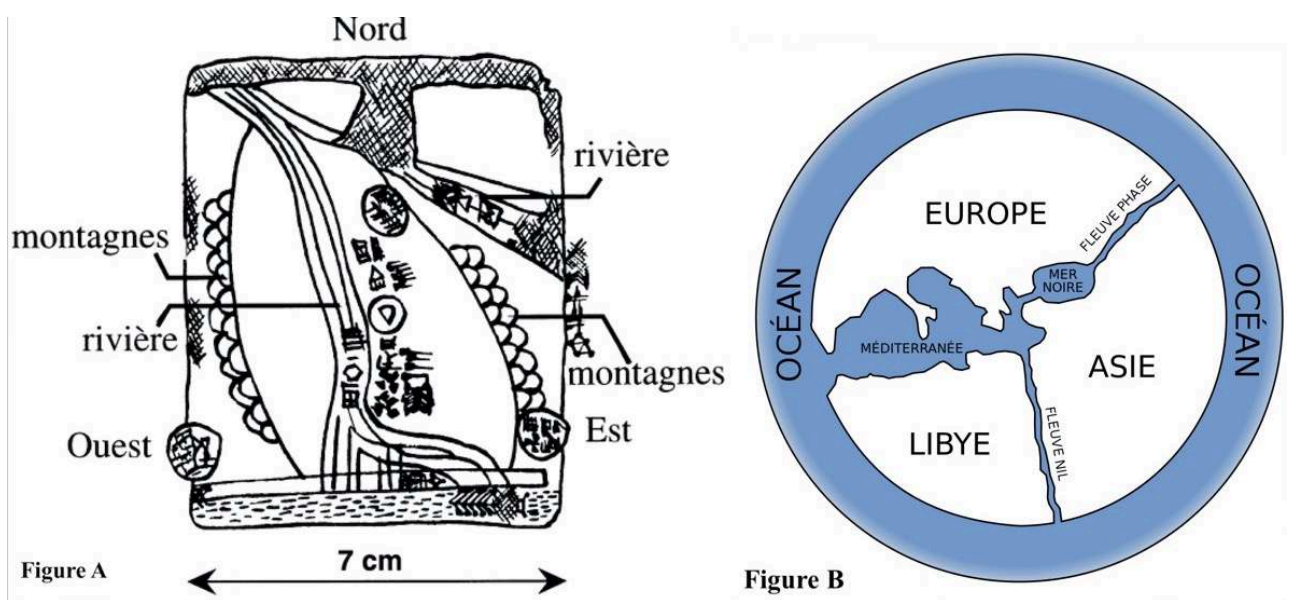

(A) Carlo Denis Wikipedia CC BY-SA 3.0. (B) Bibi Saint-Pol, adaptation de Gwwfps, d'après l'ouvrage de John Mansley Robinson, Domaine public.

7 Les Grecs s'appuient ainsi sur la science pour établir leurs cartes; ils mesurent, arpentent, calculent... À la Renaissance, les Européens se sont appuyés sur ces travaux 
pour bâtir des cartes présentées comme les plus proches du réel, jusqu'à l'établissement de normes donnant aux cartes actuelles, surtout si elles sont éditées par des instituts reconnus (tel l'Institut Géographique National en France), une valeur d'objectivité largement reconnue. Des tests menés dans nos cours montrent que sur une échelle de 0 (pour subjectif) à 5 (pour objectif), les cartes topographiques de l'IGN ont quasiment toujours la note maximale. D'autres expériences de ce type (nous pensons par exemple à celle relatée par Bénédicte Tratnjek ${ }^{3}$ ) confirment ce phénomène. Or les normes actuelles ne sont que la résultante de choix opérés dans la longue durée par des générations de cartographes.

8 Les Grecs ont donc inspiré les Européens par leur "scientificité ». Mais si ce que nous avons retenu d'eux est leur héritage scientifique, leur cartographie n'est pas aussi scientifique qu'il y paraît. Astronomie et astrologie ne sont pas si éloignées, et la représentation d'un monde plat pour les uns (Anaximandre, $\mathrm{v}^{\mathrm{e}}$ siècle av. J.-C.) ou sphérique pour les autres (Ératosthène) est tout autant affaire de représentations (Grataloup, 2011).

9 Les cartes ont ainsi longtemps véhiculé le message que la vérité n'était pas forcément le monde réel. La cartographie arabe médiévale, héritière directe de la cartographie grecque, ainsi que la cartographie européenne à la même époque, ont par exemple concilié ces deux tendances que nous opposons aujourd'hui : recherche de la réalité scientifique et recherche de la réalité théologique. Le Moyen Âge offre ainsi des contrastes saisissants entre des cartes " $\mathrm{T}$ dans l'O " d'un côté, basées sur les représentations bibliques, et les premières cartes marines (portulans) avec havres et lignes des vents, nécessaires à la navigation. Mais ces oppositions ne le sont que pour nous, tant il n'existait pas forcément d'opposition entre monde réel et symbolique.

De la même manière, il n'était pas concevable de laisser les espaces vides en blanc. Les terras incognitas ou les océans étaient peuplés de créatures effrayantes, réelles ou inventées, sans que cela ne fasse baisser la valeur de la carte, bien au contraire. Au-delà de l'aspect purement artistique de cette iconographie, l'ajout de ces dragons et animaux marins fantastiques avait des fonctions didactiques, religieuses, voire économiques. Barbara Muller (2016) montre par exemple à travers l'analyse de la Carta Marina de Olaus Magnus (1539) que les monstres marins servent à prévenir les marins du danger, mais permettent également de dissuader les pêcheurs concurrents des pays voisins d'entrer dans les mers scandinaves.

11 Le primat accordé en Europe à partir des grandes découvertes $\left(\mathrm{xv}^{\mathrm{e}}\right.$ siècle) à la technique et à la véracité scientifique a eu raison de l'imaginaire en matière de cartographie, ce qui oriente nos lectures actuelles et nous pousse à opposer cartes «vraies» ou «fausses». L'avènement de la science cartographique aux xvII ${ }^{\mathrm{e}}$ XVIII ${ }^{e}$ siècles en France a abouti à la mise en cartes de la plupart des territoires nationaux, à travers de grandes opérations de cartographie. L'utilisation de la longitude, mesurée à partir de l'invention du chronomètre (1736), et l'établissement de coordonnées géographiques (mesurées en angles) ou planes (mesurées en unités de surface) permettent de donner un air strictement rigoureux à la cartographie. Mais les projections (puisque c'est de cela qu'il s'agit) ne sont que des projections de nos esprits, et ne permettent que maladroitement de transcrire le géoïde terrestre (donc pas tout à fait sphérique) sur une surface plane. Nous avons tellement intégré l'image de la projection qui s'est imposée au Xvi siècle, celle de Mercator, qu'on oublie souvent qu'elle résulte de systèmes de pensée bien particuliers : adaptée aux voyages en mer à 
partir des côtes européennes, associée à une vision du monde européo-centrée (l'Europe est au centre de la carte et l'hémisphère nord, en haut de la carte, est surreprésenté), elle a figé pour des siècles nos représentations du monde. Désormais contestée dans le milieu des cartographes, nombreux sont ceux qui créent de nouvelles projections et décentrent nos regards (un exemple particulièrement intéressant en est la projection de Spilhaus ${ }^{4}$ ). Mais ces visions alternatives du monde sont encore très peu intégrées dans les représentations du grand public. Un exemple frappant est la façon récurrente avec laquelle les étudiants, lors de leurs premiers cours sur la carte, expriment le fait que les cartes qui présentent une orientation différente sont "à l'envers ", ou le succès des articles sur les projections (souvent accompagnés de titres percutants ${ }^{5}$ ), qui ne sont pourtant pas des plus faciles à comprendre, selon nos étudiants. Or cela fait plus de quarante ans que McArthur (1979) a réalisé son "Universal Corrective Map of the World ${ }^{6}$ ", présentant un planisphère centré sur l'Australie, sud en haut; mais les représentations du monde qui nous décentrent du Monde restent peu normalisées.

12 La toponymie elle-même (les noms de lieux), que l'on pourrait croire « neutre ", révèle la conception européo-centrée du monde qui nous entoure : "Mers du Sud », Amérique, (d'Amerigo Vespucci), l'Orient (c'est-à-dire l'Est... de l'Europe) en sont autant d'exemples durables, malgré de nombreuses «corrections » effectuées ces dernières années (la transformation d'anciens toponymes coloniaux notamment) et des mouvements d'interpénétration culturelle plus complexes; on utilise de plus en plus l'expression «l'empire du Milieu» par exemple. A-t-on pour autant toujours conscience du phénomène parallèle de sino-centrisme que cela révèle, et qu'il existe d'autres cartographies sur la planète?

Nos représentations du monde sont en outre des représentations sédentaires de l'espace. Elles écartent les mers (à l'exception de la projection de Spillhaus centrée sur l'Antarctique, ce qui augmente d'autant plus son impression d'étrangeté), considérées comme négligeables et tracent des frontières, figent les territoires. Or certains territoires ne peuvent être délimités par des traits sur une carte. Sans même évoquer les territoires nomades superposés aux territoires sédentaires, il existe quelques zones de territoires strictement nomades: au Sahara (cartographie des «fuseaux» de $\left.\operatorname{Monod}^{7}\right)$, ou en Océanie par exemple. Dans ce dernier cas, il existe des cartes, encore en usage jusqu'à la Seconde Guerre mondiale, faisant état des itinéraires maritimes de la population, mentionnant vents, houles et vagues ${ }^{8}$.

14 La plus belle des cartes, celle qui parait la plus juste, la plus fidèle au réel, résulte donc de nombreux choix de représentations et de biais de position (projection, choix du centre, de l'échelle, de la toponymie), et ne constitue qu'une proposition de représentation du monde réel. Ces biais méthodologiques sont également présents dans la cartographie des mondes virtuels, à la différence que nous sommes, pour ces derniers, bien moins engoncés dans les normes de cartographies, ce qui laisse place à une plus grande diversité de cartes, et peut peut-être contribuer à modifier nos représentations du monde. 


\section{Les représentations de mondes virtuels : que nous apprennent-ils de notre monde?} vraies dynamiques touristiques existent autour de lieux emblématiques comme Ystad, en Suède, lieu où se trouve le bureau de l'inspecteur Wallander, héros des romans policiers de Henning Mankell (voir Saumon et al, 2015). Le même phénomène est également étudié pour les lieux de tournage de fictions prenant place dans des lieux imaginaires, mais dont les lieux de tournage sont, eux, réels, à l'exemple de ces magazines ou agences de voyages qui proposent la visite des lieux emblématiques de Game of Thrones ${ }^{9}$. Ce qu'on appelle le set-jetting ${ }^{10}$, étudié par T. Joliveau (2015), est une réalité économique, comme le montre l'exemple de Tchernobyl : depuis le succès de la série télévisée Chernobyl (2019), les touristes affluent sur le site de l'accident ${ }^{11}$, tout comme sur celui du tournage en Lituanie, dans une centrale nucléaire désaffectée ${ }^{12}$. Pour surfer sur ce phénomène, la fiction laisse enfin sa trace dans le paysage, donnant à voir ce que cherchent les visiteurs : création du Central Perk à Greenwich Village (New York) pour les nostalgiques de Friends ou d'enseignes Le Mistral à Marseille pour les fans de Plus belle la vie. 
19 L'imaginaire sert ainsi à penser le monde d'aujourd'hui et ses représentations, tout comme il peut servir à penser le monde de demain. À cet égard, le livre d'Alain Musset, De New York à Coruscant (2005) constitue un modèle du genre. Véritable manuel de géographie, il utilise l'exemple de la capitale de la République (puis de l'Empire) du monde de Star Wars pour montrer notamment les avenirs possibles de la croissance urbaine. Plus globalement, les dystopies (surtout urbaines) et la littérature de sciencefiction permettent de construire différents scénarii de prospectives, amenant à penser le monde de demain. La démarche n'est guère différente de celle de la Datar proposant des cartes de prospectives (Datar 2020 et 2040 ayant fait date). Ainsi, la cartographie d'espaces virtuels peut provenir directement d'espaces réels, tout comme les espaces virtuels peuvent aider à penser le réel.

Si la fiction est donc un reflet de notre monde, la carte peut être lue comme un « miroir des hommes et du monde » (Picouet, 2018). Les cartes de nos imaginaires projettent nos représentations, mais aussi nos désirs. Et parfois les anticipent. C'est par exemple le cas de la célèbre carte du Maraudeur d'Harry Potter (Le prisonnier d'Azkaban, 1998), qui confère au héros un avantage conséquent en lui permettant de suivre à la trace en temps réel ses camarades dans le château de Poudlard. La réalité a rejoint la fiction en faisant d'un objet magique en 1998 un objet commun en 2020. L'utilisation des « minimaps » dans les jeux vidéo a également anticipé l'arrivée sur le marché des GPS (Global Positioning System) : les premiers GPS grand public datent de 2004, avec l'arrivée du TomTom GO; or un jeu comme Metal Gear Solid, qui contient une mini-map, date de 1998. On assiste d'ailleurs à un renversement de situation avec des titres de jeu vidéo qui refusent désormais les "mini-maps", trop invasives et freinant l'immersion du joueur en le guidant de manière trop directive. Or qui ne s'est pas fait la remarque, en se laissant guider par un GPS, qu'il ne saurait pas retrouver son chemin sans lui ? L'arrivée des outils numériques a donc des impacts de plus en plus grands sur les espaces et nos pratiques de l'espace, qu'ils soient réels ou virtuels.

\section{La cartographie 2.0 : le virtuel transforme-t-il les cartes?}

21 L'informatique a été une révolution pour la cartographie: l'ordinateur offre une imposante puissance de calcul, permet des modélisations poussées, et via Internet offre une plus grande accessibilité au contenu cartographique (globes virtuels, SIG, statistiques). Google Maps met en avant les annonceurs ou les commerces : afin d'être visible en premier, il faut payer pour être à une petite échelle de la carte. Les cartes Michelin font apparaître seulement les lieux où les voitures circulent. Quant à OpenStreetMap (OSM), la cartographie libre et collaborative, même si elle reste de qualité inégale selon les lieux du monde, dans les lieux fréquentés par des contributeurs réguliers elle atteint une grande qualité. L'IGN propose également une carte numérique issue de ses cartes topographiques sur le Géoportail, mais qui reste limitée à la France. Ces quatre exemples de cartes présentent un gradient allant d'un monde commercial à un monde encyclopédique, mais portant chacune des blancs, donc en décalage vis-à-vis du réel.

22 L'utilisation des globes virtuels, notamment de Google Earth, le premier à avoir été mis en service en 2005, a changé notre représentation de la surface terrestre. Ce dernier a facilité l'appréhension du changement d'échelle grâce à la rapidité et la précision du 
« zoom googelien », devenant un standard visuel mondial et entraînant un nouveau rapport à l'espace (Lussault 2007). Glissée dans notre poche au sein de notre téléphone portable (fig. 2), la carte est omniprésente dans de nombreuses applications afin de faciliter l'accès aux services ou proposer des résultats de recherches adaptés à chaque zone géographique (Joliveau et al, 2013). Par les liens avec d'autres applications, la carte devient personnalisée grâce aux publicités finement ciblées en fonction de nos modes de vie et s'adapte à nos modes de déplacement (à pied, à vélo, en voiture, à cheval...). Première grande rupture avec la carte papier, l'utilisateur y apparaît en son centre avec une flèche d'orientation pour faciliter sa propre localisation dans l'environnement. Deuxième rupture, le nord n'est plus forcément en haut même si notre première partie a montré que cela n'avait pas toujours été la norme.

Fig. 2. - Interface d'OSMAnd. La carte tourne autour de l'utilisateur et l'on peut zoomer et dézoomer à volonté.

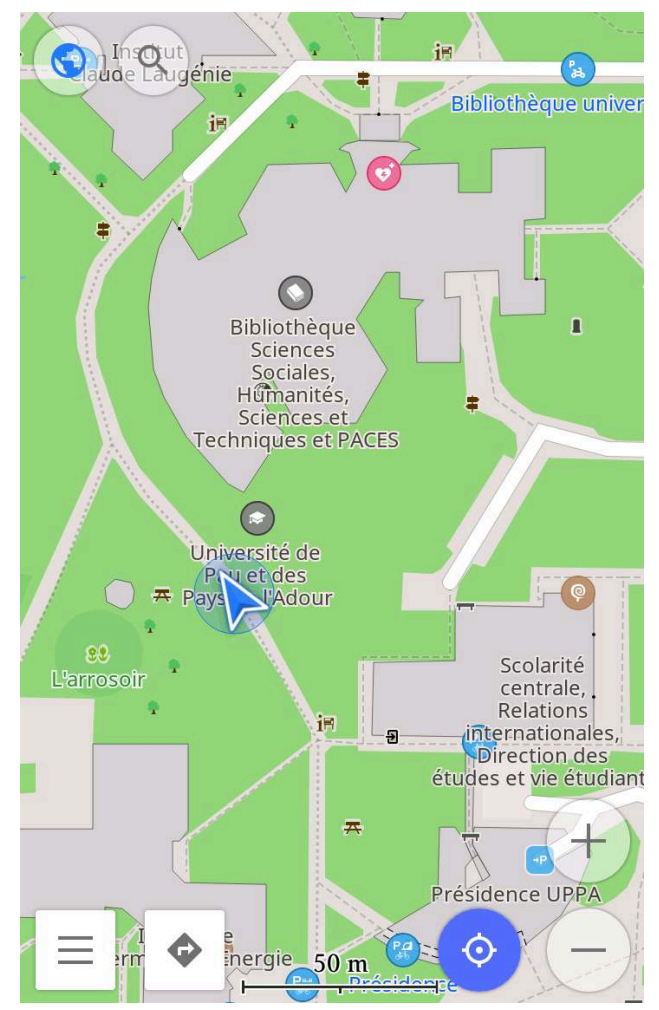

Données @ les contributeurs OpenStreetMap

Troisième rupture, l'apparition de multiples petits mondes virtuels personnalisés : les géographes parlent d'ego-cartographie (Noucher, 2017). Alors même que les connaissances cartographiques se diffusent, que le modèle « mercatorien » basé sur un planisphère européo-centré est remis en cause, nos principaux outils nous placent nous, individus, au centre du monde... et également en situation de toute-puissance au sein du « géocyberespace » mondial (Bakis, 2007).

En effet, qu'elle soit numérique ou papier, la carte n'est plus simplement une carte : c'est une énorme base de données (6,8 milliards de nœuds pour OSM début 2021), ce qui permet de faire des cartes par sujet en sélectionnant uniquement certaines données ou au contraire de proposer plusieurs choix de cartes de manière peu coûteuse, comme 
des cartes en plusieurs langues étrangères ou régionales que l'on peut sélectionner d'un clic (fig. 3).

Fig. 3. - Fond de carte OpenStreetMap faisant apparaître les toponymes en béarnais.

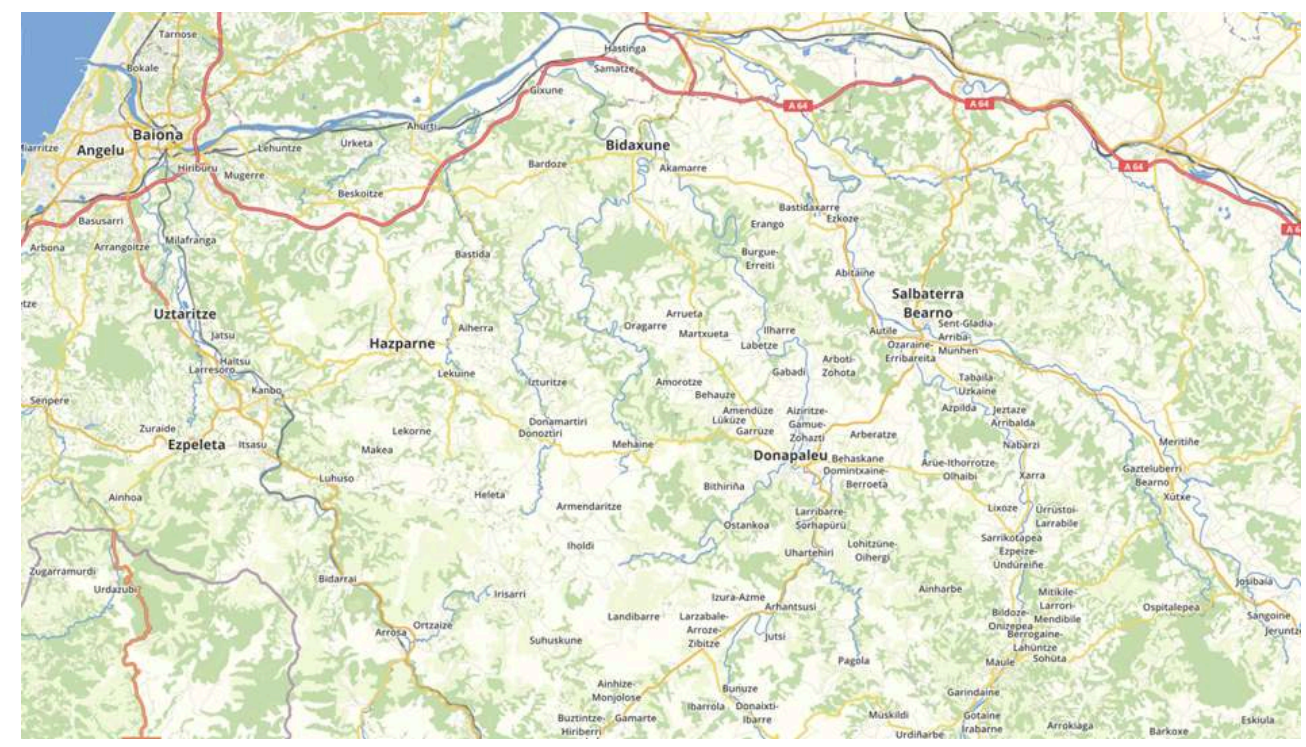

Données @ les contributeurs OpenStreetMap

Créées facilement et sur mesure par tout un chacun, elles sont destinées à proposer un choix au public qu'elles cherchent à toucher. Elles sont autant de représentations particulières du monde offertes à l'utilisateur. Ce sont "les petites cartes du web ", terme proposé en opposition aux cartes institutionnelles (Noucher, 2017). Il n'y a donc plus forcément un seul choix à effectuer... y compris sur le style de la carte, personnalisable à l'infini (carte façon "gaulois", «métal», nocturne), ode à la créativité que l'on peut d'ailleurs imprimer en 3D pour les adapter à la réalité vécue par différents publics (scolaire, malvoyant).

La technique permet aussi de participer simplement à la co-construction des cartes. OpenStreetMap est une carte qui, de par sa nature collaborative, est aisée à modifier, que ce soit grâce à des enquêtes de terrain ou des images satellites; le cartographe amateur peut faire apparaître rapidement le réel, par exemple un camp de réfugiés cartographié à la faveur d'un mapathon humanitaire ${ }^{13}$ (fig. 4). 
Fig. 4. - Le camp de Kakuma (Kenya) avant (à gauche) et après (à droite) la cartographie réalisée lors d'un mapathon sur OpenStreetMap.

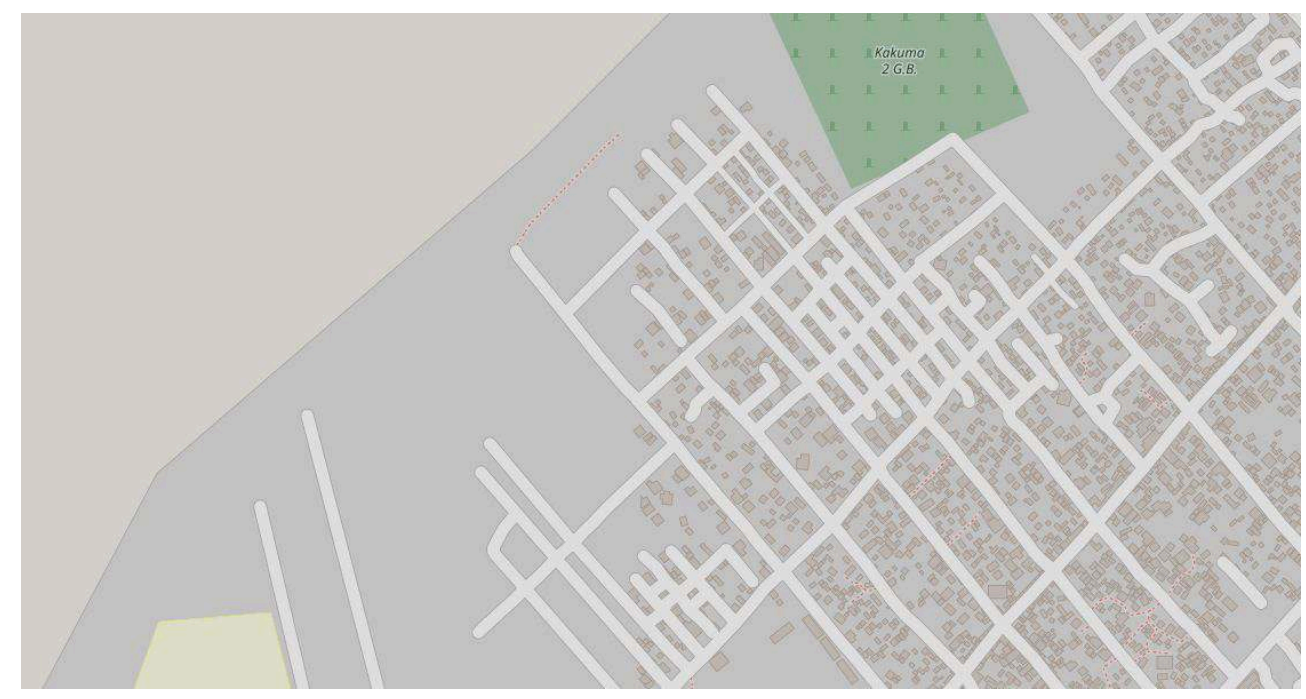

Données @ les contributeurs OpenStreetMap

Cet outil cartographique permet de faciliter la coordination des acteurs locaux et internationaux qui travaillent et éditent tous sur la même carte. La cartographie de populations ou de communautés a aussi potentiellement des conséquences politiques dans l'accès aux ressources ou l'aménagement du territoire, et permet de rendre moins vulnérables les personnes dont la réalité de l'existence n'existe que sur ces cartes.

Pour le contributeur qui participe de son fauteuil, grâce aux images satellites, à cette cartographie mondiale, bâtiment par bâtiment, c'est aussi se rendre compte de la réalité éloignée des déplacements de population, des conditions de terrain et des modes de vie. Pour le contributeur qui cartographie son quartier, seul ou à travers des " cartoparties ${ }^{14}$ ", c'est apprendre à voir son environnement autrement, en redécouvrir les détails, son organisation, des changements inaperçus par la force de l'habitude... La carte s'ouvre à un public diversifié (femme, enfant, handicapé, personne âgée), chacun étant capable d'apporter sa propre vision, son propre vécu et ses préférences, appelés avec humour les TOC (Troubles Obsessionnels de Cartographie; Duféal et Noucher, 2017). Le virtuel devient ainsi un outil de médiation avec tous les publics pour s'approprier la réalité de son environnement.

Ces cartes virtuelles ont enfin des conséquences très réelles. Être présent sur la toile, c'est y laisser des traces virtuelles de nos recherches, de nos pensées et bien entendu de nos déplacements (Mericskay et al, 2018). L'un des exemples les plus marquants est lié à STRAVA, une application qui permet de partager ses traces GPS de courses à pied. La plateforme permet une dynamique de groupe motivant ses pratiquants et a été, à ce titre, promue par les instances militaires. Les militaires courent autour de leur caserne, dans leur propre pays... mais aussi à l'étranger, rendant réel au mieux la localisation des casernes ou, plus délicat, une intervention non officielle ${ }^{15}$.

Autre exemple, afin de fluidifier le trafic, l'application de déplacements routiers Waze, propose des itinéraires alternatifs dans de petits villages qui voient leur fréquentation augmenter fortement, conduisant les maires à des stratégies très fines pour combattre cet ennemi virtuel ${ }^{16}$, amenant, au final, à changer le réel pour impacter la géographie virtuelle. 


\section{BIBLIOGRAPHIE}

BAKIS Henry, 2007, « Le “géocyberespace” revisité », Netcom, n² 21-3/4, p. 285-296.

BOURON Jean-Benoît, 2010, « Cartographier l'imaginaire : un exercice géographique », Bulletin du comité français de cartographie, $\mathrm{n}^{\circ} 205, \mathrm{p} .11-24$.

CASSAR Jean-Philippe, 2018, « Avant-propos », dans Picouet Patrick (dir.), La carte invente le monde, Villeneuve d'Ascq, Presses Universitaires du Septentrion, 206 p.

DUFÉAL Marina, NOUCHER Matthieu, 2017, « Des TIC au TOC. Contribuer à OpenStreetMap : entre commun numérique et utopie cartographique », Netcom, $\mathrm{n}^{\circ} 31-1 / 2, \mathrm{p} .77-98$.

GRATALOUP Christian, 2011, « Représenter le monde », La documentation photographique, 64 p.

JOLIVEAU Thierry, NOUCHER Matthieu, ROCHE Stéphane, 2013, « La cartographie 2.0, vers une approche critique d'un nouveau régime cartographique ", L'Information géographique, vol. 77, $\mathrm{n}^{\circ} 4$, p. 29-46.

JOLIVEAU Thierry, 2015, « Connecter espaces réels et espaces imaginaires grâce aux techniques géonumériques », https://hal.archives-ouvertes.fr/hal-01419952. 
L'HER Gwendoline, SERVIÈRES Myriam, SIRET Daniel, 2018, « La Cartopartie, une nouvelle forme de balade urbaine déployée par les villes », Les Cahiers de la recherche architecturale urbaine et paysagère, éd. numérique, 3 | 2018,

[URL : http://journals.openedition.org/craup/1003]

LUSSAULT Michel, 2007, L'Homme spatial. La construction sociale de l'espace humain, Paris, Le Seuil, $400 \mathrm{p}$.

MERICSKAY Boris, NOUCHER Matthieu, ROCHE Stéphane, 2018, « Usages des traces numériques en géographie : potentiels heuristiques et enjeux de recherche ", L'Information géographique, Armand Colin, 2018(2), p. 39-61.

MULLER Barbara, 2016, « La Carta Marina : les monstres marins dans l'imaginaire cartographique de la Renaissance », Carto, $n^{\circ} 34$, p. 66-71.

MUSSET Alain, 2005, De New York à Coruscant, Essai de géofiction, Paris, PUF, 190 p.

NOUCHER Matthieu, 2017, Les petites cartes du web. Approche critique des nouvelles fabriques cartographiques, Paris, Éditions Rue d'Ulm/Presses de l'École normale supérieure, 65 p. PICOUET Patrick (dir.), 2018, La carte invente le monde, Villeneuve d'Ascq Presses Universitaires du Septentrion, $206 \mathrm{p}$.

ROSEMBERG Muriel, TROIN Florence, 2017, « Cartographie du Marseille d'un héros de roman policier (Total Khéops de J.-C. Izzo) », Mappemonde, $\mathrm{n}^{\circ} 121,22$ p.

SAUMON Gabrielle, GUYOT Sylvain, MIGOZZI Jacques, 2015, « Du roman policier au territoire touristique. Ystad, Stockholm : enquête sur les phénomènes Wallander et Millénium », Mappemonde, $\mathrm{n}^{\circ} 117,23 \mathrm{p}$.

\section{NOTES}

1. J.-P. Cassar, 2018, Avant-propos, dans Picouet (2018).

2. Signalons ici par exemple les très pédagogiques Représenter le monde de Christian Grataloup (2011) ou l'exposition en ligne de la Bibliothèque Nationale de France: (http://expositions.bnf.fr/cartes/expo/salle1/08.htm).

3. B. Tratnjek, 2016, «Dessine-moi la géographie !»: https:// aggiornamento.hypotheses.org/3279

4. Quand les océans sont au centre du monde, carte de Spilhaus: https:// www.paperblog.fr/8503211/quand-les-oceans-sont-au-centre-du-monde-carte-despilhaus/

5. Un exemple parmi d'autres: «L'impérialisme par la cartographie...»: https:// blogs.mediapart.fr/pablita-faust/blog/190712/limperialisme-par-la-cartographie-avecles-cartes

6. Carte du monde universelle, corrigée par McArthur: http://mapdesign.icaci.org/ 2014/02/mapcarte-38365-mcarthurs-universal-corrective-map-of-the-world-stuartmcarthur-1979/

7. Exemple de carte sur Géoconfluences : http://geoconfluences.ens-lyon.fr/actualites/ eclairage/un-sahara-des-sahara-s

8. Voir des exemples dans Grataloup, 2011, p. 37. 
9. Par exemple https://www.voyagespirates.fr/revue-voyage/21-lieux-de-tournage-degame-of-thrones-que-vous-pouvez-visiter-1_21082 ou https://www.vogue.fr/lifestyle/ voyages/story/voyage-destination-beau-lieu-spectaculaire-tournage-game-of-thronesserie/62.

10. Croisement de jet-set et de setting : cadre, décors, mise en scène.

11. L'article de l'Express: https://fr.express.live/le-tourisme-a-tchernobyl-est-enplein-essor/

12. L'article de France Info: https://www.francetvinfo.fr/culture/series/la-seriechernobyl-dope-le-tourisme-sur-les-lieux-de-tournage-en-lituanie_3582153.html

13. Un mapathon humanitaire est un ensemble de volontaires qui vont cartographier en même temps une même région sur OpenStreetMap pour répondre aux besoins d'acteurs humanitaires.

14. « Une cartopartie est une balade urbaine qui vise à arpenter une zone géographique à plusieurs, afin de produire des données géo-référencées " (l'Her et al., 2018).

15. Voir par exemple à ce sujet l'article du journal Le Monde du 30/01/2018: «L'armée française met ses troupes en garde contre l'application de jogging Strava. »

16. Un exemple parmi d'autres dans Capital le 09/08/2019: «Le grand ras-le-bol des petites villes envahies à cause de Waze » https://www.capital.fr/auto/le-grand-ras-lebol-des-petites-villes-envahies-a-cause-de-waze-1347094

\section{RÉSUMÉS}

Du fait de la diffusion simple d'outils de cartographie, la pratique cartographique s'est beaucoup répandue ces dernières années, dont une partie sur la toile. De plus en plus d'analyses s'interrogent sur les transformations sociales, sociétales, voire psychologiques que créent ces nouvelles pratiques reposant sur des espaces virtuels, nourrissant identités spatiales et prescience de l'espace. Ces expériences sont imprégnées de références historiques et fictionnelles de cartes qui (ré)inventent le territoire et proposent des utopies permettant de penser le monde tel qu'il est ou tel qu'il devrait être. Inversement, ces pratiques alimentent et renforcent le géocyberespace mondial. Entre cartographie et espaces réels et virtuels puis inversement, nous proposons une approche croisée issue des expériences d'enseignement, de cartographie et d'engagement dans le domaine de l'information géographique participative.

\section{AUTEURS}

\section{MATHILDE JONCHERAY}

Maîtresse de conférences en géographie, UMR LISST, Université Toulouse - Jean-Jaurès

\section{DELPHINE MONTAGNE}

Ingénieure d'études en sciences de l'information géographique, Université de Pau et des Pays de l'Adour, E2S UPPA, CNRS, TREE, Pau, France 


\title{
Dynamique transitionnelle de l'espace hétérotopique : configurer, reconfigurer espaces et contre- espaces
}

\author{
Agnès Besson
}

1 Alors que l'espace se donne désormais dans une incommensurable plasticité démultipliant à l'infini ses représentations entre réalité et virtualité, la notion foucaldienne d'hétérotopie semble offrir une mise en perspective des nouveaux territoires de la spatialité. Michel Foucault rappelle d'emblée, dès l'initium de sa conférence de 1967 donnée devant le Cercle d'études architecturales, la prééminence contemporaine des problématiques liées à l'espace et la nature réticulaire de celui-ci :

\footnotetext{
«L'époque actuelle serait peut-être plutôt l'époque de l'espace [...] Nous sommes à l'époque du simultané, nous sommes à l'époque de la juxtaposition, à l'époque du proche et du lointain, du côte à côte, du dispersé. Nous sommes à un moment où le monde s'éprouve, je crois, moins comme une grande vie qui se développerait à travers le temps que comme un réseau qui relie des points et qui entrecroise son écheveau ${ }^{1}$.»
}

2 Foucault pressent ce qui va s'amplifier considérablement tout au long des décennies qui suivront la rédaction de ce texte, à savoir une obsession de l'espace qui voit celui-ci à la fois s'élargir à la mesure de sa globalisation et se contracter à travers son quadrillage territorial.

Dès lors où peuvent se loger les aspirations utopiques ? À quelle spatialité peut aspirer le désir et vers quels territoires peut se tourner la quête des possibles? En effet, cette spatialisation obsédante de toute existence, sa circulation incessante tout autant que sa géolocalisation, détermine la nature de chaque expérience aux prises désormais avec la question de l'habiter dans un monde mouvant.

4 Dans son ouvrage L'Âge des transitions, le philosophe Pascal Chabot relève la récurrence du terme de transition et l'emploi polysémique de ce vocable qui a fini par s'imposer comme le mot à travers lequel notre «époque désorientée ${ }^{2}$ » se reconnaît. Dressant un 
inventaire des expériences et de leurs contextes d'émergence par lesquels le concept de transition se voit mobilisé, il observe comment celles-ci se situent dans « les bordures du système ${ }^{3}$ " et combien ces lisières sont intimement liées au vécu transitionnel des individus. La question des transitions qui traverse notre contemporanéité redistribue notre rapport au réel et sollicite nos capacités imaginatives. Notre époque serait transitionnelle dans la mesure où la transition serait un processus de création continuée, qui lierait, et c'est là l'hypothèse de Pascal Chabot, toutes les activités humaines, la transition écologique allant de pair fondamentalement, voire consubstantiellement, avec la transition démocratique et ayant des répercussions en termes de transition identitaire, l'individu étant toujours appelé à plus de mutations, plus de reconversions, plus de mobilité sur les terrains professionnels comme psychoaffectifs.

Les transitions trouvent alors à s'incarner à travers des lieux hétérotopiques qui sont au plus près de projets disant la nécessité d'une réappropriation des conditions d'existence à travers les ressorts d'un nouvel imaginaire. Ces lieux viennent exprimer une aspiration croissante à la relocalisation de l'expérience, à son ancrage territorial dans des espaces concrets, à la mesure d'un désir de changement dont l'individu puisse évaluer le pouvoir. Il ne s'agit plus tant de projeter son désir dans une dimension de pure virtualité utopique que d'inscrire dans le réel les marques d'une virtualité efficiente. Toutefois, au-delà de la nature nécessairement protéiforme des projets hétérotopiques, la question de leur caractère subversif et du maintien de celui-ci face au pouvoir institué reste ouverte.

\section{Le lieu hétérotopique : émergence et réémergence d'espaces protéiformes}

6 La manière dont Michel Foucault organise sa conceptualisation du néologisme d'hétérotopie est en soi révélatrice de ce qui constitue la notion, à savoir non pas une conceptualité fermée et rigoureuse, mais une approche libre, voire par endroits incertaine, convoquant des expériences concrètes et multiples. Nous pourrions d'ailleurs peut-être observer que si le concept d'hétérotopie a connu une postérité non négligeable dans divers champs des sciences sociales et auprès des urbanistes et architectes, il ne paraît pas avoir acquis une notoriété philosophique conséquente ${ }^{4}$. Le texte de la conférence de 1967 semble offrir une réflexion propre à solliciter l'intuition plus que le raisonnement stricto sensu et le déficit de formalisation conceptuelle dont il témoigne pourrait le rendre insuffisant. Pourtant il apparait au contraire rétrospectivement que la nature peu conventionnelle du texte lui offre une force eu égard aux expérimentations qu'il permet de penser. François Boullant dans une conférence consacrée à la question de l'espace chez Michel Foucault ${ }^{5}$ relève ainsi "l'indiscutable pouvoir de séduction» du propos, mais aussi «l'étrange état d'insatisfaction dans lequel il laisse le lecteur ». Le texte foucaldien, « précurseur » ditil, recèle une réelle ambiguïté à travers sa " prose tout à la fois rigoureuse et rêveuse, méthodique et erratique ». Il paraît par conséquent témoigner à travers sa forme incertaine d'une construction en acte, en cela très proche de son propos, visant l'idée d'un processus ouvert.

7 Dans l'ordre de ce processus ouvert, ce qui est notamment frappant, c'est que Foucault semble avoir, dès 1967, acquis la prescience de la nature réticulaire de notre rapport 
contemporain à l'espace. Cette intuition du réseau et de la circulation par laquelle notre manière d'occuper l'espace se joue désormais est étayée dans sa construction du concept d'hétérotopie par une contextualisation historique. Celle-ci vise à démontrer que l'espace dans l'expérience occidentale a une histoire et que l'obsession de l'espace a émergé en supplantant l'obsession du temps qui hanta notamment le XIX ${ }^{e}$ siècle ${ }^{6}$. Quels sont dès lors les éléments de cette histoire occidentale de l'espace ? Foucault part de l'espace médiéval comme espace de localisation. Au Moyen Âge, l'espace était :

«Un ensemble hiérarchisé de lieux : lieux sacrés et lieux profanes, lieux protégés et lieux au contraire ouverts et sans défense, lieux urbains et lieux campagnards (voilà pour la vie réelle des hommes); pour la théorie cosmologique, il y avait les lieux supra-célestes opposés au lieu céleste; et le lieu céleste à son tour s'opposait au lieu terrestre ${ }^{7}$."

8 Cet espace médiéval de localisation s'est ouvert avec Galilée à un espace infini dans lequel il s'est dissous. C'est alors la découverte de l'étendue et de sa puissance qui sollicite l'imaginaire occidental jusqu'au vertige. Mais l'étendue elle-même se voit supplantée et désormais, c'est l'emplacement qui nous préoccupe et mobilise d'incessants efforts technologiques. Michel Foucault inventorie toutes les questions liées aux techniques contemporaines de stockage, d'archivage, de classement :

«Stockage de l'information ou des résultats partiels d'un calcul dans la mémoire d'une machine, circulation d'éléments discrets, à sortie aléatoire (comme tout simplement les automobiles ou après tout les sons sur une ligne téléphonique), repérage d'éléments, marqués ou codés ${ }^{8}$.

9 L'espace contemporain s'impose sous la forme de relations d'emplacements. Là encore, Foucault parvient à anticiper ce qui deviendra l'obsession des premières décennies du $\mathrm{xxI}^{\mathrm{e}}$ siècle, et on voit se dessiner en germe les problématiques liées aux smart cities et autres villes intelligentes pour lesquelles l'accès à l'espace urbain et son occupation passent par une gestion des flux et des circulations.

10 Foucault insiste en outre sur la nécessité d'analyser les espaces du dehors. À ce titre, il fait référence à la poétique bachelardienne et à l'approche phénoménologique d'un espace chargé d'affects, de rêveries, hanté par nos fantasmes. Il s'agit là d'un espace du dedans au sens où s'y projette notre imaginaire et ce sont les métaphores organiques qui parviennent le mieux à en signifier la plasticité. L'espace bachelardien est celui de la puissance onirique, de la force de la rêverie qui reconfigure les lieux, réinvente la topologie. On voit bien ici que c'est l'intime qui résonne puisque l'immensité cosmique elle-même est le prolongement de cette intimité.

11 Le propos de Foucault quant à lui est d'une autre nature puisqu'il s'agit de penser un certain type d'espace du dehors, un certain emplacement, comme lieu de suspension, de neutralisation ou d'inversion des représentations communes de l'espace. Il s'agit par conséquent de penser des projections du corps social et les lieux du dehors peuvent être eux aussi des projections de l'imaginaire. À ce titre, deux grands types d'espaces du dehors se voient convoqués, l'utopie et l'hétérotopie, comme doubles inversés, le miroir lui-même étant pensé par Foucault comme hétérotopique. Le miroir fonctionne en effet comme une hétérotopie en ce sens qu'il rend, nous dit Foucault :

«Cette place que j'occupe au moment où je me regarde dans la glace, à la fois absolument réelle, en liaison avec tout l'espace qui l'entoure, et absolument irréelle puisqu'elle est obligée, pour être perçue, de passer par ce point virtuel qui est làbas. ${ }^{9}$ " 
12 De ce lien subtil qui noue le réel et le virtuel dans le travail spéculaire complexe du miroitement, l'utopie quant à elle est dépourvue. L'utopie est dégagée de tout ancrage dans le réel, elle est cet emplacement sans lieu réel, pour reprendre les termes de Michel Foucault. Les emplacements utopiques entretiennent avec 1'espace réel de la société :

«Un rapport général d'analogie directe ou inversée. C'est la société elle-même perfectionnée ou c'est l'envers de la société, mais [...] ces utopies sont des espaces qui sont fondamentalement essentiellement irréels ${ }^{10}$. "

Se donne à lire dans l'utopie une logique radicale d'inversion de la norme et l'on pourrait s'attarder ici sur la fascination contemporaine, notamment adolescente, pour ce qu'il est convenu d'appeler les dystopies, récits fictionnels contre-utopiques ou antiutopiques, décrivant des utopies qui auraient mal tourné et qui offriraient à la société un miroir déformant ${ }^{11}$. Mais cette logique utopique tout autant que dystopique n'a aucun ancrage territorial effectif, l'utopie est donc de nature subversive comme production pure de l'imaginaire, virtualité absolue.

L'hétérotopie au contraire oppose sa nature réelle, sa territorialité, et Foucault décèle dans l'espace hétérotopique une sorte d'invariant culturel. L'universalité des hétérotopies se révèle probablement dans toute culture, celle-ci forgeant des lieux réels qui s'inscrivent dans l'institution même de la société comme contre-emplacements. Advient dès lors dans le discours foucaldien une définition de l'hétérotopie comme utopie effectivement réalisée, mais qu'il précise par le travail que celle-ci opère au cœur de la culture sur les emplacements réels existants, qui se voient ainsi à la fois "représentés, contestés et inversés ${ }^{12}$ ». Les lieux hétérotopiques sont alors par nature des lieux hors de tous les lieux, bien qu'ils soient effectivement localisables.

Affinant sa typologie des lieux hétérotopiques (allant jusqu'à produire le néologisme d'hétérotopologie), Foucault est amené à discerner deux contextes de production. Il y a en effet traditionnellement dans les sociétés primitives des hétérotopies de crise visant à prendre en charge des individus momentanément isolés de la société, des situations critiques transitoires : l'adolescence, les menstruations, l'accouchement, ou bien encore le voyage de noces, propre à isoler l'acte de défloration de la jeune fille. Ces hétérotopies, apparentées aux rites de passage, s'absentent dorénavant du paysage de la modernité ou y subsistent de manière marginale. S'y substituent des hétérotopies de déviation, par lesquelles on assigne à résidence les individus dont le comportement est déviant par rapport à la norme. Les maisons de repos, les cliniques psychiatriques, les prisons mais aussi les maisons de retraite sont des lieux incarnant ce type d'hétérotopie. Et à ce titre, les maisons de retraite sont, précise Foucault, à la limite de l'hétérotopie de crise et de l'hétérotopie de déviation, la vieillesse relevant de la crise prise en charge dans les sociétés primitives, mais aussi de la déviation dans une société où le loisir est la norme et où l'inactivité renvoie à l'inutilité sociale. Le cimetière quant à lui décrirait un contre-emplacement hétérotopique pérenne dont les formes évoluent, mais qui serait une sorte d'hétérotopie incontournable. Il est bien un lieu hautement hétérotopique, puisqu'il commence, précise Foucault, avec :

«Cette étrange hétérochronie qu'est, pour un individu, la perte de la vie, et cette quasi-éternité où il ne cesse pas de se dissoudre et de s'effacer ${ }^{13}$. "

$16 \mathrm{Au} \mathrm{XIX}{ }^{\mathrm{e}}$ siècle, le cimetière est délocalisé vers les faubourgs et se voit renvoyé à la périphérie de la ville. A contrario, le jardin est une hétérotopie bienfaisante, « universalisante ${ }^{14}$ » dévolue à l'agrément. Le musée et la bibliothèque sont quant à eux 
des hétérotopies propres à la culture occidentale du XIX ${ }^{e}$ siècle et liées à l'obsession conservatrice, à l'archive. environnant, l'espace ordinaire, comme illusion dénonçant l'illusion du lieu réel, et là serait leur fonction contestataire, ou comme lieux de compensation venant produire un modèle de perfection par leur agencement même, comme dans le cas des colonies puritaines que les Anglais ont fondées en Amérique sur la côte Est ou celui des colonies de jésuites fondées en Amérique du Sud, notamment au Paraguay? In fine, Foucault évoque le bateau, l'embarcation, comme lieu passager, transitoire, et lieu permettant le passage, la traversée, le transit. En cela, l'embarcation, à l'instar de la nef médiévale, nef des fous décrite au début de l'Histoire de la folie, ou le navire, est en soi la figuration parfaite de l'hétérotopie, comme lieu de passage, de transport et réserve d'imagination.

\section{Traverser, demeurer : la transition requiert de nouvelles spatialités interstitielles}

21 L'hétérotopie est en effet de nature transitionnelle et permet de penser les lieux de transit comme lieux effectifs. En cela, le lieu hétérotopique se différencie du Non-Lieu, tel qu'a pu le définir l'anthropologue Marc Augé. Ce dernier, dans l'ouvrage qu'il leur consacre ${ }^{17}$, envisage les Non-Lieux comme des espaces de passage impropres à l'habiter, voire désaffectés. Se repère dans les territoires urbains de la modernité une extension sans précédent des Non-Lieux empiriques, que sont les espaces de circulation, de consommation et de communication, espaces marqués par l'éphémère et le passage. Leur inventaire compte aussi bien les installations nécessaires à la circulation accélérée des personnes et des biens (voies rapides, échangeurs, gares, aéroports) que les moyens 
de transport eux-mêmes (voitures, bus, trains ou avions), mais également les grandes chaînes hôtelières aux chambres interchangeables, les supermarchés ou encore les camps de transit, où se concentrent, souvent durablement, les réfugiés. Le Non-Lieu, selon Marc Augé, est donc tout le contraire d'une demeure, d'un lieu au sens commun $\mathrm{du}$ terme. Il se signifie par l'absence de subversion possible, de détournement. Il est exclusivement renvoyé à sa fonctionnalité pratique, à laquelle l'usager ne peut que consentir. Pour autant, ces espaces de transit peuvent gagner une qualité de lieux dans leur mouvance même et leur incessante circulation, ce dont témoigne l'émergence de lieux alternatifs à la lisière des centres.

La transition, comme passage graduel et changement d'état, requiert des lieux offrant cette aptitude et appelle des lieux transitionnels où se donne à voir une création continuée. Les lieux alternatifs mobilisent significativement des espaces de l'entredeux où se joue un imaginaire subversif entre réalité et virtualité. En 2011, l'ethnologue Agnès Jeanjean, réfléchissant avec l'archéologue Ingrid Sénépart aux modalités de l'habitat temporaire ${ }^{18}$, relève dans les pays riches la recrudescence de formes hétéroclites d'habitats, présentes dans les paysages ruraux et urbains. L'habitat précaire est certes largement relié à des situations économiques le justifiant, mais audelà, d'autres logiques sont à l'œuvre qui réinventent des formes légères et mobiles, inscrites dans un processus dynamique qui interroge les normes et les représentations en vigueur.

Depuis les années 2010, l'habitat transitoire évoque des réalités multiples et un éventail de publics, allant des grands exclus aux hypermobiles assumés. On assiste à une effervescence autour de projets d'urbanisme transitoire, gagnant une visibilité dans l'espace public. Cela englobe toutes les initiatives qui visent, sur des terrains ou bâtiments inoccupés, à réactiver la vie locale de façon provisoire, lorsque l'usage du site n'est pas encore décidé ou le temps qu'un projet s'y réalise. Ces lieux réinvestis temporairement suscitent l'innovation, la créativité et la mixité des usages, dans des espaces ouverts. Il s'agit de lieux co-construits par les habitants et le réseau associatif environnant. On a donc une sorte de fabrique urbaine renouvelée, liée à l'optimisation $\mathrm{du}$ foncier et porteuse de projets participatifs, comme le rappellent les urbanistes Cécile Diguet, Pauline Zeiger, Alexandra Cocquière ${ }^{19}$.

L'urbanisme transitoire advient ainsi en marge des projets immobiliers, dans les interstices que ceux-ci laissent. C'est pourquoi on y retrouve tous les espaces délaissés, les friches, les terrains vagues qui regroupent des réalités très dissemblables et mal définies. On voit comment les productions urbaines doivent faire avec des lieux désindustrialisés, des lieux laissés en déshérence du fait d'héritages insolubles, de négociations inabouties ou alors de sols pollués. Ces espaces étaient traditionnellement dévolus à des usages illicites, des squats provisoires, mais dorénavant se consolident à travers des projets spatiaux construits, délibérés, en partenariat avec les acteurs publics. Le réseau des tiers-lieux ${ }^{20}$ s'étoffe tout autant que celui des friches urbaines, lieux hybrides où se mêlent des activités veillant à maintenir des échanges locaux et des services de proximité.

Ces lieux ne sont plus nécessairement l'objet d'une relégation, ils ont gagné une reconnaissance institutionnelle, mais persévèrent comme processus créatif ouvert induisant des pratiques subversives. Au titre de ces espaces subvertis, le trottoir apparaît lui-même comme lieu hétérotopique «anti-consommatoire ${ }^{21}$ " via les encombrants et le glanage. La valeur contestataire de l'hétérotopie se donne à lire à 
travers ce circuit marginal des marchandises. De la même manière, Jean-Christophe Bailly, dans un chapitre de son ouvrage Le Dépaysement: Voyages en France intitulé "Légers jardins, à peine », observe comment les jardins ouvriers de Saint-Étienne qui perdurent sur fond de crise économique se donnent comme contre-espace :

« À la lutte et à l'imagerie de la lutte, ils opposaient une diversion... c'est une sorte d'utopie concrète aux contours incertains, non pas le système tout entier d'une refonte, mais des suites fragmentées de marques légères indiquant souplement, discrètement, une autre façon d'habiter la terre. »

On voit ainsi comment le lieu hétérotopique emprunte une diversité de formes possibles, voire contradictoires. L'inventaire foucaldien des productions hétérotopiques s'avère en effet protéiforme et en cela vertigineux, autant que l'est la multiplicité des contextes contemporains auxquels se voit rapporté le concept d'hétérotopie. Mais celui-ci permet de repenser la question de l'espace et du territoire comme lieu de projection de l'imaginaire, trop longtemps envisagée à travers la polarisation espace réel/espace utopique, absolument virtuel, sachant que l'utopie est bien un univers sans lieu. L'hétérotopie au contraire est un véritable contre-espace localisable qui au cœur de l'expérience ordinaire offre conjointement sa matérialité et sa capacité à mobiliser les ressources de l'imaginaire. Elle est en cela un lieu de contestation et d'inversion des propriétés des lieux ordinaires, mais promeut elle-même des pratiques concrètes.

À l'aune de cette contestation et de cette inversion, le caractère expérimental du projet hétérotopique s'incarne de manière foisonnante. Michel Foucault à ce titre, rappelonsle, a mis en exergue la nature ludique de ces contre-espaces en y rattachant explicitement la tendance enfantine à détourner par le jeu la concrétude des lieux dans lesquels l'enfant évolue (le grenier, la tente d'Indiens ou le fond du jardin). Mais n'eston pas en droit parfois de s'interroger sur la consistance sociopolitique réelle, par-delà cette dimension poétique, de ces configurations spatiales alternatives? L'hétérotopie n'en vient-elle pas à désigner une pluralité problématique de lieux et d'usages? Audelà, les contre-espaces hétérotopiques bénéficiant d'une reconnaissance institutionnelle manifeste ne risquent-ils pas de se voir évalués et légitimés par un pouvoir institué auquel ils n'offriraient plus tant une résistance, faute de conflictualité, qu'une altérité2 ${ }^{22}$ tout à fait acceptable?

Dans les Mots et les choses (1966), Foucault relevait la capacité consolatrice du récit utopique à laquelle il opposait le pouvoir inquiétant de l'hétérotopie, dont il relevait en l'occurrence les effets sur le langage ${ }^{23}$. L'hétérotopie interroge nos représentations communes à travers une inscription territoriale dont elle tire sa force. Les lieux alternatifs, les espaces transitionnels, viennent en ce sens incarner un processus dynamique entre inclusion et exclusion. L'hétérotopie en effet est à la fois dans une dépendance aux normes (la dimension institutionnelle des projets alternatifs est dorénavant un paramètre majeur) et révèle simultanément sa capacité de résistance à celles-ci (avec le risque toutefois d'une neutralisation, voire d'une instrumentalisation politique ou économique). Le contre-espace hétérotopique est de nature transitionnelle en ce qu'il décline aussi bien des espaces de transit à proprement parler que des espaces pour lesquels le passage, la circulation, est constamment assuré. La temporalité de ces lieux elle-même favorise l'interpénétration du temporaire et du durable, l'enjeu étant le maintien d'un processus de création continuée. 


\section{BIBLIOGRAPHIE}

AUGÉ Marc, Non-Lieux. Introduction à une anthropologie de la surmodernité, Paris, Seuil, « La Librairie du Xx siècle », 1992.

BAILLY Jean-Christophe, Le Dépaysement : voyages en France, Paris, Seuil, 2011.

BOULlant François, Michel Foucault, Penseur de l'espace, Conférence donnée le 15 janvier 2003 à

l'université Lille III. Groupe d'études « La philosophie au sens large », animé par Pierre Macherey, éd. numérique,

[URL : http://1libertaire.free.fr/Foucault49.html]

BURRET Antoine, « Démocratiser les tiers-lieux », Multitudes, 2013/1 (n 52), p. 89-97.

СНАВот Pascal, L’Âge des transitions, Paris, PUF, 2015.

DIGUET Cécile, ZEIGER Pauline, COCQUIÈRE Alexandra, «L'urbanisme transitoire : Aménager

autrement », Note rapide Territoires, n 741 de l'Institut Paris Région, Février 2017.

FOUCAUlt Michel, Les mots et les choses. Une archéologie des sciences humaines, Paris, Gallimard, 1966.

FOUCAULT Michel, « Des espaces autres », Empan, 2004/2, nº 54, p. 12-19.

FOUCAULT Michel, Dits et écrits, vol. IV 1980-1988, p. 752-762, Paris, Gallimard, 1994.

JEANJEAN Agnès, SÉNÉPART Ingrid (dir.) « Habiter le temporaire. Habitations de fortunes, mobiles et éphémères ", Techniques \& Culture, $\mathrm{n}^{\circ} 56,1^{\mathrm{er}}$ semestre 2011.

ROMAN Sébastien, « Hétérotopie et utopie pratique : comparaison entre Foucault et Ricœur », Le Philosophoire 2015/2 ( $\left.\mathrm{n}^{\circ} 44\right)$, p. 69-86.

ROUX Dominique, GUILLARD Valérie, « Le trottoir comme hétérotopie : exploration d'une nouvelle utopie contemporaine dans la consommation », 13 th International Marketing Trends Conference, janvier 2014, Venise, Italie (11 p.).

SABOT Philippe, Langage, société, corps. Utopies et hétérotopies chez Michel Foucault, Materiali Foucaultiani, mf/materiali foucaultiani, éd. numérique, 2012, I (1), p. 17-35.

[URL : https://halshs.archives-ouvertes.fr/halshs-00746742]

SERVOISE Sylvie, « Enfances dystopiques », 19 au 21 juin 2019, colloque international organisé par Sylvie Servoise (3L.AM, Le Mans-Université).

\section{NOTES}

1. «Des espaces autres ", conférence donnée au Cercle d'études architecturales à Paris le 14 mars 1967. M. Foucault n'autorisa la publication de ce texte, écrit en Tunisie la même année, qu'au printemps 1984 dans Architecture, Mouvement, Continuité, $\mathrm{n}^{\circ} 5$ (oct. 1984, p. 46-49), en parallèle de l'exposition berlinoise Idée, processus et résultat au Martin-Gropius-Bau (le musée des Arts décoratifs de Berlin). Ce texte sera repris à l'occasion de la parution posthume du recueil Dits et Écrits.

2. P. Chabot, L'Âge des Transitions, p. 27.

3. Ibid., p. 27. 
4. Dans son article «Utopies et hétérotopies chez Michel Foucault», le philosophe Philippe Sabot déplore la surexploitation dont a fait l'objet le concept d'hétérotopie dès la fin des années soixante, celui-ci s'apparentant dès lors moins à un outil d'analyse de l'espace qu'une clé universelle d'interprétation des espaces et des comportements urbains contemporains.

5. F. Boullant, Michel Foucault, Penseur de l'espace, conférence donnée le 15 janvier 2003 à l'université Lille III.

6. « Des espaces autres », p. 12.

7. Ibid., p. 13.

8. Ibid., p. 13.

9. Ibid., p. 15.

10. Ibid., p. 14.

11. Le succès des dystopies auprès du public adolescent s'est notamment vu analysé lors du colloque « Enfances dystopiques », organisé par l'université du Mans en 2019.

12. Ibid., p. 15.

13. Ibid., p. 17.

14. Ibid., p. 17.

15. Ibid., p. 17.

16. Ibid., p. 18.

17. M. Augé, Non-Lieux. Introduction à une anthropologie de la surmodernité.

18. A.Jeanjean, I. Sénépart (dir.), «Habiter le temporaire. Habitations de fortune, mobiles et éphémères ".

19. C. Diguet, P.Zeiger, A.Cocquière, «L'urbanisme transitoire: Aménager autrement », p. 2.

20. La notion de tiers-lieu a été introduite par le sociologue américain Ray oldenburg (1989). Elle fait référence à ces espaces intermédiaires, à mi-chemin entre espace de travail et domicile. A. Burret, « Démocratiser les tiers-lieux », p. 89.

21. D. Roux et V. Guillard, « Le trottoir comme hétérotopie : exploration d'une nouvelle utopie contemporaine dans la consommation ».

22. Nous empruntons les termes de conflictualité et d'altérité à Sébastien Roman, dans son article " Hétérotopie et utopie pratique : comparaison entre Foucault et Ricœur ».

23. M. Foucault, Les mots et les choses. Une archéologie des sciences humaines, p. 9.

\section{RÉSUMÉS}

À partir d'une reprise du concept d'hétérotopie forgé par Michel Foucault dans sa conférence «Les espaces autres » (1967), il s'agit de réfléchir à la question d'espaces matérialisés mettant en tension le passage du virtuel au réel dans une volonté de subversion des normes spatiales. En effet, l'hétérotopie comme lieu autre autorise la recherche d'espaces proprement transitionnels 
dont il importe d'interroger les formes tout autant que les projets qui pourraient s'y incarner. L'hétérotopie, contrairement à l'utopie (« emplacement sans lieu réel », dit Foucault), se donne comme lieu réel ou "contre-emplacement», qui offre sa matérialité et peut possiblement à travers sa valeur contestataire s'avérer tout à la fois transgressive et jouissive. Dans ce texte précurseur, Foucault pressent la part essentielle que les problématiques liées à l'espace vont prendre désormais et il pressent en cela les dynamiques œuvrant dans ce que le philosophe Pascal Chabot nomme L'Âge des transitions.

\section{AUTEUR}

\section{AGNÈS BESSON}

Docteure en philosophie, chargée de cours en Anthropologie-Ethnologie, Université Côte d'Azur 


\title{
L'écriture du regard géographique de Patrick Geddes (1854-1932) : le Collège des Écossais à l'université de Montpellier
}

\author{
Sabine Kraus
}

L'histoire de la science géographique est jalonnée d'autant de «procédures intelligentes " imaginées par les géographes pour répondre à la nécessité d'une représentation des réalités observées, ainsi que d'une compréhension globale du monde. La carte a été longtemps considérée comme l'outil privilégié de la géographie, entendue comme la représentation de la surface terrestre, ainsi que des frontières et des nations. À l'aube du xx siècle, la carte devient l'objet de critiques de la part de géographes qui étudient l'habitation humaine, car cet outil n'a pas la capacité de rendre compte de la dimension sociale des activités humaines et leurs interactions avec le territoire représenté. Patrick Geddes (1854-1932) (fig. 1), naturaliste, biologiste évolutionniste, sociologue et pédagogue écossais, apporte la synthèse la plus achevée de la réflexion sur les interrelations entre milieu naturel et milieu humain, ainsi que sur la manière de les représenter. Il s'attache à concevoir des "Thinking Machines ", diagrammes au potentiel hautement cognitif représentant l'ensemble de ces interrelations dans une vision unitaire, et permettant d'agir sur une réalité locale. Leur objectif est d'être un dispositif servant à organiser des actions citoyennes opérant sur l'évolution historique de la région et la construction de son futur. Dans l'étude des rapports entre espaces et sociétés, comme dans les multiples relations qui sous-tendent leur organisation au fil du temps, Geddes s'appuie tant sur l'approche historique des sociétés civiles du philosophe napolitain Giambattista Vico (1668-1744) que sur l'approche évolutive de la géographie historique du naturaliste berlinois Alexandre von Humboldt (1769-1859). 
Fig. 1. - Patrick Geddes assis sur la terrasse du Collège des Écossais.

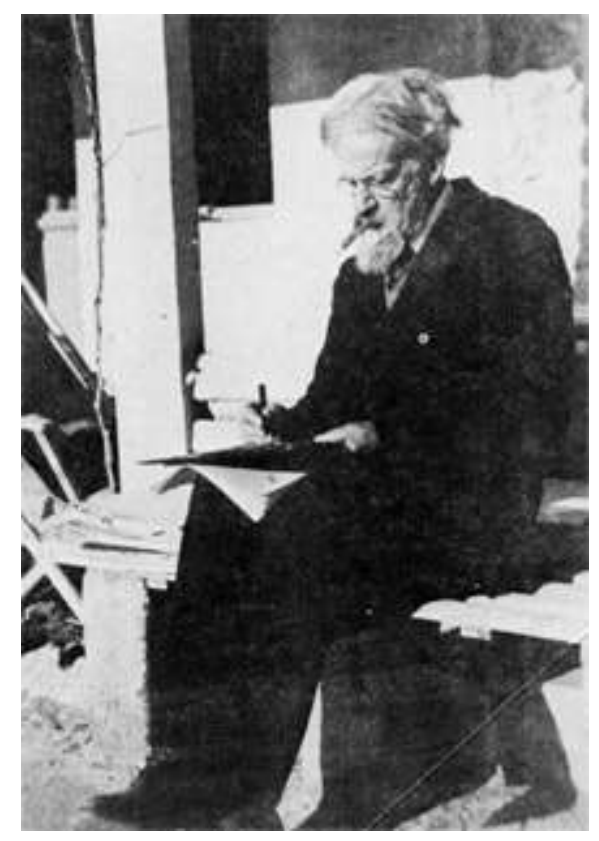

Photo de Philip Boardman, 1930, archives Benjamin Boardman. (c) Benjamin Boardman.

\section{Naissance de la géographie humaine et culturelle}

2 Vico et Humboldt font retour à Strabon pour élaborer leurs propres conceptions de la nature et de l'histoire, non comme entités opposées comme ce sera le cas à partir du $\mathrm{XIX}^{\mathrm{e}}$ siècle, mais en ne dissociant pas le monde naturel du monde des hommes, c'est-àdire les sciences des humanités. En effet, Strabon (63 av. J.-C. env. 25), géographe, historien et philosophe grec insistait sur ce grand principe que le géographe doit fixer ses regards sur les divisions naturelles de la Terre et sur la diversité des nations, plutôt que sur les limites que les caprices des gouvernements établissent pour un instant ${ }^{1}$.

\section{Une conception holiste de la nature humaine}

Le principe de Strabon sera suivi par Giambattista Vico dans son étude des choses humaines. Son esprit synthétique lui permet de réunir sous un principe commun des choses qui paraissent communément séparées entre elles ${ }^{2}$. Le domaine exploré par Vico est le monde civil, c'est-à-dire toutes les manifestations concrètes de la présence humaine. Il tire les principes d'une histoire universelle de la nature humaine à partir de celle des idées, des mœurs et des activités humaines. Vico reconnaît alors chaque société comme le résultat d'un processus historique de maturation situé dans un contexte déterminé, et établit une loi générale historique de la nature humaine en devenir, dont il trouve les principes dans la nature même de l'esprit humain et de ses modifications ${ }^{3}$. Cette loi établit pour chaque société le passage de l'imagination sensible à la raison pleinement développée. Ainsi, sa Science Nouvelle montre que chaque nation fixe son histoire avec le langage de son temps, et que le discours humain a son origine dans la mythologie et le langage naturellement poétique des nations païennes aux premiers temps de l'humanité. 
Dans les pas de Vico, Alexandre Von Humboldt s'intéresse à tout ce qui peut témoigner de l'histoire des peuples précolombiens lors de son exploration naturaliste des possessions espagnoles d'Amérique, de 1799 à 1804. Grâce à ses contacts avec les milieux érudits de Mexico, Humboldt avait été introduit à l'histoire du monde préhispanique, et avait rassemblé une riche variété de manuscrits indigènes, afin de retracer l'histoire des anciennes civilisations qui peuplaient le Mexique. Il s'attache à comprendre la signification de ce langage symbolique qui rend compte des mythologies et croyances précolombiennes, et qui inscrit l'histoire des civilisations premières d'Amérique latine avant l'arrivée des Espagnols. Et comme il le reconnaîtra :

«Nous pensons que c'est une idée belle et féconde que de considérer tous les peuples de la terre comme appartenant à une même famille, et de reconnaitre, dans les symboles chinois, égyptiens, persans et américains, le type d'un langage de signes qui est commun, pour ainsi dire, à l'espèce entière, et qui est le produit naturel des facultés intellectuelles de l'homme ${ }^{4}$.»

Comme Vico, Humboldt développe un savoir apte à amener à l'unité ce qui semble séparé et divers. Il associe expérience sensible et investigation scientifique à travers des représentations paysagères, qui nous font percevoir l'unité de la nature, ainsi que celle des champs disciplinaires investis. Ses célèbres coupes de versants sont des peintures vivantes qui donnent à voir à travers l'unité d'une image, la dynamique de l'ensemble des interactions entre les phénomènes naturels autant qu'humains, dans une approche écologique de la nature en tant que «réseau de la vie» reliant les animaux, les plantes, le climat, la géographie et la géologie.

\section{Une approche vitaliste de la géographie}

6 L'œuvre encyclopédique de Humboldt dessine les contours d'une géographie nouvelle qui en appelle, comme Aristote, à une vision synoptique de la nature, qui doit pouvoir être unifiée par le regard qui l'embrasse. L'école allemande de géographique fondée par le naturaliste berlinois, et suivie par son disciple Carl Ritter (1779-1859), ouvre la voie à une géographie humaine et culturelle, et sera le modèle sur lequel se fondera par la suite l'école française de géographie. Cette approche vitaliste de la géographie sera en outre le principal argument de Ritter dans sa critique de l'usage massif et dominant de cartes par les géographes. En témoigne l'analogie qu'il propose avec la médecine :

«Si le géographe voulait utiliser son recueil complet de cartes comme source principale pour administrer la preuve de sa science - et bon nombre de systèmes géographiques ne s'en sont pas privés - il en viendrait à commettre des erreurs encore plus monstrueuses qu'un physiologiste qui disséquerait un cadavre pour étudier le cœur vivant ou le phénomène de la vie lui-même, sans réaliser qu'il n'a devant lui qu'un corps privé de vie, une grossière caricature du corps humain ${ }^{5}$. »

7 Au tournant du xxe siècle, des géographes tels que Paul Vidal de la Blache (1845-1918), ou Élisée Reclus (1830-1905) reprennent la conception paysagère de Humboldt, et l'appliquent à l'étude des régions naturelles dans une approche anthropologique: l'homme comme être social agissant dans des milieux, évoluant dans des genres de vie et fabriquant des paysages ${ }^{6}$. La science géographique devient alors une histoire dynamique de l'habitation humaine, à travers les réponses collectives que les groupements humains ont apportées à leur environnement naturel, et qui sont liées au type d'habitat et à l'adaptation aux contraintes du milieu avec lequel les civilisations ont lutté pour pouvoir atteindre un plein développement. 


\section{La pensée visuelle de Patrick Geddes}

8 Patrick Geddes (1854-1932), biologiste évolutionniste, apporte la synthèse la plus achevée de la réflexion sur les interrelations entre milieu naturel et milieu humain. Sa naissance est contemporaine de la théorie de l'évolution de Darwin (1809-1882), ainsi que de l'affirmation dès 1850 d'une société industrielle et urbaine. Geddes rejette d'emblée une conception de la nature comme opposée et extérieure à la ville et à l'homme, et il étudie l'habitation humaine avec des géographes tels qu'Élisée Reclus et Pierre Kropotkine (1842-1921). Ce dernier examine les aspects de la vie animale qui regardent l'entraide entre animaux de même espèce, et considère l'entraide et la coopération entre êtres vivants comme un facteur d'évolution sociale. C'est cette réalité biologique qui guidera Geddes dans la conception de ses diagrammes, de manière à favoriser la coopération entre les êtres humains pour agir sur le devenir de leur milieu de vie. Fidèle à l'esprit encyclopédique et à la pensée visuelle de Humboldt, Geddes se saisira du concept de paysage qui permet de lier la connaissance scientifique à l'expérience sensible. Cependant, il s'éloigne de la représentation paysagère du naturaliste allemand pour s'orienter vers une écriture du regard géographique plus schématique, et formalisée en diagrammes.

9 En effet, à la recherche d'un champ plus vaste de compréhension de la vie humaine, Geddes a besoin d'une approche synthétique qui dépasse le cloisonnement des disciplines. À l'instar de Humboldt, il fait appel à Aristote quant à la nécessité d'une vision synoptique de la nature, de la région et de la ville comme un tout. De plus, le concept de la polis grecque implique une vision de la ville, non seulement comme une forme émergeant de la région dans laquelle elle se développe, mais également comme un phénomène culturel et spirituel ${ }^{7}$. La pensée visuelle de Geddes va le conduire à concevoir ses Thinking Machines, ou "figures de pensée ", comme des outils d'étude régionale, théoriques autant que pratiques. Elles sont une représentation démocratique et humaniste des connaissances géographiques et historiques, en vue de guider l'action locale des citoyens pour construire l'avenir de leur région comme un lieu de bien-être et de beauté. Ses diagrammes relient ainsi différents points de vue dans l'approche visuelle globale d'une nature vivante, immense réceptacle de labeur des générations qui nous ont précédées. Les « figures de pensée » de Geddes les plus commentées sont la «Valley Section » et la « Outlook Tower».

\section{Le diagramme de la " Valley Section »}

Le diagramme de la «Valley Section » (fig. 2) représente un type universel dans lequel la région est l'échelle adéquate pour analyser les relations entre la géographie du lieu, l'économie du terroir et le type de regroupement humain. 
Fig. 2. - Diagramme de la « Valley Section».

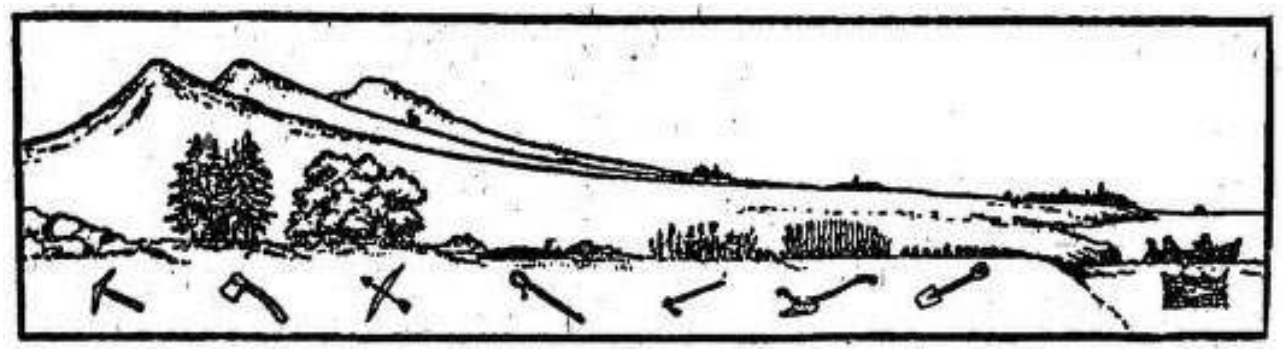

V. Branford et P. Geddes, The Coming Polity, 1917, p. 84

11 Le diagramme reprend la coupe transversale du géographe. Il suit la pente générale d'un paysage ordinaire, qui descend de la source vers la mer, et qui est façonné par le climat, les sols, et la vie végétale et animale correspondante. La gamme des activités humaines s'inspire de la classification des naturalistes, et se répartit selon les occupations naturelles reliées aux types d'habitats et à l'adaptation écologique au milieu. On peut ainsi suivre l'évolution des groupements humains depuis leurs simples origines jusqu'à l'accomplissement de stades plus complexes.

12 Le principe universel de la "Valley Section ", chargé des valeurs de tout le territoire vécu et construit par l'homme, permet ainsi de comprendre comment humains, artefacts et nature co-évoluent dans la constitution d'une unité régionale cohérente ${ }^{8}$.

\section{Le diagramme de la « Outlook Tower »}

13 Afin de pouvoir concrètement embrasser du regard le développement régional, Geddes conçoit le diagramme de la "Outlook Tower", pensé comme un musée géographique reliant par échelles successives la cité et sa région à l'ordre du cosmos, aussi bien qu'aux dimensions culturelles et spirituelles des civilisations. Tout comme sa «Valley Section », la "Outlook Tower » est un concept fondé sur l'association de la biologie aux sciences sociales. À la fois une idée et un bâtiment, la tour d'observation permet de déployer le schéma de la vallée dans une mise en œuvre de cette harmonie réelle des études à laquelle aspire Geddes dans un esprit holiste, et qu'il orientera toujours vers des applications et des résultats pratiques (fig. 3). 
Fig. 3. - « La Outlook Tower».

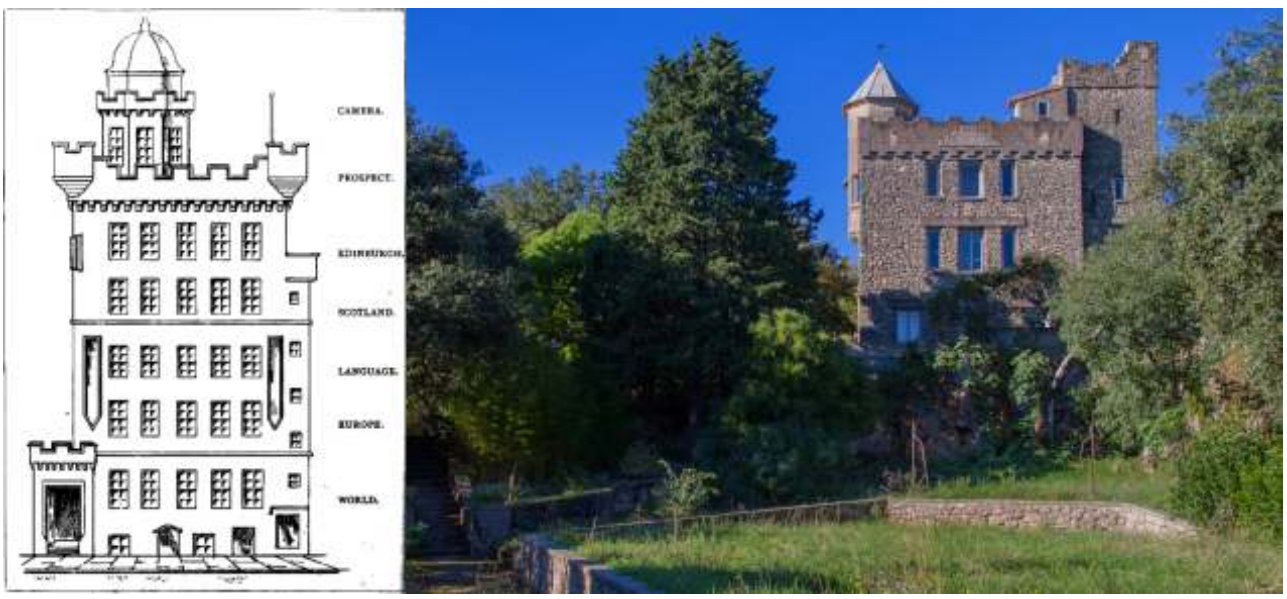

Diagramme de la « Outlook Tower », P. Geddes, Cities in Evolution, 1915, p. 324. Vue générale de la "Outlook Tower », façade Est, à l'à-pic de l'ancienne carrière, photo Gérard Pujol @).

Dans son école de la "Outlook Tower", premier laboratoire social pour les citoyens, toute étude et enquête régionale associant les connaissances naturalistes et humanistes mène à un service social fondamental, à un résultat pratique en termes d'un renouveau vitaliste de l'éducation citoyenne ouverte au plus grand nombre. Action et pensée, cité et université interagissent alors pleinement dans la vie, celle de la société et de ses membres, progressant mutuellement dans la construction de l'avenir. Nature et Civilisation sont ainsi unifiées à l'intérieur du concept de vie en évolution ${ }^{9}$. Cette conception tend en outre vers une véritable écologie de la vie à travers l'esthétique ordinaire de notre vie quotidienne.

15 À travers ces deux figures de pensée, le concept de la région typique est défini par Geddes comme «l'Eco-drame historique de la vie en évolution ». Il décrit sa coupe de vallée comme :

«Une image dans laquelle l'intérêt central sera historique, si nous entendons par historique le déploiement de cette interaction durable entre la société humaine et l'environnement géographique dans lequel chacun subit un changement continu et corrélatif, pour le meilleur ou pour le pire. C'est un drame de l'évolution dans lequel l'homme et la nature jouent un rôle épique ${ }^{10}$.»

16 Ainsi, le vitrail de la « Valley Section » réalisé à Edinburgh porte l'inscription suivante : Microcosme de la Nature, Siège de l'Humanité, Théâtre de l'Histoire, Eutopie du Futur.

\section{Les Jardins symboliques du Collège des Écossais à Montpellier}

17 Cependant, les termes de cette inscription renvoient également à l'une des figures de pensée de Geddes qui a été largement ignorée. En effet, ses constants allers-retours entre le réel et sa représentation le mèneront à la création de Jardins symboliques au Collège des Écossais, première résidence étudiante internationale qu'il fonde à l'université de Montpellier en 1924, à l'âge de $70 \mathrm{ans}^{11}$. Ce site exceptionnel est de fait l'héritage symbolique et vivant de l'œuvre de toute sa vie. Ici, c'est la matérialité des jardins qui est le support d'une procédure intelligente, guidée par une lecture de la situation géographique de la France en Europe comme l'ellipse reliant la Méditerranée à l'Atlantique Nord. Les Jardins seront agencés en un théâtre de mémoire des cultures 
et mythologies méditerranéennes et celtes, ainsi que des sciences et philosophies européennes. En effet, pour Geddes :

«Le plus humble jardinier est un steward de Mère Nature, dans la splendeur sans fin de ses saisons. Et le jardinier peut aussi être un penseur, écrivant ses pensées en formes et couleurs vivantes en place de simples mots imprimés ${ }^{12}$."

Nous retrouvons ici l'apport de Vico et Humboldt concernant la "sagesse poétique " des premiers âges de l'humanité, dont la nature commune est celle du mytho-poétique. À travers la rigueur du poète et l'imagination du scientifique, l'avancée intellectuelle et morale de l'humanité est actualisée dans la réalité concrète du Collège des Écossais. De fait, le site est pensé comme un tout organique dans sa totalité, offrant toutes les ressources d'un environnement historique et naturaliste à travers une expérience de vie active. Comme le précise un ancien résident du Collège, Philip Boardman à propos des « Jardins de pensée » de Geddes :

« Nous étions immergés dans la nature et l'histoire, et non dans des livres, nous y apprenions en vivant... Le terrain du Collège des Écossais était aménagé en une configuration des plus inhabituelles de l'histoire et des connaissances humaines ${ }^{13}$. »

La mise en espace de la pensée symbolique de Patrick Geddes sur les hauteurs du Plan des Quatre Seigneurs est donc un véritable texte à lire.

\section{L'université médiévale et la Auld Alliance}

Geddes avait été attiré par Montpellier en raison de la tradition de longue date de son université comme capitale de la botanique dès le Moyen Âge, à travers l'enseignement de son illustre faculté de médecine qui rayonna sur l'Europe durant de longs siècles. L'esprit de sa résidence étudiante internationale emprunte à l'université médiévale la création de Collèges destinés à héberger des étudiants pauvres. En 1369, Urbain V, ami des arts et des sciences, fonde le Collège de Mende, destiné à héberger douze étudiants en médecine originaires du diocèse natal du pape. L'Écosse avait également son Collège à Paris, fondé en 1325 par David, évêque de Murray, pour y loger quatre étudiants pauvres écossais, qui bénéficiaient, grâce au traité de la Auld Alliance, de la nationalité française. C'est donc à Montpellier que Geddes décide de réédifier le Collège médiéval des Écossais, et de revitaliser les liens d'amitié entre la France et l'Écosse, scellés par le traité d'alliance de 1296. Le Collège de Geddes s'inspire à la fois des Bénédictins pour l'étude et la spiritualité, et des Franciscains pour leur ouverture sur le monde laïc, afin de se mettre au service des autres ${ }^{14}$. Pensé comme lieu d'études et de vie, le but principal de son Collège sera d'offrir aux résidents une culture générale associant sciences et humanités, trop dispersées dans les études universitaires.

Deux bas-reliefs apposés sur les façades de la « Outlook Tower » symbolisent les racines médiévales de son Collège. À l'est, l'inscription "Vivat Academia » (Vive l'université) renvoie au chant étudiant le plus célèbre, le Gaudeamus Igitur (Réjouissons-nous donc) datant du XIII e siècle. Au sud, le blason portant la devise «Vivendo Discimus » (c'est en vivant que nous apprenons) fait référence à la Auld Alliance, et l'on y retrouve le lys de la France et les chardons de l'Écosse (fig. 4). 
Fig. 4. - Les deux bas-reliefs médiévaux de la « Outlook Tower ».

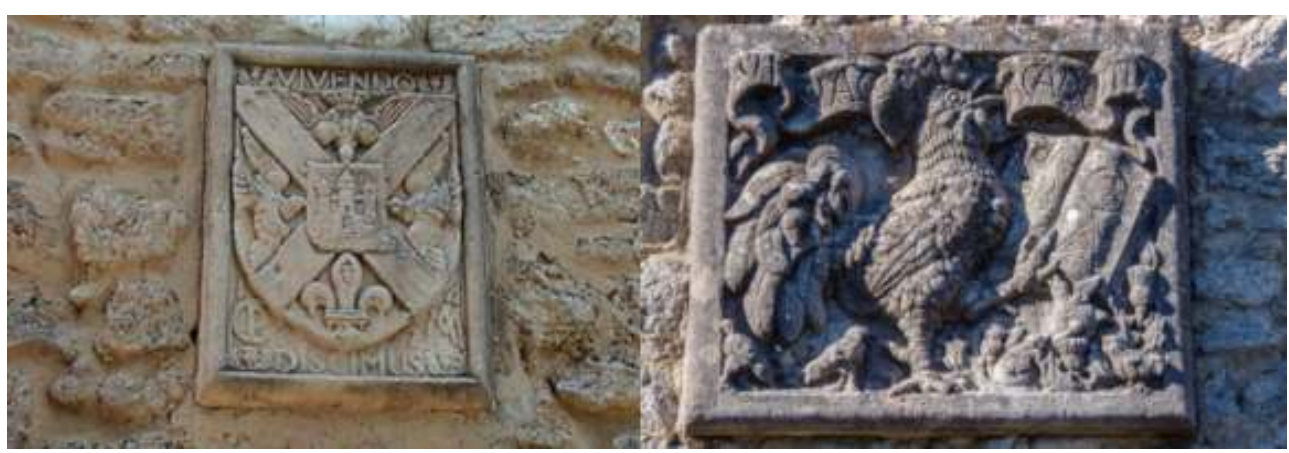

Façade Sud, Vivendo Discimus : photo Sabine Kraus @ @. Façade Est, Vivat Academia, photo Gérard Pujol (C).

\section{Les Jardins de l'Antiquité}

Pour construire son Collège, Geddes prend modèle sur le Mouseîon, la colline consacrée aux Muses face à l'Acropole, et choisit les hauteurs d'une colline dominant la plaine montpelliéraine. Les jardins se développent en pente à partir du sommet, et remodèlent le terrain par la réalisation d'une série de terrasses en restanques typiques des régions méditerranéennes. L'entrée principale, au bas du coteau, nous fait accéder aux Jardins de l'Antiquité par l'Allée des Philosophes bordée de cyprès. Plus haut sur le versant se trouve l'espace de l'Olympe, avec le cercle des dieux et déesses grecs. En guise de statuaire, Geddes choisit un groupe de résidents personnifiant les statues sur leur piédestal. Pendant ce temps, il discourt longuement sur la signification sociologique de la mythologie grecque, en expliquant que de Cupidon à Zeus, et d'Artémis à Demeter, dieux et déesses symbolisent les sept âges du développement de la vie de l'homme et de la femme ${ }^{15}$. L'espace de l'Antiquité s'achève avec le Jardin Grec jouxtant la façade ouest du pavillon des Écossais, construit au sommet du versant (fig. 5). 
Fig. 5. - Allée des Philosophes.

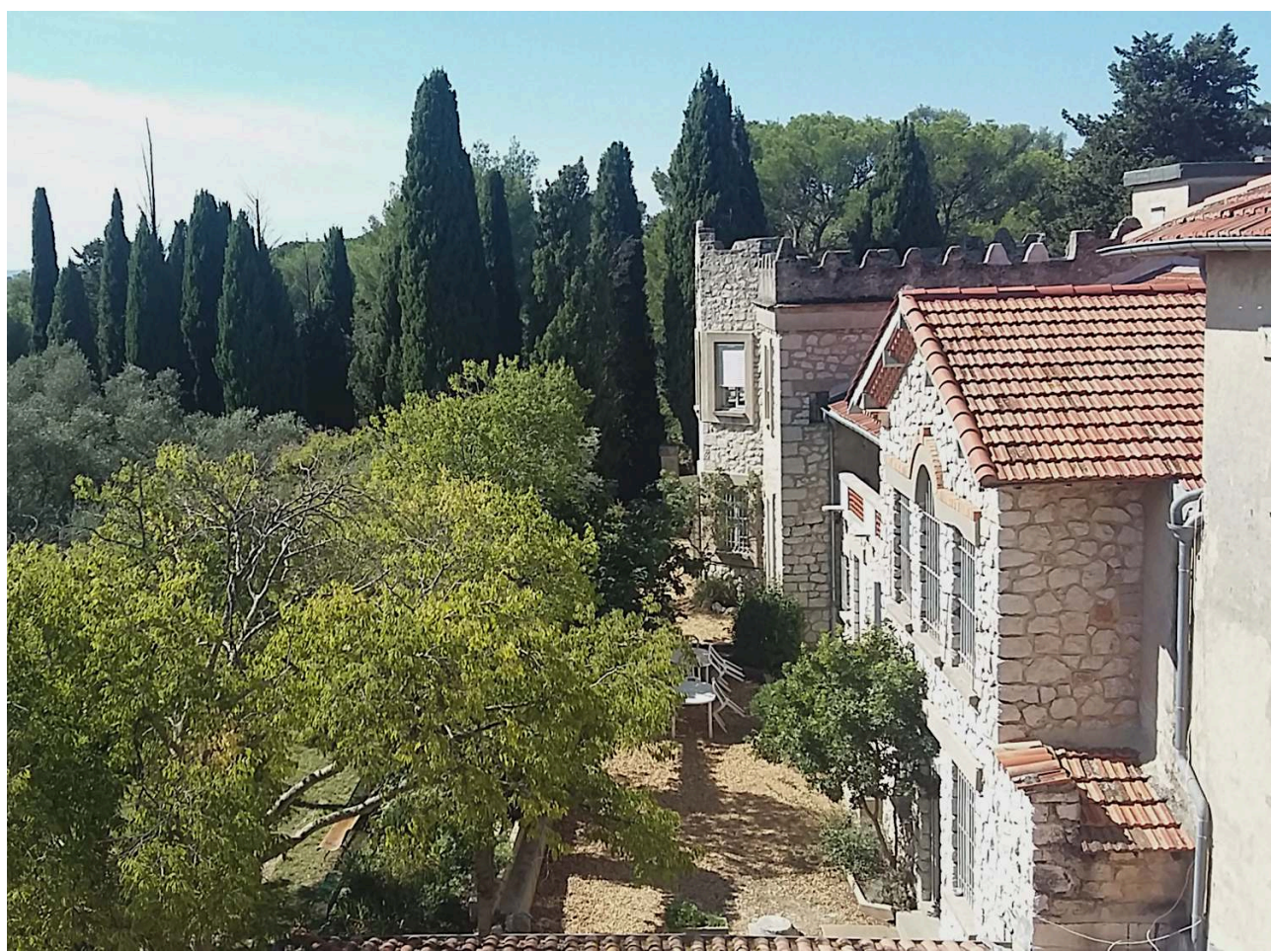

Photo Sabine Kraus (c).

L'enseignement péripatéticien que Geddes délivre dans ses jardins renvoie à Aristote, non seulement au Lyceum, mais également à la notion de thaumaston, c'est-à-dire l'émerveillement devant la beauté de la nature. Et pour Geddes, comme pour Aristote, le fait de la beauté ne se limite pas à la nature, la science elle-même en tire sa valeur.

\section{Les Jardins de la Renaissance}

La Grotte des Druides et le Jardin de la Renaissance avec la table ronde du roi Arthur, situé à quelques pas, bordent la façade sud du corps de bâtiment construit en 1928. La Renaissance celte est un mouvement culturel dirigé par Geddes à Édimbourg dans les années 1890, dans la visée de revitaliser les anciens mythes et légendes, dont les figures emblématiques sont le roi Arthur et le barde Ossian. Arthur et Ossian symbolisent la renaissance et la régénération. Comme Geddes le déclare, il s'agit de «la jeunesse apparaissant dans sa force et l'ouverture des possibles ${ }^{16} »$. La légende d'Arthur a également de fortes connotations de revitalisation pour Geddes, comme «l'aventure spirituelle décisive de la jeunesse, et le juste service de l'âge et de l'expérience pour la guider ${ }^{17} »$. Geddes nous montre son attachement à la valeur du passé dans la construction de l'avenir, tant au niveau abstrait que théorique, qu'au niveau des relations personnelles et sociales ${ }^{18}$.

La grotte des Druides nous rappelle que les prêtres du chêne savaient lire dans le grand livre de la nature. Ils en connaissaient les lois, par leur pratique de ce que les Grecs appelaient physiologie. Le barde Ossian, surnommé l'Homère celte, chante dans ses poèmes les hymnes à l'idéalisme créatif, portant les quatre dons de la vie aux hommes. Tout d'abord, la branche foisonnante de feuilles, de fleurs et de fruits de l'arbre de vie : la vie simple et le travail du peuple. Le prochain porte la Coupe : pour la joie de vivre 
dans sa prospérité. Le suivant regarde dans son Cristal magique de la pensée, dans lequel l'émotion, la raison et l'intuition créent constamment de nouvelles visions. Vient enfin l'hymne qui porte l'épée: pour l'idéalisme dans l'action, et la justice dans la règle ${ }^{19}$ (fig. 6).

Fig. 6. - La Grotte des Druides.

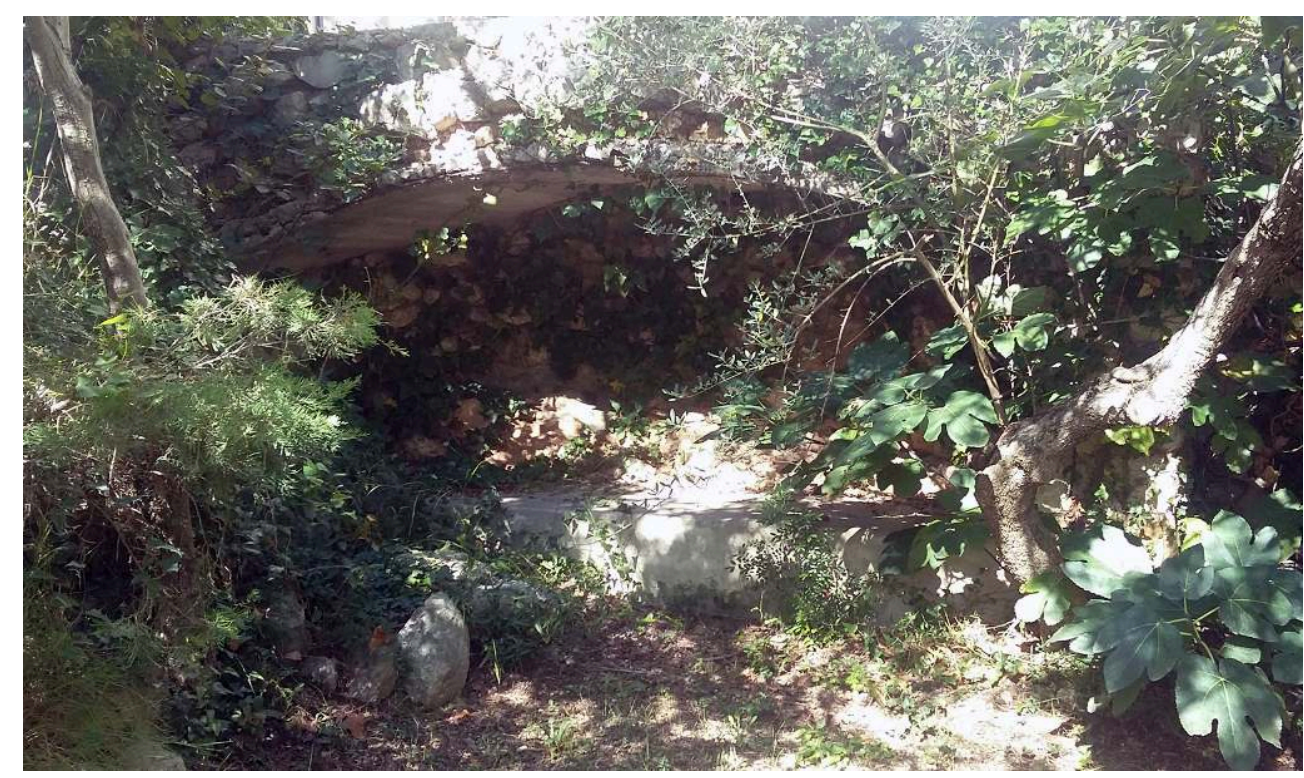

Photo Sabine Kraus (c).

Ainsi, les Jardins grecs et celtes de Geddes nous parlent de l'âge des dieux et de l'âge des héros des premières civilisations, et ils manifestent sa volonté de les restaurer parmi nous en tant qu'idéalisations de la vie humaine, non moins vitales pour le bien-être organique que le gîte et le couvert ${ }^{20}$.

\section{La Terrasse de la Classification des Sciences}

La classification des sciences d'Auguste Comte (1798-1857), philosophe natif de Montpellier, donne son nom à la terrasse principale surplombant la Promenade de la Synthèse. Le dallage de cette terrasse reprend le diagramme de Geddes, que l'on retrouve au sommet de la "Outlook Tower». Des axes symbolisent les mathématiques, une balance la physique, un scarabée la biologie, un livre ouvert la sociologie. Puis, en contrepartie, leurs applications : l'économie et la politique, la médecine et l'agriculture, la technique et enfin la métrique. Cette classification place la sociologie, l'économie et la politique au sommet de toutes les connaissances : elle pose une théorie de la vie en évolution fondée sur le passage de la biologie à la sociologie, et le passage des sciences naturelles à l'économie. Geddes, à travers l'unité de son diagramme, nous montre que la philosophie positive de Comte est un système dans lequel les relations entre les conceptions, les connaissances, leurs rapports et leurs coordinations importent, car rien ne tient que par l'ensemble. À l'instar de Vico, Auguste Comte en appelle à une science de la société fondée sur l'étude de l'histoire, dans la visée de maitriser les lois de l'esprit humain tirées d'observations réfléchies sur l'histoire de l'humanité, et de trouver les guides d'une saine éducation ${ }^{21}$. De même, pour Geddes, cette dernière est la clé d'une réorganisation et d'une régénération sociale, car le biologiste écossais 
considère l'éducation comme un facteur d'évolution. À travers son engagement militant dans l'éducation populaire pour une diffusion de la connaissance au plus grand nombre, il s'attache aux valeurs morales et spirituelles de la philosophie positive dans la réorganisation de la société (fig. 7).

Fig. 7. - Le diagramme de la Classification des Sciences.

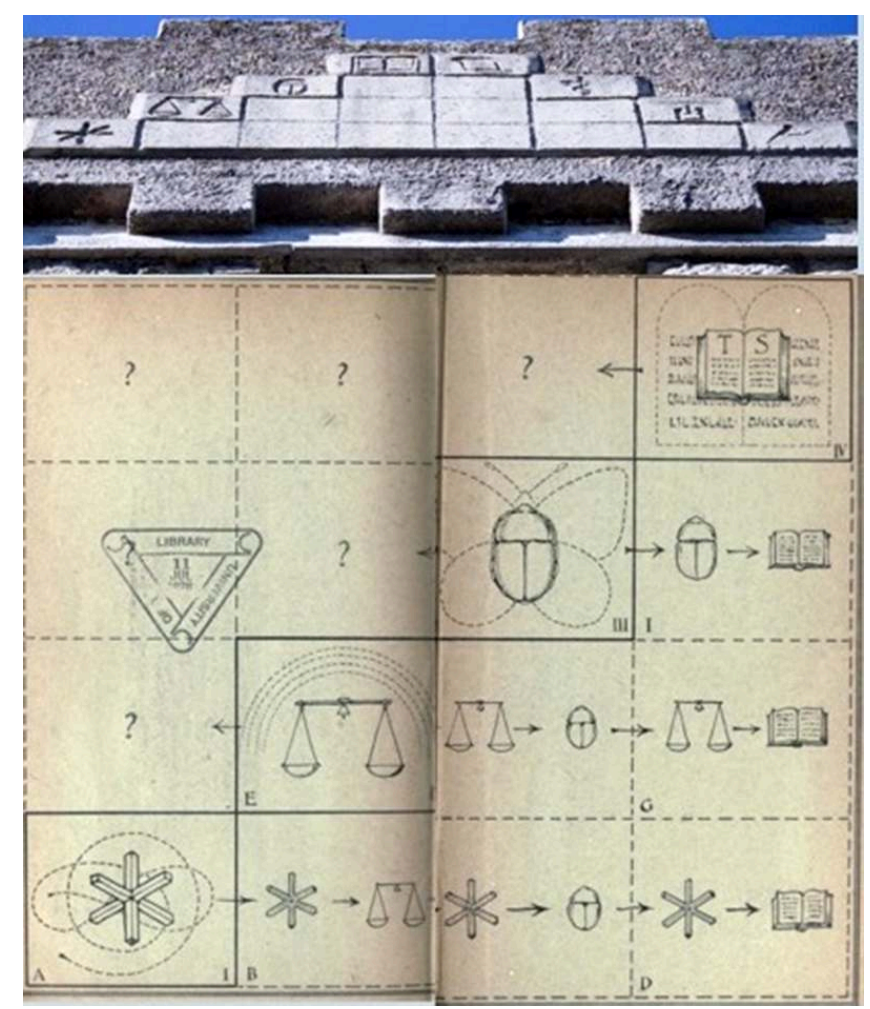

Le bas-relief de la Classification des Sciences, au sommet de la façade Est de la « Outlook Tower », photo Gérard Pujol ( ). Troisième de couverture de Life, Outlines of General Biology, P. Geddes et A. Thomson, 1931.

\section{L'Urne de l'Économie Métaphysique}

L'Urne de l'Économie Métaphysique symbolise le discours le plus célèbre de Geddes (fig. 8). Il s'agit de la conférence d'adieu qu'il délivre en 1919 à l'université de Dundee, où il occupait la chaire de botanique, avant de partir en Inde pour inaugurer la chaire de sociologie à l'université de Bombay. La pensée économique du biologiste écossais se révèle ici dans toute sa portée écologique.

«Qui s'attarde à penser aux feuilles ? La feuille est pourtant le principal phénomène et produit de la vie : ce monde est vert, et c'est grâce à la vie végétale que nous vivons. Étrangement, certains s'imaginent vivre grâce à l'argent. Ils croient l'énergie générée par la circulation de la monnaie. Mais le monde n'est qu'une vaste colonie de feuillages, qui pousse et élabore un humus de feuilles, pas un simple substrat minéral : et nous ne vivons pas du tintement des pièces, mais de la beauté de nos récoltes ${ }^{22}$.» 
Fig. 8. - L'Urne de l'économie Métaphysique.

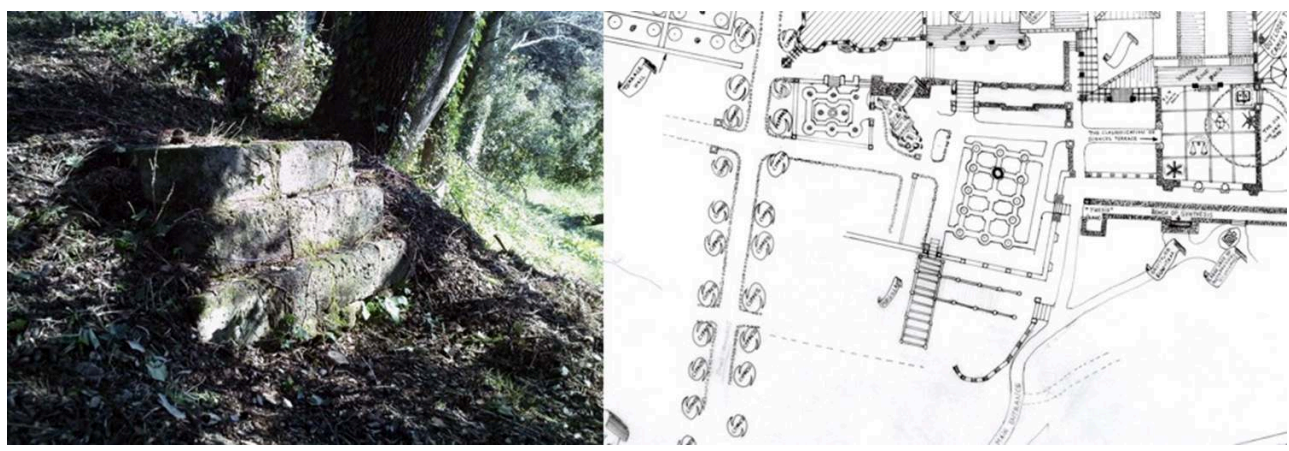

Photo du piédestal de l'Urne de l'Économie Métaphysique, découverte archéologique faite lors du premier chantier-école d'éducation au patrimoine culturel et d'activités de restauration des jardins en 2017 (conception du projet pédagogique et coordination, Sabine Kraus).

Photo Sabine Kraus ( C. Extrait du plan du Collège des Écossais dessiné par Benjamin Boardman et Philip, son père et ancien résident, d'après le projet original de Patrick Geddes. @ Boardman.

29 Geddes a toujours insisté sur le rôle essentiel de l'étude du passé dans le temps présent pour penser l'avenir. En associant sciences et humanités dans ses Jardins symboliques, il recompose un passé historique devenu une histoire idéale universelle qui s'incarne dans la réalité concrète de son Collège.

Véritable dispositif de réalité augmentée associant rationalité scientifique et imagination poétique, le Collège de Geddes est un agencement de procédures intelligentes conçues comme des vecteurs de signification et de valeurs dans un espace commun, soulignant leur dimension pédagogique, culturelle et sociale. De fait, pour Geddes, l'articulation du réel, et sa représentation, figure la modalité spécifique de la construction des relations entre les humains et le monde qu'ils habitent. En effet, ses productions ont des caractéristiques géographique, écologique et historique, en intégrant les soubassements sociaux, environnementaux et économiques d'un développement durable. Leur visée est l'éducation des habitants afin qu'ils puissent se saisir collectivement du devenir de leur région. Elles sont à la fois une «figure de pensée » qui a la capacité d'évoquer l'unité terrestre et la diversité qu'elle englobe, et une réalité concrète dont on peut faire l'expérience, participant ainsi d'une connaissance totale de l'homme et du monde dans un vibrant hommage au Web of Life de Humboldt (fig. 9). 
Fig. 9. - Vue générale des Jardins symboliques et du Collège des Écossais.

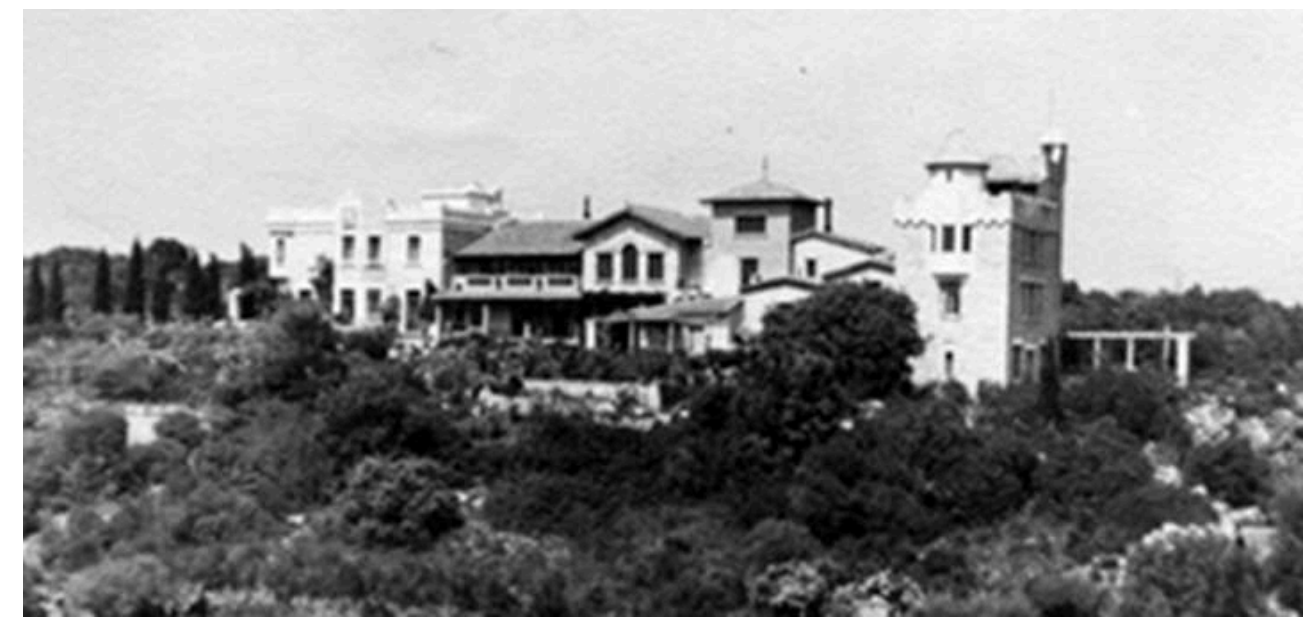

Photo de Philip Boardman (c), 1936, archives Benjamin Boardman.

\section{BIBLIOGRAPHIE}

BOARDMAN Philip, Patrick Geddes: Maker of the Future, Chapel Hill, The University of North Carolina Press, 1944.

BRANFORD Victor and GEDDES Patrick, Our social inheritance, The Making of the Future series, London, Williams and Norgate, 1919.

BRANFORD Victor and GEDDES Patrick, The Coming Polity, The Making of the Future series, London, Williams and Norgate, 1917.

DUSSIEUX Louis, Géographie générale, Paris, Jacques Lecoffre et Cie, Libraires-Éditeurs, 1866. FERGUSON Megan, Patrick Geddes and the Celtic Renascence of the 1890s, thèse de doctorat en philosophie de l'histoire, Dundee, Université de Dundee, Écosse, janvier 2011.

GEDDES Patrick, The Scots College Montpellier, Montpellier, Imprimerie de la Charité, 1928.

GEDDES Patrick, The Masque of Learning and its many Meaning, devised and interpreted by Patrick Geddes for the semi-jubilee of University hall, Edinburgh, Patrick Geddes and Colleagues, Outlook Tower Edinburgh, 1912.

GEDDES Patrick and THOMSON Arthur, Life, Outlines of General Biology, London, Williams and Norgate, 1931.

HUMBoLDT Alexandre von, Vues des cordillères et monuments des peuples indigènes de l'Amérique, Paris, tome second, chez N. Maze, Libraire, 1824.

KRAUS Sabine, « Aristotélisme, darwinisme et holisme chez Patrick Geddes », Espaces et sociétés, vol. 167, n 4, 2016, p. 121-135. 
MACDONALD Murdo, Excerpts from Patrick Geddes's farewell lecture to his Dundee students, 1919, 2007, publié en ligne sur : https://murdomacdonald.wordpress.com/patrick-geddes-farewell-lectureto-his-dundee-students-1919/

PETIT Annie, « Des sciences positives à la politique positiviste », Trajectoires positivistes, 1798-1998, Paris, L'Harmattan, 2003, p. 87-116.

PONS Alain, « Nature et histoire chez Vico », Les Études Philosophiques, vol. 16, n 1, 1961, p. 39-53.

RITTER Carl, « Introduction à la géographie générale comparée », traduction de Danielle NicolasObadia, Cahiers de géographie de Besançon, $\mathrm{n}^{\circ}$ 22, Presses Universitaires de Franche-Comté, 1974.

SANGUIN André-Louis, Vidal de la Blache, un génie de la géographie, Paris, Éditions Belin, 1993.

VICo Giambattista, Vici Vindiciae, Paris, Éditions Allia, 2004.

\section{NOTES}

1. L. Dussieux, Géographie générale, p. 83.

2. G. Vico, Vici Vindiciae, p. 47.

3. A. Pons, « Nature et histoire chez Vico », p. 46.

4. A. von Humboldt, Vues des cordillères et monuments des peuples indigènes de l'Amérique, p. 306-307.

5. C. Ritter, Introduction à la géographie générale comparée, p. 61-62.

6. A.-L. Sanguin, Vidal de la Blache, un génie de la géographie, p. 319.

7. S. Kraus, « Aristotélisme, darwinisme et holisme chez Patrick Geddes », p. 133.

8. En 1925, Geddes rédige un article dans lequel il expose l'évolution des occupations naturelles à travers la transformation des activités de chasse et de pâturage vers des sociétés commerçantes. Voir «The Valley Plan of Civilization », p.288-290, p. 322-323-325.

9. P. Geddes and A. Thomson, Life, Outlines of General Biology, p. 1384-1387.

10. V. Branford and P. Geddes, The Coming Polity, p. 77.

11. Le site dans sa totalité a été inscrit au titre des Monuments historiques le 19 décembre 2013, suite aux trois années d'investissement de la part de Sabine Kraus (travail de recherche, organisation de colloque, exposition, campagne européenne de demande de protection).

12. P. Boardman, Patrick Geddes: Maker of the Future, p. 446.

13. Ibid., p. 447.

14. P. Geddes, The Scots College Montpellier, p. 5.

15. P. Boardman, Patrick Geddes: Maker of the Future, p. 448.

16. P. Geddes, The Masque of Learning (...), p. 32.

17. Idem.

18. M. Ferguson, Patrick Geddes and the Celtic Renascence of the 1890s, p. 91-93.

19. P. Geddes, The Masque of Learning (...), p. 31.

20. V. Branford and P. Geddes, Our social inheritance, p. 22. 
21. A. Petit, «Des sciences positives à la politique positiviste », p. 9.

22. M. Macdonald, Excerpts from Patrick Geddes's farewell lecture to his Dundee students.

\section{RÉSUMÉS}

Le Collège des Écossais, première résidence étudiante internationale de France, est fondé à l'université de Montpellier en 1924 par Patrick Geddes (1854-1932), biologiste évolutionniste, sociologue et pédagogue écossais. Ce site exceptionnel, conçu comme lieu d'étude et de vie, est l'héritage symbolique et vivant de l'œuvre de toute sa vie. La valeur et l'importance des sciences biologiques, en tant que lien essentiel entre les sciences et les humanités, ont enraciné Geddes dans sa conviction de l'unité fondamentale de la nature et de la culture. En s'appuyant sur la pensée visuelle de Humboldt, Geddes met en espace sa réflexion sur les relations homme-nature au moyen de diagrammes et de jardins symboliques, qui manifestent de constants allers-retours entre le réel et sa représentation. Au-delà de la représentation d'un territoire, c'est bien sa théorie de la vie en évolution unifiant nature et civilisation qui fait l'objet d'une représentation, à travers des images qui s'actualisent dans la réalité concrète et quotidienne de notre existence.

\section{AUTEUR}

\section{SABINE KRAUS}

Chercheur en histoire des sciences et de la médecine, doctorante de l'EHESS 


\title{
Paysage sous-marin et géoarchéologie littorale : le cas de pointe de Porzh Karn en Bretagne (Penmarch, Finistère)
}

\author{
Yves Petit-Berghem et Gaëtan Jolly
}

\begin{abstract}
Le paysage sous-marin est une notion qui fait débat dans la communauté scientifique, elle interpelle aussi le citoyen comme le politique, car nous sommes dans un domaine qui touche au réel - la mer et les océans -, des masses d'eau constituant une immense ressource permettant d'en faire des hauts lieux de nature et de biodiversité. Un domaine qui peut aussi être celui du virtuel, car ce milieu marin n'est exploré que depuis peu de temps et la question de son paysage renvoie aussi à un imaginaire, à une infinité d'approches relevant des sciences et des pratiques. Le sujet appelle une nécessaire hybridation des champs. Par ailleurs, la notion traverse les champs disciplinaires et les dépasse ; le paysage de l'archéologue n'est pas celui du géographe, ou de l'écologue, ni celui de l'artiste ou du paysagiste, car les méthodes et les outils pour le définir et l'analyser ne sont pas identiques ${ }^{1}$. Dans un premier temps, nous aborderons une notion qui se nourrit des disciplines, mais s'en affranchit aussi. Parallèlement, nous montrerons que perception et représentation des paysages sousmarins ne peuvent être dissociées, car elles définissent les conditions d'existence d'un objet qui est aussi un sujet convoquant les hommes et les sociétés. Dans un second temps, un cas d'étude sera étudié appliqué à l'archéologie littorale; nous montrerons l'utilité d'une analyse paysagère sous-marine afin d'améliorer la connaissance d'un concept qui n'est pas encore opératoire.
\end{abstract}




\section{Le paysage sous-marin : un concept émergeant à la croisée des disciplines}

\section{Une notion traversière}

2 Le concept de paysage a donné lieu à de nombreux débats épistémologiques et réflexifs contribuant à renouveler les approches et à faire avancer la connaissance d'une notion complexe traversée par les disciplines. Les paysages littoraux ont fait l'objet de visions multiples et d'approches hybrides croisant les caractéristiques à la fois physiques et anthropiques des espaces côtiers. La part belle donnée aux analyses systémiques a montré l'articulation et l'imbrication entre des problématiques et des enjeux géopolitiques et environnementaux associant les échelles globales et locales, en s'appuyant sur les outils habituellement mobilisés par la communauté scientifique (cartographies, systèmes d'information géographique, images et croquis en deux ou trois dimensions, photographies, etc.). Traditionnellement, les travaux des chercheurs ont privilégié les portions terrestres des littoraux et se sont moins intéressés aux espaces maritimes (mers et océans) alors qu'ils constituent une immense ressource permettant d'en faire des réservoirs de biodiversité2.

3 Cette frange littorale sous-marine n'en constitue pas moins un espace étudié par les chercheurs qui y trouvent un objet d'étude intéressant. Les biologistes la définissent comme une mosaïque identifiable de biotopes organisés spatialement et de ses biocénoses $^{3}$ associées. Cet espace se résume dès lors à des notions d'unité écologique, faunistique, floristique, géomorphologique et topographique. Les géologues y voient des formes de relief associées à des matériaux transitant en mer. Les géographes se sont d'abord intéressés à la morphologie littorale, puis à la cartographie des fonds marins leur permettant d'identifier des formes et d'appréhender la dynamique du système côtier ${ }^{4}$. L'approche naturaliste les a conduits à analyser la couverture végétale et à travailler avec des biologistes marins en établissant une lecture d'unités biogéographiques en interaction les unes avec les autres. Plus récemment, ils en ont livré une définition plus anthropocentrée plaçant l'homme au cœur d'un processus de territorialisation où se déploient des pratiques liées à la richesse patrimoniale et identitaire du territoire observés. Territoire encore méconnu, la frange littorale sousmarine se structure socialement et économiquement autour d'images et de représentations dans lesquelles se mêlent esthétisme, émotions et sensibilités. Si cette mise en scène confère une valeur d'existence au paysage sous-marin, elle n'en donne pas une définition claire, qui soit commune et acceptée par la communauté scientifique.

4 Cette absence de consensus n'est pas un frein aux prospections, car le paysage sousmarin est d'abord celui d'une pratique ou d'une action. En effet, ce paysage se vit, et l'expérience de la plongée est importante car elle débouche sur la perception et la représentation, mais aussi sur les difficultés à saisir un paysage, à l'appréhender et à l'analyser.

\section{Perception et représentation d'un paysage difficile à saisir}

5 La notion de paysage sous-marin se différencie de celle du milieu, car tout paysage doit passer avant tout par le filtre de la perception, qu'elle soit visuelle, ou encore sonore, 
olfactive. Ce sont les sens qui entrent en action et ces sens sont investis par les populations qui vont faire le paysage.

6 Or, deux difficultés se posent pour le monde sous-marin : la question de la perception, parfois réduite ou impossible si on s'en tient à la perception visuelle, et la question de l'accessibilité, le paysage sous-marin n'étant pas immédiatement accessible pour tous et cette accessibilité étant aussi conditionnée par la profondeur et la clarté des eaux. En effet, force est de constater que la profondeur et la turbidité des fonds limitent bien souvent le champ de prospection; le paysage n'est donc pas toujours une réalité tangible même si des formes paysagères, plus ou moins spatialisables, peuvent être identifiées lors d'exercices de plongée par exemple.

7 Pour ceux qui ne pratiquent pas la plongée et l'observation directe des fonds, le sujet ne peut être abordé qu'indirectement au travers de la représentation, car chacun se fait aussi une image de la mer à partir de son expérience, de sa culture personnelle et de ses croyances, mais peut être également influencé par les images et les discours véhiculés par la société.

8 Cette représentation du paysage sous-marin s'est construite dans le temps, et montre un rapport des hommes à la mer très ambivalent, entre la crainte ou la répulsion, ou alors la curiosité, le désir, voire la fascination. Ces représentations sont véhiculées dans les œuvres littéraires ou artistiques, depuis l'Antiquité jusqu'à nos jours ${ }^{6}$. La fascination pour ce paysage, mais également son caractère fictif sont bien retranscrits par des auteurs comme Jules Verne qui met en récit ce paysage en mêlant sciences, techniques, et imaginaire ${ }^{7}$.

9 Cette représentation trouve un écho auprès des artistes et des photographes qui, dès le début du $\mathrm{Xx}^{\mathrm{e}}$ siècle, vont photographier ce monde sous-marin ; dès lors, la connaissance de ce paysage va franchir un pas, avec des clichés photographiques qui seront au fil des années plus nombreux et de qualité, et également des films qui vont donner au paysage une dimension esthétique et l'image d'une immense ressource encore mal connue, mais qu'il faut préserver. La sortie du film Paysage du Silence de Jacques-Yves Cousteau en 1947 est une étape importante de cette première prise de conscience. Avec les représentations contemporaines des paysages, les progrès technologiques permettent au plus grand nombre de découvrir le monde sous-marin. Les arts et la littérature ont consolidé cette relation en rendant hommage à sa beauté et à son mystère, à l'image du douzième album de bande dessinée des Aventures de Tintin, Le Trésor de Rackham le Rouge - paru en couleurs en 1945. Les peintres-plongeurs ou les artistes de la rue représentent de nos jours ces paysages toujours emprunts d'imaginaires, mais aussi porteurs curiosités insoupçonnées.

10 Cette courte histoire des représentations montre que la notion traverse le temps et que l'évolution des techniques renouvelle les regards et les images que l'on porte aux paysages sous-marins. Si la connaissance scientifique du paysage progresse, elle n'évacue pas sa dimension sensible, liée à l'expérience et à la sensibilité de tout être qui fabrique aussi son paysage et en définit ses valeurs. Ces valeurs peuvent être celles du beau ou du laid, du paysage artialisé 8 , d'espèces banales ou plus emblématiques que l'on souhaite protéger. Cette esthétisation participe aussi à la mise en scène du paysage et à sa diffusion par les médias; le paysage médiatisé est notamment celui qui évoque ces hauts lieux de nature et de biodiversité ou, à l'inverse, l'« océan poubelle » réceptacle de tous les déchets. 
11 Au travers des perceptions sensorielles conditionnées par le progrès des techniques et des représentations porteuses d'images et de valeurs, le paysage sous-marin navigue finalement entre deux pôles, le premier est du domaine des matérialités et rend le paysage objectivable ; le second est du registre des représentations et des inventions, le paysage est alors envisagé dans sa relation avec les hommes, il devient une construction sociale, culturelle, porteur d'émotions, de valeurs, et se prête à une forme de théatralisation, sorte de spectacle ou de comédie humaine, qui permet au paysage de se réinventer en permanence.

12 Afin de mettre à l'épreuve cette notion, nous avons choisi un cas d'étude appliqué à l'archéologie littorale. Nous montrons l'utilité d'une approche subaquatique pour enrichir les connaissances d'une structure archéologique située dans son continuum terre/mer. Après avoir présenté le site et posé quelques hypothèses quant à l'origine de cette structure et à son insertion géoenvironnementale, un ensemble de données est recueilli puis analysé. Le paysage devient un cadre, mais aussi un révélateur des dynamiques terrestre et marine et par là même un outil permettant d'améliorer la connaissance d'un site doté aussi d'une grande valeur patrimoniale.

\section{Analyser le paysage sous-marin : application à la géoarchéologie littorale}

\section{Un cas d'étude : la pointe de Porzh Karn en Bretagne (Penmarch, Finistère)}

\section{Présentation du site}

13 Afin d'étayer nos propos, nous avons choisi de les illustrer via l'étude d'un site énigmatique implanté en milieu intertidal au sein du pays bigouden, dans le sud du Finistère. La pointe de Porzh Karn (fig. 1), située à environ 2,5 kilomètres à vol d'oiseau du bourg de Penmarch, est une avancée rocheuse orientée nord-est faisant face à la pointe de la Torche (Plomeur) distante de 1,1 kilomètre. 
Fig. 1. - Localisation de la pointe de Porzh Karn.

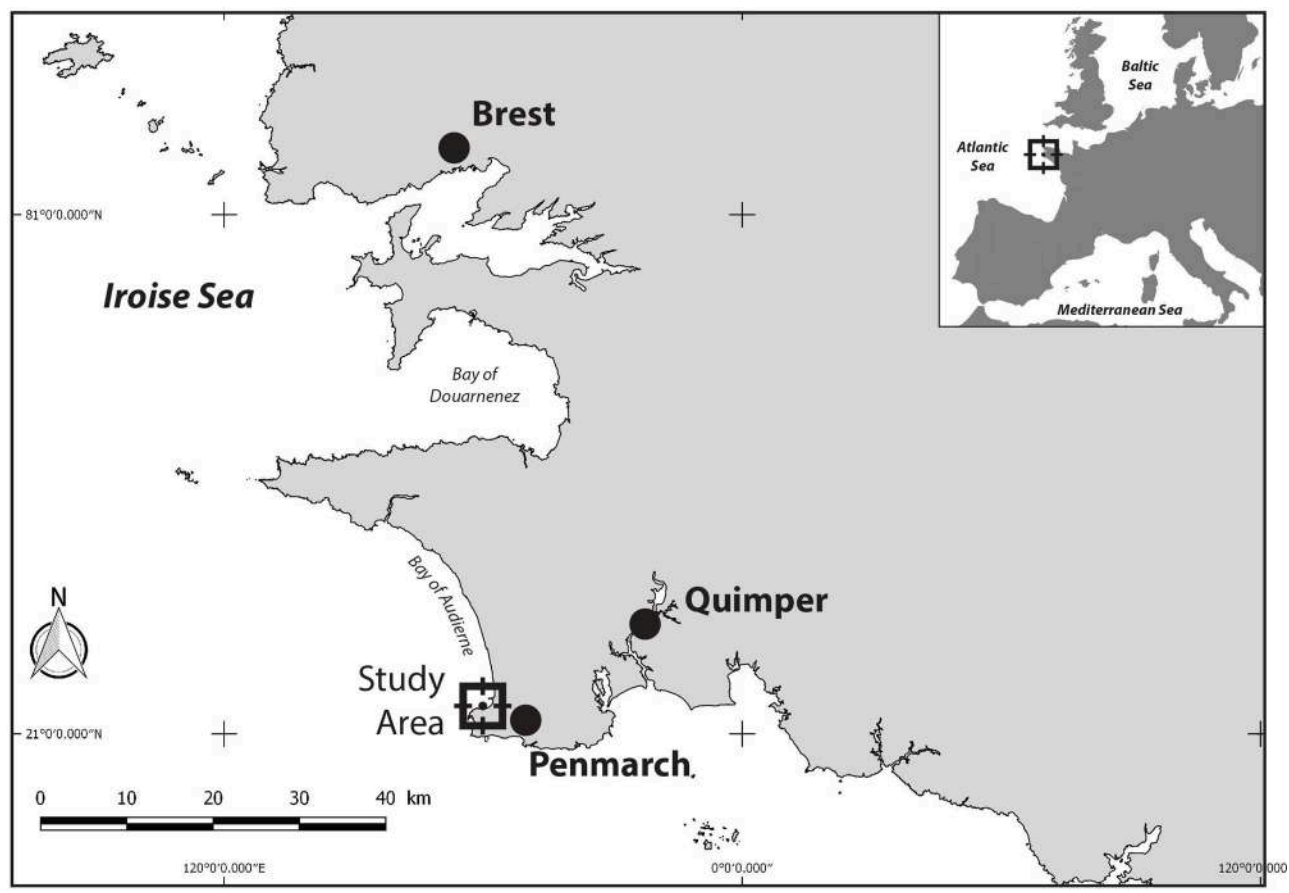

DAO Gaëtan Jolly.

Elle marque la transition entre le paysage dunaire de l'anse de Porzh Karn (massif dunaire de Toul Gwin) située à l'est et l'environnement granitique du trait de côte de Saint-Guénolé au sud-ouest. Ce territoire, battu par les éléments, possède une altimétrie inférieure à 10 mètres, et s'étend jusqu'à la pointe de Porzh Karn qui culmine à 6 mètres NGF (fig. 2).

Fig. 2. - Localisation des structures anthropiques de la pointe de Porzh Karn.

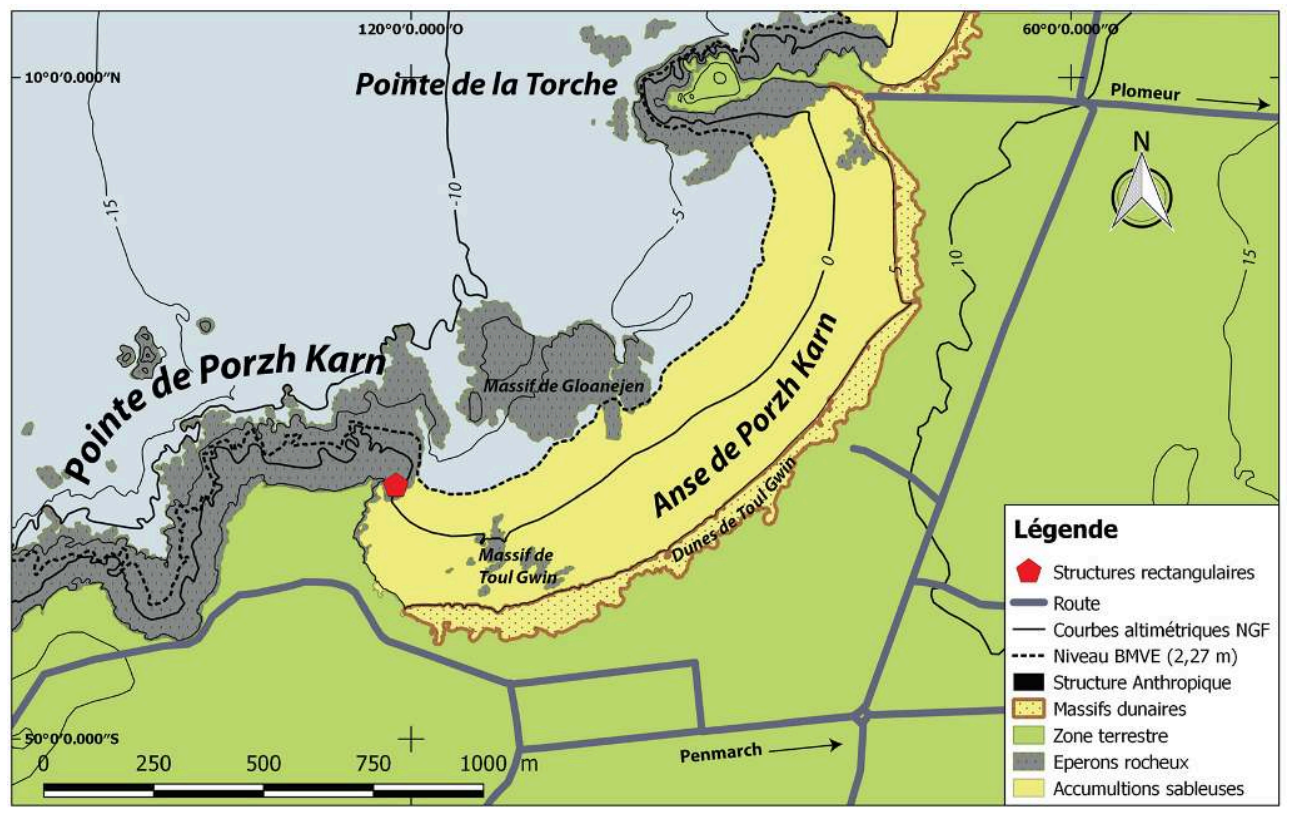

DAO Gaëtan Jolly. 
15 C'est sur l'estran en partie nord-est de la pointe que l'on observe sur les photographies aériennes des formes rectangulaires délimitées par des empierrements (fig. 3). L'ensemble du site couvre une surface au sol d'environ $2500 \mathrm{~m}^{2}$.

Fig. 3. - Plan masse de la structure anthropique de la pointe de Porzh Karn.

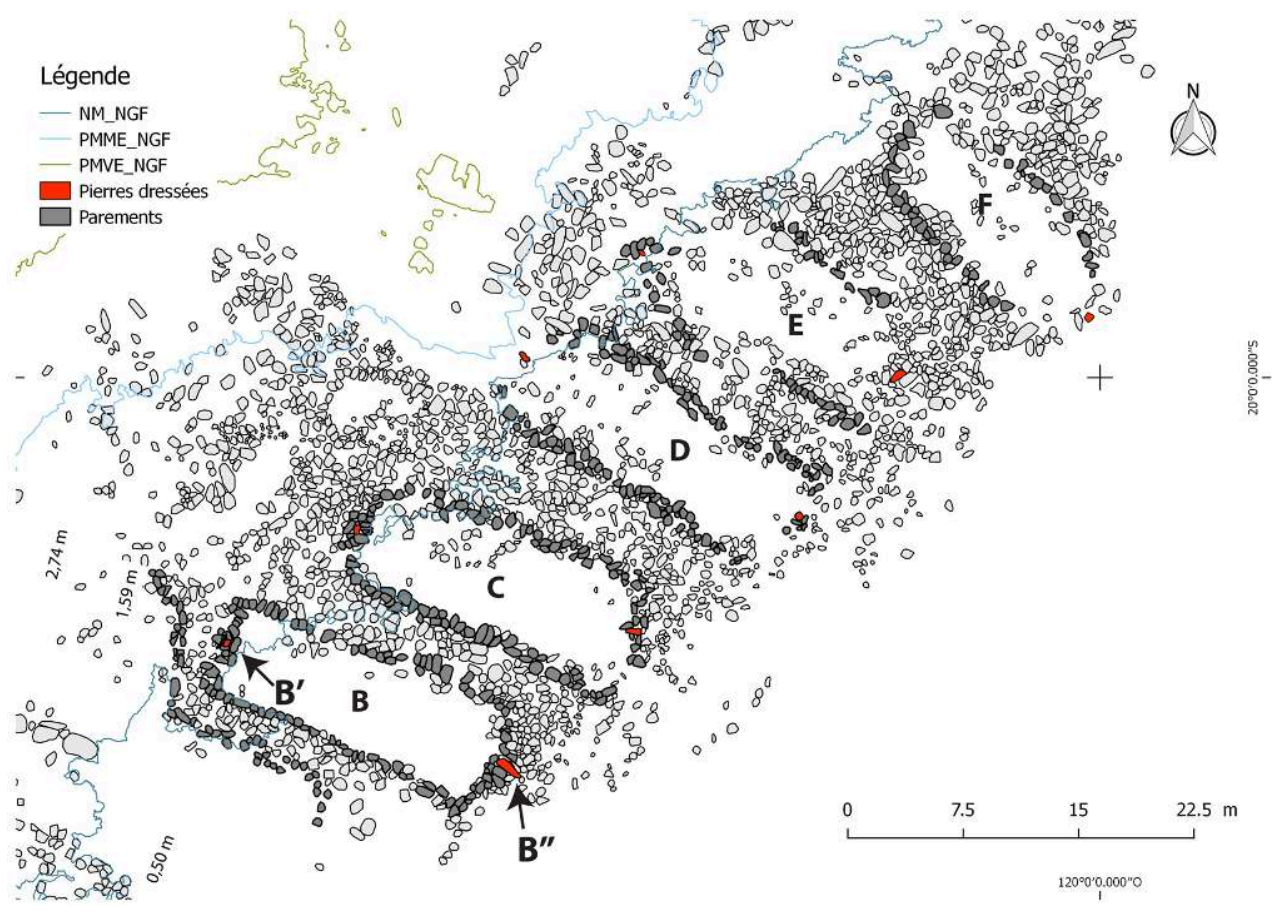

DAO Gaëtan Jolly.

\section{Brève description des structures}

Intrigué par ces structures, des recherches ont été amorcées afin de comprendre à quoi cet ouvrage pouvait servir. Cependant, aucun ouï-dire local ou élément bibliographique ne mentionnent des faits avérés. À la suite de relevés archéologiques, nous avons observé que ces structures rectangulaires forment un ensemble orienté ouest-nordouest - est-sud-est, abrité partiellement de la houle dominante.

Ces constructions ont la particularité de suivre l'inclinaison de la pente naturelle de l'estran et d'être disposées sur un axe nord-est - sud-ouest. Elles possèdent des dimensions quasi identiques les unes aux autres. Composées de blocs roulés décimétriques (jusqu'à $60-80 \mathrm{~cm}$ ), leurs longueurs avoisinent les 20 mètres pour des largeurs d'environ 5 mètres, soit une surface équivalente à $100 \mathrm{~m}^{2}$, constituées à chaque extrémité de pierres dressées atteignant en moyenne 1,20 mètre de hauteur, sur laquelle on distingue en partie supérieure une rainure (fig. 4). 
Fig. 4. - Pierre dressée rainurée sur le pourtour en partie haute.

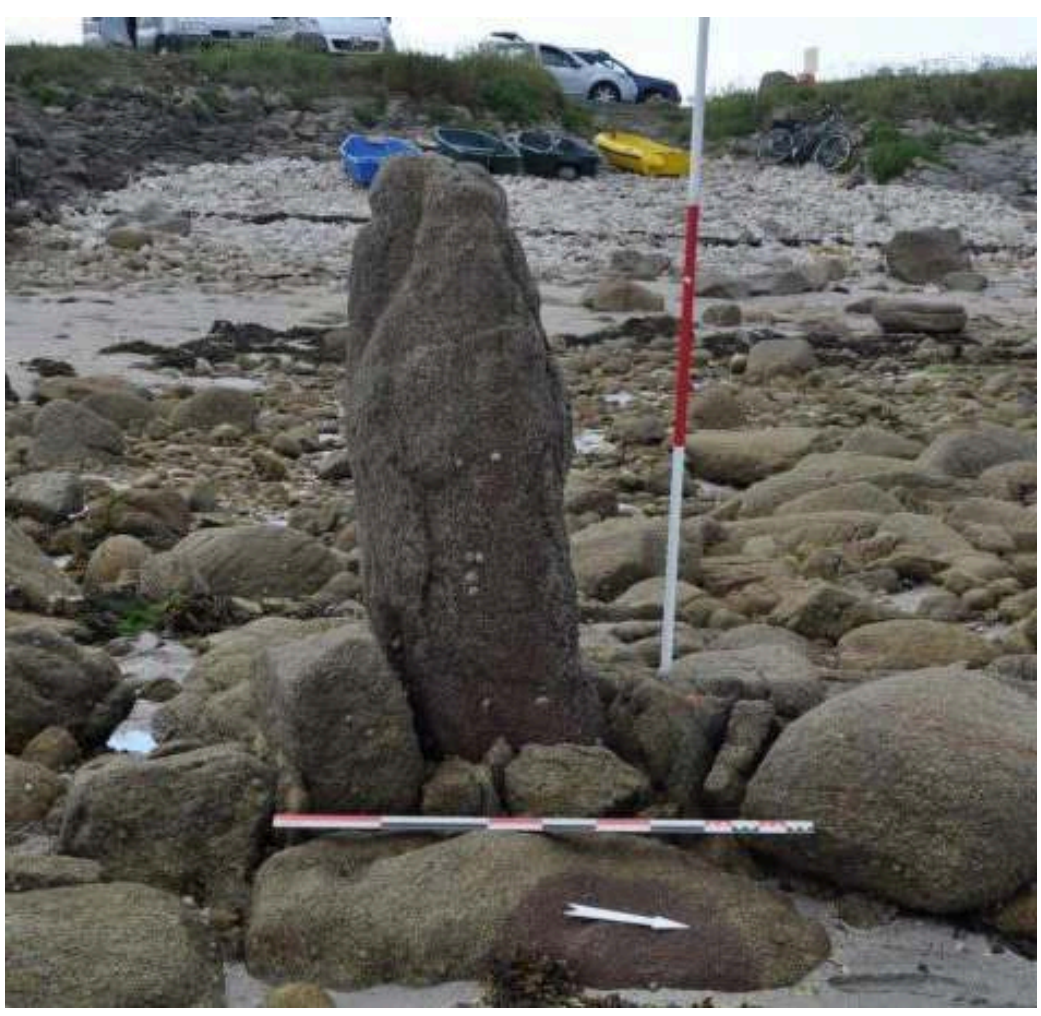

Photo Gaëtan Jolly @

\section{État de l'art} structures comme :

«S'agissant d'emplacement d'échouage de barques de pêche, la proue et la poupe étroitement maintenues par des cordes9."

Cependant, cet auteur n'apporte aucun élément de datation ni de référence bibliographique. Une analyse de la microtoponymie nautique par A. Le Berre ${ }^{10}$ fait mention de la dénomination locale Beg ar Pont (la pointe de la chaussée), ainsi que la traduction de Portz Carn (port du tas de pierres) faisant référence très certainement aux cairns néolithiques érigés à la pointe de Porzh Karn.

\section{La littérature anglo-scandinave : une piste éventuelle ?}

Aucune indication dans les archives n'a permis de repérer la présence d'un quelconque aménagement anthropique aux périodes modernes ou contemporaines. Il en est de même au bas Moyen Âge où aucun port n'est notifié dans les sources. D'un point de vue archéologique, il est possible de penser que cette structure présente des similitudes avec les ouvrages vikings construits sur l'estran pour abriter leurs navires ${ }^{11}$. La littérature anglo-saxonne décrit ces constructions sous le terme de boat shelters, boat houses, boat sheds («abri à bateau »), tout comme les termes nousts ou nausts d'origine scandinave $^{12}$. Ces abris permettaient d'accueillir des bateaux pour les périodes d'hivernage ou de maintenance ${ }^{13}$. Parallèlement, l'implantation des Vikings en 
Cornouailles est avérée entre la fin du $\mathrm{Ix}^{\mathrm{e}}$ et le début $\mathrm{du} \mathrm{x}^{\mathrm{e}}$ siècle ${ }^{14}$. Si l'on se réfère à cette période, le niveau marin pour le sud du Finistère est inférieur d'un mètre à l'actue ${ }^{15}$, ce qui rend le site protégé de la houle de suroit. Cela fait de cet espace un lieu propice au mouillage, contrairement à la côte environnante qui ne dispose pas d'abri naturel. Cette structure peut-elle être assimilée à un ancien port?

21 L'analyse du site a permis de mettre en évidence une lacune bibliographique importante qui laisse de nombreuses interrogations quant à la période d'édification et à l'utilité de ce vestige archéologique. Néanmoins, le bon état de conservation de l'ouvrage indique d'étroites similitudes avec les structures étudiées dans les régions anglo-scandinaves. Nous avons choisi de définir deux pistes de recherches. L'une s'oriente vers l'analyse approfondie des archives, appuyée par Julien Bachelier (docteur en histoire médiévale), dans l'espoir de contextualiser l'édification de l'ouvrage dans son histoire. La seconde piste repose sur l'hypothèse d'une infrastructure portuaire, en raison de la configuration particulière des lieux. En effet, si ce présent ouvrage fut conçu pour abriter des navires, cela signifie que le contexte géographique s'y prête. Cette seconde orientation nous a conduits à étudier les dynamiques physiques de ce milieu situé à l'interface entre l'espace terrestre et sous-marin, dans l'espoir de comprendre l'insertion de cette structure dans son environnement.

\section{Interprétation multiscalaire et retranscription des paysages}

L'objectif est d'enrichir les éléments de réponse sur l'histoire de cette structure dans son continuum terre/mer perçu par une approche interdisciplinaire. Pour ce faire, nous avons tablé sur l'élaboration d'une analyse géographique regroupant l'ensemble des dynamiques physiques perçues sur ce territoire par le prisme de l'interprétation paysagère. Le but est d'obtenir une lecture globale des interactions biogéophysiques présentes sur le site pour établir une grille d'observation qui associe la description, la visualisation et l'explication des formes du relief terrestre et sous-marin, puis les relations entre l'homme et son milieu ${ }^{16}$. D'un point de vue archéologique, ce travail géographique du site permettra d'étayer ou de réfuter l'hypothèse d'une infrastructure portuaire sur la pointe de Porzh Karn. Bien entendu, l'analyse des données existantes, telles que les relevés lidars, met en évidence la configuration physique des fonds marins. Néanmoins, l'interprétation d'une carte bathymétrique ne suffit pas à établir des conclusions sur le contexte sous-marin du site. L'évolution technologique permet d'aboutir à une meilleure appréciation des fonds marins, mais des prospections ponctuelles sur le milieu ne sont pas à exclure. En effet, ces dernières permettent d'enrichir nos contenus, en plus d'apporter une dimension sensorielle aux dynamiques marines.

L'objectif est d'appréhender notre site à travers une interprétation multiscalaire ${ }^{17}$. Pour ce faire, il est nécessaire de considérer trois niveaux scalaires d'interprétation paysagère: le niveau des composantes paysagères $(<10 \mathrm{~m})$, l'unité paysagère ou géofaciès $(10-100 \mathrm{~m})$ et le niveau du géosystème $(>100 \mathrm{~m})^{18}$. Cette collecte mise bout à bout laisse entrevoir, in fine, une dimension paysagère des fonds marins. La finalité est de délimiter et de caractériser un paysage en croisant les méthodes d'analyse, objectives et subjectives, en parallèle d'une pluridisciplinarité des méthodes d'approche, à pied ou en plongée. 


\section{Exploitation des données existantes} dont $29000 \mathrm{~m}^{2}$ empiètent sur la zone d'étude puis au sud-ouest par la pointe de Porzh Karn avec un couvert étudié de $35000 \mathrm{~m}^{2}$. C'est une étendue sableuse de 77,2 hectares ponctuée par deux massifs rocheux découverts en leurs extrémités par les marées basses de morte-eau : au centre le massif de Gloanejen (93000 m²), à l'extrême sud-est le massif de Toul Gwin $\left(10000 \mathrm{~m}^{2}\right)$. Un couvert végétal composé en majorité de laminaires (Laminaria sp.) et de fucales (Fucus sp.) recouvre les récifs dans leurs parties submergées. Descendant en pente douce, le dénivelé y est très faible avec une profondeur maximale de -10 mètres (cote NGF) (fig. 5).

Fig. 5. - Cartographie de l'orientation de la houle dominante OSO.

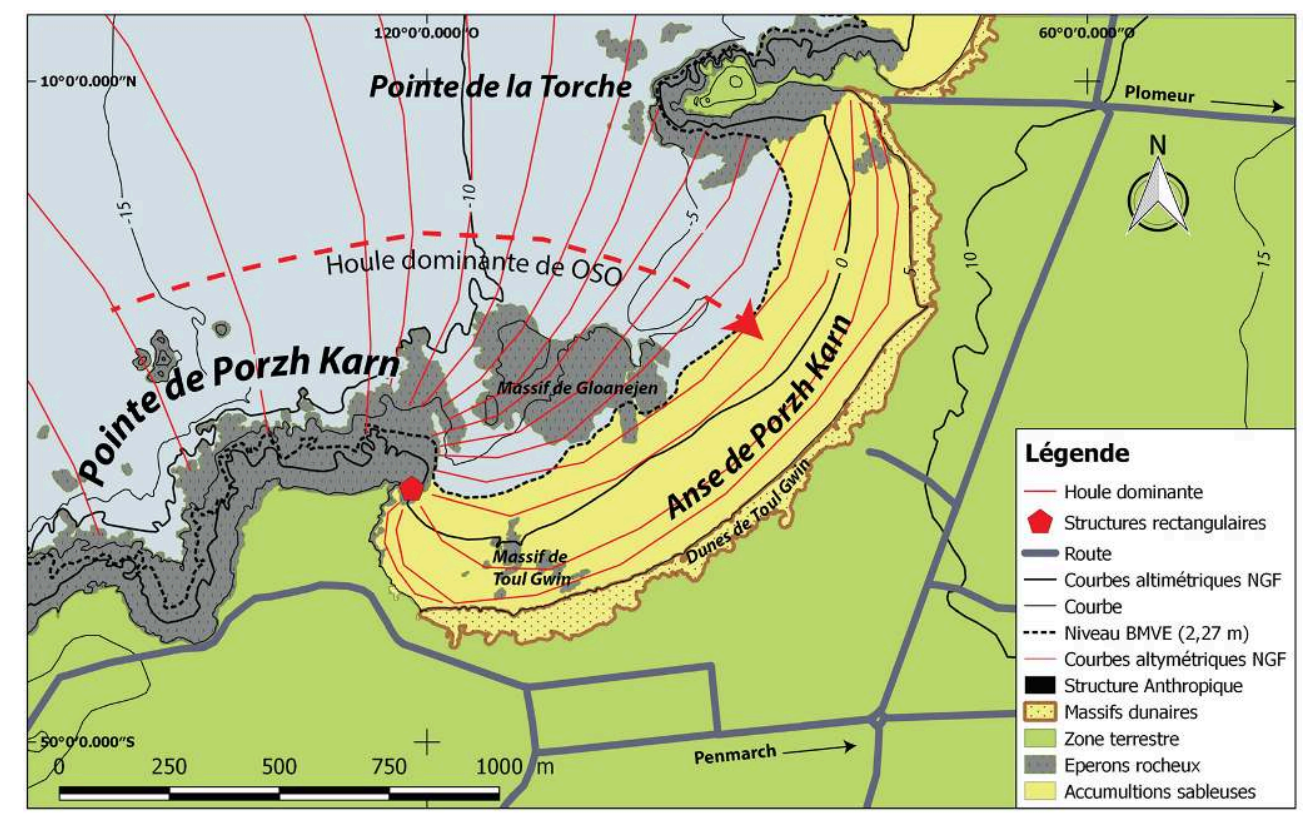

DAO Gaëtan Jolly.

\section{Plan de plongée et méthode de prospection}

L'orientation en plongée est une des difficultés propres à cette pratique. Par conséquent, nous avons conçu un plan de plongée en nous appuyant sur les données bathymétriques Litto3D de 2014. Un quadrillage de la zone est réalisé pour définir des caps à suivre afin d'accomplir une couverture d'observation. L'objectif est de proposer un maillage de données, en partant d'une analyse limitée à l'échelle d'un élément paysager pour aboutir à un assemblage d'observations. La finalité est d'élargir ces interprétations à l'échelle du territoire de l'anse.

Pour réaliser nos observations sous-marines, nous nous sommes inspirés des méthodes de prospections archéologiques et de sauvetages subaquatiques; ces méthodes sont 
basées sur le facteur de turbidité, et ont pour avantage d'être quantifiables. Plusieurs méthodes existent à ce sujet, l'une d'entre elles nous paraît la plus appropriée, car elle peut être réalisée par un seul observateur. Cette méthode est appelée « circulaire » ou encore « technique des cercles complets ${ }^{19}$ ".

Néanmoins, quelques modifications ont été apportées afin d'accentuer la précision des mesures de distance. Pour appliquer cette méthode, le matériel nécessaire est un jalon, une corde, une bouée signalétique, un grappin pour la mise en station et un GPS (fig. 6).

Fig. 6. - Schéma de prospection en circulaire.
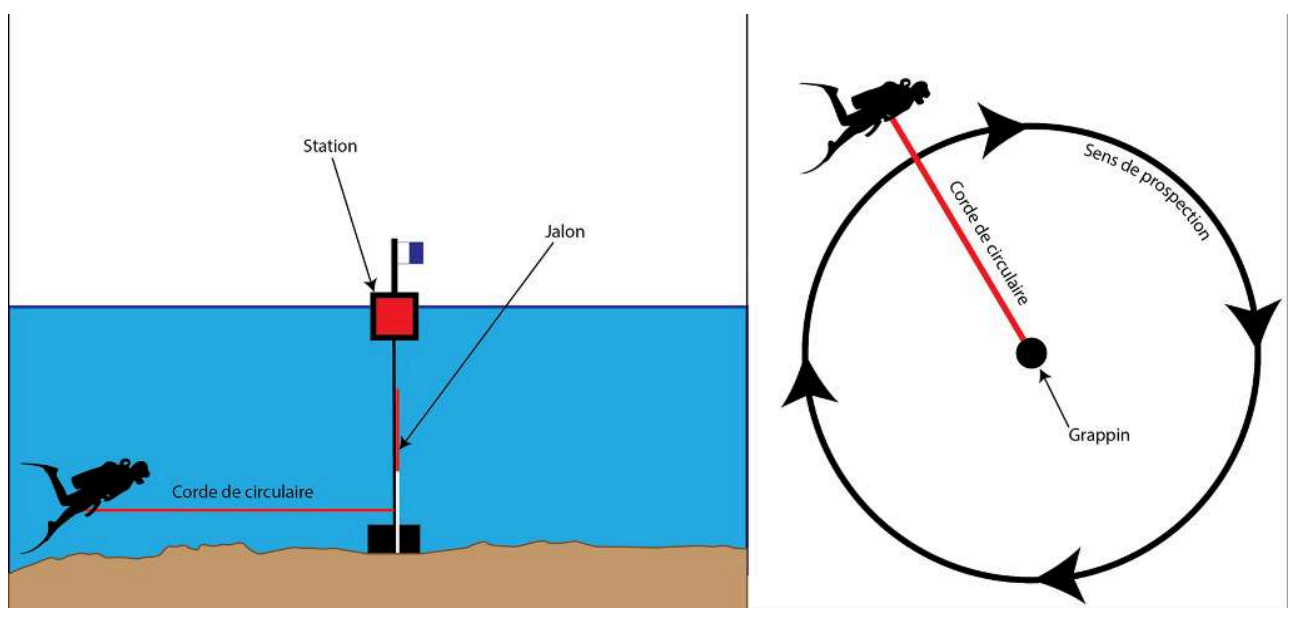

DAO Gaëtan Jolly.

Le plongeur, une fois arrivé sur le lieu de prospection, établit la mise en station. L'opération consiste à mouiller un grappin et à y arrimer la bouée signalétique en surface. Sous l'eau, le plongeur dispose un jalon en vertical au pied du grappin. Celui-ci sert de point visuel, observable facilement dans l'eau. Le plongeur fixe une corde au pied du jalon, puis la déroule jusqu'à la limite de distinction du jalon. Cet écart visuel entre le jalon et le plongeur définit la distance visuelle de la circulaire. Ensuite, il nage en cercle autour de la station, ce qui lui permet de réaliser une analyse des composantes paysagères environnantes, tout en ayant un point de repère en soutien. Ainsi, l'écart de chaque mise en station est défini selon la distance de visibilité du rayon d'action. Cette méthode permet de géoréférencer chaque mise en station puis de les assembler afin d'obtenir une couverture du site cartographiable, comparable à une prospection aérienne.

\section{Méthode de retranscription}

Étant sous l'eau et en apnée, la méthode de retranscription doit elle aussi être anticipée. Ainsi, une grille d'analyse pré-remplie est inscrite sur une planche submersible. Elle comporte les différentes notions paysagères, inspirée des critères d'observations sous-marines des biologistes et géomorphologues. Les termes utilisés ont été retranscrits dans une fiche descriptive, qui se divise en cinq parties :

- La première est basée sur l'objectivité et a pour but de définir les caractéristiques physiques des fonds. Elle se découpe en quatre critères d'analyse: le substrat, la topographie, la géomorphologie et les facteurs météorologiques. Cette première étape permet de recontextualiser la partie subjective dans son contexte météorologique ; 
- La deuxième est basée sur les facteurs biologiques permettant de décrire les zones recouvertes ou non par la végétation, et d'entrevoir les populations faunistiques pouvant donner des indices sur la dynamique des courants. Elle se découpe en deux parties : l'indice floristique et faunistique ;

- La troisième est basée sur la subjectivité permet d'exprimer les sensations ressenties lors de la plongée, telle que les zones exposées au courant et celles plutôt abritées. Ces observations permettent d'enrichir la description géographique du lieu en décrivant le comportement des facteurs physiques sur le site ;

- La quatrième a pour objectif de synthétiser la caractéristique paysagère de la station ;

- Enfin, un espace vide est laissé, dans l'optique de réaliser une représentation schématique du site par une synthèse globale des observations relevées.

31 Cette méthode, malgré son aspect subjectif, nous a permis de couvrir une grande partie de l'anse (fig. 7). L'ensemble des données recueillies est synthétisé via une représentation graphique et textuelle paysagère présente en troisième partie.

Fig. 7. - Carte des prospections réalisées relevant les mises en station et les unités paysagères.

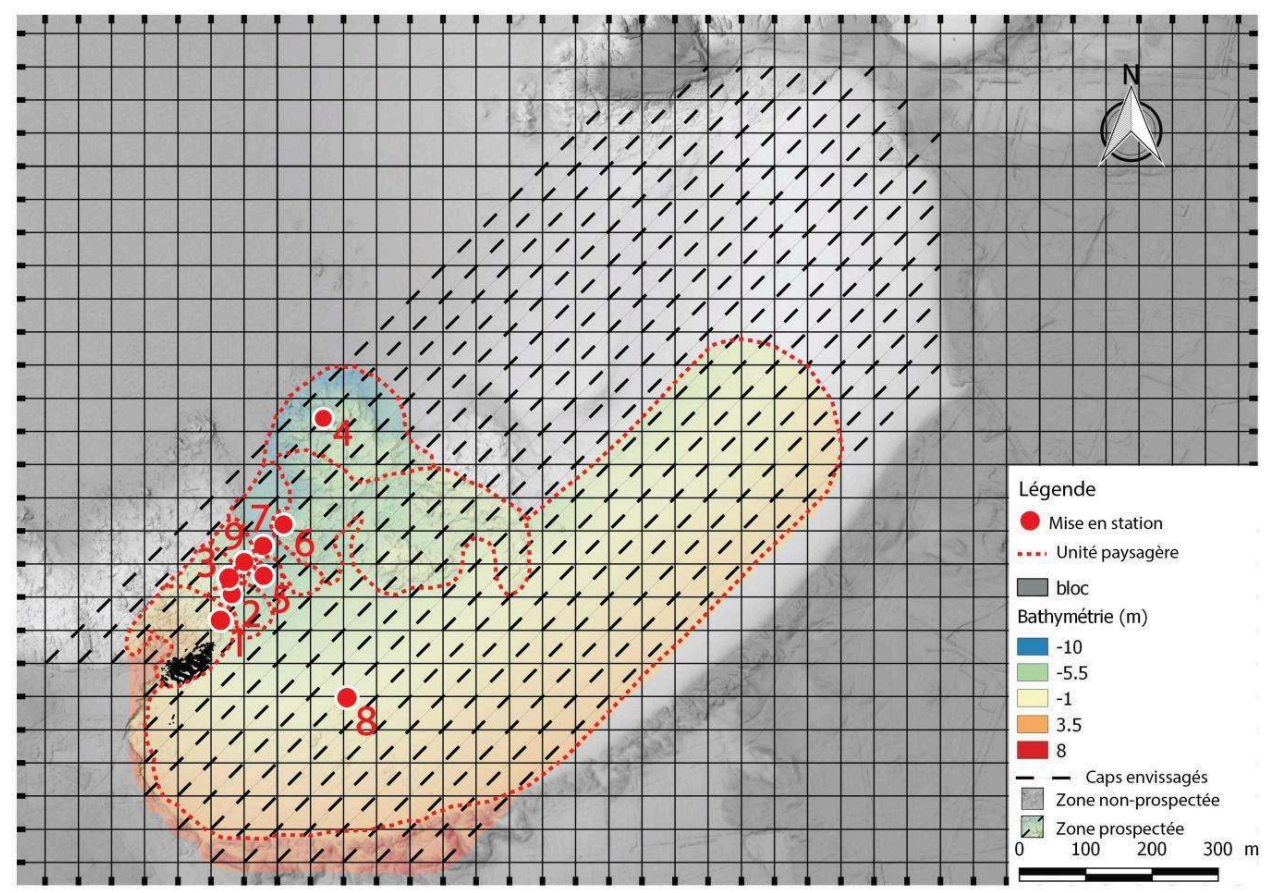

DAO Gaëtan Jolly.

\section{Analyse sous-marine et gestion patrimoniale des zones côtières}

Les relevés archéologiques et paysagers ont permis d'appréhender les méthodes de construction employées sur le site et d'interpréter l'implantation de cet ouvrage dans son contexte géographique. Analysés à différents niveaux, les résultats ont permis de démontrer qu'à l'échelle du site les structures sont similaires entre elles et qu'elles sont prolongées par un aménagement maçonné en haut d'estran. À l'échelon du paysage, 
l'implantation d'une infrastructure portuaire peut être justifiée par un contexte sousmarin favorable.

\section{L'interprétation terrestre}

L'analyse paysagère (fig. 8) met en évidence la platitude de la région. Ce manque de relief rend difficile la lecture du territoire pour un observateur néophyte venant $d u$ large. Rares sont les signes visuels distinctifs qui permettent de se situer le long du trait de côte. Au nord, la baie d'Audierne forme une étendue sableuse d'une trentaine de kilomètres au relief plat et uniforme. Au sud, l'éperon rocheux de Saint-Guénolé s'étend sur une quinzaine de kilomètres et offre un paysage minéral qui n'apporte que très peu d'éléments visuels distinctifs. Toutefois, au milieu se trouve l'anse de Porzh Karn qui forme une rupture entre ces deux contextes paysagers, ce qui la rend facilement observable depuis le large. Cette anse marque la transition entre un espace dunaire et minéral pris en étau par deux pointes rocheuses. Au nord, la pointe de la Torche qui culmine à 17 mètres indique une transition paysagère qui laisse apparaître au sud une étendue de sable s'étalant sur 1,5 kilomètre avant de rejoindre la pointe de Porzh Karn. Cette configuration géographique permet de créer une rupture visuelle facilement reconnaissable pour les navigateurs.

Fig. 8. - Bloc-diagramme de l'anse de Porzh Karn.

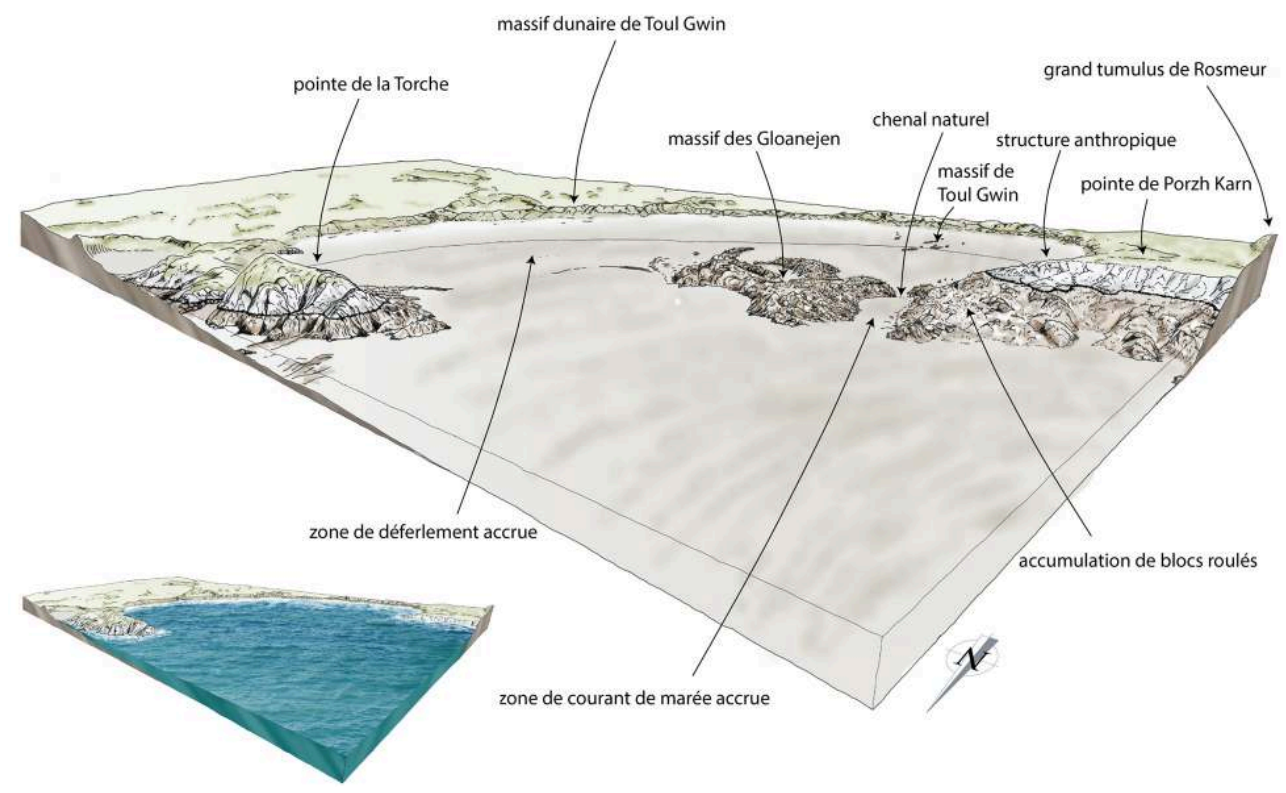

DAO Gaëtan Jolly.

Autre point paysager important, la forme de la végétation et du relief indique un fort impact des éléments et plus précisément du vent sur ce territoire. Les six mètres d'altitude de la pointe de Porzh Karn permettent d'atténuer le souffle du vent dominant venu d'ouest. Situé à quatre mètres plus bas, l'estran exposé sur la façade sud-est de la pointe s'avère être un abri naturel offrant un accès protégé à l'espace littoral. De plus, nos observations montrent que de manière générale la houle est beaucoup plus prononcée au nord-est de l'anse qu'au sud-ouest, faisant de cet endroit une zone d'eau calme idéale pour le mouillage de bateaux. 


\section{L'interprétation sous-marine} primordial pour une activité portuaire militaire ou commerciale. Ce lieu offre un abri cerné de défenses naturelles. L'environnement subtidal se compose de hauts-fonds faisant face à de fortes houles. Ces conditions rendent ce lieu accessible à des navigateurs initiés disposant de bateaux spécifiques à faible tirant d'eau leur permettant de s'échouer sur le sable. Le site possédait également la particularité d'être facilement identifiable au large par les navigateurs. En effet, en plus du contexte

Parallèlement, la prospection sous-marine nous a permis d'avoir une image concrète de la configuration géographique du milieu subaquatique et de percevoir l'interaction des dynamiques physiques qui s'y produisent (fig. 8).

roulés à la granulométrie hétérogène, allant du galet aux blocs d'un mètre de diamètre, étalés sur une distance de 350 mètres allant jusqu'à 8 mètres de profondeur, orientés est-nord-est. Cette observation souligne une désagrégation de la roche mère granitique de la pointe. Ce qui laisse à penser que l'étendue rocheuse de Porzh Karn se prolongeait quelques centaines de mètres plus loin dans l'anse. Toujours sous l'eau, une fois cette accumulation dépassée vers le nord-est, le massif rocheux laisse place à un banc de sable ininterrompu de 25 mètres de long sous 8 mètres de profondeur. Celui-ci ne possède aucune couverture végétale et s'étale jusqu'à atteindre le récif des Gloanejen situé au nord. Ce banc de sable cerné par les deux éperons rocheux forme un chenal naturel qui est emprunté par les canots de pêche. Ce contexte forme un entonnoir qui accentue la force du courant de marée, et qui donc complexifie la navigation. La marée montante permet aux bateaux de rentrer mouiller au sud-est de la pointe de Porzh Karn. À l'inverse, la marée descendante permet aux navires de gagner le large. laminaires, il fait directement face à la houle entrant dans l'anse. Il forme avec la pointe de Porzh Karn un brise-lames naturel réduisant la houle dominante. Cette configuration engendre une perte de puissance de la houle qui est orientée ouest-sudouest. Par conséquent, celle-ci est déviée par un phénomène de diffraction et vient finir sa course au sud-ouest de l'anse. Ainsi, la houle dominante a très peu d'impact sur le site archéologique et les mouillages des bateaux de pêche.

\section{L'hypothèse du port à bateau confortée par les données paysagères}

La combinaison des interprétations paysagères terrestres et sous-marines offre une synthèse continue du contexte paysager du sud de l'anse de Porzh Karn. Ainsi, l'analyse paysagère fait valoir le caractère stratégique de l'anse de Porzh Karn pour y implanter un édifice portuaire. Si cette fonctionnalité est avérée, l'étude met en évidence la manière dont l'homme a su tirer profit de l'environnement. Cela laisse à penser que le site a bien été construit afin de ne pas être impacté par la houle et le vent. En effet, les structures s'appuient sur la roche mère en bas d'estran afin d'être abritées des vents dominants. Puis les abris ont sans doute été construits de façon opportuniste en utilisant les blocs rocheux disponibles sur l'estran.

D'un point de vue stratégique, la configuration de la pointe de Porzh Karn est un atout 
paysager particulier, il y avait la présence de deux tumulus, dont celui de Rosmeur qui a été détruit à la fin du XIXe siècle. Le tumulus apportait un support visuel, tel un amer, perceptible facilement avec ces six mètres de hauteur et quarante mètres d'envergure $^{20}$. De plus, exposé au sud-est, le site est rendu invisible du large, les bateaux pouvaient être facilement masqués.

\section{Parvenir par la connaissance à une meilleure reconnaissance des paysages sous-marins}

40 Face à un site énigmatique qui ne présente aucune trace historique ou archéologique antérieure, l'interdisciplinarité s'avère être une solution permettant d'élargir les champs de recherches facilitant ainsi la compréhension du site.

41 Cet essai de protocole d'analyse paysagère sous-marine nous permet d'appréhender un nouvel aspect de recherche de l'archéologie en zone intertidale et subtidale. Au premier abord, le lien entre l'archéologie et la notion du paysage sous-marin ne semble pas évident. Cependant, comme le montre notre cas d'étude, l'analyse subaquatique permet d'élargir les observations d'un pan de l'archéologie soumis au manque d'informations historiques et contraint par la hausse du niveau marin.

Il est vrai que les vestiges présents sur l'estran ont la particularité d'être constamment exposés aux éléments et par conséquent soumis à une détérioration accrue. Par conséquent, en s'inspirant de l'archéologie du paysage, l'analyse paysagère sousmarine pourrait apporter un nouvel élément d'interprétation facilement transposable. Elle pourrait aussi s'avérer déterminante pour l'analyse archéologique des anciennes populations côtières et préciser l'identification des paléopaysages dans le contexte du changement climatique.

Parallèlement, l'archéologie pourrait être un axe d'étude permettant de justifier l'utilité d'une approche paysagère sous-marine. En effet, celle-ci n'étant toujours pas reconnue par la Convention Européenne du Paysage, aucune politique n'est menée pour crédibiliser cette notion. Or, les différents travaux réalisés jusqu'à présent et les réflexions portées dans la sphère scientifique montrent l'intérêt à son égard. Ainsi, lier le paysage sous-marin à l'archéologie justifierait l'utilité de cette notion dans la gestion patrimoniale et l'élaboration de plans de gestion développés par les politiques de gestion intégrée des zones côtières (GIZC). Cette approche pourrait permettre d'élargir le champ des possibles et d'accentuer l'approbation de cette thématique dans la sphère politique en prouvant son utilité dans la sauvegarde et la gestion de l'environnement marin. Elle participerait à une nouvelle forme de territorialisation de la frange sousmarine du littoral ${ }^{21}$ et constituerait un nouvel outil de gestion de la frange littorale et subaquatique. 


\section{BIBLIOGRAPHIE}

ALLEN Anne 1995, «The maritime cultural landscape of Viking and Late Norse Orkney », thèse de doctorat en archéologie, Université de Durham, Durham.

AUGER Barbara 2011, «La représentation des bateaux en Europe entre le VIII et le XIII ${ }^{\mathrm{e}}$ siècle », thèse de doctorat en histoire de l'art, Grenoble, Université de Grenoble.

BADOUX Maurice et BINKERT Heinz 2007, « Recherches sous-marines », dossier technique, SISL, Genève, société internationale de sauvetage du Léman, 8 p.

Du Chatellier Paul 1879, « Les deux tumulus de Rosmeur, pointe de Penmarc'h (Finistère) », Matériaux pour l'histoire primitive et naturelle de l'Homme, $\mathrm{n}^{\circ} 10$ (2), p. 145-154.

CHEVALLIER Raymond 1976, « Le paysage palimpseste de l'histoire : pour une archéologie du paysage », Mélanges Casa Velázquez, nº 12 (1), p. 503-510.

DEHEUL Tiphaine 2016, « Les paysages sous-marins. Les derniers “nouveaux paysages", mémoire de Master 2, Théories et Démarches du Projet de Paysage », 115 p.

GARCIA-ARTOla Ane, STEPHAN Pierre, CEARRETA Alejandro, KopP Robert, KHAN Nicole, HORTON Benjamin 2018, « Holocene sea-level database from the Atlantic coast of Europe ", Quaternary Science Reviews, $\mathrm{n}^{\circ} 196$, p. 177-192.

GERVOIS Virginie 2014, «Étude sur les paysages marins et sous-marins dans le Parc naturel marin d'Iroise. Une expérimentation pour rendre visible ce qui se cache sous la surface ", Parc naturel marin d'Iroise, $25 \mathrm{p}$.

GIOT Pierre-Roland 1985, « Les dunes de la Baie d'Audierne », Bulletin de la Société Archéologique du Finistère, $\mathrm{n}^{\circ}$ 144, p. 9-14.

HALLAIR Gaëlle 2013, « Les carnets de terrain du géographe français Emmanuel de Martonne (1873-1955) : méthode géographique, circulation des savoirs et processus de visualisation », Belgeo, n 2, p. 5-25.

KERNALEGENN Tudi 2013, « Quand la Bretagne était viking », Ar Men, p. 12-19.

Le BERRE Alain 1961, Toponymie nautique de la côte sud du Finistère, Paris, Imprimerie Nationale, 99 p. MUSARD Olivier, FOURNIER Jérôme, MARCHAND Jean-Pierre 2007, « Le proche espace sous-marin : essai sur la notion de paysage », Espace Géographique, nº 36 (2), p. 168-185.

MUSARD Olivier et al., 2014, Underwater seascapes, from geographical to ecological perspectives, London, Springer.

MYRHE Bjørn 1985, « Boathouses as indicators of political organization, Norwegian Archeological Review, $\mathrm{n}^{\circ} 18$ (1), p. 36-60.

PETIT-BERGHEM Yves 2006, «L'apport des cartes et plans anciens dans la perception et la représentation du littoral de la Basse-Normandie », Revue du Comité Français de Cartographie, 168, p. $30-40$.

ROGER Alain 1997, Court traité du paysage, Mayenne, Éditions Gallimard, 199 p.

Roux Michel 1997, L'imaginaire marin des Français : mythe et géographie de la mer, Paris, Éditions L'Harmattan, $219 \mathrm{p}$. 
ZURCHER Frédéric et MARGOLLE Elie-Philippe 1868, Le monde sous-marin, Paris, Éditions J. Hetzel, 288 p.

\section{NOTES}

1. Deheul 2016, p. 35-45.

2. Musard et al. 2014, p. 85-155.

3. Ensemble des communautés végétales et animales d'un milieu donné.

4. Petit-Berghem 2006, p. 30-31.

5. Gervois 2014, p. 5.

6. Zurcher et Margolle 1868, p. 188-189.

7. Roux 1997, p. 10-11.

8. Roger 1997, p. 10-15.

9. Giot 1985, p. 9.

10. Le Berre 1961, p. 61.

11. Myhre 1985, p. 37.

12. Allen 1995, p. 57-58.

13. Auger 2011, p. 52.

14. Kernalegenn 2013, p. 2-6.

15. Garcia-Artola et al. 2018, p. 188.

16. Hallair 2013, p. 4-5.

17. Chevallier 1976, p. 504.

18. Musard et al. 2007, p. 176.

19. Badoux et Binkert 2007, p. 2-3.

20. Du Chatellier 1879, p. 145.

21. Musard et al. 2007, p. 169.

\section{RÉSUMÉS}

Le milieu sous-marin n'est exploré que depuis très récemment et la question de son paysage est une notion qui fait encore débat. Le paysage sous-marin soulève des enjeux tenant à son accessibilité et à sa mise en valeur. Il interpelle le scientifique soucieux d'étudier un sujet qu'il connaît mal et présente un intérêt pour le citoyen, pour peu qu'il fasse l'objet d'une appropriation tenant compte de la vision des différents acteurs des territoires maritimes. Parvenir à mieux connaître ces paysages sous-marins passe donc par un travail collaboratif et citoyen. Le chercheur doit s'appuyer sur des collaborations multi-niveaux et transdisciplinaires. L'archéologie peut s'avérer un axe d'étude permettant de justifier l'utilité d'une approche paysagère sous-marine. En effet, au-delà de l'intérêt archéologique, l'étude paysagère des 
vestiges engloutis est un moyen de sensibiliser le grand public au domaine sous-marin et, plus spécifiquement, de déclencher une démarche de réappropriation d'un patrimoine oublié ou en voie de disparition.

\section{AUTEURS}

\section{YVES PETIT-BERGHEM}

Géographe, professeur en écologie, École nationale supérieure de paysage (ENSP), Versailles, membre du Laboratoire de recherche en projet de paysage (LAREP/ministère de l'Agriculture)

\section{GAËTAN JOLLY}

Doctorant, Institut universitaire européen de la mer (IUEM), LETG (Littoral-EnvironnementTélédétection-Géomatique) UMR 6554, Technopôle Brest-Iroise 
Imaginer l'aménagement 


\title{
Des sources pour l'histoire de l'aménagement du territoire : les archives du tunnel sous la Manche conservées aux Archives nationales
}

\author{
Stéphane Rodriguez-Spolti
}

1 Les Archives nationales ont :

«Pour mission de collecter, classer, inventorier, conserver, restaurer, communiquer et mettre en valeur les archives publiques provenant des administrations centrales de l'État, les archives des notaires de Paris et des fonds privés d'intérêt national ${ }^{1}$. »

De ce fait, sont conservées aux Archives nationales les archives relatives au tunnel sous la Manche produites ou reçues par le ministère des Travaux publics, le ministère des Transports et les organismes qui leur ont été rattachés durant toute l'histoire de ce projet d'aménagement. Il s'agit donc principalement des dossiers du contrôle du ministère des Travaux publics et des Transports sur les projets successifs présentés depuis les années 1860 .

3 L'ensemble du fonds d'archives relatives au tunnel sous la Manche représente plus d'une centaine de mètres linéaires de documents versés aux Archives nationales durant près d'un siècle, le dernier versement ayant eu lieu en 2016. Il couvre la période 1867-2008 et se répartit entre la sous-série $\mathrm{F} / 14^{2}$ relative aux travaux publics et une succession de versements conservés en cotation continue ${ }^{3}$.

4 Ces archives sont constituées des dossiers d'enquêtes publiques, de consultations et des projets proposés, des dossiers d'études techniques et financières, des relations intergouvernementales franco-britanniques et d'archives figurées telles que des cartes, des plans, des photographies ainsi que quelques maquettes. L'ensemble de ces archives est consultable aux Archives nationales, en salle de lecture à Pierrefitte-sur-Seine.

Le chercheur pourra ainsi découvrir les fonds de cabinets ministériels ${ }^{4}$ dont, en particulier, les dossiers de Jean-Pierre Ghuysen, chargé de mission auprès de Jean- 
Pierre Douffiagues, ministre des Transports. Inspecteur général des transports et des travaux publics, Jean-Pierre Ghuysen a été président de la délégation intergouvernementale au tunnel sous la Manche, puis, depuis 1985, successivement secrétaire général auprès de la commission franco-britannique, secrétaire général du tunnel, puis président de la commission intergouvernementale du tunnel sous la Manche. Impliqué depuis près de deux décennies dans le projet, les archives produites par M. Ghuysen, versées en 1989 aux Archives nationales sous la cote 19890272, représentent ainsi une des principales sources pour l'histoire récente du suivi du projet du tunnel sous la Manche.

\section{Cent mètres linéaires d'archives pour un siècle et demi d'histoire}

En 1977, sont versés aux Archives nationales les fonds des archives produites par la délégation au tunnel sous la Manche, la Société française du tunnel sous la Manche $(\mathrm{SFTM})^{5}$ et la Société d'Ingénierie du Tunnel sous la Manche (SITUMER), ainsi que la division du tunnel sous la Manche. Ces versements offrent aujourd'hui aux chercheurs la possibilité de prendre connaissance des relations franco-britanniques pour mener à bien le projet lancé dans les années 1970.

7 Enfin, en 2008 et 2016, sont successivement versées les archives produites par le Secrétariat général de la commission intergouvernementale du tunnel sous la Manche (1984-1997) ${ }^{6}$ et le Secrétariat général au tunnel sous la Manche (1985-2008). Au sein de la Direction générale des Infrastructures, des Transports et de la Mer (DIGTM), rattachée au ministère de la Transition écologique et solidaire, le secrétariat général au tunnel sous la Manche fait partie de la Direction des Services de transports (DST). Il assiste la délégation française à la commission intergouvernementale créée par l'article 10 du traité de Cantorbéry?.

Ces fonds d'archives couvrent, par conséquent, l'ensemble de la période concernée par les projets de franchissement de la Manche. Après cette rapide présentation des fonds et de leurs producteurs, nous vous proposons de découvrir les projets successifs, parfois étranges, étudiés, proposés et, pour certains, mis en œuvre pour traverser en sécurité la mer entre la France et la Grande-Bretagne.

De l'étude des archives conservées aux Archives nationales, plusieurs périodes successives dans les projets de franchissements de la Manche peuvent être identifiées.

\section{Les premiers projets (1867-1888)}

La première concerne les premiers projets sérieux et ambitieux qui voient le jour de 1867 à 1888. En 1867, à l'exposition universelle de Paris, Louis-Joseph Aimé Thomé de Gamond (1807-1876) présente son septième projet. Ingénieur des mines et ingénieur hydrographe, il réfléchit au projet de franchir la Manche depuis 1834. Il mène des études précises du fond de la Manche qui lui permettent de présenter un projet de tunnel avec, en son milieu, une île artificielle, l'île de Varne (fig.1). Cette étape permettait aux voyageurs de respirer un air frais, de changer de train, mais aussi de s'embarquer à bord de navires de compagnies maritimes à destination du monde entier grâce aux débarcadères prévus à cet effet. En 1869, il transmet au ministère des 
Travaux publics et des Transports plusieurs mémoires comportant ses études ainsi que des plans précis du sous-sol entre la France et la Grande-Bretagne et des infrastructures prévues pour le tunnel. Alors qu'il sera évincé par la compagnie mise en place pour mener le projet à partir du milieu des années 1870 , ce seront pourtant ses études qui serviront de référence pour les projets de la fin $\mathrm{du} \mathrm{xx}^{\mathrm{e}}$ siècle, notamment celui qui aboutit au tunnel actuel.

Fig. 1. - Projet Tomé de Gamond, 1867. F/14/12689.

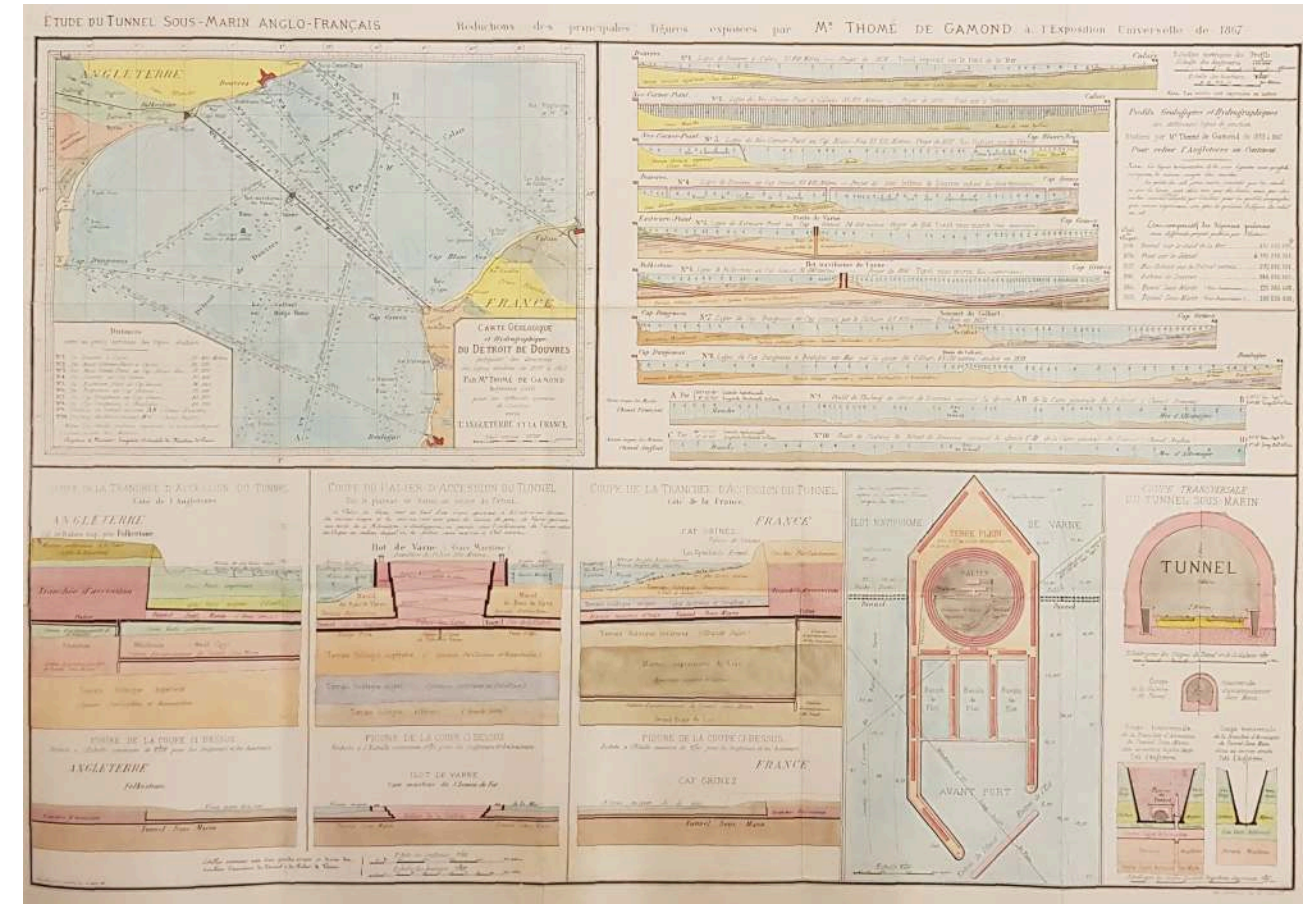

Arch. nat.

11 Présenté au nom de la compagnie fondée par MM. Low, Brumley, Hawkshaw et Thomé de Gamond, le projet est constitué d'un tunnel de 35400 mètres rattaché par des souterrains de dix kilomètres environ sur chaque rivage, au réseau des chemins de fer. John Clarke Hawkshaw découvre que la couche de craie bleue reste continue en s'enfonçant dans la mer. Elle pourra servir de veine pour le tunnel. William Low imagine deux tunnels à une voie, reliés par des rameaux transversaux pour la ventilation. Low, Brumley et Hawkshaw demandent le monopole de la concession de la ligne de chemin de fer passant par leur tunnel.

12 Les années qui suivent, de 1872 à 1888 , peuvent être considérées comme celle d'un vaste projet aux technologies de pointe aboutissant, malheureusement, à un rejet du côté britannique. L'entreprise "The Channel Tunnel Company », créée le 15 janvier 1872, est jumelée avec un comité formé en France au même moment: «La Société française du Tunnel sous la Manche ». Elles seront les ancêtres de la société qui relance le projet dans les années 1970, soit à l'issue de la concession de 99 ans qui leur est accordée par le ministère des Travaux publics en 1873. Suite à cette décision, une vaste enquête publique est organisée par les préfets.

13 L'enquête publique de 1873 est relayée dans toutes les communes du littoral du Pas-deCalais, comme l'attestent les avis rendus par les conseils municipaux transmis au 
ministère, ainsi que dans la presse ${ }^{8}$. Le projet de tunnel ayant des fondements purement économiques, l'enquête est aussi transmise à toutes les chambres de commerce de l'ensemble du territoire français. Elles adhèrent complètement au projet ou sous réserve d'un non monopole de la compagnie concessionnaire. Suite à cette enquête, plusieurs autres projets sont proposés au ministère des Travaux publics.

De juin à juillet 1874, le Conseil général des Ponts et Chaussées réunit une commission des communications entre la France et l'Angleterre. Elle est appelée à « exprimer son avis sur l'enquête ouverte dans le Pas-de-Calais, relativement à l'établissement du tunnel sous-marin projeté par M. Hawkshaw ${ }^{9}$. Cette commission a pour objectif d'étudier la faisabilité technique et financière du projet.

L'association du chemin de fer sous-marin entre la France et l'Angleterre est constituée

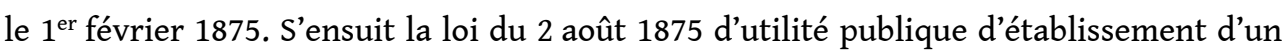
chemin de fer sous-marin entre la France et l'Angleterre et concédant l'exploitation de la ligne, côté français, à une compagnie. S'ensuit la constitution de la Commission internationale du chemin de fer sous-marin. Une convention est passée entre l'État et l'association. Une concession pour les études et les travaux préalables à la création du tunnel pendant cinq ans est accordée. Elle a pour objectif d'effectuer une reconnaissance hydrographique et géologique du terrain. Les relevés de Thomé de Gamond, décédé en 1876, semblent oubliés. Du moins, il n'en est pas fait mention dans les documents. En 1880, ces études ne sont pas achevées. Une demande de prorogation de trois ans est transmise au ministère. Plus de deux millions de francs sont alors engagés.

En 1878, à Sangatte, un puits de trois mètres de diamètre est creusé en vue de reconnaître le banc de craie. La société concessionnaire espère partir de ce puits pour creuser le tunnel. En 1880, 88,7 mètres ont été creusés. L'année suivante, un second puits est creusé, à partir du premier. Double de celui-ci, il atteint 5,4 mètres de diamètre. Tout un réseau de galeries souterraines destinées à reconnaître la couche de craie imperméable est constitué. Une galerie d'étude de près de 112 mètres de long part du premier puits (fig. 2). Côté britannique des puits et des galeries sont creusés entre Folkstone et Douvres. 
Fig. 2. - Photographie de la galerie creusée en 1888, 1975. 19770964/151.

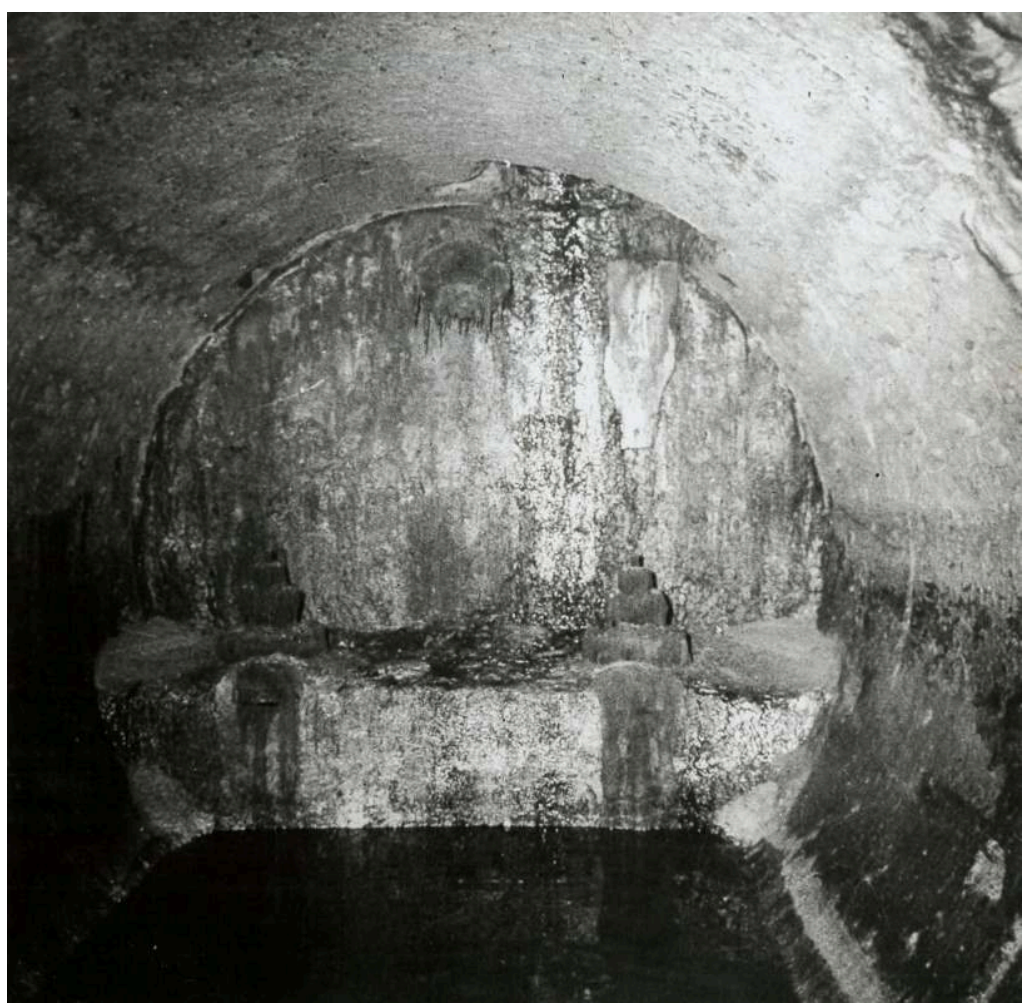

Arch. nat

17 Les archives conservées aux Archives nationales témoignent du contrôle financier exercé par l'État sur la Compagnie du chemin de fer sous-marin. Annuellement, la compagnie transmet son bilan financier via les Ponts et Chaussées du Pas-de-Calais. Le ministère des Travaux publics semble cependant conscient que l'avancée des travaux n'atteint pas le niveau escompté. Ainsi, on peut lire dans un des rapports fournis au ministre :

«On peut dire que toutes les dépenses faites appliquées réellement à des travaux d'études, ont été si sagement employées, que les travaux préliminaires exécutés auront leur utilité directe et pratique, si un jour le projet du chemin de fer sousmarin doit être réalisé ${ }^{10}$. »

Seulement, les différents projets sont bloqués en partie parce qu'il n'y a pas de concession similaire côté britannique. Mais ce sont surtout les réticences militaires britanniques qui bloquent finalement le projet. Le 28 juin 1888, la Chambre des Communes britannique rejette définitivement le projet, par crainte que le tunnel facilite l'invasion de l'île.

\section{Quand l'idée de franchir la Manche enflamme l'imagination (1873-1930)}

19 L'enquête publique lancée en 1873 provoque un élan de projets d'ingénieurs et de particuliers. En 1875, J. Castanier, ingénieur-constructeur, vice-président du conseil général du département du Rhône, transmet au ministère des Travaux publics son «nouveau projet de tunnel transmarin pour la traversée de la Manche et autres 
détroits ou bras de mer ${ }^{11} »$. Son projet reprend, à quelques détails près, celui de Thomé de Gamond, à la différence que l'île de Varne est prévue sur une île artificielle ronde, entourée d'un mur de protection. Là aussi, deux tunnels parallèles reliés par des tubes transversaux sont prévus. Cependant, le tunnel de Castanier n'est pas creusé dans le sous-sol. Il prévoit d'établir les tubes dans une enveloppe en béton monolithe reposant à même le sol de la Manche.

Des projets alternatifs au tunnel sont aussi proposés. En 1888, Lahaille présente un projet de téléphérique. Le schéma de ce projet présente un wagon utilisant les fils du télégraphe tendu entre deux tours pour se mouvoir à l'aide d'une roue sur deux chaînes tendues entre les deux tours.

21 La même année, François Martin, ingénieur, présente près d'une quinzaine de projets. Les calques d'une dizaine de ses propositions ont été conservés ${ }^{12}$. Tous ses projets proposent différentes façons de franchir la Manche: un "chemin de fer sous-marin coulant ", bac sous la forme d'un traîneau tiré par des chaînes et passant sur des roues fixées en haut de colonnes immergées; un "pont à patins voyageur " qui propose un bac accueillant un train et ses voitures, le tout mû par une chaîne embrayée par un système de roues dents fixées dans le bac, à ses extrémités et en haut de hautes tours immergées à intervalles réguliers (fig. 3). Son premier projet correspond cependant à plusieurs autres qui seront présentés plus tard. Il s'agit d'une voie ferrée avançant en mer jusqu'à des stations situées au large par lesquelles, à l'aide d'ascenseurs, les voyageurs embarquent dans un train souterrain, éclairés à l'électricité, les emmenant à un autre ascenseur leur permettant d'embarquer dans un train extérieur.

Fig. 3. - Projet de Pont à Patin, par François Martin, 1873, calque huilé. F/14/12689.

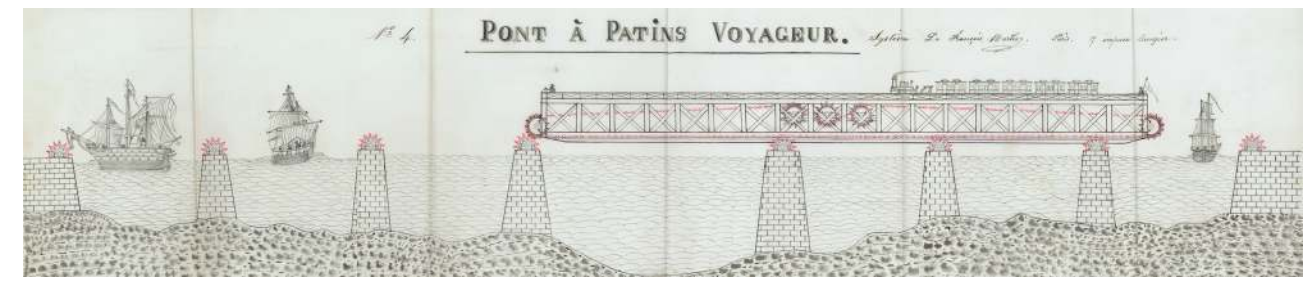

Arch. nat.

Suite aux réticences britanniques se développent des projets plus sécuritaires : des ponts, des systèmes avec des accès destructibles. En 1888, A. Boulat propose un système utilisant l'acide chlorhydrique pour détruire le train emprunté par les envahisseurs... ainsi que les passagers, tout en garantissant le nettoyage du tunnel après l'aspersion. Présenté deux ans plus tard, le projet Varilla est une variante du projet Martin de train menant jusqu'à des gares au large. Varilla propose de prolonger les voies françaises et britanniques en pleine mer sur des viaducs de deux kilomètres puis d'établir des « ouvrages convenablement protégés contre les flots » pour permettre la descente vers un tunnel «soit verticalement à l'aide d'ascenseurs, soit en pente à l'aide de plans inclinés ${ }^{13}$ ». Il garantit ainsi la possibilité de détruire les gares situées au large ainsi que les voies de chemin de fer d'accès, permettant ainsi de conserver l'infrastructure du tunnel.

En parallèle, pour garantir la sécurité des deux pays et éviter toute invasion, émergent des projets de ponts, plus facilement destructibles, ce qui a la faveur de la Grande- 
Bretagne, ou de procédés pour fermer le tunnel, comme le projet Barnett qui prévoit un procédé pour en dynamiter les issues.

La fin du XIX $x^{e}$ siècle voit aussi les projets Rothendt (1890), remorqueur mû par un câble sous-marin, Schubert (1894), tube sous-marin en fonte constitué de tronçons de 500 mètres réunis par des " membranes en tôle ou en caoutchouc ", ou encore le projet Della Santa (1896), tunnel ancré sous l'eau soutenu par quatre monticules artificiels surmontés de phares ${ }^{14}$.

25 Parmi les plus fervents adeptes à des projets de traversée de la Manche, Beau de Rochas figure en bonne place. Depuis les années 1860, il propose également des projets de tunnels. Parmi les fonds conservés aux Archives nationales, figure son projet de 1890 qu'il transmet au ministère des Travaux publics pour demander une concession provisoire pour la réalisation d'un «tunnel tubulaire à travers le détroit de la Manche ». Le ministère refuse en raison de la concession accordée à l'association du Tunnel sous la Manche.

26 Puisqu'il ne peut plus y avoir de concession accordée pour un tunnel, ni pour une ligne de chemin de fer, plusieurs projets proposent des projets alternatifs. Outre les projets Lahaille et Martin présentés plus haut, des projets de chaussées traversant l'intégralité de la Manche ou de ponts voient le jour.

Ainsi, M. Passedoit présente le projet de création entre le cap Gris-Nez, dans le Pas-deCalais, et Folkestone d'un "barrage maçonné livrant passage aux navires par deux passes doubles, munies de ponts tournants ${ }^{15}$ " à proximité des côtes française et britannique. Vingt ouvertures de 50 mètres de large permettent le passage en haute mer. Sur ce barrage, M. Passedoit prévoit une chaussée de 25 mètres de large, à 6 mètres au-dessus des plus hautes mers, sur laquelle une voie carrossable et trois voies de chemin de fer. Comme il l'explique dans son mémoire :

«Ce qui pourrait, parfois, permettre aux embruns de s'y faire un peu sentir aux voyageurs ${ }^{16}$.»

28 Le projet n'est pas retenu. Outre la question de la concession, le temps nécessaire à la construction d'un tel ouvrage en rend la réalisation inenvisageable. Il est aussi intéressant de noter que, parmi les arguments avancés par le ministère des Travaux publics pour refuser ce projet, des préoccupations de sauvegarde de l'environnement, notamment de préservation des courants de la Manche qui seraient contrariés par la chaussée, et donc de l'écosystème, sont aussi présentées.

Dès 1870, les projets de ponts vont se multiplier. De 1870 à 1883, J.-D. Vérard de SainteAnne, ingénieur, propose plusieurs projets qui évoluent pour aboutir à celui d'un "chemin de fer à ciel ouvert, entre l'Angleterre et la France", constitué de quatre viaducs métalliques menant à une île centrale sur laquelle figure en bonne place une gare majestueuse desservie par une ligne de 36 kilomètres permettant une traversée en " 35 à 40 minutes $^{17}$ ". Son projet ne sera pas, non plus, accepté.

En 1889, la Channel Bridge and Railway Company propose un projet, qu'elle revoit après un premier refus en 1891, d'un pont métallique d'une longueur de $33,45 \mathrm{~km}$, soutenu par 72 piles séparées par des travées de 300 à 400 mètres (fig. 4). Projet très abouti, il est étayé par plusieurs pré-projets, notamment l'avant-projet Hersant pour les piles, Schneider et Cie pour la superstructure métallique et Sautter et Harlé pour la signalisation maritime. Le projet obtient le soutien de la majorité des conseils généraux départementaux, y compris le conseil général du département d'Oran, qui y voient un 
moyen simple, moderne et sans risque de transport des marchandises de Suez à Londres. Là aussi, la durée du chantier, de près de quinze ans pour 380 millions de francs, provoque le refus du Conseil général des Ponts et Chaussées. Face au refus, la compagnie, dépitée, propose un bac, plus simple et moins coûteux, qui sera aussi refusé.

Fig. 4. - Projet de la Channel Bridge and Railway Company, 1893. F/14/12692.

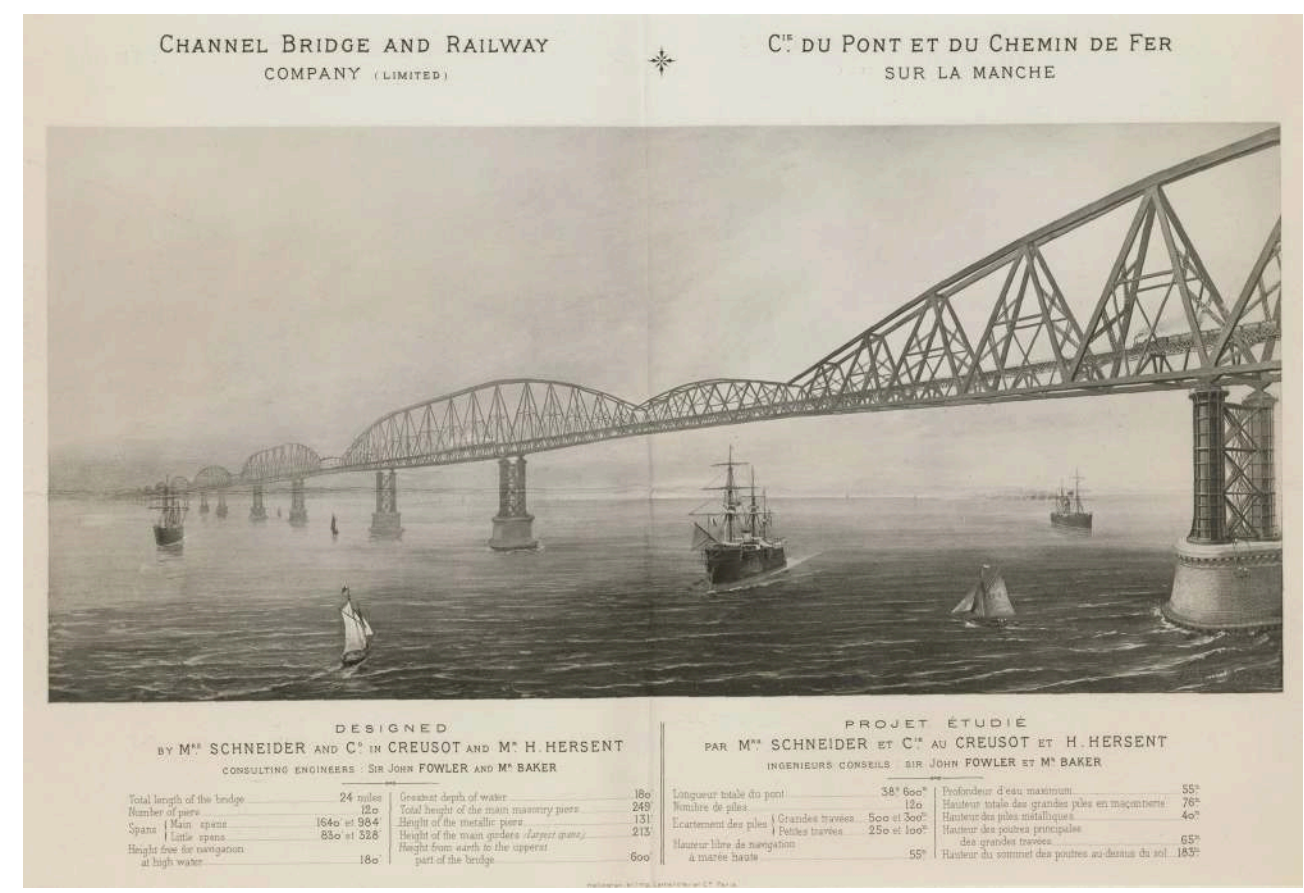

Arch. nat.

La Première Guerre mondiale et l'Entente cordiale ravivent le projet d'un tunnel sous la Manche des deux côtés de la mer. De nombreux élus, les Chambres de commerce et plusieurs municipalités informent le gouvernement français que le projet serait profitable à la Nation. La presse, des deux côtés de la Manche, voire outre-Atlantique, se fait l'écho du projet. Le tunnel sous la Manche devient un outil du rapprochement entre la France et la Grande-Bretagne. Le Conseil supérieur des Travaux publics, après avoir pris connaissance du rapport de M. Sartiaux, constitue un comité du Tunnel sous la Manche. L'étude du projet est reprise en 1921, sans suite.

Pendant l'entre-deux-guerres, plusieurs projets sont proposés, favorisant plutôt le pont. On peut citer le projet de Jules Jaeger (fig. 5), ingénieur à Fribourg, en 1930, constitué d'une double jetée formant chenal et viaducs pour routes et voies ferrées. Il s'agit de $42 \mathrm{~km}$ de voies séparées l'une de l'autre de $300 \mathrm{~m}$. Y sont prévues des doubles voies ferrées auxquelles serait superposée une chaussée pour automobiles et camions. La séparation entre les deux jetées formerait alors un canal pour une navigation tranquille et offrant un abri en cas d'attaques sous-marines. On peut y voir là l'influence des destructions de la Première Guerre mondiale. 
Fig. 5. - Projet de Jules Jaeger, 1930. 19870693/2.

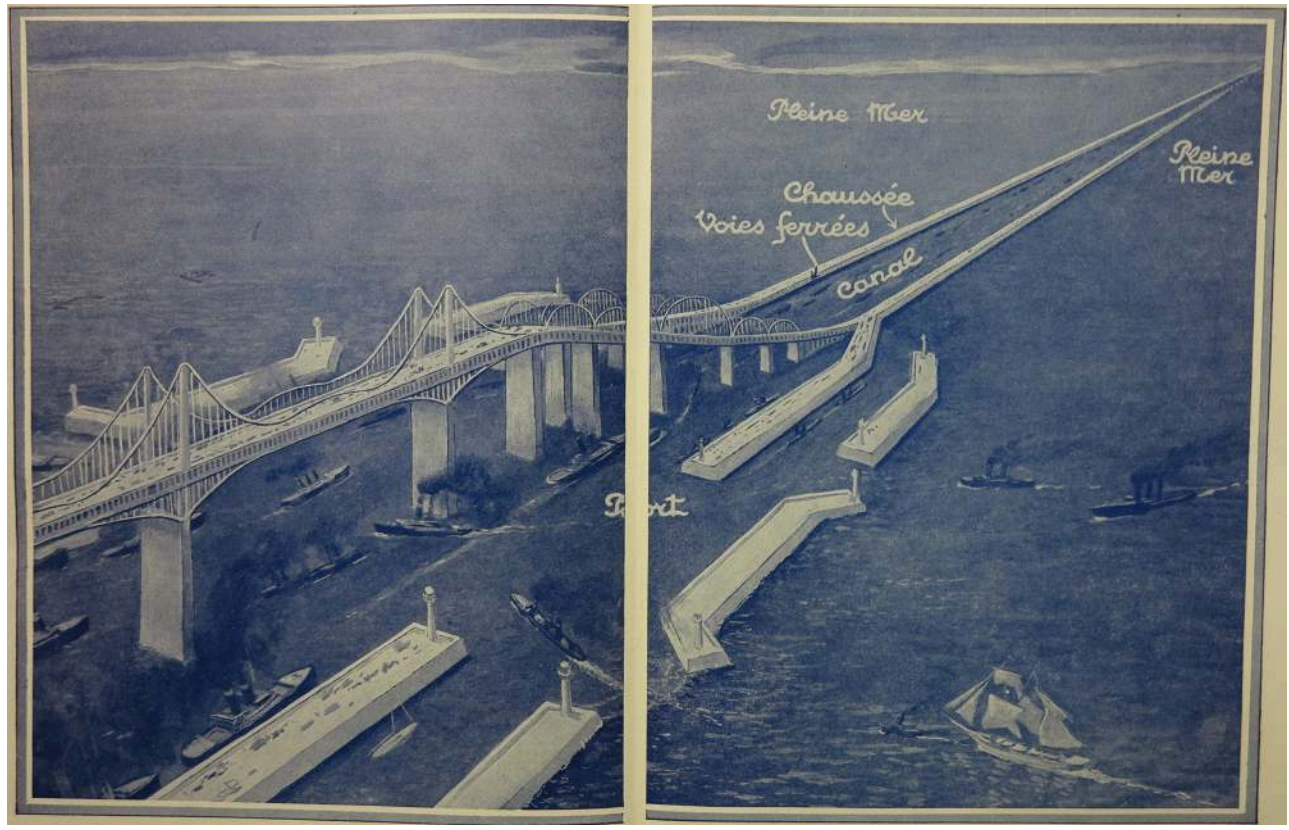

Arch. nat.

Deux autres projets sont proposés dans les années 1930 : un projet de pont-tunnel par M. Mahl et un projet de pont par M. Crush. Tous ces projets successifs montrent l'intérêt croissant, au niveau international, d'un ouvrage, quel que soit, permettant de franchir la Manche. Les événements de la moitié du $\mathrm{xx}^{\mathrm{e}}$ siècle coupent court à tout projet. Il faut attendre la fin des années 1950 pour que soit relancée l'idée d'un tunnel sous la Manche.

\section{Le virtuel se concrétise : les tunnels de la seconde moitié du $x x^{e}$ siècle}

Ce sont, en fait, les sociétés qui ont obtenu la concession en 1875 qui relancent le projet. À la suite de l'abandon du projet en 1888, l'étude est reprise en 1904 puis en 1921 sans qu'il y ait cependant un début de réalisation. C'est en 1957 que le projet est relancé avec la constitution du Groupement d'Études du Tunnel sous la Manche (G.E.T.M.), sous l'égide de la compagnie financière de Suez, de la Channel Tunnel investments Ltd, de la Société concessionnaire et d'une société américaine "Technical studies inc.». Un premier programme d'études géologiques et d'études de trafic est réalisé. Durant les années 1960, le G.E.T.M. présente un projet de tunnel, que les gouvernements français et britannique acceptent en 1964. Les années suivantes sont consacrées aux études géologiques, aux campagnes de forage en mer et à l'élaboration du coût et du planning du projet.

En 1967, un appel d'offres est lancé par les deux gouvernements, français et britannique. Le G.E.T.M. est désigné maître d'œuvre le 22 mars 1971. Les travaux commencent en 1973 et un traité franco-britannique est signé et ratifié en décembre 1974 par le parlement français. À ce titre, la Délégation française au tunnel sous la Manche est créée le 19 mai 1974. Cette délégation abroge le groupe de travail 
interministériel pour l'étude de la réalisation du tunnel sous la Manche (1966-1974) et la Société du tunnel sous la Manche (1971-1975) qui sont tous deux regroupés au sein de la nouvelle entité.

Le projet est présenté comme le digne hériter des projets précédents, notamment ceux de Thomé de Gamond et de Law auxquels il est fait référence dans les dossiers de présentation. Comme le montrent les photographies de la présentation du projet ${ }^{18}$, les références aux projets précédents sont primordiales. Le projet des années 1970 est profondément ancré dans les précédentes études, notamment pour démontrer le savoir-faire du G.E.T.M.

Il est cependant résolument tourné vers l'avenir en prenant notamment en compte l'environnement immédiat du futur terminal qui est intégré dans le paysage tout en lui attribuant une empreinte volontairement futuriste et imposante. Il est également le premier projet à intégrer la conception d'un transport spécifique au tunnel sous la Manche prévoyant deux types de wagons: pour véhicules et voyageurs et pour véhicules seulement.

38 Cependant, le Royaume-Uni est plongé dans une crise économique et abandonne une fois de plus le projet le 20 janvier 1975. Mais les projets de pont persistent, notamment avec celui de la Société d'Étude du Pont sur la Manche de 1961.

39 En mai 1981, le projet est relancé. Quatre dossiers sont présentés: Eurobridge ou Europont, le TransManche Express, Euro-Route et Eurotunnel ${ }^{19}$. Tous volontairement implantés dans la modernité, ils présentent, dans une trame assez commune, des projets qui rivalisent, parfois à l'extrême, dans le futurisme.

40 Le projet Europont présente un pont-tube de $37 \mathrm{~km}$ soutenu par huit pylônes de $340 \mathrm{~m}$ de hauteur, faisant appel à des techniques nouvelles, avec des travées longues de $5 \mathrm{~km}$ suspendues à des câbles en kevlar. Le pont aurait deux niveaux de six voies chacun. Une liaison ferroviaire serait faite par un tunnel. Le coût était évalué à 68 milliards de francs.

41 Le projet d'Euroroute est constitué d'un ensemble routier pont-tunnel-pont. Les ponts à haubans avec des travées de $500 \mathrm{~m}$ de portée relient des îles artificielles à la côte, et un tunnel de $21 \mathrm{~km}$ sous le fond de la mer. Des rampes hélicoïdales permettent le passage du pont au tunnel. Une liaison ferroviaire indépendante passe par deux tunnels. Le coût était évalué à 54 milliards de francs hors frais financiers.

42 Celui de Transmanche Express a été présenté à la dernière minute par la société British Ferries. Il comprenait un ensemble de quatre tunnels (deux routiers et deux ferroviaires) unidirectionnels. Deux îles artificielles seraient créées pour assurer la ventilation des tunnels routiers via des puits. Le coût annoncé est de 30 milliards de francs.

Enfin, puisqu'il s'agit du tunnel actuel, le projet Eurotunnel est, dans ses grandes lignes, celui de 1972-1975, à savoir un double tunnel ferroviaire avec un troisième tunnel de service. Ce projet a un coût estimé à 30 milliards de francs.

Le projet Eurotunnel est retenu. En parallèle, la Délégation au tunnel sous la Manche devient le Secrétariat général au tunnel sous la Manche. Le traité de Cantorbéry est signé le 8 septembre 1987 et retranscrit par le décret n 87-757 du 8 septembre 1987, portant publication du traité entre la République française et le Royaume-Uni de Grande-Bretagne et d'Irlande du Nord concernant la construction et l'exploitation par des sociétés privées concessionnaires d'une liaison fixe transmanche. 
Les travaux commencent en 1987 et s'achèvent en 1993. Tous les mois, le ministère des Travaux publics reçoit un journal d'avancée du chantier. Ces petits livrets, conservés aux Archives nationales dans le versement 20080257, présentent les schémas techniques des différents tronçons réalisés, les difficultés rencontrées et, surtout, sont illustrés de photographies des différentes étapes. La jonction entre les deux tunnels s'effectue le $1^{\mathrm{er}}$ décembre 1990. Le tunnel sous la Manche est officiellement inauguré par la reine Élisabeth II et le président François Mitterrand le 6 mai 1994.

Les archives relatives au tunnel sous la Manche versées aux Archives nationales depuis le milieu des années 2000 témoignent de l'avancée de ce projet et proposent aux chercheurs des sources pour l'histoire des techniques. Elles témoignent aussi de l'implication de l'État français dans la gestion de l'établissement, ainsi que, indirectement, des relations franco-britanniques depuis le XIX ${ }^{\mathrm{e}}$ siècle.

Il aura donc fallu plus de deux siècles pour voir se concrétiser un rêve resté longtemps du monde du virtuel. Des premiers projets, qui pourraient être tirés d'un des meilleurs ouvrages de Jules Verne, dont ils sont contemporains, en passant par les initiatives privées poussant certains, comme Thomé de Gamond, à l'extrême, jusqu'à la rencontre des ouvriers français et britanniques en 1990, ce sont les efforts de milliers d'hommes et de femmes qui se sont concrétisés dans ce vaste projet. Les archives du tunnel sous la Manche conservées aux Archives nationales permettent ainsi de se plonger dans le rêve de ces hommes de la fin du XIX ${ }^{e}$ siècle et de tout le $\mathrm{XX}^{\mathrm{e}}$ siècle, dans ces projets successifs, parfois fantasques, restés au niveau du virtuel, pour ressurgir dans le présent et la réalité du tunnel sous la Manche tel que nous le connaissons aujourd'hui.

\section{NOTES}

1. Arrêté du 24 décembre 2006 érigeant le service Archives nationales en service à compétence nationale, article 2.

2. Il s'agit des articles F/14/8593 relatif au chemin de fer sous-marin entre la France et l'Angleterre (1875), F/14/12689 à F/14/12691 concernant les projets divers du tunnel sous la Manche et la Compagnie du tunnel sous-marin (1867-1919), ainsi que les articles F/14/12692 et F/14/12693 présentant les projets de ponts sur la Manche (1870-1896).

3. Par ordre chronologique, il s'agit des versements et articles suivants : 19770964, 19770984, associé au 19860475, 19771001, article 19771012/13, article 19780603/7, article 19810023/6, article 19850731/4, 19870693, article 19890265/14, 19890272, 20080257, 20160142.

4. Notamment les articles 19850731/4, 19890265/14. 
5. Pour plus d'informations sur la SFTM, consulter l'introduction de l'inventaire du versement 19770964: https://www.siv.archives-nationales.culture.gouv.fr/siv/IR/ FRAN_IR_017193

6. Pour plus d'informations sur la Commission intergouvernementale du tunnel sous la Manche, consulter le site de la CIG: http://www.cigtunnelmanche.fr . Consulter également l'introduction de l'inventaire du versement 201602142: https:// www.siv.archives-nationales.culture.gouv.fr/siv/IR/FRAN_IR_054975

7. Décret $n^{\circ} 87-757$ du 8 septembre 1987 portant publication du traité entre la République française et le Royaume-Uni de Grande-Bretagne et d'Irlande du Nord concernant la construction et l'exploitation par des sociétés privées concessionnaires d'une liaison fixe trans-Manche. NOR : MAEJ8730W2D (Journal officiel du 16 septembre 1987, page 10769).

8. $F / 14 / 12691$.

9. $\mathrm{F} / 14 / 12691$.

10. $\mathrm{F} / 14 / 12890$.

11. $\mathrm{F} / 14 / 12689$.

12. $\mathrm{F} / 14 / 12689$.

13. $\mathrm{F} / 14 / 12689$.

14. $F / 14 / 12689$.

15. $\mathrm{F} / 14 / 12692$.

16. $\mathrm{F} / 14 / 12692$.

17. $\mathrm{F} / 14 / 12692$.

18. Conservées dans le versement 19770964.

19. Consulter le versement 20080257 pour découvrir l'ensemble de ces projets.

\section{RÉSUMÉS}

La Manche a longtemps représenté une barrière naturelle entre le continent et les îles britanniques. Objet de convoitise dès l'Antiquité, elle s'inscrit dans l'imaginaire collectif comme une "frontière naturelle». Dès le XVIII ${ }^{e}$ siècle, des ingénieurs imaginent des moyens techniques pour en permettre un franchissement non maritime. Pont, liaison aérienne, îles artificielles font place à plusieurs projets de tunnels qui avortent face aux conflits successifs qui touchent l'Europe durant les $\mathrm{XIX}^{\mathrm{e}}$ et $\mathrm{Xx}^{\mathrm{e}}$ siècles. Symbole de la création de la Communauté économique européenne, le projet de tunnel sous la Manche se met en place au début des années 1970 et est inauguré en 1994. Les archives relatives à ce vaste projet sont conservées aux Archives nationales. Après une présentation de l'origine et de la nature de ces archives sont abordés les projets successifs qui ont permis la concrétisation d'un rêve de plusieurs siècles devenu réalité. 
AUTEUR

STÉPHANE RODRIGUEZ-SPOLTI

Responsable de fonds du ministère des Travaux publics et des Transports, Archives nationales 


\title{
Relier les collines de Fourvière et de la Croix-Rousse à Lyon (1847-1990)
}

\author{
Françoise Bayard
}

1 Fourvière et la Croix-Rousse sont deux collines de granit et de gneiss de 250 et 295 mètres d'altitude séparées par la Saône (fig. 1). La première a été le berceau de la cité : les Romains y avaient installé leur camp puis leur ville. Au Moyen Âge, on y a construit une chapelle qui a reçu en 1852, une statue de la Vierge sculptée par Fabisch à l'origine des illuminations de la ville. En 1810, une nécropole y a été créée. La colline de Fourvière permet l'accès aux Monts du Lyonnais ruraux et au-delà aux mines de houille de la région stéphanoise utiles aux industries sidérurgiques et à la liaison ferroviaire Lyon Saint-Étienne mise en place entre 1826 et 1836. La Croix-Rousse a été une commune indépendante jusqu'en 1852, date à laquelle elle a été incorporée à la capitale des Gaules. S'y sont installés les Canuts travaillant la soie sur les métiers Jacquard. Ils ont déserté le centre-ville, secteur bouleversé par les travaux d'hausmannisation du préfet-maire Vaïsse jusqu'à sa mort en 1864. Ils se sont révoltés en 1831 pour des raisons économiques et en 1834 pour des raisons politiques, mouvement républicain qui s'est à nouveau manifesté lors de la Révolution de 1848 et de la Commune en 1870. 
Fig. 1. - L'emplacement des ponts (figuré en noir), entre les collines de Fourvière (à gauche de la Saône) et de la Croix-Rousse (à droite de la Saône).

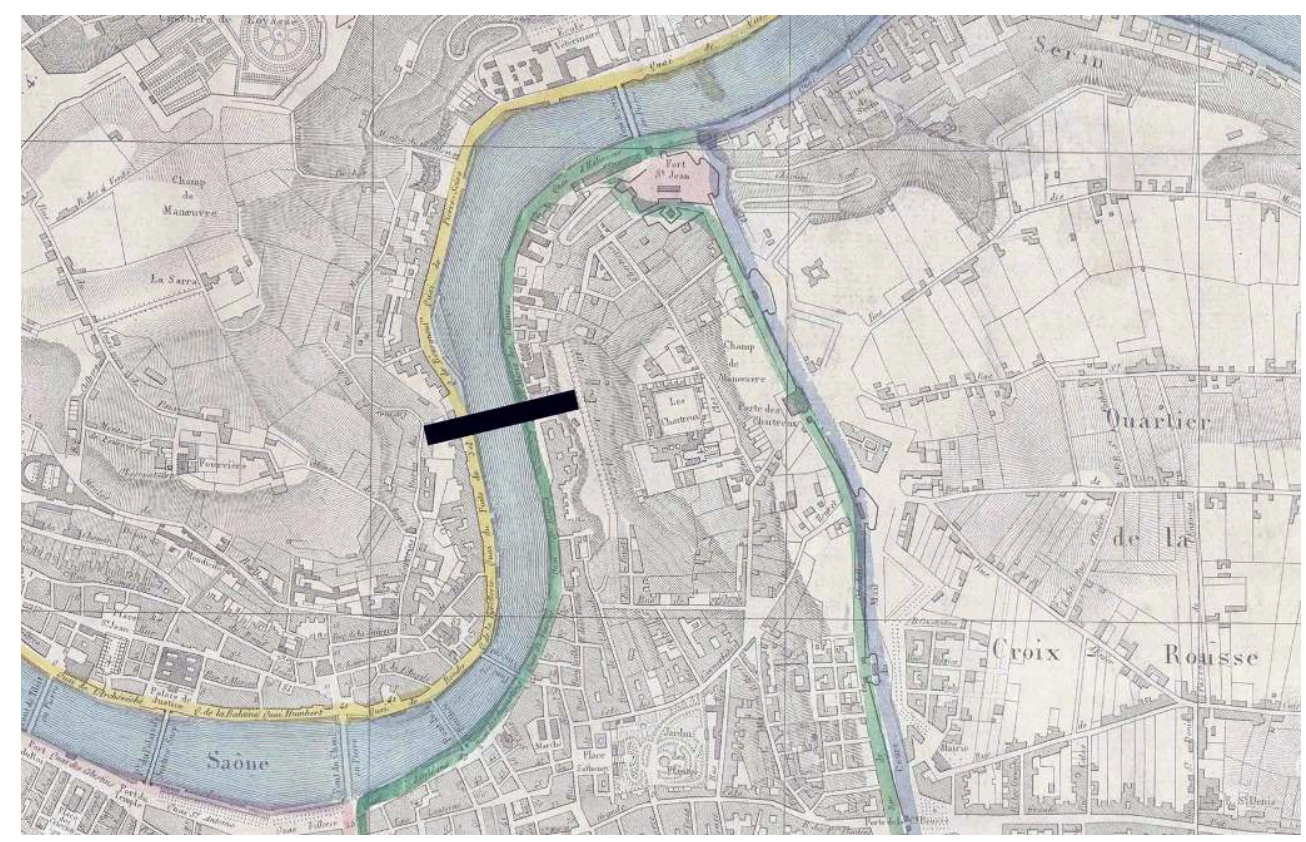

Extrait de arch. mun. Lyon, 2S 264, «Plan de Lyon et de ses nouveaux quartiers... », 1858.

2 L'idée de relier la Croix-Rousse au centre ne pouvait que germer chez les Lyonnais : ne serait-ce que pour contrôler le travail des Canuts, les maisons de soierie étant restées au bas des pentes. D'où l'idée d'un pont. La Saône dispose ici d'une vallée de 100 à 120 mètres de large, sa pente est faible, son courant peu rapide, son débit abondant et son régime contrasté : elle s'évapore l'été et " gonfle » l'hiver. L'emplacement choisi n'a rien d'original. En 1789, alors que le pont de Serin vient de s'écrouler, l'architecte Morand propose de le reconstruire à l'emplacement de l'Homme de la Roche (fig. 1) en raison des roches apparentes et de l'étroitesse du défilé ( 90 mètres) ${ }^{1}$ créant ici « les rapides de la mort qui trompe " alors qu'ailleurs la profondeur du lit est inférieure à dix mètres et le fond constitué de sables fins et d'argile². Après la Révolution et jusqu'en 1990, selon un rythme très inégal, il a été suivi par 34 individus ou entreprises qui ont présenté 44 projets divers, toujours localisés au même endroit. Si leurs objectifs sont différents, le cheminement de leurs démarches est assez semblable. Au bout du compte, après une période d'enthousiasme, pour des raisons variées, leurs projets ne sont pas réalisés et restent donc à l'état de documents virtuels qu'on peut retrouver pour le $\mathrm{XIX}^{\mathrm{e}}$ siècle dans les archives municipales lyonnaises et pour le $\mathrm{XX}^{\mathrm{e}}$ siècle dans différents dépôts ${ }^{3}$.

\section{Les auteurs des projets}

Ils sont très différents les uns des autres (tabl. 1).

Tabl. 1. - Liste chronologique des auteurs et de leurs projets.

1847 - Lehaître et Bonnardet

1847 - Chipier 1 


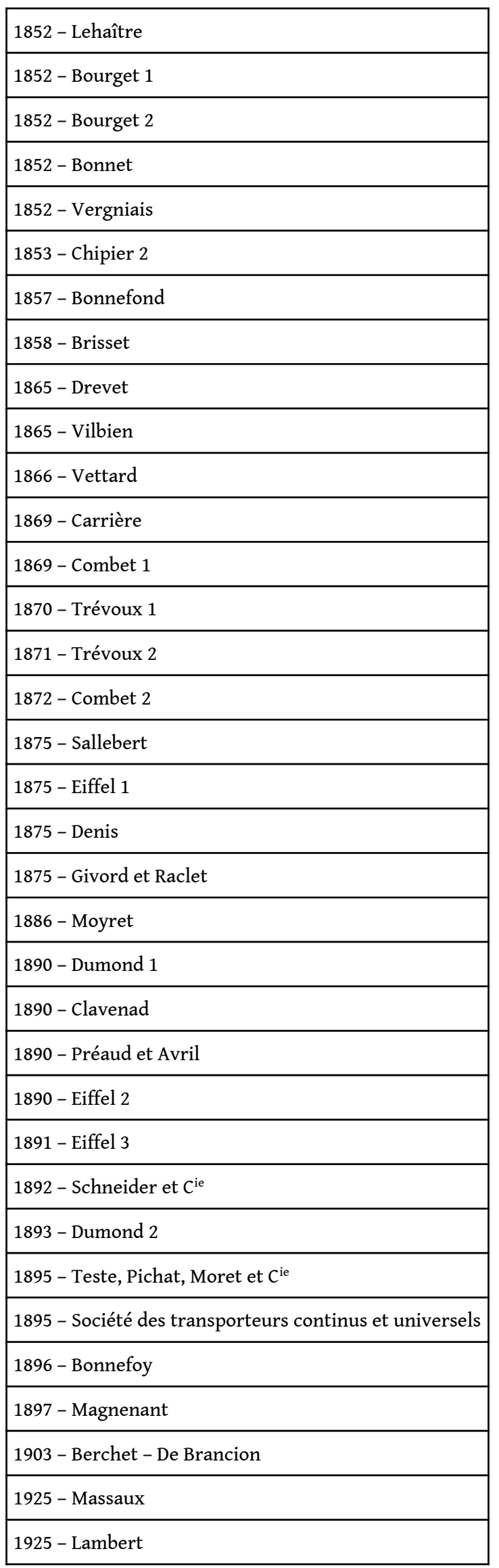




\begin{tabular}{|l|}
\hline 1946 - Lambert \\
\hline 1986 - Jourda, Perraudin, Rice \\
\hline 1987 - Faucon \\
\hline 1990 - Mimram \\
\hline 1990 - Jourda-Perraudin \\
\hline 1990 - Berlottier \\
\hline 1990 - Foster \\
\hline
\end{tabular}

Doc. F. Bayard.

4 Vingt et un auteurs sont des personnes seules, comme Bonnet ou Sallebert ${ }^{4}$, quand treize sont des industriels : ainsi Gustave Eiffel et compagnie, Schneider et compagnie et Teste fils, Pichat, Moret et compagnie des Tréfileries et Câbleries de Lyon Vaise ${ }^{5}$. On connaît les professions de trente-cinq d'entre eux. Quatorze se disent ingénieurs dont quatre ingénieurs civils, cinq ingénieurs des ponts et chaussées et un ingénieur chimiste $^{6}$. Douze sont architectes à l'instar de Chipier, Magnenant ${ }^{7}$ et les constructeurs $\mathrm{du} \mathrm{xx}^{\mathrm{e}}$ siècle (Faucon, Jourda, Perraudin, Rice, Berlottier, Mimram, Foster). Six sont métallurgistes, ainsi Bonnefoy gérant de la société technique d'études du câble transbordeur8. Trois autres exercent des professions diverses (maître tailleur; entrepreneur en charpente ; médecin) ${ }^{9}$.

5 Sur les trente dont on connaît le lieu de résidence, quatorze habitent Lyon comme Brisset $^{10}$; cinq sont domiciliés à Paris ${ }^{11}$. Onze autres résident dans la banlieue lyonnaise à l'instar de Préaud ${ }^{12}$ ou différentes régions de France ainsi Corneillan en Gironde pour Denis $^{13}$ et au $\mathrm{xx}^{\mathrm{e}}$ siècle, la Grande-Bretagne pour Rice et Foster. Ils mentionnent alors leur hôtel à Lyon, ou leur adresse de vacances.

6 Pour se mettre en valeur, certains rappellent leurs attaches avec la ville. D'autres mentionnent leurs titres ou leurs travaux. Aucun cependant n'a le curriculum vitae de Clavenad : à l'École des ponts et chaussées où il a étudié de 1873 à 1876, il a obtenu les prix de mécanique, des ponts et d'architecture. Il a travaillé ensuite au service des travaux hydrauliques de la marine. En 1879-1880, il a participé à la mission gouvernementale pour le chemin de fer transsaharien en Algérie. Chef des études techniques de la compagnie du canal interocéanique de Panama, il en a pris la direction des études en 1880-1881. En 1882, au service central technique du ministère de l'Agriculture à Paris, on lui confie une mission générale en Algérie après la rupture d'un barrage. En 1883, il retourne au Panama comme ingénieur en chef des travaux et élabore le projet définitif du canal et de ses dérivations, lance le pont de la ville nouvelle de Colon ainsi que les ateliers, les magasins généraux et l'agence maritime. En tant que directeur des services lyonnais, il a fait quatre ponts, une nouvelle distribution de l'eau et amélioré la rue Grolée. Ses travaux scientifiques portent sur le Sud oranais, la propagation de la marée, la stabilité des massifs en général, des murs de soutènement et des barrages, la théorie mécanique de la réflexion et de la réfraction de la lumière, la filtration ${ }^{14}$. Teste fils, Pichat, Moret et Compagnie rappellent les médailles obtenues par leur entreprise (1872, médaille d'or de l'exposition universelle; 1878, médaille d'or à Paris ; 1887, diplôme d'honneur au Havre ; 1889, deux médailles d'or à 
Paris ; 1894, deux grands prix à Lyon $\left.{ }^{15}\right)$. Enfin, certains soulignent leur attachement au régime en cours.

7 Vingt-six comme Berchet ${ }^{16}$ ne font qu'une proposition, mais Jourda et Perraudin en font deux et Eiffel, trois, ce qui prouve leur volonté de les voir aboutir. Certains, ainsi Combet qui explique qu'il «a abandonné le premier projet » ou Eiffel affirmant qu'il est «tout disposé à faire des propositions sur ce sujet » font des modifications au dessein initial. La simultanéité de certains projets ne peut que poser la question de leur spontanéité. Les années 1840 en voient deux; la décennie suivante, huit; les années 1860, cinq ; les seules années 1875 et 1886, quatre et un ; la décennie 1890, onze ; 1903, un ; les années 1920, deux ; 1946, un ; les années 1980, deux et l'année 1990, quatre (fig. 2).

Fig. 2. - Répartition annuelle des projets.

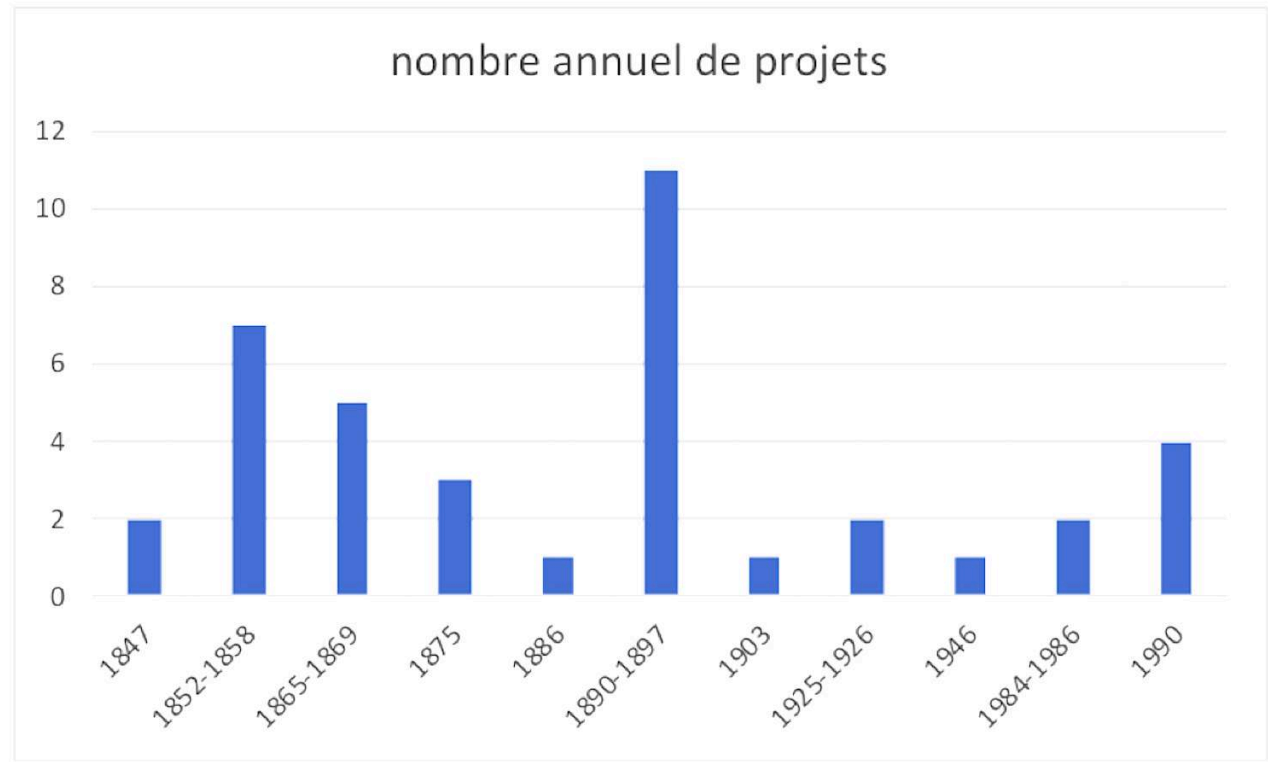

Doc. F. Bayard

Sauf en 1990, année où la Communauté urbaine de Lyon lance un concours, très peu d'indications laissent penser qu'il y a eu compétition organisée à un moment donné. Tout au plus, Préaud et Avril déclarent-ils que "la question est à l'ordre du jour ${ }^{17}$ ». Dans la Lettre du génie civil, revue générale hebdomadaire des industries françaises et étrangères, Maxime de Nansouty suggère que la société Eiffel a travaillé en 1891 «à la demande de la municipalité » et « qu'il a fait un contre-projet » à celui de Clavenad ${ }^{18} \mathrm{ce}$ que ne contredit pas ce dernier :

«J'ai été chargé par la municipalité de dresser un projet de viaduc lequel a été adopté par elle et visé19.»

Le Journal franco-californien va même jusqu'à écrire :

«Le conseil municipal avait mis au concours un projet pour la construction d'un grand viaduc ${ }^{20}$."

Quoi qu'il en soit, la presse vante, selon ses opinions, trois projets apparus au même moment en 1890. On remarque néanmoins que les auteurs se sont particulièrement investis au moment de l'union des deux communes et ont été moins prolifiques après la crise de 1880. Sauf Dumont qui, en 1893, ajoute un immense viaduc dit de Saint-Laurent 
reliant les deux plateaux aux Brotteaux ${ }^{21}$, tous ces projets visent la construction d'un pont sur le même emplacement. Mais ils sont différents les uns des autres.

\section{Quel pont pour quel Lyon ?}

11 Décrivant leurs procédés, les concepteurs vantent souvent leur nouveauté : Vergnais a conçu « le pont d'Hercule » (fig. 3) ; Eiffel parle du « système Cantilever » ou « console » (fig. 4) 22. D'autres citent le modèle dont ils s'inspirent, ainsi Hector Vilbien: "pont rigide à armature à réaction automatique inventé par l'abbé Castay et employé pour le pont du château de Mazères ${ }^{23}$ » ou ceux qui ont déjà été réalisés : le pont de La RocheBernard sur la Vilaine et de Fribourg pour Lehaître ; Britannia par Stephenson, Conway, Dirschau sur la Vistule pour Givord et Raclet. Ces précautions prises, les œuvres projetées se diversifient par leurs dimensions, leurs hauteurs sur la rivière et les matériaux utilisés : seul Carrière propose la construction d'un pont en bois (fig. 5) ; Vergnais et Vilbien utilisent le fer et la fonte; Drevet et Combet la pierre et au $\mathrm{xx}^{\mathrm{e}}$ siècle Massaux et Trévoux, le ciment armé. Seize autres souhaitent employer le métal, huit sous la forme de ponts suspendus (fig. 6), dix sous celle de pont métallique ${ }^{24}$ utilisant différents métaux, l'acier, dont la production se perfectionne à cette époque. Ils sont constitués par l'assemblage de poutres à âmes pleines ou à treillis (fig. 7). Au $\mathrm{xx}^{\mathrm{e}}$ siècle, quatre autres ponts sont à haubans (fig. 8) ${ }^{25}$. Leurs formes sont différentes. La plupart n'ont qu'une arche, mais celui de Combet en a deux et ceux de Givord et Raclet et de Lambert, trois. Les plus nombreux ont un seul étage, mais six en possèdent deux permettant une double circulation (dont une ferroviaire pour Clavenad) au niveau des rives de la Saône et à celui des collines (fig. 9). Faucon a prévu un pont habité. Pratiquement tous joignent un ou plusieurs dessins ou plans à leur demande.

Fig. 3. - Le pont d'Hercule.

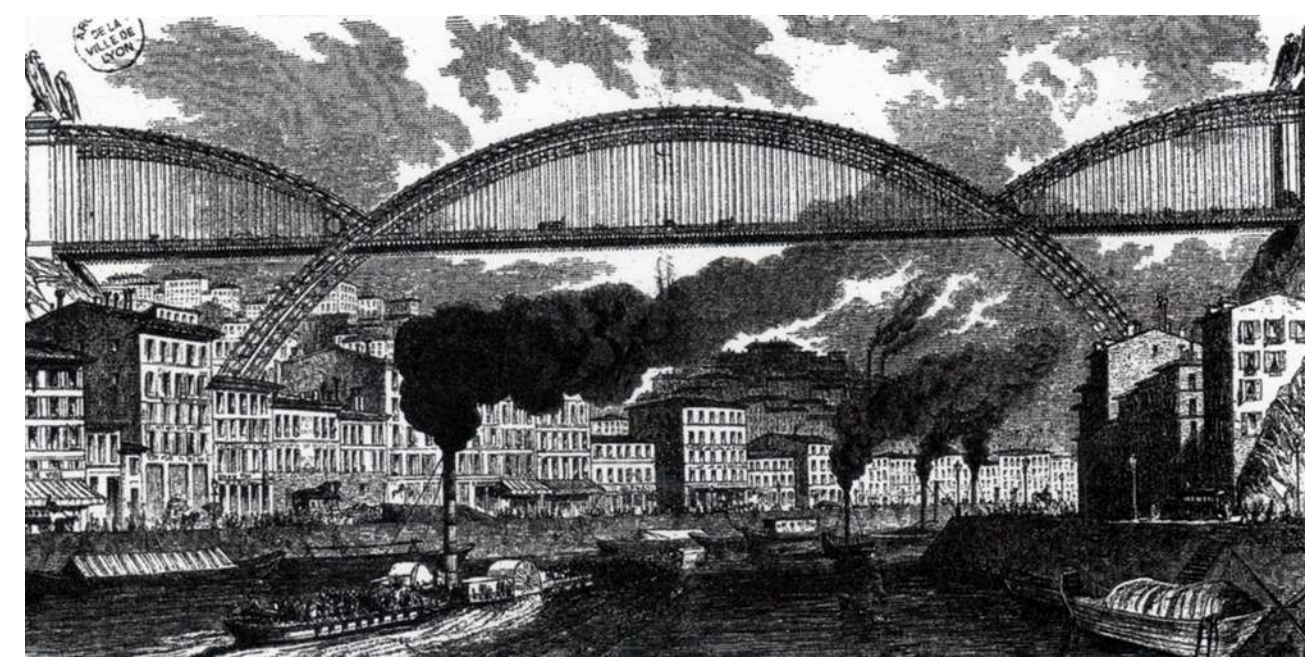

Doc. V. Giorgiutti. 
Fig. 4. - Le procédé Cantilever : l'exemple du pont de Québec (vu de l'est).

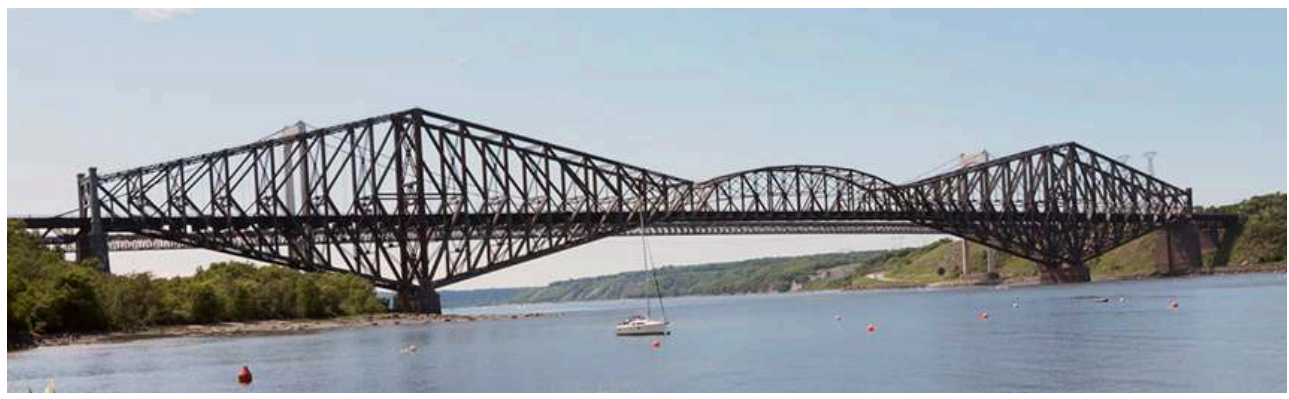

Cliché Muriel Leclerc @ .

Fig. 5. - Le pont de bois.

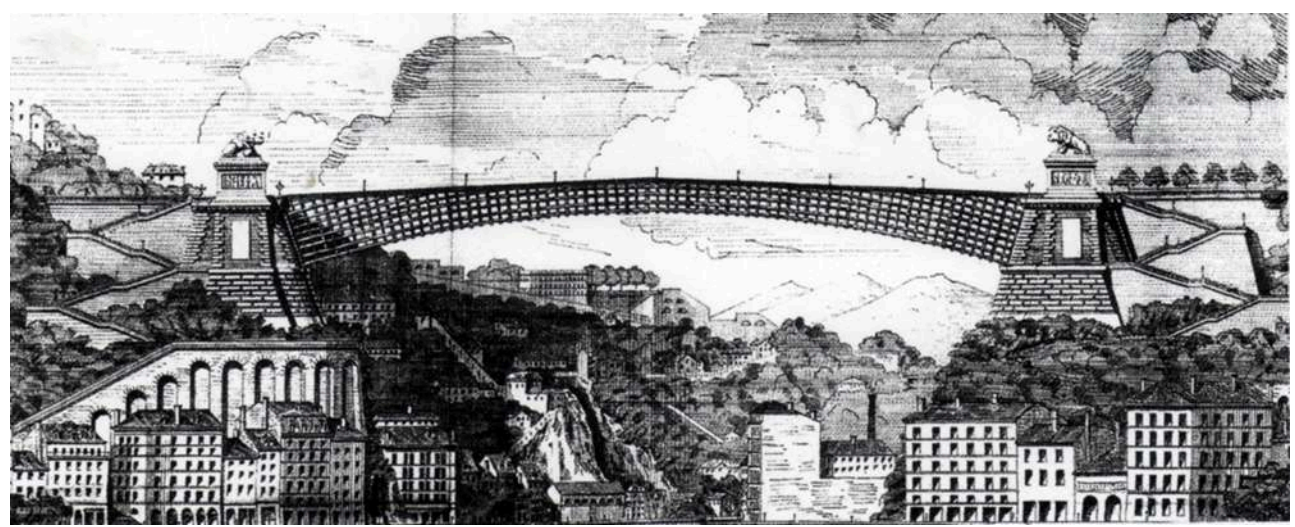

Doc. V. Giorgiutti.

Fig. 6. - Un pont suspendu.

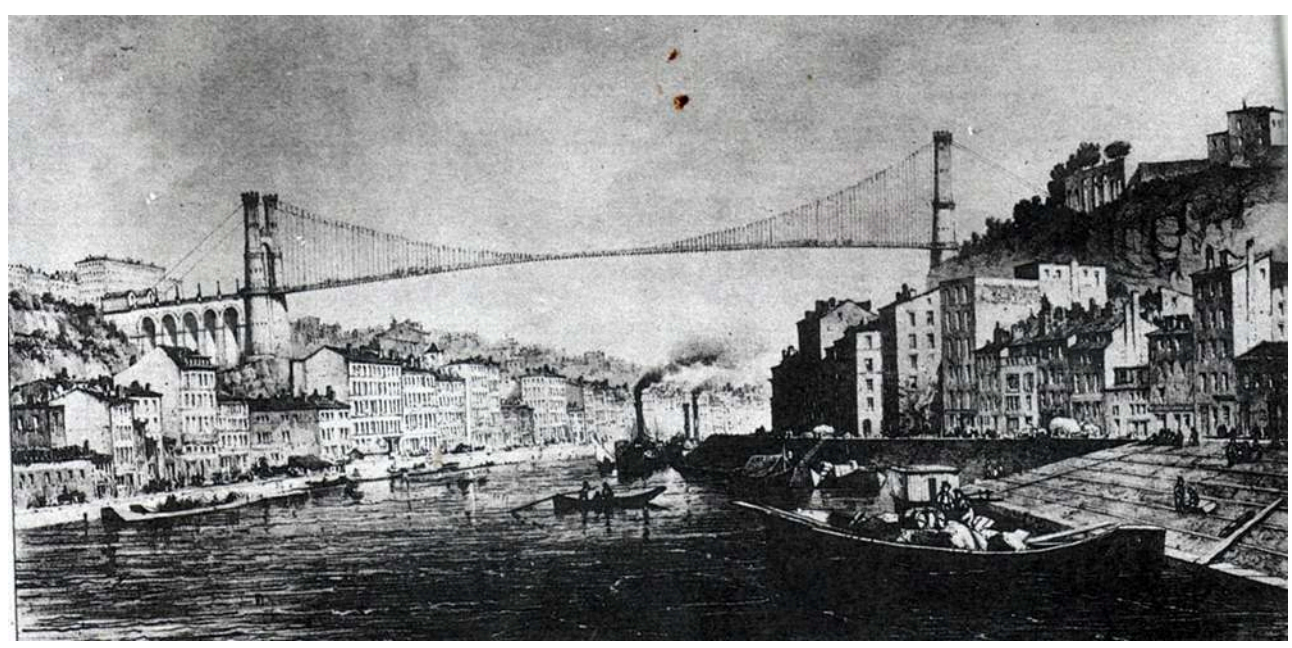

Doc. V. Giorgiutti. 
Fig. 7. - Un pont à treillis.

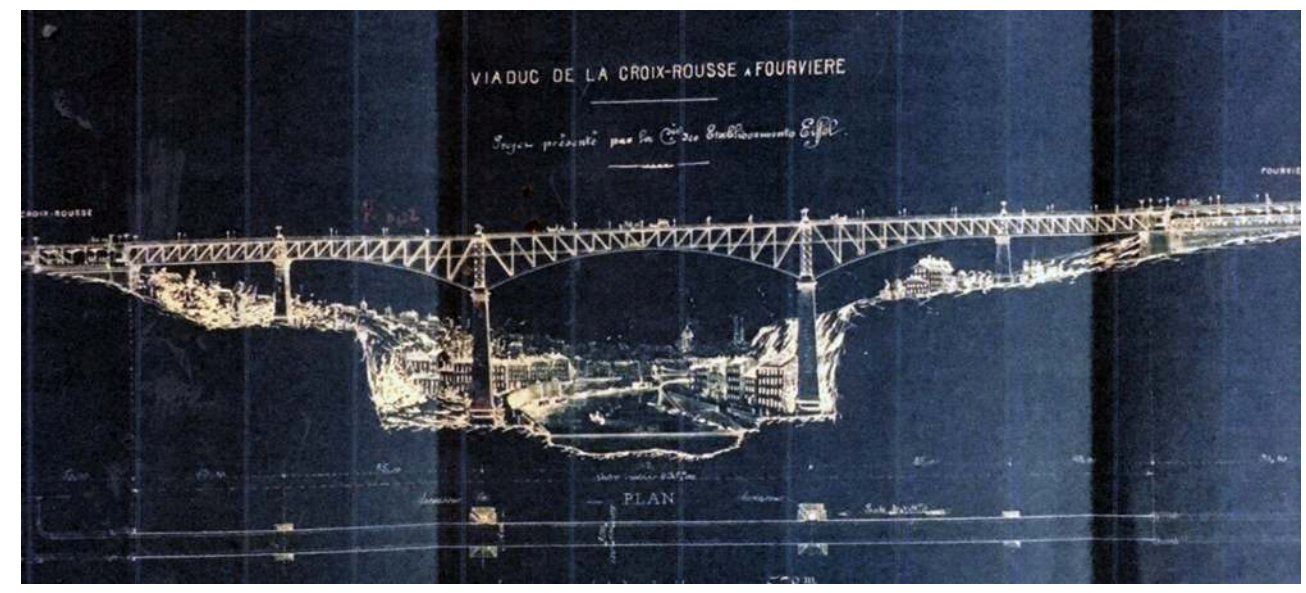

Doc. V. Giorgiutti.

Fig. 8. - Un pont à haubans.

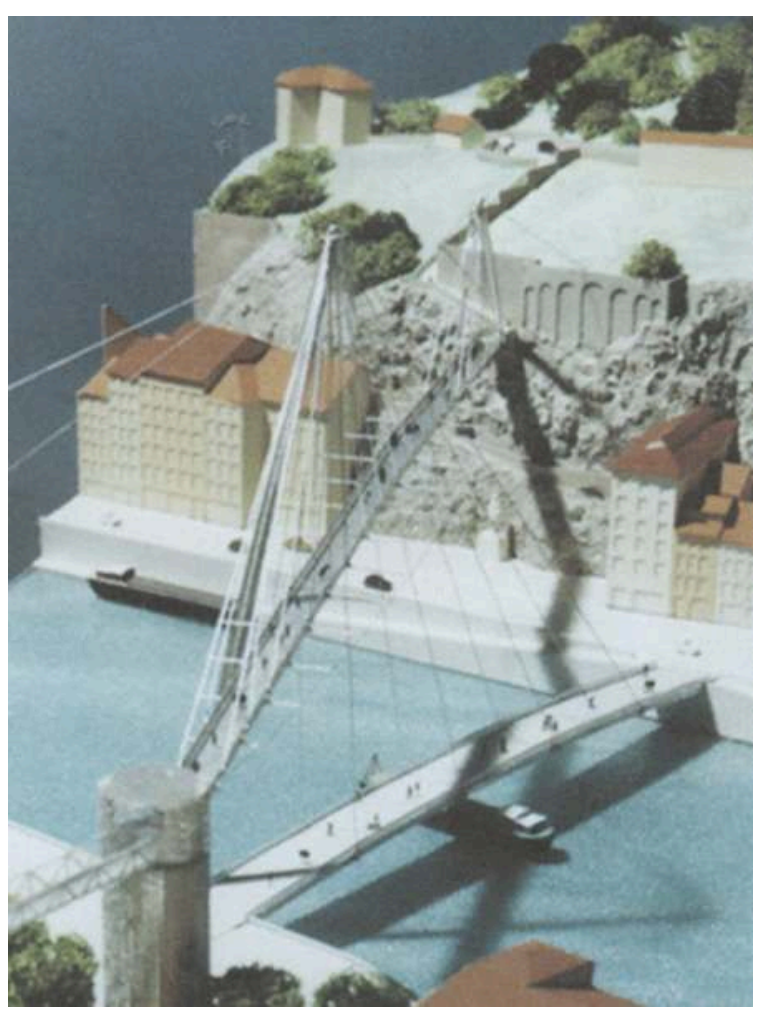

Doc. V. Giorgiutti. 
Fig. 9. - Le pont Chipier à deux étages.

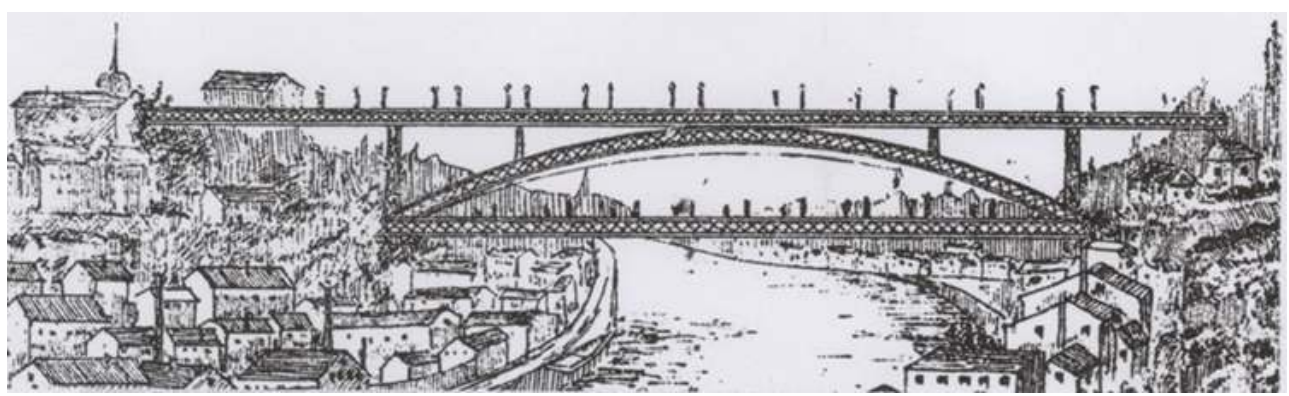

Doc. V. Giorgiutti.

Pour les franchir, les modes de locomotion envisagés sont parfois originaux. L'idée d'un tramway électrique est proposée par Dumont en 1893 et par la compagnie Teste, Pichat, Moret en 1895. Bonnefoy élabore :

" un projet de câble aérien avec nacelle suspendue et mue électriquement ${ }^{26}$.»

La Société des transporteurs continus et universels conçoit :

« Un pont inaccessible aux voitures et aux piétons ordinaires, desservi par une série ininterrompue de véhicules entraînés par un organisme mécanique à mouvement continu $^{27}$. »

4 Les ascenseurs utiles pour atteindre le niveau supérieur apparaissent dans huit projets. Les concepteurs et les décisionnaires ont développé des arguments pour expliquer leurs objectifs dans quinze projets. Trois temps se discernent clairement. Dans le premier, on insiste avant tout sur l'agrément que pourrait procurer ce pont: promenade, beauté des paysages, développement du tourisme. Le gain de temps sur la longueur des trajets, l'avantage militaire pour la défense de la ville avec deux grands champs de manœuvre au-dessus des Chartreux et de la Sarra, le revenu que procurera le péage du pont et la valeur augmentée des terrains ne sont cependant pas négligés. L'aspect urbain est également mentionné : création de nouveaux quartiers dont l'altitude assurera la qualité de l'air ${ }^{28}$.

5 Dans un deuxième temps, l'utilité est mise en avant. En 1886, Moyret pense qu'il pourrait conduire l'eau venue des massifs granitiques de l'est à la Croix-Rousse dans de gros tuyaux, et assurer l'air comprimé nécessaire aux industries du plateau et la nuit de l'éclairage électrique, ce qui retiendrait le commerce et l'industrie et donnerait du travail aux ouvriers ${ }^{29}$. Dès 1872 , Louis Combet explique que son pont de pierre :

«Pourrait servir à l'établissement d'une voie ferrée qui après s'être soudée au chemin de fer de Sathonay desservirait le haut plateau, Saint-Just, Saint-Irénée, Francheville $^{30}$."

Reprenant l'idée de Combet, en 1890, Clavenad montre sa nécessité « pour combler une véritable lacune de notre réseau ferroviaire et le compléter ${ }^{31}$ ». Cette ligne de plaisance servira aussi à l'économie agricole et industrielle notamment pour l'évacuation du charbon. Lyon deviendra ainsi le centre d'un vaste hinterland. Teste fils, Pichat, Moret et Compagnie insistent également sur le développement urbain et économique des deux quartiers. Il faut donc :

« Relier entre eux ces quartiers si salubres et si pittoresques d'abord par une route pour ainsi dire de plein pied et les rattacher ensuite plus étroitement à la basse ville et au réseau général de chemin de fer. La facilité des communications urbaines et l'économie réalisée sur les transports de toute nature secondés par les progrès de l'industrie qui permettent le transport de l'énergie à bon marché sans se 
préoccuper de la situation topographique seraient un puissant élément de régénération qui s'impose aujourd'hui d'une manière plus impérieuse à l'attention de la municipalité ${ }^{32}$." rentabilité de l'opération. Seize autres présentent des dossiers fortement étayés et leur plan de financement. Moyret estime son projet à 80000000 francs; Préaud et Avril le chiffre à 2800000 francs. Teste, Pichat et Moret ainsi que Dumont font un calcul de rentabilité. C'est cependant la proposition de Clavenad qui est la plus informée. Il a sollicité les organismes compétents pour connaître le tonnage de charbon transporté de la Loire à Lyon et le nombre de voyageurs sur les différents secteurs concernés. Il en déduit ce que rapportera la ligne : 3262204 francs, alors que la construction - viaduc métallique, aménagement des routes et expropriations - coûtera 5000000 francs. Il énumère aussi les ouvrages d'art et les souterrains à réaliser. d'affaires, d'un "groupe de financiers de la région lyonnaise ${ }^{38}$ ", de quatre associés «qui ont à leur disposition une maison de banque de Lyon qui se charge de toute l'opération financière ${ }^{39} "$, une société anonyme spécialement fondée, des entreprises Clavenad est en relation avec la société Fives-Lille ${ }^{40}$ et les chemins de fer de l'ouest lyonnais; Dumont avec l'entreprise Schneider. Beaucoup, cependant, s'adressent à la ville pour en obtenir une subvention ou les terrains nécessaires pour la tenue du chantier. Le plus souvent, les demandeurs déclarent prendre les frais à leur charge à condition que la municipalité leur confie pour 50 à 99 ans la levée du péage du pont dont on fixe les prix. Certains réclament deux avantages : subvention et péage pendant 60 ans, 600000 francs à verser par annuités et 99 ans, terrains et péage, voire trois (subvention, terrains et 75 ans de péage). À la fin du $\mathrm{xIX}^{\mathrm{e}}$ siècle, ils sollicitent des 
garanties d'intérêt: 108000 francs pendant 60 ans, 700000 par la ville et le département durant 99 ans et 2155000 francs pendant 40 ans.

$\mathrm{Au}$ surplus, les candidats doivent effectuer des démarches et remplir certaines conditions juridiques comme en 1847, fournir «des documents exigés par les ordonnances royales du 18 février 1834 et 23 août 1835 ». Les projets sont d'abord envoyés, accompagnés d'un courrier à l'autorité qui dirige la ville. Dans onze cas, il s'agit du maire. Dans dix autres, entre 1852 et 1867, de 1867 à 1870 et de 1873 à 1881 , la ville étant divisée en cinq puis six arrondissements administrés par le préfet du Rhône, au préfet. En 1852 et en 1869, Bourget et Combet préfèrent s'adresser au princeprésident devenu empereur. De toute manière cependant, le cheminement du courrier est identique: dix-huit projets sont envoyés à l'ingénieur de la ville; trois à une commission municipale, du conseil général (Eiffel) ou préfectorale (Vilbien). Pour quatorze projets, l'ingénieur rend rapidement son avis : de quatre à seize jours pour six ; de un à quatre mois pour huit. Le verdict tombe au bout d'un an pour sept. Berchet est fixé après quatre ans d'attente et Clavenad au bout de six. Au bout du compte, aucun n'est réalisé.

\section{Expliquer les échecs}

Les raisons des échecs sont inconnues pour vingt d'entre eux. Pour les autres, elles sont variées. En dehors de Vilbien pour lequel une commission se prononce, le refus est le fait de l'ingénieur qui en informe le maire ou le préfet. Dans trois cas, les projets sont jugés insuffisants.

Le plus souvent, cependant, l'ingénieur examine le projet très attentivement avant de le rejeter pour quatre raisons. Pour cinq d'entre eux, ce sont des impossibilités techniques qui sont relevées: ainsi pour les ponts consoles ou les ponts suspendus. Pour deux, c'est la faiblesse des calculs de coût et de rémunération qui est mise en cause. Pour six, l'ingénieur soulève le problème du financement: ainsi en 1872 à Combet et en 1875 à Givord et Raclet.

En quatrième lieu, les ingénieurs montrent le décalage existant entre le projet envisagé et l'évolution de la situation : en 1865, on répond à Drevet et à Vettard qu' « il existe d'autres moyens faciles de mettre en rapide communication les deux collines » et à Givord et Raclet que leur projet «a perdu de son opportunité ». En 1893, Fabrègue vante les mérites des funiculaires ${ }^{41}$ et en 1903 :

«Ceux des tramways pénétration Perrache-Croix-Rousse, place du pont CroixRousse qui ont une influence infiniment plus efficace car ils mettent le plateau en communication avec le centre des affaires et le cœur de la ville alors que le viaduc au contraire établirait un lien entre deux faubourgs de temps immémorial qui n'en ont jamais eu et n'ont jamais songé à en avoir et l'on sait combien il est difficile de vaincre des habitudes invétérées ${ }^{42}$. "

Enfin, les circonstances et les aléas de la vie politique peuvent expliquer les échecs de deux projets. En 1847, en dépit du problème des voies d'accès au pont, Lehaitre obtient le feu vert de la commission préfectorale. Survient la Révolution de 1848: les travaux commencent sur la colline de la Croix-Rousse grâce aux ateliers nationaux. Dans les deux années suivantes, une enquête est réalisée en vue des expropriations nécessaires. En 1852, Lehaître rappelle son projet mais le préfet a changé, et la mairie lui fait savoir qu'aussi bien elle que l'État ne sont pas «dans l'intention de continuer les travaux du 
cours de Fourvière ». En 1890, le projet Clavenad franchit tous les obstacles. "La population lyonnaise tout entière si froide d'ordinaire s'est passionnée pour cette œuvre ». Une grande partie de la presse le soutient. Le prolongement vers SaintÉtienne de la ligne Lyon Saint-Just à Vaugneray et Mornant est examiné par le Conseil général de la Loire. Le 24 mars, le préfet reçoit le traité préparé en début d'année entre la compagnie Fives-Lille et la compagnie de l'Ouest lyonnais fixant les objectifs ferroviaires et les conditions financières du projet, notamment la garantie pendant 90 ans par la ville et le département de l'intérêt annuel d'un capital de 14000000 francs (700 000 francs). Le 11 avril le conseil municipal, dans une salle comble, se déclare favorable au projet. Le Conseil général l'approuve le 25 . Le 21 juin, l'enquête nécessaire à la déclaration d'utilité publique est annoncée par voie d'affiche et se déroule sans difficultés entre le 23 juin et le 24 juillet. La même année, le Conseil général accorde une avance de 200000 francs remboursable sans intérêts à la compagnie des chemins de fer de l'Ouest. Le 20 avril 1892, la convention signée entre la compagnie de l'Ouest lyonnais et le Conseil général de la Loire parvient au préfet du Rhône qui la transmet au Conseil général. Après approbation, ce dernier l'adresse au Conseil municipal de Lyon qui ne fait rien. Le 8 février 1893, les ministres des Travaux publics et de l'Intérieur déclarent que l'avance de 200000 francs est une pure libéralité et la refusent. Le 4 novembre 1895, Lyon fait enfin connaître sa réponse sur la convention transmise trois ans plus tôt :

"Considérant que les charges financières que lui impose le projet de traité sont sans compensation et d'une importance trop lourde et d'une durée trop longue pour les ressources de la ville, mais considérant que la ligne est d'une utilité incontestable ; que cette utilité est d'ordre général ; qu'il y a lieu d'émettre le vœu qu'elle soit exécutée sur l'initiative et par les soins de l'État, délibère $: 1^{\circ}$ est rejeté le projet de traité présenté par les compagnies Fives-Lille et de l'Ouest lyonnais $2^{\circ}$ émet le vœu que l'État prenne à sa charge ou impose à la compagnie PLM l'exécution de cette ligne ${ }^{43}$.»

Le Conseil général suit le 29 avril 1896. Ainsi prend fin le projet Clavenad en dépit de pétitions diverses. Sans doute faut-il voir dans ce revirement les effets des luttes d'influence entre le maire Gailleton et le conseiller Augagneur, très hostile au projet dès le début et l'effet de la décision prise, cette même année, de construire le pont de l'Homme de la Roche unissant les deux rives de la rivière.

En près d'un siècle et demi, le projet d'union des deux collines lyonnaises n'a donc pas été concrétisé. Faut-il comme Moyret, incriminer le caractère «discutailleur » de la municipalité lyonnaise : «à Lyon on discute beaucoup mais on ne fait rien » ou comme Clavenad, sa pingrerie : «la municipalité lyonnaise est essentiellement économe, trop économe même parfois ». N'est-ce pas plutôt son bon sens qu'Ernest Fabrègue souligne :

«Les conséquences sur tous les esprits un peu chimériques doivent être incalculables ? ${ }^{44} »$

Cependant, cette utopie s'est maintenue jusqu'à nos jours, certes avec moins de frénésie qu'autrefois, jusqu'à faire l'objet d'études à l'École nationale d'architecture de Lyon et d'un concours de la défunte Communauté urbaine de Lyon mobilisant les grands noms de l'architecture européenne. Car en 1907, l'idée n'est que repoussée : 
« Il est possible qu'un grand viaduc s'impose plus tard ${ }^{45}$. » Mais pour l'instant, comme le disait Moyret :

«Les centenaires en ont entendu parler en suçant le lait de leurs nourrices ${ }^{46}$. »

\section{BIBLIOGRAPHIE}

GIORGIUTTI Véronique, Un pont entre deux collines, 45 projets pour un pont reliant la Croix-Rousse à Fourvière, Lyon, CAUE, s. d.. PELLETIER Jean, Ponts et quais de Lyon, Éditions lyonnaises d'art et d'histoire, Lyon, 2002. PELLETIER Jean, Les ponts de Lyon, L'eau et les Lyonnais, Horvath, Roanne, 1989. POUSSE Jean-François, Jourda et Perraudin, I.F.A., Liège, P. Mardaga, 1993. PREST Véronique, « Jourda et Perraudin, écologie et haute technologie », Art Presse, $\mathrm{n}^{\circ}$ 180, mai 1993, p. 46-48.

\section{NOTES}

1. J. Pelletier, L'eau et les Lyonnais, les ponts de Lyon, p. 73.

2. J. Pelletier, Ponts et quais de Lyon, p. 7-10.

3. Le résultat est cependant assez maigre : la COURLY (Communauté urbaine de Lyon ou Grand Lyon), initiatrice d'un concours en 1990, n'existe plus. La métropole qui lui a succédé n'a pas versé les archives aux archives départementales et métropolitaines du Rhône et d'ailleurs, elle ne conserve pas les documents d'un concours infructueux plus de dix ans.

4. Arch. mun. de Lyon 342 WP 16, 23 septembre 1852 ; 342 WP 16, 26 août 1875.

5. Ibid., 342 WP 16, dossier 17, 24 juillet 1875. G. Eiffel a fondé sa société basée à Levallois-Perret en 1866 ; 342 WP 17, 31 octobre 1892. La société en commandite simple Schneider et Compagnie est spécialisée depuis 1845, au Creusot, dans l'acier, les chemins de fer l'armement et la construction navale; 342 WP 17, 15 juin 1895. L'association Teste père et fils et Moret date de 1885. Cette puissante société fabrique des articles de mercerie et des gros câbles porteurs avant de se lancer en 1897 dans la fabrication d'automobiles.

6. Dont Trévoux (ibid., 342 WP 16, 19 juillet 1871) ; Lehaître (342 WP 16, $1^{\text {er }}$ juin 1847) ; Dumont (937 WP 102, 28 janvier 1891) ; Moyret (Arch. dép. et métrop. du Rhône PER 429/1, 20 juillet 1886).

7. Arch. mun. de Lyon 342 WP 16, 2 août 1853 ; 512 WP 15/2, 14 juin 1897.

8. Ibid., 512 WP 15/2, 14 juin 1897.

9. Ibid., 342 WP 16, 11 juillet 1852 ; 1612 WP 241/1, 13 août 1869; 342 WP 1627, février 1869. 
10. Ibid., 342 WP 16, 29 mars 1858.

11. Ibid., 342 WP 16, 4 mars 1865 ; 18 novembre 1865 ; 342 WP 17, 28 janvier 1891.

12. Ibid., 342 WP 16, dossier 18.

13. Ibid., 342 WP 16, dossier 16, 9 avril 1875.

14. Ibid., 937 WP 103, « Revue lyonnaise illustrée », $n^{\circ} 29,1890$.

15. Ibid., 937 WP 102, 8 juin 1896.

16. Ibid., 342 WP 17, 9 mars 1903.

17. Ibid., 342 WP 17, dossier 18, 10 septembre 1890.

18. Ibid., 937 WP 103, coupures de presse, 26 septembre 1891 ; n 51 Courrier de Lyon, 27 août 1890.

19. Ibid., 937 WP 103, 21 avril 1890.

20. Ibid., 937 WP 103, n 56, 26 octobre 1890.

21. Ibid., 342 WP 17, 1893.

22. Pont dont le tablier est constitué de poutres construites en porte-à-faux. On dit aussi « par encorbellements successifs ».

23. Arch. mun. de Lyon, 342 WP 16, 18 novembre 1865.

24. Un pont métallique est un pont dont la structure est réalisée en métal, à savoir en fer, en fonte ou en acier.

25. Dans un pont à haubans, le tablier est suspendu par des câbles eux-mêmes soutenus par des pylônes.

26. Arch. mun. Lyon, 512 WP 15/2, 18 juin 1896.

27. Ibid., 342 WP 17, 3 avril 1895.

28. Ibid., 342 WP 16, 2 mars 1848.

29. Arch. dép. et métro. Rhône, PER 429, Journal l'indépendance politique, financier, industriel, commercial, économique, 16 mai 1886.

30. Ibid., 342 WP 16, dossier 12, 2 novembre 1872.

31. Arch. mun. Lyon, 937 WP 102, 22 février 1890.

32. Arch. mun. de Lyon, 342 WP 17, 15 juin 1895.

33. Ibid., 1217 WP 204, p. 682, 15 avril 1946.

34. V. Giorgiutti, Un pont entre deux collines, p. 22.

35. P. Goulet, dans J.F.Pousse, Jourda et Perraudin, p. 6.

36. V. Prest, « Jourda et Perraudin, écologie et haute technologie », p. 47.

37. V. Giorgiutti, p. 20.

38. Arch. mun. de Lyon 342 WP 16, $1^{\text {er }}$ juin $1847 ; 2$ août 1852.

39. Ibid, 342 WP 17, 28 janvier 1851.

40. La société anonyme compagnie de Fives-Lille spécialisée dans le matériel ferroviaire s'est créée en 1868. Elle possédait une usine à Oullins spécialisée dans les charpentes métalliques, les ponts en fer et le matériel de guerre.

41. Le premier funiculaire a relié Lyon à la Croix-Rousse en juin 1862. Le funiculaire Lyon Saint-Just a été créé en 1886.

42. Arch. mun. Lyon, 937 WP 102, 15 avril 1907. 
43. Arch. mun. de Lyon 342 WP 017/014, 4 novembre 1895 : Arch. dép. et métro. Rhône $1 \mathrm{~N} 167$ p. 549.

44. Arch. mun. de Lyon 342 WP 017, 15 avril 1907.

45. Ibidem.

46. Arch. dép. et métro. Rhône, PER 429, 1886-1887.

\section{RÉSUMÉS}

Entre 1847 et 1991, 44 projets d'un pont reliant les collines de Fourvière et de la Croix-Rousse ont été réalisés. Les archives municipales de Lyon, départementales et de la métropole du Rhône et les journaux conservent des dossiers sur la plupart d'entre eux. Ils comprennent le nom et la qualité de leurs auteurs, leurs descriptions ou leurs plans, les matériaux utilisés, les travaux à réaliser, leurs prix, leurs objectifs ainsi que les avis des contemporains, le tout évoluant au fil du temps, de l'avancée des techniques et des conceptions de l'urbanisme. Pour des raisons diverses qu'on peut retrouver parfois dans les délibérations du conseil municipal, aucun d'entre eux n'a été construit, restant ainsi à l'état de documents virtuels.

\section{AUTEUR}

\section{FRANÇOISE BAYARD}

Professeur honoraire d'histoire moderne, Université Lumière - Lyon 2 
Voyage réel, quête virtuelle 


\title{
Entre réel et imaginaire, un monde de synthèse : les voyages de Vincent Leblanc, Marseillais
}

\author{
Henri Bresc
}

1 Les mémoires de Vincent Leblanc, voyageur marseillais (vers 1553-vers 1635) ont suscité l'intérêt du grand érudit Nicolas-Claude Fabri de Peiresc qui a déployé ses efforts pour en donner une édition ${ }^{1}$. En 1619, Peiresc confie à Pierre Bergeron, avocat parisien, de les simplifier et d'en éliminer les passages sur la « planitude » de la terre 2 . Vincent Leblanc, résiste, cherche à contourner son patronage et publie à Aix en 1634 une Histoire géographique et mémoriale de l'assiette de la terre/universelle laquelle est comprise en une juste émisphère, ensemble les voyages et/Pérégrinations de Vincent Blanc de Marseille faictes aux quatres parties du monde, saisie à la demande d'un officier de l'ordre de Malte.

2 En 1648, le jésuite Louis Coulon édite enfin à Paris l'ouvrage préparé par Bergeron, décédé en 1640. Les Voyages fameux du sieur Vincent Leblanc marseillais qu'il a fait, depuis l'âge de douze ans jusques à soixante, aux quatre parties du monde sont aussitôt un succès éditorial, réédité en 1649 , en 1658 , en 1664 , traduit en néerlandais dès 1654 , et en anglais en 1660. Cinq volumes de manuscrits sont conservés à la Bibliothèque nationale de France ${ }^{3}, 2143$ pages au total, dont une petite moitié consacrées à la polémique contre la rotondité de la terre, qui contiennent 182 dessins dont 79 « portraits » de villes, six cartes et sept mappemondes, quelques images et des figures astronomiques.

Les Voyages ont suscité de nombreuses études fondées sur l'édition de Bergeron et Coulon. Maurice Laugaa avait perçu la nécessité d'un décorticage des manuscrits, vrais «travaux d'Hercule" », et Grégoire Holtz a fait des plongées dans le matériau original 5 . Le travail sur les manuscrits a permis d'éclairer un aller-retour entre réel et imaginaire créateur d'un monde entre vrai et faux, une "Indie» unifiée, qui va du détroit de Magellan jusqu'à Bornéo et un ensemble de royaumes utopiques. Les deux versions ne rapportent pas seulement le récit d'un voyage réel auquel s'agrègent des excursions dans l'imaginaire, mais un voyage extraordinaire ${ }^{6}$ qui place dans un temps et dans un espace réels des sociétés imaginaires, des "allotopies» et une "allochronie». Ce 
monde virtuel flotte encore, comme l'île de Gulliver, dans la géographie du XVIII ${ }^{e}$ et du début du xIX siècle.

\section{Vie et voyages de Vincent Leblanc}

4 Marchand, marin, mercenaire, joaillier autodidacte, Vincent Leblanc appartient au milieu du consulat des marchands. Son père, Rafael Bianco, page de Cosme de Médicis, a fui Florence pour Constantinople après un meurtre, puis s'est fixé à Marseille comme armateur. Vincent Leblanc quitte Marseille en 1567, à onze ans selon lui, quatorze plus vraisemblablement, porté par une ardente curiosité, pour « soixante ans de voyages ».

Il a appris un peu de latin, le français, et le dessin. Il dessinera sur « un livret des lieus où nous passions crayoné grosièrement » des vignettes qui servent de preuve. Il cite des auteurs latins et ses manuscrits conservent huit poèmes, sonnets et quatrains, dont l'orthographe, calque du provençal, contraste avec la bonne prosodie ; il cite aussi des poèmes en castillan, dont l'Araucania. Polyglotte, il a appris l'arabe au Caire, le grec à La Canée, le portugais et le castillan, et un peu de malais.

\section{Le grand voyage de jeunesse aux Indes}

Son premier voyage le mène au Caire. Sur le chemin du retour, le navire fait naufrage au large de la Crète. Recueilli à La Canée, il fait vœu de pèlerinage au Saint-Sépulcre ; arrivé de Marseille, Guilhem de Cassis, pilote et marchand, le conduit en août 1568 près de Damas chez son frère, renégat, et de là en Arabie, par Médine et La Mecque, dont la description est empruntée au voyageur Varthema. Du Yémen, ils passent à Ormuz, visitent l'Iran, pour retourner en Arabie et, d'Aden, gagner Calicut en mai 1570 (fig. 1).

Fig. 1. - Le premier voyage, 1567-1576, l'Arabie.

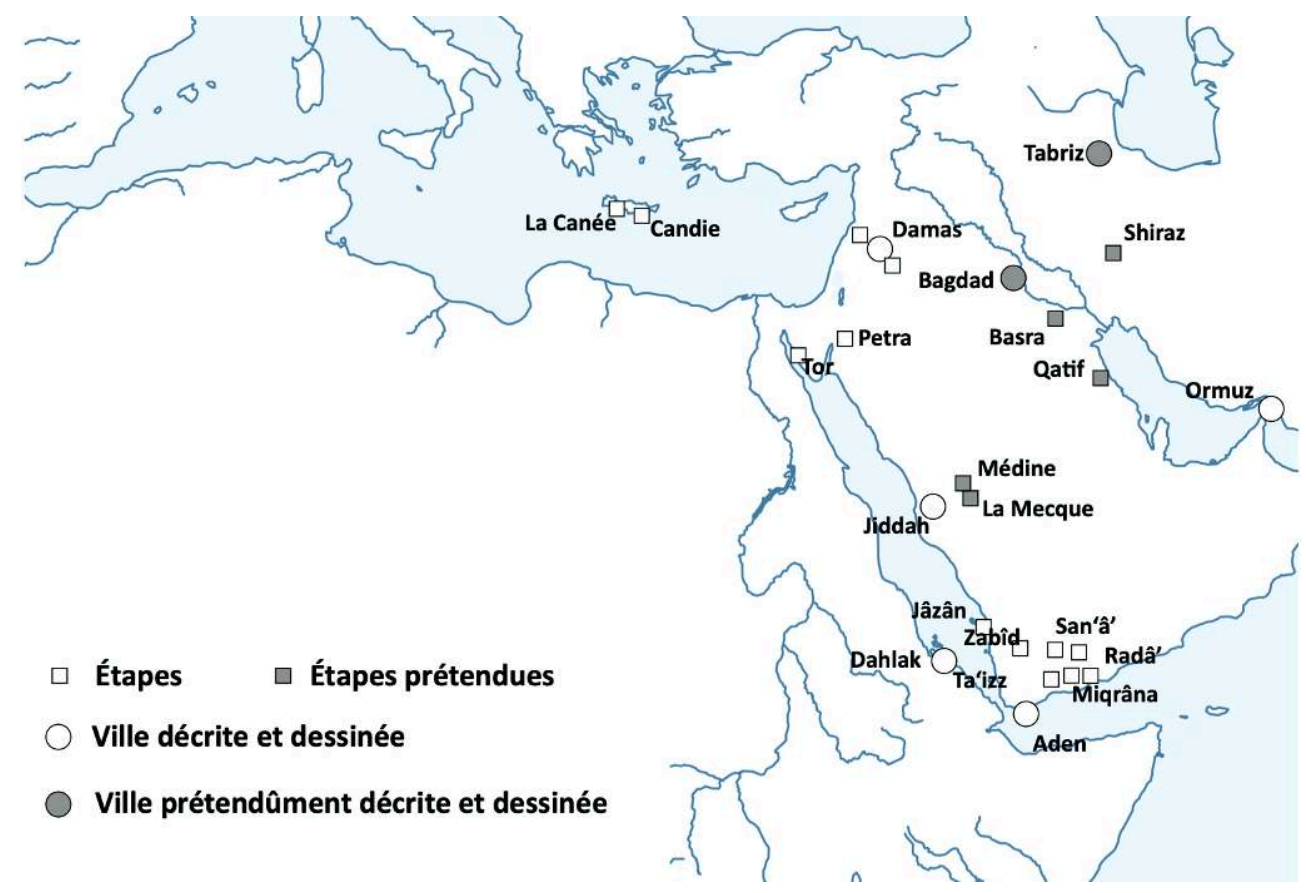


Son séjour aux Indes dure quatre ou six ans, de 1570 à 1575, selon les manuscrits. Bergeron reconstitue un itinéraire qui suit le littoral du Gudjerat jusqu'à Sumatra. Les récits autographes indiquent au contraire des allers-retours sur la côte de l'Inde méridionale, puis de Calicut, une traversée directe vers le Pégou en 1571, pour un long séjour et des visites dans les provinces environnantes, à Pidir (Sumatra) en 1573, au Siam, en Transiane (principauté thaïe de Tranh Ninh). De Pégou, il entame son retour par Cambaya et Diu (fig. 2). Son récit s'appuie sur de nombreux voyageurs, Marco Polo, Odoric de Pordenone, Nicola de' Conti, Varthéma, Barbosa, Mendes Pinto, Federici, Balbi, Pyrard et Andrade. Et un segment d'itinéraire, de Pégou à Catigang (Chittagong), copié sur Federici, en reprend les éléments pittoresques, typhon, échouage, refuge sur une île.

Fig. 2. - Le premier voyage, 1567-1576, les Indes.

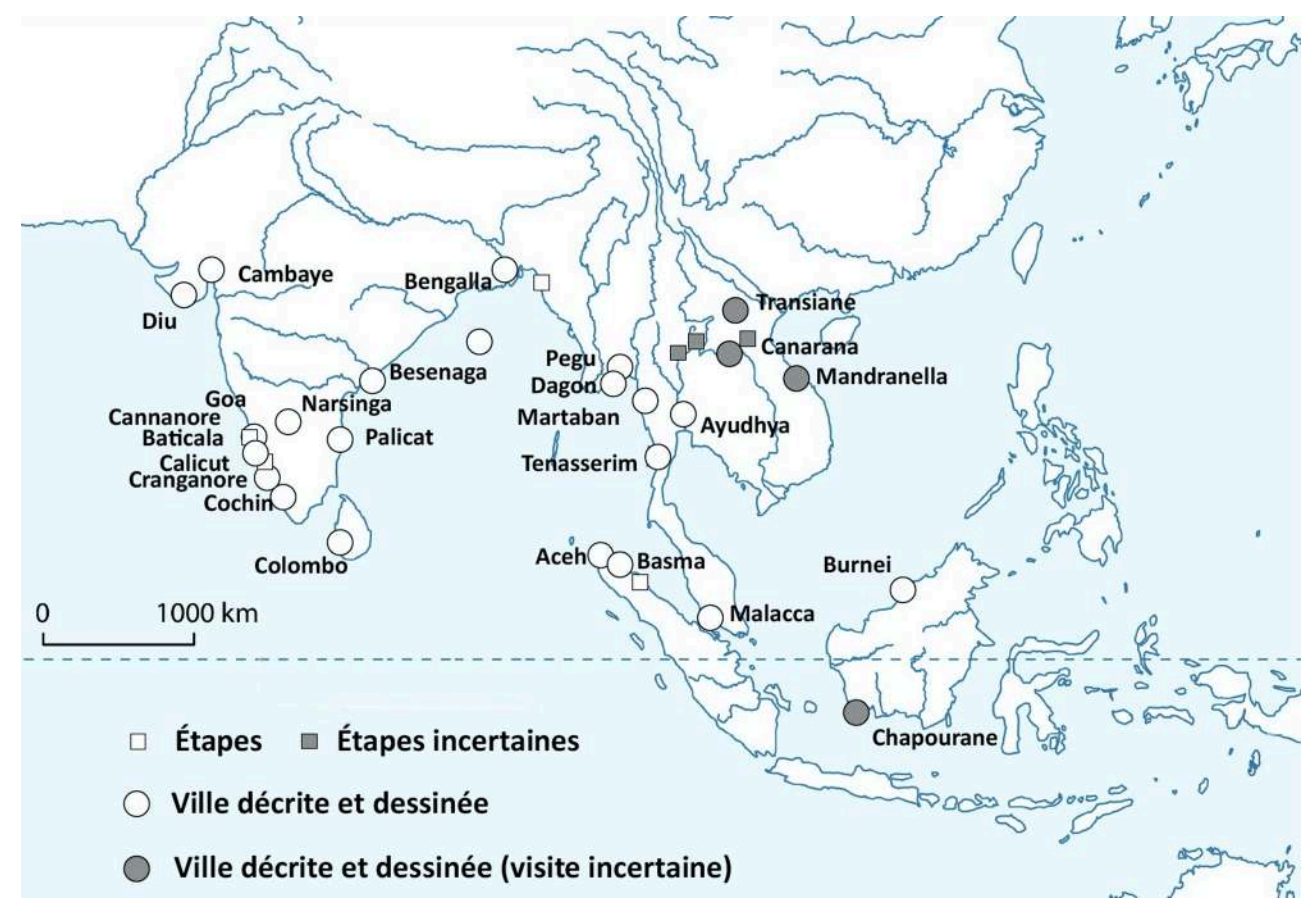

Doc. H. Bresc.

Son retour l'aurait mené à Madagascar, dont il donne une géographie imaginaire, puis au Mozambique et à Sofala, dans la ville chimérique de Madrogan, et au Monomotapa, à la richesse fabuleuse, royaume de l'or et des citadelles de pierre cyclopéennes. Emmenés depuis Pégou par le sieur de la Courbe, Vincent Leblanc et ses compagnons auraient alors entamé une traversée héroïque de l'Afrique, remontant le Zuama/ Zambèze jusqu'au lac central de l'Afrique, source du Nil, puis entreprenant la descente du fleuve avec deux nacelles, dont la plus grande aurait porté un éléphant, par des défilés dangereux (mais le récit ignore les cataractes) et des passages en basses eaux jusqu'aux premiers monastères éthiopiens.

« Nous n'avions mis que huit mois pour traverser l'Afrique ${ }^{7}$. "

Outre Jean Léon l'Africain, les sources citées, ou inavouées, sont portugaises, Barros, Dos Santos, Alvares, Bermudes, Osorio et Godinho, tandis que l'itinéraire repose sur la carte d'Ortelius qui place les lacs et les villes d'Éthiopie dans la région des Grands Lacs. 
En Éthiopie, les épisodes imaginaires (de La Courbe au Négus, guérison du roi de Seiton soigné par La Courbe, défilé de l'armée du Négus et saison à la Cour, avec théâtre et musique) se greffent sur les récits des ambassadeurs et missionnaires portugais. Un séjour est cependant probable, à partir de la mer Rouge et du port de Suwakin, à Baghaméder dans la région du lac Tana dont il décrit les églises taillées dans le roc et les monastères.

11 D'Éthiopie, les voyageurs sont censés descendre le Nil vers la Nubie, d'où, en cinq jours, ils auraient gagné Le Caire, par Soba, Dongola et une multitude de villes égyptiennes. Du Caire, en 1576, Vincent Leblanc rentre, par Tripoli et Malte, à Marseille.

Ce premier voyage est celui de la formation de Vincent Leblanc à la joaillerie et au commerce, dans la boutique que les Français tiennent à Pégou le Neuf. Le contact avec les jésuites, dont le P. André de Saint-Paul, apporte une foule d'informations et un point de vue critique sur les religions indiennes (fig. 3).

Fig. 3. - Le premier voyage, le chemin du retour, 1576.

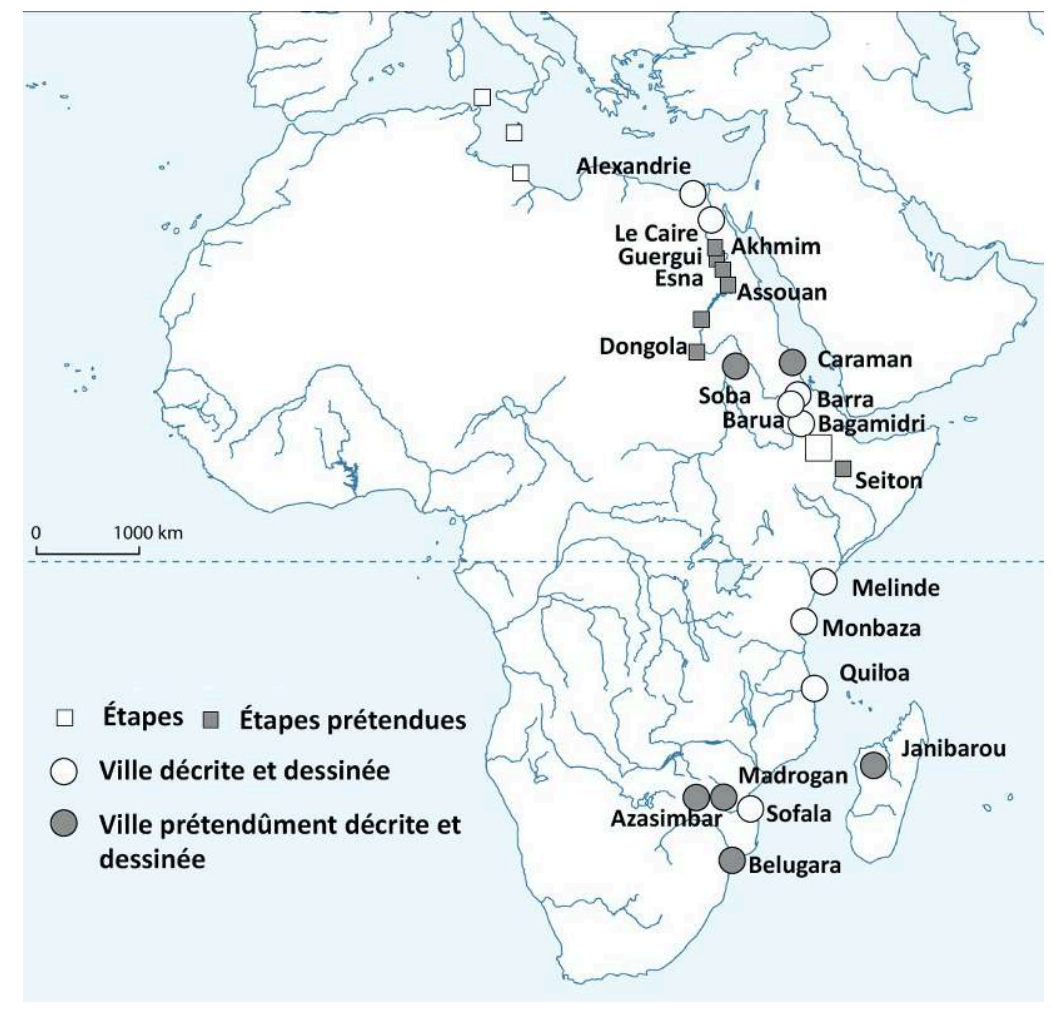

Doc. H. Bresc.

\section{Les voyages picaresques de la maturité}

13 Le deuxième voyage est celui des incidents dramatiques : après six mois de repos, en 1576-1577, Vincent Leblanc s'ennuie. Il décide d'accompagner un ami de son père, ambassadeur du sultan de Maroc Mulay ‘Abd al-Malik à Henri III et chargé de porter des munitions au sultan. Interceptés à Gibraltar, condamnés à mort pour contrebande, les membres de l'expédition sont graciés sur l'intervention de l'infante Isabelle de la Paix. Un autre incident et une autre condamnation à mort attendent Vincent Leblanc à Meknès - il a uriné sur une tombe sainte -, et un autre pardon. Il assiste enfin à la 
bataille d'Alcazarquivir, qu'il raconte en détail (4 août 1578) et revient par Cadix, Séville, Grenade, Cordoue, Carthagène, Valence et Barcelone (fig. 4).

Fig. 4. - Les voyages picaresques de la maturité, 1577-1592.

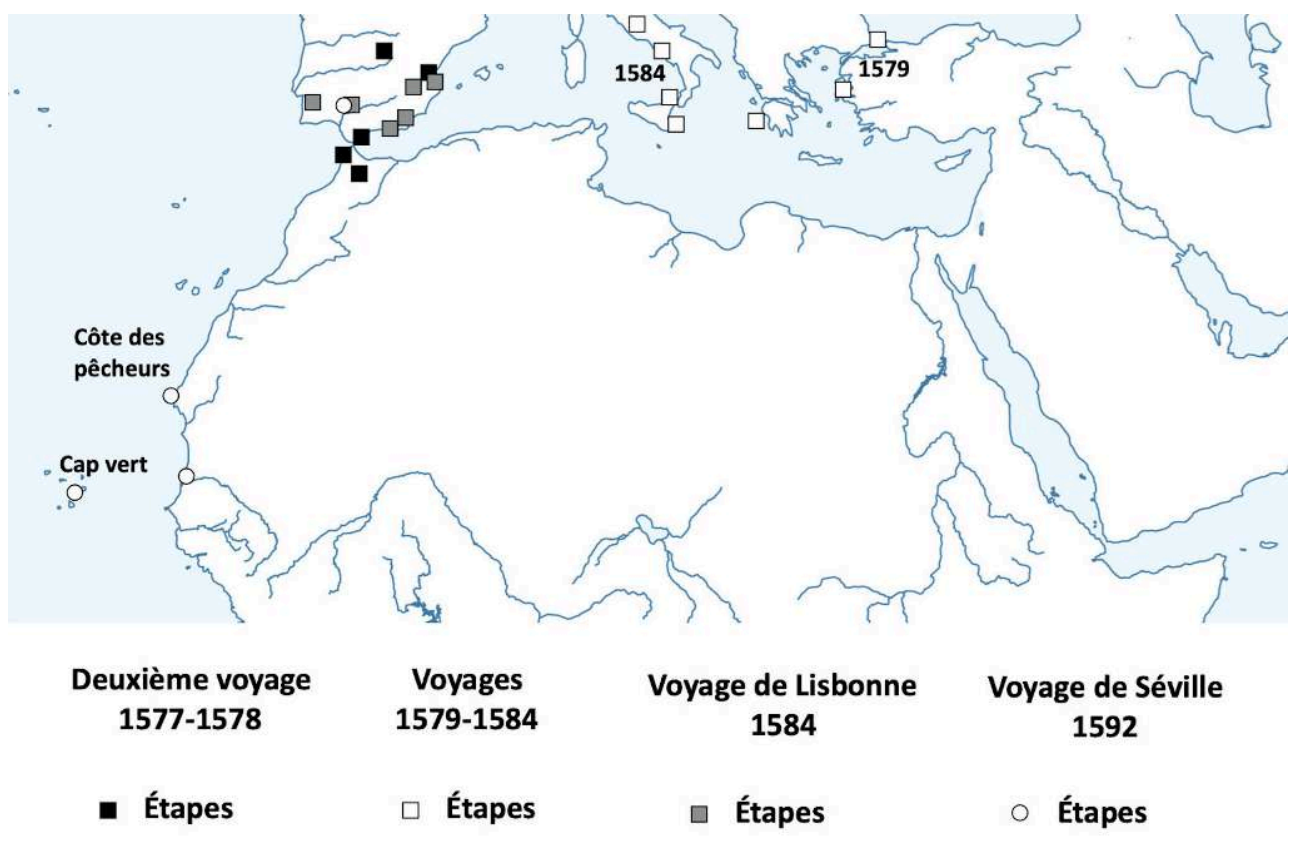

Doc. H. Bresc.

Plusieurs voyages rythment les années suivantes, et d'abord une brève expédition de trois mois à Constantinople, ville belle et sale, où un renégat marseillais, Benjamin Nadal, l'informe sur le sérail, le gouvernement de la "petite fenêtre » et sa justice rigoureuse. À son retour, il repart visiter l'Italie, Peccioli en Toscane, foyer de sa famille, Florence, Rome, L'Aquila, jusqu'à Naples. Enfin, en 1580, venu à Paris, il participe au siège de La Fère (20 juin-31 août), où il est blessé ; guéri, il accompagne le duc d'Anjou dans la guerre de Flandres. Il manque y perdre une jambe de froid. Malade, il pense se faire capucin, puis rentre à Marseille au temps de la peste et s'y marie avec une femme qui se révèle terrible, un « satan ».

Ces mauvaises relations conjugales le conduisent à partir pour le Brésil dès 1583 sur la Salamandre du Havre, pour un voyage marqué par la famine. L'année suivante, il porte des marchandises à Cadix et à Lisbonne; volé à son passage à Gandia, il se venge en frappant un chanoine et doit aller chercher l'absolution à Rome.

En 1592, enfin, il se rend à Séville, comme « négociant de pierreries et perles »; à Cadix, il entre en société avec des Marseillais pour aller acheter du poisson au cap Blanc (Arguin), sur la Côte des pêcheurs. C'est le "voyage de Guinée », daté avec précision, par le Cap de Non, jusqu'à l'embouchure du Sénégal, à $13^{\circ}$ de latitude.

\section{Le dernier grand voyage}

En 1597 enfin, Vincent Leblanc, âgé de plus de quarante ans, entreprend un grand voyage aux Indes occidentales (fig. 5) : de Marseille à Cadix, puis de Puerto Santa Maria jusqu'aux Antilles par les Canaries, la Désirade, Saint-Domingue - s'y greffe une description des Taïnos et des quatre royaumes de Haïti vers 1492 -, la Navasse et la 
Jamaïque, où un ouragan cause le naufrage et le jet des marchandises que Vincent Leblanc porte en Amérique, enfin Campeche, San Juan de Uloa, jusqu'à Vera Cruz. Des visites assurées rythment le voyage, aux Canaries, à Saint-Domingue, à Cuba, et une autre, très probable, au Mexique, où Vincent Leblanc enregistre le refrain cucurucu qui sera repris au XIX ${ }^{e}$ siècle dans la chanson La Paloma. D'autres sont invraisemblables, en particulier au Nouveau-Mexique: Robert Lindsay y a noté l'absence de "vignettes", scènes ou croquis qui authentifient le récit, et en a conclu au voyage en chambre. Vincent Leblanc et ses éditeurs citent en effet le licencié Ayllon, Pierre Martyr d'Anghiera, Cartier, Benzoni et Acosta et s'inspirent de ces sources.

Fig. 5. - Le voyage aux Indes occidentales, 1597, l'Amérique du Nord.

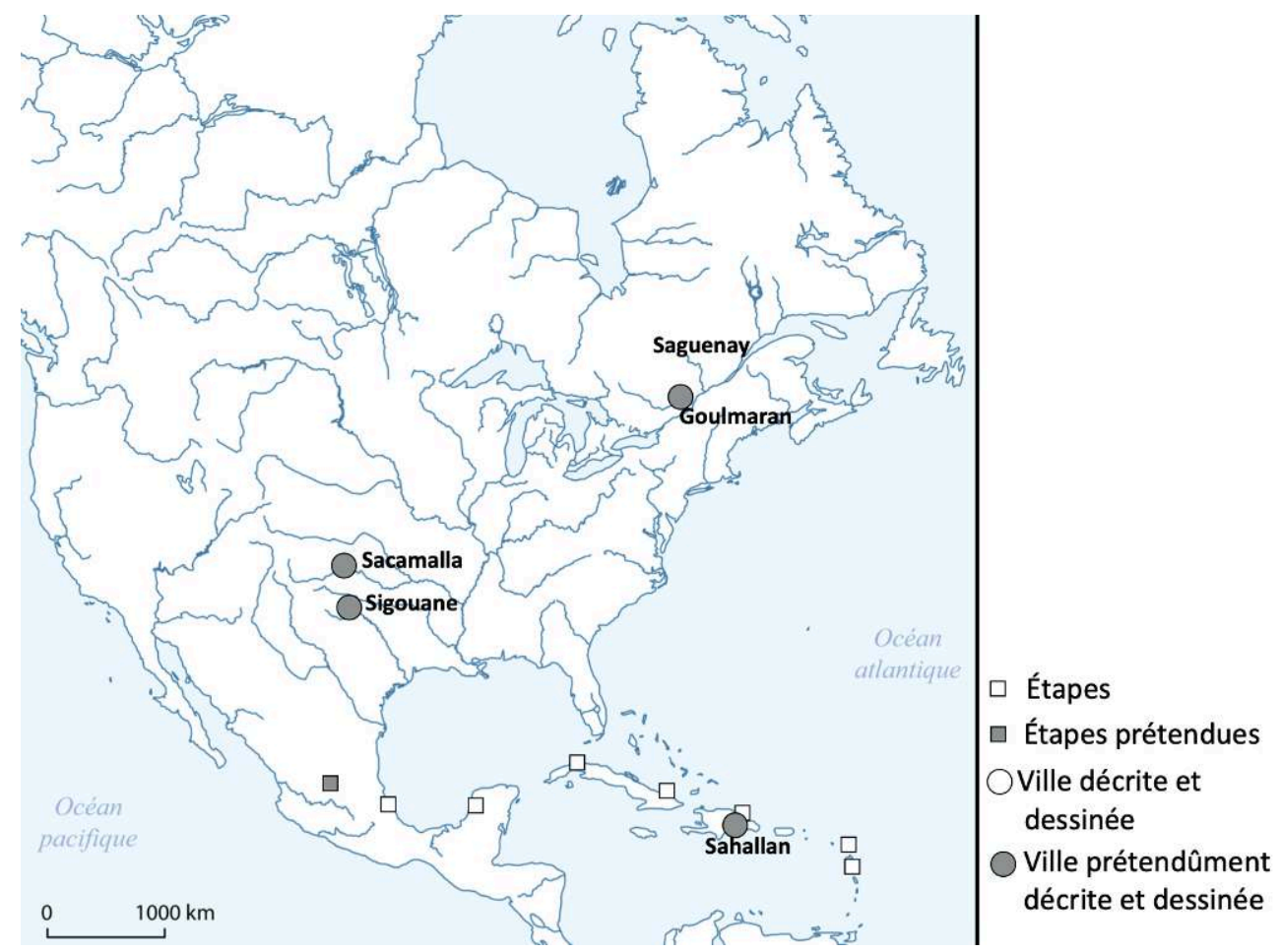

Doc. H. Bresc.

Vincent Leblanc évoque ensuite la Nouvelle Espagne, Lima, puis des pays sur une diagonale entre monde andin et Brésil, Couran, Tabin, Seiton, Caramel (fig. 6). Il copie peut-être une relation inédite, mais on ne peut exclure un passage par Panama, Lima, le lac Titicaca et les volcans de Bolivie jusqu'au Tucumán et au Paraguay. La toponymie prétendue ne révèle certes aucun lien avec celle que rapportent les relations espagnoles, mais ce qu'il décrit n'est pas invraisemblable: des enclaves incas sont signalées dans les Charcas, le Chaco et le Tucumán ${ }^{8}$. Mis en alerte par les incertitudes des manuscrits de Vincent Leblanc qui situent aussi Couran, Tabin et Seiton en Éthiopie, Bergeron a éliminé toutes les traces de ce voyage, ne conservant que le Caramel. Les récits greffent le Brésil sur ce segment, et citent ou copient Acosta, Thevet, Léry, et les capucins du Maranhão. 
Fig. 6. - Le voyage aux Indes occidentales, 1597, I'Amérique du Sud.

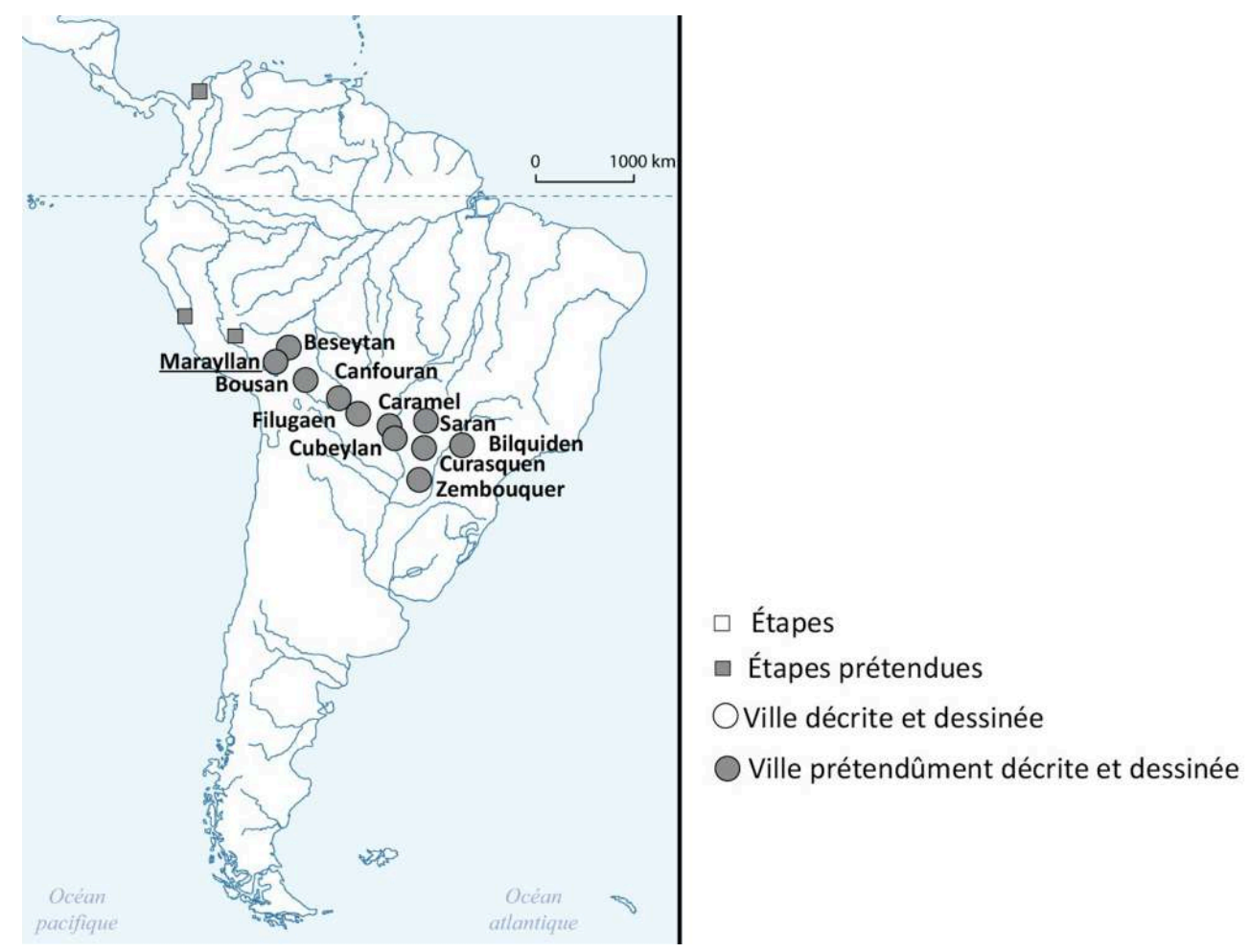

Doc. H. Bresc.

Après son retour, Vincent Leblanc s'installe à Arles et rédige ces longs mémoires, dédiant le ms. fr. 2033 à la noblesse de la vieille capitale rhodanienne.

\section{Les deux rédactions}

\section{L'auteur}

Dans les Voyages, Geoffroy Atkinson ${ }^{9}$ distinguait deux auteurs, un commerçant naïf, crédule et amateur de situations et d'accidents dramatiques, et un juriste, idéaliste et sensible à l'utopie, qui exalte la justice, la liberté religieuse et défend l'expansion de la France outre-mer. L'étude des manuscrits rétablit l'unité de la figure de Vincent Leblanc : catholique convaincu, méfiant envers les protestants « de povre silogisme » et dégoûté par la cruauté des pirates rochellois, il manifeste aussi de la sympathie pour le capitaine Ribaud, huguenot anti-esclavagiste, et pour la coexistence religieuse à Cambaye. Sa familiarité avec les jésuites des Indes se combine avec une image négative de l'Inquisition de Diu et des réquisitoires contre les Portugais aux Indes, cruels, orgueilleux et paresseux et contre la cupidité des Espagnols, mesquins, misérables, «tyrans ", que leur cruauté met « hors de l'humanité ». La dénonciation des ambitions anglaises qui effacent la toponymie française de l'Amérique du Nord semble au contraire un ajout de Bergeron.

21 Son patriotisme français est nuancé de protestation contre les princes. Il leur oppose l'exemple des seigneurs abyssins, fidélité, zèle pieux et simplicité des mœurs, dénonce les Français parricides livrés à la guerre civile et ironise sur la Française qui boit et laisse «troser son coutilhon » : elle devrait imiter la sobriété et la fidélité des femmes 
Tupi du Brésil. Ce patriotisme se conjugue avec l'affichage de ses racines italiennes et de sa condition de provençal. La Provence lui sert d'étalon de comparaison quand il évoque les particularités des pays traversés.

Sa culture polymorphe, faite de bribes, s'étend à la médecine, à la botanique, et aux savoirs exotiques, thérapeutique, astrologie et astronomie ; il manifeste sa confiance dans les connaissances scientifiques des Indiens. Il présente un intérêt obsessionnel pour la magie, la sorcellerie, l'occulte, soutenu par le sentiment profond et répété que « le diable est le prince du monde ${ }^{10}$ ».

Son désir de voyages et sa curiosité sont infinis, bien au-delà des pays visités : ses éditeurs ont supprimé une liste d'îles et de villes, depuis l'Insulinde jusqu'au Japon, Moluques, Célèbes, archipel de Saint-Lazare, Bornéo, Chapourane (Tanjampura, sud de Bornéo), îles des Larrons (Mariannes) et Lequies (Ryû Kyû).

\section{Les observations de Vincent Leblanc}

Les descriptions sont d'abord géographiques, îles, rares montagnes, campagnes plus rares encore, villes très nombreuses. Elles sont conformes aux canons du récit de voyage: les paysages sont toujours plaisants, riches, plantureux, sauf la haute montagne péruvienne. Les eaux, lacs, fleuves, mascarets, les plantes et les animaux, en particulier de l'Amérique, sont toujours remarquables. Et les notices ethnographiques couvrent un large éventail : rituels de mariage, fidélité et adultère, obsèques, en particulier le satî des veuves indiennes, alimentation (couscous, coco, caouin, farine de manioc, patate, farine de poisson, viande crue selon les pays), vêtement (les robes fendues des Pégouanes et des Siamoises), habitat, famille (femmes lascives et filles libres, dépucelage par les étrangers, mariage, fidélité), décor corporel de plumes et de labrets, musique et chansons. On note cependant l'absence de particularités connues d'autres voyageurs : le thé, le café, le piment fort ou encore l'amok, attesté en Inde.

Ces observations amènent une comparaison avec des réalités familières : maisons de Pegu et cabanes camarguaises, plaine marocaine et Camargue, Mont Gourana, au Pérou, et Notre-Dame de la Garde ou Montmartre. Les armées du Négus, nombreuses, bien vêtues, bien nourries, supportent la comparaison avec celles du roi d'Espagne, affamées et en loques. La taille des villes est ramenée à des étalons, Milan pour Calicut, Sienne et Florence pour les villes abyssines.

Les observations sur les religions sont en revanche discontinues et incertaines : Vincent Leblanc ne décrit pas les dieux des "Indiens ", qu'il réduit au Duma, c'est-à-dire à un démon, mais il rapporte des exercices de magie, de transe, le suicide religieux et la procession meurtrière du char, qu'on dira de Jaggernath, ou encore le rituel cannibale, enveloppés dans une commune réprobation. Il décrit aussi partout une prétendue vénération de la Vierge et d'un dieu à triple figure qu'il attribue à une ancienne évangélisation. Dans une tradition bien établie, il fait l'éloge de l'austérité et du pacifisme des brahmanes et des jaïns et cette appréciation des sagesses païennes recoupe une critique discrète de la France : plusieurs prosopopées s'inspirent de celle de Léry, éloge de la nudité, de la solidarité, du bon voisinage, et ironie sur la folie des voyages pour venir couper du bois au Brésil.

Les observations politiques exaltent le modèle d'une monarchie sacralisée, d'une justice à la fois rigoureuse et humaine : théocratie royale, sacralité et armées nombreuses en Pégou, justice et paix, sécurité sociale, camp impérial, princes emprisonnés en Éthiopie. 
Non sans quelques blâmes : il réprouve au Monomotapa le rôle des épouses royales dans l'élection du successeur et à Pidir la succession des rois par l'assassinat.

\section{La méthode de Vincent Leblanc} pas personnelles: Vincent Leblanc donne des garants, un peintre portugais qui a visité la Tartarie, Guilhem de Cassis pour Ceylan, le sieur de La Courbe pour São Tomé ; un truchement arabe, Halis, et un ambassadeur espagnol rencontré en Éthiopie l'ont informé sur la Nubie, Bernardin Nadal sur le régime ottoman, etc. Et il cite les livres qu'il a lus, Cosmographie de Belleforest, voyages de Martin de Vitré et de Pyrard de Laval, Historia Gothorum de Jean Magnus, Histoire de Portugal de Jeronimo Osorio. S'agit-il de plagiat? La prudence s'impose: les emprunts peuvent être une confirmation de l'observation personnelle, une garantie. Les voyages les plus novateurs englobent des circuits fictifs et l'appropriation des savoirs est chez tous une forme d'innutrition déguisée sous l'autopsie déclarée. Mais Vincent Leblanc emprunte des pans entiers de récit et plusieurs dessins sans le signaler.

M. Laugaa a mis en lumière le désir de Vincent Leblanc de composer un livre en unissant des documentations avérées par son expérience du terrain et de faire œuvre théorique dans le champ de la cosmographie ${ }^{11}$. Les manuscrits ne présentent certes aucun ordre, ni chronologique ni thématique. L'entrelacement et la confusion des dates conduisent à des contradictions et à des contaminations. En mai 1631, chez Peiresc, Vincent Leblanc reconnaît avoir placé en Amérique des régions d'Afrique. Mais les manuscrits sont prêts pour l'édition, garnis de titres de chapitres, de poèmes introductifs, d'intitulés alléchants pour les anecdotes (Discours récréatif, discours remarquable, conte fasésieus). L'effort littéraire s'exprime d'abord par l'accumulation des anecdotes: Mariela Witek ${ }^{12}$ a analysé les effets de réel, d'authenticité, les scènes pathétiques et fascinantes, dans les anecdotes conservées par Bergeron, et mis en lumière la volonté de divertir et de garder l'attention, et les efforts d'édification morale; violence, souffrance, mort scandent la destinée pitoyable d'un personnage balloté par le destin, suscitant pitié et sympathie, comme dans d'autres récits de voyage. Ces anecdotes font partie du dessein propre de Vincent Leblanc, écrivain mal armé, mais habile: les manuscrits comptent plus de cent cinquante anecdotes qui recyclent des thèmes folkloriques et littéraires, rapportent des miracles, des récits historiques, des épisodes de sortilèges et d'interventions démoniaques, des récits érotiques ou pornographiques, de brutales anecdotes scatologiques, de dures plaisanteries, les « cassades ». Guilhem de Cassis y joue constamment le rôle du clown, escroc et voleur, obsédé de femmes, partout trompé, battu, moqué. Les manuscrits sont scandés de clés de véracité :

"J'ai veu », " nous étions en Perse ", « nous passâmes... ", " nous partîmes avec une troupe de marchands. »

Vincent Leblanc invoque son carnet de notes :

«Mon petit livret ou mémorial où je mettais plusieurs curiosités... sur l'assiette du pays, qualité, rois et gouvernement ${ }^{13}$."

Et les effets de réel sont innombrables dans les récits les moins vraisemblables, pirogue monoxyle en Caramel, raquettes au Canada, églises taillées dans le roc le long du Nil, « peuple embastonné avec chiens » dans le désert, rhinocéros en Nubie. 


\section{Les apports de Bergeron et de Coulon} emprunts créent un espace entre le vrai et le faux qui fédère les mondes visités et décrits. Dans les manuscrits, les «signifiants iconiques » omis par Bergeron et Coulon participent à la création de cet entre-deux, gages de sincérité (croquis d'Aden, de Goa ou d'un paysage de la montagne éthiopienne) empruntés peut-être à d'autres voyageurs et images fausses et mensongères (images de Janibarou, ville de Madagascar, ou de Caramel sur le fleuve Paraguay). Toutes collaborent à unifier Amériques, Éthiopie et Indes orientales: même religion démoniaque, mêmes prêtres sacrificateurs, les chaoury, et, d'une région à l'autre. Vincent Leblanc multiplie les transferts, les mots incas tanbo (auberge) et mamacouna (vierge consacrée) au Pégou, le satî en Amérique, le jeu de ballon mexicain à Java. Il unifie l'habillement : les femmes de Nouvelle Espagne portent la robe sans couture (le sari) tandis que les hommes se contentent d'un étui pénien. Les contaminations sont quelquefois étonnantes : ainsi, la langue des doctes de Caramel est le mandarin, « leur latin ».

\section{Allotopies et pays de synthèse}

Comme les auteurs d'utopies, Vincent Leblanc choisit pour ses pays virtuels des terres lointaines. Ce sont d'abord des allotopies, synthèses d'observations collectées (fig. 7). Le Pégou réunit ainsi les traits communs à toute l'Inde, hindouisme shivaite, satî, rituels cruels, et ceux d'une société bien gouvernée : le roi y est divin, incontesté, antithèse de 
la France d'esprit « libertin »; il distribue ses rentes, dispose d'une armée considérable, assure une justice rigoureuse. Tout ici n'est pas imaginaire : la divinité du monarque, la présence de l'hindouisme souterrain et des rites ancestraux ont imprégné les royaumes hindouisés. La Transiane voisine présente une figure plaisante : une belle province, des chevaux, des jardins, des femmes avenantes et courtoises au vêtement échancré, des filles libres (les pères sont obligés de nourrir les enfants de leurs amours extranuptiales), des fêtes, des danses, de la musique, mais aussi une loi qui oblige les mères à allaiter leurs enfants et un service militaire universel. Ce serait un paradis terrestre sans les sacrifices offerts au Duma.

Fig. 7. - Les pays virtuels de Vincent Leblanc.

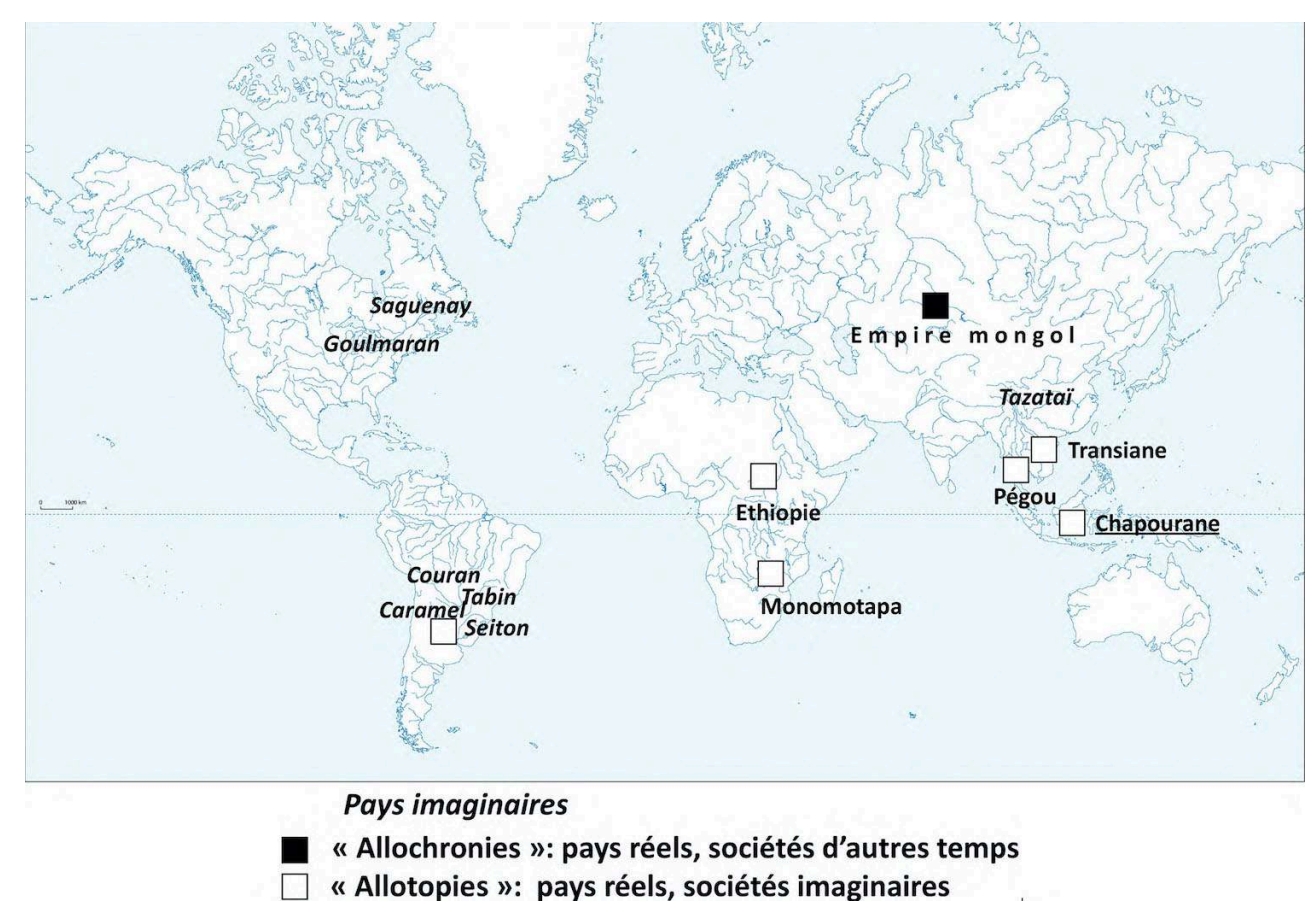

Doc. H. Bresc.

Chapourane (Tanjampura à Bornéo) est un « jardin du monde », riche de fruits, de clous de girofle, de cannelle, de bois odoriférants, de myrobalan, qui garantit la longévité, et de perles. Cet autre paradis terrestre est assombri par les sacrifices humains et l'absence de règles interdisant l'endogamie. La même antinomie marque le Mongibir: amitié universelle, accueil aux étrangers, mariage sans querelle, honnêteté, prospérité contrastent avec le paganisme. Au Couran, bon gouvernement, fraternité, honnêteté, chasteté, dévotion, sécurité sociale (aveugles entretenus par le Trésor public, travail assuré aux voyageurs, pauvreté éliminée), contrastent avec le culte de l'idole Apolita. Le Seiton voisin est plus proche de l'Éthiopie idéale : honnêteté des transactions, absence de jurement, de repas solitaire, "set royaume rampli de tout bien et privé de visse " connaît aussi la dévotion à la Vierge; de même le Carfouran où abondent légumes et fruits, où la vie est pacifique et l'amitié réciproque, sans conflits entre époux et sans séparations, sans jurement, et animé par la haine du mensonge et du vice.

L'Éthiopie, enfin, est un modèle d'allotopie chrétienne: gouvernement juste, lois affichées et portées à la connaissance de tous, punition de l'adultère, du vagabondage et de la prostitution, élimination de la pauvreté et pratique de la redistribution, 
souverain pieux et courageux, peuple vertueux et fidèle à ses rois, antithèse de la France, "un seul Dieu, [...] une foy et une loy», et les royaumes voisins, Mélinde, Iscande, Soba, en sont le reflet.

Une véritable "allochronie » apparaît enfin, construite sur les lectures : la Mongolie unit à l'empire récemment restauré par Altan Khan (1542-1582) la mémoire de celui des Gengiskhanides, de la mer de Mangi (Chine) jusqu'à la Caspienne.

\section{Les utopies primitives}

40 Très conscient de la férocité du rituel cannibale, Vincent Leblanc n'adhère pas au primitivisme, à l'âge d'or décrit par Pierre Martyr d'Anghiera, pays sans lois, sans code, sans juge, peuples sans F ni R ni L (ni Foi, ni Roi, ni Loi) ${ }^{15}$, mais, loin de refouler le sauvage dans la déraison, il subordonne les peuples nus à une monarchie de type incaïque. En Caramel, un prince, une cour avec ses officiers et ses «Jours » de justice, un palais, une noblesse vêtue encadrent ainsi un peuple tupi-guarani nu; le culte et les sacrifices cannibales, le communisme des biens, et la guerre nue au corps à corps destinée à faire des prisonniers, les sacrifier et les boucaner, y contrastent avec des savoirs remarquables, une architecture incaïque et une artillerie fort ancienne.

41 La valorisation du primitif, presque du "bon sauvage", se rencontre dans la description des Goulmaran, situés au Canada : vie simple, en cavernes, robustesse des corps, égalité et fraternité, communion des biens. Leur croyance en l'immortalité de l'âme fonde une précoce religion naturelle.

\section{L'héritage du monde de Vincent Leblanc}

La méfiance a été immédiate chez les érudits comme chez les voyageurs qui repèrent emprunts et invraisemblances, mais l'ampleur du consentement est stupéfiante : La Martinière, en 1726, sait que Vincent Leblanc " crée des villes et des royaumes ${ }^{16}$ », mais il intègre Madrogan. En 1660, D’Avity incorpore des bribes de la Madagascar de Vincent Leblanc et la ville de Delbequi en Moyenne-Égypte. Jusqu'au début du XIXe siècle, les dictionnaires citent les villes et les provinces que Vincent Leblanc prétend avoir visitées, en Afrique orientale et en Asie du Sud-Est, Albiar, Canarana, Cassubi, le Goulmaran, le Tazatay, la Transiane, Mandranela, le Sinabo, Magourres, Patanes. Belugara est encore présent dans le dictionnaire de Trévoux en 1771; Seiton et Madrogan ont l'honneur de figurer dans le dictionnaire de l'Encyclopédie en 1785 (fig. 8). Seule l'exploration en viendra à bout, et ce non sans peine, comme le montre l'analyse précise de William Randles ${ }^{17}$ : la description du palais de Monomotapa est reproduite en 1775 par Dubois Fontenelles, et en 1788 par Millar, alors que le missionnaire Mauriz Thoman a révélé dès 1759 que ce n'était qu'un « kraal » de roseaux et de pieux. Les Sources de l'histoire de l'Afrique au Sud du Sahara, énumèrent encore en 1971 les villes imaginaires de Vincent Leblanc. L'internet permet de débusquer ces emprunts et de constater qu'aucune barrière de sécurité n'est posée à l'utilisation de ce « savoir » biaisé et toxique. 
Fig. 8. - L'héritage de la géographie de Vincent Leblanc.

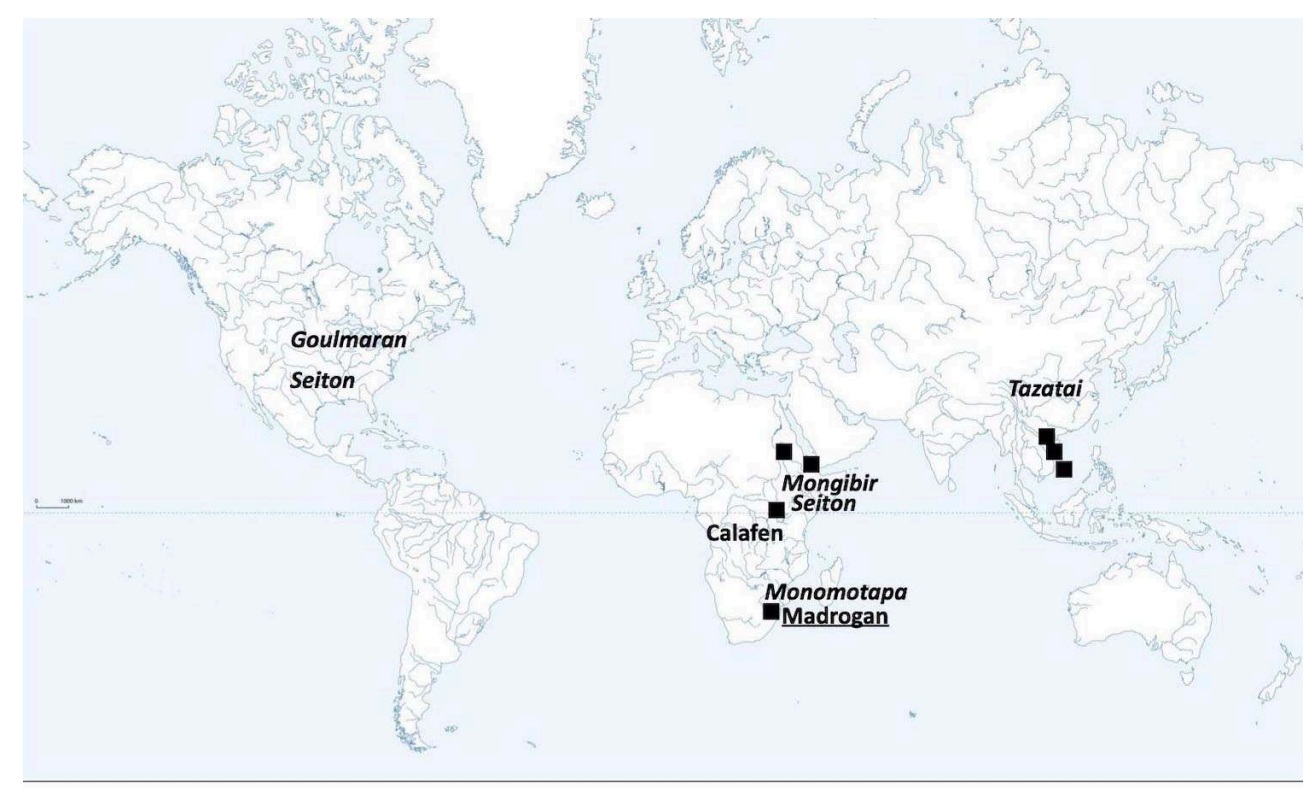
Pays imaginaires
- Villes imaginaires
$\square$ « Allotopies »: pays réels, sociétés imaginaires

Doc. H. Bresc.

La postérité de Vincent Leblanc est encore plus touffue dans l'histoire littéraire du XVIII ${ }^{\mathrm{e}}$ au $\mathrm{Xx}^{\mathrm{e}}$ siècle : les voyages extraordinaires et les anticipations géographiques, souvent combinées avec le roman picaresque, s'épanouissent, de Candide à Jules Verne et Julien Gracq. Et si on soupçonne des emprunts, ici et là, aux Voyages, l'essentiel est dans la transmission ou la réinvention de la méthode, de l'inventivité et des combinaisons audacieuses de notre voyageur.

\section{BIBLIOGRAPHIE}

ATKINSON Geoffroy, 1920, The Extraordinary Voyage in the French Literature before 1700, New-York, Columbia University Press.

BECKMANN Johann, 1807-1810, Litteratur der älteren Reisebeschreibungen, Göttingen, (2 vol.).

(LE)BLANC Vincent, 1634, Histoire geografique et memorable de l'assiete de la terre universelle, Aix, Estienne David.

GASSENDI Pierre, 1992, Vie de l'illustre Nicolas-Claude Peiresc, conseiller au Parlement d'Aix, trad. Roger Lassalle, Paris, Belin.

HOLTZ Grégoire, 2011, L'Ombre de l'auteur. Pierre Bergeron et l'écriture du voyage à la fin de la Renaissance, Genève, Droz. 
LAUGAA Maurice, 1993, « Les Voyages fameux du sieur Vincent Le blanc », dans Rizza C. (dir.), La Découverte de nouveaux mondes. Aventures et voyages imaginaires au XVII siècle, Fasano, Schena.

LE BLANC Vincent, 1648, Les voyages fameux du sieur Vincent Leblanc marseillais, Paris, Gervais Clousier, disponible en ligne.

LEVILLIER Roberto, 1927, Nueva Crónica de la Conquista del Tucumán, Madrid.

LINDSAY Robert O., 1979, « Les Voyages Fameux : a seventeenth-century Travel Account ", Australian Journal of French Studies, XVI, p. 323-338.

MAROUBY Christian, 1990, Utopie et primitivisme. Essai sur l'imaginaire anthropologique à l'âge classique, Paris, Seuil.

RANDLES William G. L., 1959, L'image du Sud-Est africain dans la littérature européenne au XVI siècle, Lisbonne, Centro de Estudos Históricos Ultramarinos.

WITEK Mirella, 2018, « Les Anecdotes dans les récits de voyage du XVII ${ }^{\mathrm{e}}$ siècle », thèse de philosophie, Toronto, disponible en ligne.

\section{NOTES}

1. Gassendi 1992, p. 147-148.

2. Holtz 2011, p. 350 sq.

3. Les mss. fr. 5652 (306 fol., Segon livre de la monarchie articane et antarticane) ; fr. 5590 (455 fol., Livre de l'histoire géographique et description de toute la terre comprize en une juste émisfère) ; fr. 5591 (274 fol., Les Voyages é péregrinasions de Vincens Blanc marseilhès); fr. 5592 (170 fol., De la grande monarchie entarticane, tratant des quriosités des Indes oriantalles et oxidentale) ; fr. 2033 (164 fol., Histoire naturelle de l'Indie osidentale).

4. Laugaa 1993, p. 257.

5. Holtz 2011, p. 337.

6. Atkinson 1920, p. 27.

7. Leblanc 1640, II, p. 149.

8. Levillier 1927, p. 27.

9. Atkinson 1920, p. 28.

10. (Le)Blanc 1634, p. 4.

11. Laugaa 1993, p. 272.

12. Witek 2018.

13. BnF, ms.fr. 5591, f. $87 \mathrm{v}$.

14. Lindsay 1979, p. 337

15. Marouby 1990, p. 193.

16. Beckmann 1808, I, 3, p. 554.

17. Randles 1959, p. 96-98. 


\section{RÉSUMÉS}

Marchand, marin, mercenaire, Vincent Leblanc a quitté Marseille en 1567 pour soixante ans de voyages dans les quatre parties du monde. À son retour, il rédige de longs mémoires qu'il fait parvenir, non sans réticences, à Peiresc. Ce dernier en confie l'édition à Pierre Bergeron, qui les remanie, les enrichit de références, supprime les élucubrations de Vincent Leblanc niant la rotondité du monde et en fait un best-seller publié en 1640. Les mémoires autographes, conservés à la Bibliothèque nationale, sont eux-mêmes un feuilleté d'observations, de réminiscences livresques, de plagiats, un roman picaresque aussi, et construisent des pays utopiques aux marges du monde réel, «allotopies» comme le Pégou (Birmanie), résumé du monde indien, ou le Caramel (Paraguay), synthèse entre Brésil des Tupinambas et société inca. Ces royaumes et ces villes chimériques que l'éditeur n'a pas éliminés, parcelles d'un monde virtuel, passent dans le savoir géographique jusqu'au XIX ${ }^{e}$ siècle.

\section{AUTEUR}

\section{HENRI BRESC}

Professeur retraité, Université de Paris X-Nanterre 


\title{
Le trésor perdu de l'atoll de Pinaki (archipel des Tuamotu, Polynésie française) vers 1859-1994 au moins...
}

\author{
Véronique Dorbe-Larcade
}

1 Dans le ressort de ce qui constitue à présent la Polynésie française, la recherche, entre le début et la fin $d u x^{e}$ siècle, du butin caché par des pirates dans l'une des îles Tuamotu, vers le milieu du xIx siècle, relève assez exactement de la définition du fait divers et ce terme, issu du lexique du journalisme, peut lui être parfaitement appliqué. Il renvoie, sémantiquement, à la logique et aux outils de la communication, selon deux dimensions privilégiées: celle du sensationnel (qui suscite l'émotion) et celle du retentissement (qui suscite la mobilisation et l'intervention des autorités, notamment forces de l'ordre et appareil judiciaire). L'entreprise ne saurait pourtant être réduite à ce seul cadre d'interprétation.

Plusieurs « chasses au trésor » autour de l'atoll de Pinaki sont donc documentées - sans être exactement attestées - en 1913-1914, puis en 1921 (pour juin et juillet), encore en 1934, enfin en 1993-1994. Il existe bel et bien des sources officielles ainsi que des articles de presse qui authentifient la réalisation d'expéditions vers cette destination. Par ailleurs, des traces subsistent de la présence et des agissements à Tahiti des promoteurs de ces chasses au trésor, ainsi dans les mouvements de navires enregistrés au port de Papeete. Les mémoires et recueils de souvenirs de témoins de la vie tahitienne à la fin $\mathrm{xIx}^{\mathrm{e}}$ et au début $\mathrm{xx}^{\mathrm{e}}$ siècle mentionnent par ailleurs les aventuriers et personnages louches (sinon impliqués dans des affaires criminelles) qui s'y trouvent mêlés. Cependant, le doute plane sur la véracité de ce qui en cause; en raison du caractère invérifiable - quoique plausible - des informations concernant l'origine et les conditions d'enfouissement du trésor ainsi que sur la localisation et l'issue des recherches et des fouilles à Pinaki ou à proximité.

A priori, on a affaire à une " histoire " qui ne relève guère de l'Histoire. Sa récurrence et sa longévité interpellent cependant et incitent à s'interroger sur ce qu'est et ce qui fait la substance de cette «fable». C'est le propos de ce qui suit. Il s'agit, dans un premier temps, d'inventorier les ressorts de l'intrigue qui s'est nouée : son origine, ses 
péripéties, et de mettre en lumière le rôle de ses protagonistes; afin, dans un second temps, de démonter la mécanique des procédés et des circonstances qui la rendent possible et efficace.

\section{Une intrigue}

4 L'affaire du trésor de Pinaki relève d'une accumulation d'histoires emboîtées. Il faut les rapporter au conditionnel dans la mesure où elles reposent sur le témoignage d'un seul personnage qui se réfère à des faits et à des personnes dont l'existence même est incertaine. Au point de départ, vers le milieu du XIXe siècle, alors que le désordre faisait rage à l'occasion de la guerre civile de 1857-1859, quatre mercenaires auraient volé pas moins de quatorze tonnes d'or dans une église de Pisco au Pérou ${ }^{1}$. L'ampleur de ce butin, les conditions de la fuite à laquelle les voleurs se trouvèrent contraints, les auraient obligés à abandonner la plus grande partie du trésor en l'enterrant dans un atoll corallien minuscule du Pacifique, l'un de ceux de l'archipel des Tuamotu. Celui-ci était doté de caractéristiques topographiques susceptibles de permettre de le retrouver, et les pillards retinrent pour le désigner le nom de Pinaki. Les quatre hommes se seraient ensuite rendus en Australie. Deux d'entre eux y auraient trouvé la mort, tués par des Aborigènes, tandis que les deux autres auraient été condamnés à une lourde peine de prison pour meurtre. Juste avant sa mort le mercenaire survivant, Killorain, aurait raconté l'histoire au prospecteur Charles Edward Howe, le premier protagoniste de l'affaire qui soit bien identifié . Sans être complètement invraisemblable, l'odyssée des pillards n'est pas formellement prouvée. Ainsi, alors que les quatre voleurs du trésor de Pisco seraient arrivés à Papeete en décembre 1850, sur le schooner Bos'n Bird, le catalogue des mouvements de navires dans ce port indique seulement en 1849 : entrée le 22 novembre - sortie le 16 décembre d'un navire non identifié (unidentified [ship]) sans indication sur son port de provenance ${ }^{3}$.

5 En tout état de cause, au début du $\mathrm{xx}^{\mathrm{e}}$ siècle, Howe, établi à Tahiti, fut à l'origine d'opérations de recherche qui s'étalèrent sur 13 ans, et qui connurent trois épisodes marquants. Le premier épisode intervint fin 1913: quelques notables de la société tahitienne de l'époque, Franck Homes, Alexandre Le Gayic et Georges Spitz ${ }^{4}$, passèrent contrat avec lui pour financer l'équipement d'un navire et d'une campagne de fouilles ainsi que pour partager les gains escomptés ${ }^{5}$. Dès l'accord passé, la goélette la Suzanne largua ses amarres avec, à son bord, trois de ces associés : le capitaine Le Gayic, Spitz et Howe. En résulta la mobilisation de gendarmes pour occuper l'îlot de Pinaki et contrôler les travaux de fouille. Cette mission de gendarmerie fut sans résultat tout comme l'expédition conduite par Le Gayic, semble-t-il ${ }^{6}$.

6 Une deuxième campagne de fouilles attestée eut lieu en 1921. Alors, Charles Edward Howe qui, gagne-petit à la limite de la mendicité ${ }^{7}$, travaillait dans un magasin de souvenirs à Papeete rencontra les frères Juventin. Ils étaient six : Louis, Auguste, Élie, Émile, Édouard et Henri, issus d'une famille de négociants-entrepreneurs ayant pignon sur rue ${ }^{8}$. Il parvint à les convaincre de l'aider, à leur tour, à mettre à jour le fameux trésor. Et au mois de juin 1921, ayant armé un vieux cotre, la Florina, ils s'embarquèrent pour Pinaki. Sur place, il s'avéra que la « piscine » qu'ils s'étaient mis à fouiller était en fait une fosse très profonde et que leur équipement était complètement inadapté. Chaque fois qu'ils creusaient au fond de l'eau, ils soulevaient des nuages de sable, ce qui les contraignait à remonter à la surface. Il devenait donc évident pour eux, munis 
seulement de pelles et de lunettes de plongée, qu'il serait quasiment impossible de retrouver le trésor. Aussi abandonnèrent-ils, et mirent le cap sur Papeete ${ }^{9}$. Une troisième expédition vers Pinaki fut montée en juillet 1921. Charles Edward Howe et les frères Juventin y participèrent, tandis que Chin $\mathrm{Foo}^{10}$, un homme d'affaires et banquier d'origine chinoise, établi à Papeete, en assura le financement. Cette fois-ci, Howe vint avec eux ainsi qu'un charpentier, Clinton Chapman. Quoiqu'un important matériel ait été embarqué sur la goélette Vaihiria, l'opération ne fut pas concluante. Au bout de trois jours, complètement découragés et malgré les trésors d'ingéniosité déployés par Chapman pour trouver un système qui empêcherait le trou de se combler de sable, les recherches furent abandonnées ${ }^{11}$.

7 La chasse au trésor de Pinaki n'en continua pas moins. De longue date, Howe prétendait avoir retrouvé le magot ${ }^{12}$. Il aurait effectivement déterré une partie au moins du trésor, mais dans un autre atoll que celui de Pinaki (en raison d'une erreur de toponymie de la part des mercenaires qui avaient enfoui le trésor initialement), mais il aurait été contraint, faute de pouvoir le transporter, de l'enterrer à nouveau. Son attitude déconcertante et fuyante, au retour à Papeete de la première " expédition Juventin ", pouvait donner à penser qu'il en savait plus qu'il n'en disait ${ }^{13}$. La chose était d'autant plus aisément envisageable qu'Howe avait pris femme à Pinaki, ce qui lui donnait accès à la terre, facilitant grandement les fouilles, pour lui, tout en permettant de fréquentes allées et venues sans attirer l'attention ${ }^{14}$. Sa disparition en Australie, après son expulsion de Tahiti en 1928, alors qu'il rassemblait des fonds et engageait une équipe pour une nouvelle expédition vers Pinaki ${ }^{15}$ acheva de laisser planer le doute.

8 C'est alors, à la fin des années 1930, que se manifesta un protagoniste décisif de cette chasse au trésor en la personne de George Hamilton. S'il en vint à jouer un rôle-clé, c'est moins sur le terrain que dans les imaginations, car il contribua pour beaucoup à la construction, à la narration et à la notoriété de l'affaire, par la publication, en anglais, à Londres, en 1939 du livre The Treasure of The Tuamotus. Cette publication se présentait comme des mémoires d'aventure vécue. L'auteur visait assez clairement un succès d'édition, générateur de droits d'auteur substantiels, alors que, de son propre aveu, il se trouvait en situation financière précaire. Il comptait aussi de la sorte donner du crédit à la poursuite du "trésor de Pinaki ", dans la mesure, en reconstituant et en attestant (sans fournir de preuves vérifiables) de la véracité l'histoire de Killorain et de celle de Howe. En fait, ne s'agissait-il pas tout simplement d'une mystification? Il rapporte ainsi que, spécialiste de plongée et résidant en Angleterre, il aurait été attiré par une petite annonce de presse (dont il n'est pas possible de trouver trace) en Australie dans la quête de Howe, reprise par un certain Edwardes, prospecteur. C'est en sa compagnie qu'il se serait rendu sur l'atoll de Pinaki en janvier 1934. N'y trouvant pas les lieux décrits par le forban qui avait prétendument enfoui le premier le butin, et confortés par les dires de familiers de cette zone des Tuamotu, ils auraient parcouru plusieurs atolls, et se seraient enfin trouvés devant une "piscine [corallienne] en forme de poire", conforme aux descriptions de Killorain. Ils se heurtèrent là aux mêmes problèmes d'excavation que ceux rencontrés par les Juventin. Hamilton y ajoute la relation détaillée - palpitante, mais quelque peu hors de propos - de deux combats spectaculaires, alors qu'il était en plongée dans ce bassin, d'abord contre une pieuvre géante, puis, une autre fois, contre une énorme murène qu'il finit par tuer. Mais il se garde, dans son livre, de localiser et même de nommer l'atoll sur lequel il a effectué ses fouilles. Il insiste par contre sur le fait que, faute de matériel pour aspirer le sable et dégager les lingots, lui et son associé étaient retournés à Papeete chercher des fonds 
pour une nouvelle expédition, mais que leurs bailleurs avaient refusé d'apurer les pertes et de réinvestir dans l'aventure qui se termina donc. On perd la trace de George Hamilton, probablement emporté dans la tourmente de la Seconde Guerre mondiale, il a eu cependant une postérité. En 1993-1994, en effet, une nouvelle campagne de fouilles eut lieu, diligentée par un personnage présenté comme un descendant de l'auteur du Treasure of The Tuamotus. Partant de Fakarava, le navire affrété à ce dessein mit cette fois le cap sur l'atoll de Tepoto. Car, d'après une photo ancienne, il correspondrait au site véritable du trésor. Mais, peu après l'arrivée sur cette île, le temps se gâta au point que les membres de l'expédition échappèrent de peu à la mort sur le récif, tandis que la chasse au trésor tournait court ${ }^{16}$. En tout état de cause, des traces de fouilles anciennes sont aujourd'hui visibles en une douzaine d'endroits à Pinaki, pour la plupart aux environs de la seule passe de l'atoll qui est trop étroite, même pour que des canots pneumatiques puissent l'emprunter, comme le montre le reportage documentaire réalisé récemment par l'une des chaînes de télévision de Polynésie française ${ }^{17}$.

\section{Dénouement et interprétation}

Paradoxalement, l'absence de résultats concrets et probants de ces expéditions successives, au lieu de faire perdre toute crédibilité au «trésor de Pinaki» semble plutôt avoir corroboré sa véridicité. Dès lors, il conviendrait de lui appliquer l'équivalent "insulaire» de la notion de "légende urbaine», désormais théorisée ${ }^{18}$. Distincte du canular et de la rumeur, elle s'apparente plutôt au mythe, dans une version contemporaine. La nature légendaire de ce qui se rapporte à Pinaki est illustrée, par exemple, par le propos répété que, la nuit notamment, le trésor serait gardé par les esprits de deux Blancs et d'un Noir (tués, les uns et les autres, pour éliminer les témoins, après que l'or ait été enterré19).

10 Un tel phénomène s'affirme d'autant plus qu'existe une certaine tradition de "fiction " à propos de la Polynésie. Celle-ci revêt deux dimensions : d'une part, on la présente comme un envers utopique de l'Europe. On pense évidemment à l'idéalisation de Tahiti dans les écrits des voyageurs européens du XvIII ${ }^{e}$ siècle en un "nouvel Eden » idyllique, harmonieux et heureux, dont les habitants, faute d'être exactement de «bons sauvages ", connaissent une société et des mœurs plus conformes à la Nature de l'humanité. Les écrits de Bougainville et de Diderot en sont l'expression la plus fameuse ${ }^{20}$. Corollairement, dans l'imaginaire, cette partie de la planète est volontiers conçue comme un lieu de subversion et de liberté absolues. En tout état de cause, l'océan Pacifique est un espace d'activité pour les pirates. Entre autres, William Dampier (1651-1715) dont le récit des expéditions en 1683-1691 puis 1699 et enfin en 1701 et 1706 où figurait l'histoire d'Alexandre Selkirk : à l'origine de l'histoire de Robinson Crusoé fut un grand succès en librairie et marqua les esprits ${ }^{21}$.

11 Surtout, Tahiti et les îles circonvoisines sont le théâtre de l'aventure des mutins de la Bounty, menés par Fletcher Christian (événements survenus en 1789-90). Elle fut popularisée tout particulièrement, en 1932, par James Norman Hall et Charles Nordhoff dans la trilogie romanesque : Les révoltés du Bounty ; Dix-neuf hommes à la mer ; Pitcairn ${ }^{22}$.

Un autre facteur déterminant de la longévité de la «légende» de Pinaki et de la répétition des chasses au trésor relève certainement de la situation coloniale dans laquelle se trouvaient l'archipel de la Société et celui des Tuamotu jusque dans la seconde moitié $\mathrm{du} \mathrm{xx}^{\mathrm{e}}$ siècle, d'abord dans le cadre d'un régime de protectorat à partir 
de 1843, puis de colonie au sein des Établissements Français d'Océanie (E.F.O.) après $1880^{23}$. Tout aussi fortement a pu jouer la situation d'enclavement qui caractérise alors la région. À la fin du $\mathrm{xIX}^{\mathrm{e}}$ siècle, il fallait plus de soixante-dix jours pour toucher Papeete au départ de Marseille ou du Havre. Tahiti et les îles alentour étaient la seule colonie à ne pas être reliée directement à la métropole. Certes, à partir des années 1910, la Compagnie navale de l'Océanie envoya des vapeurs, mais toujours de façon épisodique. C'est seulement l'ouverture du canal de Panama à partir de 1914 qui changea la donne ${ }^{24}$.

Alors que le Pacifique, dans son ensemble, était largement soumis à l'influence anglophone et à l'hégémonie britannique ${ }^{25}$, la Polynésie (associée - artificiellement par l'administration française à la Nouvelle-Calédonie pour former les E.F.O) constituait, par ailleurs, une sorte d'isolat où les métropolitains comme les cadres administratifs venus de France étaient peu nombreux, sans moyens et plan d'action d'ampleur ${ }^{26}$. Le niveau de vie d'ensemble et des métropolitains d'origine y est relativement $\operatorname{bas}^{27}$ : la perspective de trouver un trésor et de faire ainsi fortune rapidement peut-être d'autant plus séduisante ${ }^{28}$. De plus, manifeste dès le début de la période coloniale et illustré par le succès de Jacques-Antoine Moerenhout ${ }^{29}$, le dynamisme de négociants entreprenants s'imposa encore davantage par la suite, face à une administration défaillante, peu considérée voire méprisée, ce qui incitait aux initiatives individuelles à la limite voire au-delà de la légalité30. Il y a une certaine logique, dans cette perspective, à relever la présence lors de l'expédition montée par Howe, d'un certain nombre de figures du petit monde du commerce et des affaires de Tahiti : Le Gayic, Juventin, Home...

Enfin, l'inscription dans la durée de l'affaire du trésor de Pinaki tient certainement à ce qu'elle présente quelques-uns des principaux traits d'une "mise en récit» (ou storytelling) réussie, tels qu'ils sont théorisés et utilisés à présent en matière de promotion commerciale («marketing») et de propagande électorale ou d'outil politique. Il s'agit, contrairement à une argumentation logique classique (fondée sur la mise en évidence rapide d'un problème et amenant à l'adoption d'une solution), de capter l'attention du public visé par une " histoire » faisant appel à ses émotions voire à ses passions et de faire en sorte qu'il se l'approprie et en devienne bel et bien acteur, en allant de lui-même, pour ainsi dire, à la solution (à savoir au produit ou l'acte) apportant solution ou remède et en intégrant le problème afférent ${ }^{31}$. Assez sûrement, cette affaire mêle des menées délictueuses relevant de l'escroquerie et une sorte de délire fantasmatique collectif appelant une analyse psychologique, voire psychiatrique. Effectivement, en tout cas, il y a conjonction entre les entreprises de Howe et de Hamilton et des phénomènes culturels de masse conditionnant un imaginaire susceptible d'entraîner, susciter, déclencher un "passage à l'acte»: à savoir l'organisation concrète d'une chasse au trésor. D'abord ont pu compter la diffusion et la notoriété des écrits du célèbre naturaliste Charles Darwin (1809-1882) qui, à plusieurs reprises, pour montrer à quoi ressemblait un atoll, a utilisé la gravure de l'̂̂le de Pentecôte (Whitsunday Island), autrement dit Pinaki dans l'archipel des Tuamotu, réalisée par Frederick William Beechey (1796-1856) ${ }^{32}$ (fig. 1). 
Fig. 1. - "Whitsunday Island, Society Islands (plus exactement archipel des Tuamotu)", vers 1825. Aquarelle : 11.8 x $31.8 \mathrm{~cm}$ in box 46.8 x $34.5 \mathrm{~cm}$, par Frederick William Beechey, 1796-1856.

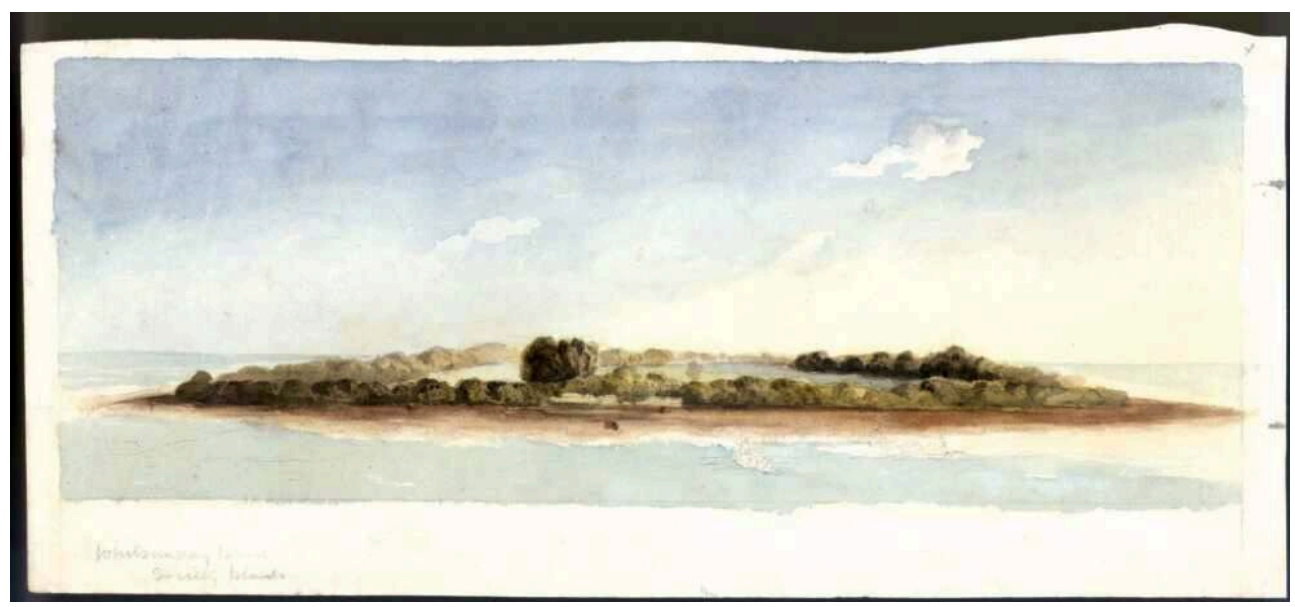

Original drawings, Pitcairn Islands, Hawaii and Pacific, Captain William Beechey, H.M. S. Blossom, 1825-28, I.I.-Rex Nan Kivell Collection NK9603/8.

Il faut constater également que les menées de Howe se placent dans le sillage de la publication de lîle au trésor de Robert-Louis Stevenson (parue entre 1881 et 1883, édition en français en 1885): grand succès littéraire de la fin du xix siècle, dont la première adaptation cinématographique, le film muet lîle au trésor de Maurice Tourneur (sorti en 1920) popularisa encore davantage l'histoire ${ }^{33}$. De même, on peut rapprocher la chasse au trésor de Hamilton de la sortie et du succès, en 1931, du film de F.-W. Murnau, Tabu qui mettait en scène la vie et les amours tragiques d'un jeune pêcheur de perles des Tuamotu ${ }^{34}$. La sortie et l'énorme succès en 1934 de la plus célèbre adaptation cinématographique de lîle au trésor: le film de Victor Fleming, avec Lionel Barrymore dans le rôle de Long John Silver, rendait les esprits encore plus propres à donner créance au butin pirate caché à Pinaki ${ }^{35}$.

Il convient particulièrement de relever que «l'histoire » du trésor de Pinaki réunit quelques-uns des éléments habituels d'une manœuvre de manipulation. Il en va ainsi $\mathrm{du}$ caractère faramineux de la valeur et du contenu du butin que l'on fait miroiter (description donnée par Killorain et rapportée par Howe selon G. Hamilton) : quatorze tonnes de lingots d'or, sept chandeliers en or ornementés de bijoux, trente-huit rivières de diamants, une quantité de bracelets, une quantité de crucifix, un coffre de pierres non taillées (probablement des rubis), un coffre de doublons espagnols. En valeur actuelle, les lingots d'or représentent environ $210000000 £$ ou environ 410000000 USD, soit un peu plus de $367000000 €$. S'y ajoute le recours à des moyens de mise en confiance assez systématiquement utilisés en cas de mise sous emprise ou influence. Il s'agit notamment de l'établissement d'une connivence, par le biais d'un "secret" transmis à des intimes. Celle-ci est, de plus, fortement liée à des circonstances émotionnelles fortes dans lesquelles la projection empathique est aisée : tout spécialement s'il est question, comme prêté à Killorain, des dernières paroles d'un mourant ou que l'on se trouve sous la pression de l'urgence, ce que décrit Hamilton ${ }^{36}$. 
17 L'affaire de Pinaki est d'autant plus susceptible de contribuer à la réflexion sur le réel et le virtuel - le thème du $144^{\mathrm{e}}$ Congrès du CTHS - que la chasse au trésor qui en a résulté a donné lieu elle-même à deux transpositions littéraires, sinon fictionnelles. Il s'agit, d'une part, sous la plume des auteurs de la célèbre série inspirée de la mutinerie du Bounty, Charles Nordhoff et James Norman Hall de "The Englishman's story" qui constitue l'un des chapitres du recueil de nouvelles Faery Tales of the South Seas, publié en 1921, qui évoque et raconte la quête de $\mathrm{Howe}^{37}$. D'autre part, une nouvelle intitulée « Les Trois gendarmes de Pinaki » et signée par Pierre Luccin se réfère assez clairement à l'expédition de $1914^{38}$. Au cas assez virtuel du trésor de Pinaki et à l'engouement bien réel qu'il suscite peut s'appliquer la belle formule :

«L'étoffe dont les rêves sont faits.»

Il s'agit moins de se référer au vers final de la pièce de William Shakespeare, Richard III (1597) qu'à la dernière réplique du film Le Faucon Maltais (film de John Huston d'après Dashiell Hammett, avec Humphrey Bogart, 1941 ${ }^{39}$ ). Reconnaître et valoriser, c'est-à-dire donner le statut de fait, à ce qui n'est pas un fait - mais qui compte pourtant puissamment - ce n'est pas tourner le dos aux objectifs et aux principes d'exigence et de rigueur que l'École des chartes a contribué à défendre et à illustrer. Il s'agit, au contraire, de redéployer les mêmes outils critiques, alors que l'établissement de solides critères de validation et d'authentification de l'exactitude de l'information importe plus que jamais aujourd'hui ${ }^{40}$.

\section{BIBLIOGRAPHIE}

BERCÉ Yves-Marie, À la découverte des trésors cachés, Paris, Perrin, 2004.

BOUGAINVILLE Louis-Antoine de, Voyage autour du monde, Bideaux-Faessel, Paris, éd., Presses de l'Université Paris-Sorbonne, 2001.

BOURDIN Philippe et LE BRAS Stéphane (dir.), Les fausses nouvelles, un millénaire de bruits et de rumeurs dans l'espace public français, Centre d'Histoire Espaces et Cultures (CHEC), Presses de l'Université Blaise Pascal, Clermont-Ferrand, 2018.

BUTI Gilbert et HRODEJ Philippe, Dictionnaire des corsaires et des pirates, Paris, CNRS Éditions, 2013. CHEUNG Francis, Tahiti et ses îles (1919-1945), Étude d'une société coloniale aux antipodes de sa métropole, thèse de doctorat soutenue en 1983 à l'université Paris I-Sorbonne, L'Harmattan, 1998.

CHOUTEAU Marianne, FAUCHEUX Michel, THÉVENARD-NGUYEN Céline, « Technique et récit : Éléments d'une critique de la raison narrative » dans Triquet Éric (dir.), Le récit dans la médiation des sciences et des techniques, Culture \& Musées, $\mathrm{n}^{\circ} 18,2011$, p. 23-45.

CONTE Éric (dir.), Une histoire de Tahiti, des origines à nos jours, Au Vent des îles éditions, Tahiti, 2019. DANIELSSON Bengt et Marie-Thérèse, Papeete 1818-1990, Ch. Gleizal/Cobalt productions, Tahiti, 1990. DANIELSSON Bengt, Que s'est-il vraiment passé sur le Bounty? Haere Po éditions, Tahiti, 2013. 
DAMPIER William, Le Grand Voyage. Le tour du monde d'un flibustier, 1681-1691, Paris, Phébus, coll. « Littérature étrangère », 1993.

DARWIN Charles Robert, Geological Observations on Coral Reefs, Volcanic Islands and on South America, Londres, Smith, Elder \& co, 1851.

DIDEROT Denis, Supplément au Voyage de Bougainville dans CEuvres philosophiques, éd. P. Vernière, Garnier, 1964.

DE DECKKER Paul, Jacques-Antoine Moerenhout (1797-1879) - Ethnologue et consul, Au Vent des îles éditions, Tahiti, 1997.

DENING Greg, Mr Bligh's Bad Language: Passion, Power and Theatre on the Bounty, Cambridge University Press, 1994.

FRISBIE Robert Dean, Un âge d'or, Tahiti 1920-1923, trad. Henri Theureau, Ura éditions, Tahiti, 2017. GRAND Henri Vital (1871-1960), Tribulations à Tahiti et en Amérique, carnets d'une vie extraordinaire, présentés par sa petite-fille Simone Grand, éditions To'imata, Tahiti, 2012.

hamilton George, The Treasure of The Tuamotus, Londres, Stanley Paul \& Co. Ltd, 1939.

HAMON Renée, Amants de l'aventure, Paris, Flammarion, 1943.

JOULE François-Vincent et BEAUVOIS Jean-Louis, Petit traité de manipulation à l'usage des honnêtes gens, Grenoble, PUG, 2002.

LAUX Claire, Le Pacifique aux XVIII et XIX ${ }^{e}$ siècles, une confrontation franco-britannique : Enjeu colonial et rivalité géopolitique (1763-1914), Paris, Karthala, 2011.

LuCCIN Pierre, «Les Trois gendarmes de Pinaki », Les œuvres libres, $1^{\mathrm{er}}$ septembre 1950, $\mathrm{n}^{\circ} 52$, p. 123-172.

MARIC Tamara, Archéologie et traditions orales des atolls de Nukutavake, Vaira'atea et Pinaki, éd. numérique, Compte-rendu d'une campagne de recherches menée en mars 2009.

[URL : https://www.academia.edu/1007680/]

MAZELLIER Philippe (dir.), Le mémorial Polynésien, tome V, 1914-1939 (réalisé par Jean-Marie DalletBengt Danielsson-Philippe Mazellier), Papeete, Hibiscus éditions, 1977.

MU-LIEPMANN Véronique et TEHEI Vaihere, Papeete, Témoignages d'un autre temps, Commune de Papeete, 2008.

NORDHOFF Charles B. et HALL James Norman, Faery Tales of the South Seas, The Resnick Library of Worldwide Adventure, (1921) 2001, chap. XVII, “The Englishman's story”, p. 328-340.

O'REILlY Patrick et TEISSIER Raoul, Tahitiens, Répertoire biographique de la Polynésie française, Paris, Publications de la Société des Océanistes nº 36, Musée de L'Homme, 1975.

PAPIN Bernard, Sens et fonction de l'utopie tahitienne dans l'œuvre politique de Diderot, The Voltaire Foundation, Oxford, 1988.

VANANGA PIETRI Raymond, Pape'ete de jadis et naguère, Société des Études Océaniennes, 2017.

RENARD Jean-Bruno, « Introduction », Jean-Bruno RENARD (dir.), Rumeurs et légendes urbaines, Paris, Presses Universitaires de France, 2013, p. 3-8.

RICHARDS Rhys et LANGDON Robert, Tahiti and the Society Islands, Shipping Arrivals and Departures 1767 to 1852, Canberra, Pacific Manuscripts Bureau and Jean-Louis Boglio Maritime Books-The Australian National University, 2008. 
STEVENSON Robert-Louis, CEuvres, T. 1, L'T̂le au trésor..., La Pléiade, Paris, Gallimard, 2001.

TCHERKÉZOFF Serge, « La Polynésie des vahinés et la nature des femmes : une utopie occidentale masculine », Clio, femmes, genre, histoire, 22, 2005.

TOULLELAN Pierre-Yves, « Les Colons et l'agriculture à Tahiti dans la seconde moitié du XIX siècle ", Journal de la Société des Océanistes, $\mathrm{n}^{\circ}$ 74-75, 1982.

TOULLELAN Pierre-Yves, Tahiti colonial (1860-1914), Paris, Publications de la Sorbonne, 1987.

\section{NOTES}

1. https://bibliotheque-numerique.diplomatie.gouv.fr/ark:/12148/bpt6k451943k/ f1.image.texteImage : Journal des débats politiques et littéraires, 8 avril 1859.

2. Arch. terr. Polynésie française, Fonds $48 \mathrm{~W}$, lettre du gouverneur au ministre, 9 janvier 1920.

3. G. Hamilton, The Treasure of The Tuamotus, p. 122. Richards et Langdon, Tahiti and the Society Islands, Shipping Arrivals and Departures 1767 to 1852, p. 96.

4. P. O’Reilly et R. Teissier, Tahitiens, Répertoire biographique de la Polynésie française, p. $264,329,528$.

5. Contrat retranscrit dans Ph. Mazellier, Le Mémorial Polynésien, p. 265.

6. Rapport de gendarmerie du brigadier Fromentin (févr. 1914) publié par la capitaine P. Bagarie, «Le Trésor de Pinaki », p. 36-43. Arch. terr. Polynésie française, Fonds 48W, Lettre $\mathrm{du}$ procureur Simoneau, remplaçant du gouverneur Fawtier à partir du 21 janvier 1914 au chef de détachement, 24 janvier 1914 ; lettre du procureur Simoneau, remplaçant du gouverneur Fawtier au chef de service, 24 janvier 1914; lettre au ministre du gouverneur Fawtier, 7 février 1914.

7. Journal Officiel des E.F.O., 1923, p. 338.

8. V. Mu-Liepmann, Papeete, témoignage d'un autre temps, p.171-5, F. Gallon-Juventin, Tahiti de ma jeunesse 1930-1950, p. 7-8.

9. Témoignage d'André Juventin (56 ans après les faits), dans Ph. Mazellier, Mémorial polynésien, p. 271.

10. F. Cheung, Tahiti et ses îles (1919-1945), Étude d'une société coloniale aux antipodes de sa métropole, p. 273-8.

11. Témoignage d'André Juventin (56 ans après les faits), dans Ph. Mazellier, Mémorial polynésien p. 272-273.

12. Arch. terr. Polynésie française, Fonds $48 \mathrm{~W}$, lettre du gouverneur au ministre, 9 janvier 1920.

13. G. Hamilton, The Treasure of The Tuamotus, p. 144, 162. Témoignage d'André Juventin (56 ans après les faits), dans Ph. Mazellier, Mémorial polynésien p. 273.

14. T. Maric, Archéologie et traditions orales des atolls de Nukutavake, Vaira'atea et Pinaki, p. $40-49$.

15. Arch. terr. Polynésie française, fonds du gouverneur, 48W, «Étrangers »: Décret d'expulsion de Charles Edouard Howe, 24 février 1928. G. Hamilton, The Treasure of The Tuamotus, p. 163-4. 
16. http://www.oceantreasures.org/pages/content/world-of-shipwrecks/theperuvian-lost-treasures.html: The lost treasures of the Peruvian Church par Pascal Kainic et Ken Matthews.

https://www.tahitiheritage.pf/tresor-pisco-tuamotu/.

17. https://www.tntv.pf/replay/documentaires/pinaki-partie-1/

https://www.tntv.pf/replay/documentaires/pinaki-partie-2/

18. J-B. Renard, Rumeurs et légendes urbaines, p. 3-8.

19. T. Maric, Archéologie et traditions orales des atolls de Nukutavake, Vaira'atea et Pinaki, p. 40-49.

20. Le Voyage autour du Monde de Louis-Antoine de Bougainville (1 ere édition 1771; $2^{2}$ édition 1772). Le Supplément au voyage de Bougainville de Denis Diderot (rédaction 1772 puis $1778-9$; publication 1796).

21. W. Dampier, Le Grand Voyage. Le tour du monde d'un flibustier, 1681-1691, G. Buti et Ph. Hrodej, Dictionnaire des corsaires et des pirates, p. 193.

22. B. Danielsson, Que s'est-il vraiment passé sur le Bounty?, G. Dening, Mr Bligh's Bad Language: Passion, Power and Theatre on the Bounty, p. 357-8.

23. E. Conte (dir.), Une histoire de Tahiti, p. 145-248.

24. P. Toulellan, Tahiti colonial, p. 254-6.

25. C. Laux, Le Pacifique aux XVIII et XIX siècles, une confrontation franco-britannique: Enjeu colonial et rivalité géopolitique (1763-1914), p. 153-164.

26. P. Y. Toullelan, «Les Colons et l'agriculture à Tahiti dans la seconde moitié du XIX ${ }^{e}$ siècle », p. 213-225.

27. P. Y. Toullelan, Tahiti colonial (1860-1914), p. 273.

28. Y.-M. Bercé, À la découverte des trésors cachés du XVI siècle à nos jours, p. 54.

29. P. de Deckker, Jacques-Antoine Moerenhout (1797-1879) - Ethnologue et consul, p. 28-31, 78-81.

30. R. V. Pietri, Papeete de jadis et naguère, p. 80-1.

31. M. Chouteau, M. Faucheux, C. Thévenard-Nguyen, «Technique et récit: Éléments d'une critique de la raison narrative », p. 25.

32. Notamment Ch. Darwin, Geological Observations on Coral Reefs, Volcanic Islands and on South America, p. 96. Ph. Mazellier, Mémorial polynésien, p. 267.

33. L'̂́le au Trésor (titre original : Treasure Island) film muet $35 \mathrm{mn}$, réalisation : Maurice Tourneur, scénario: Jules Furthman, production: Maurice Tourneur, distribution: Famous Players-Lasky Corporation, pays d'origine : États-Unis, durée : 76 minutes, date de sortie : 11 avril 1920.

34. Tabu, A story of the South Seas, film muet, réalisation: F. W. Murnau, pays d'origine : États-Unis, format : noir et blanc, production : Paramount Pictures, durée : $84 \mathrm{mn}$, date de sortie : 1931 .

35. Treasure Island, film parlant, réalisation: Victor Fleming, production: MetroGoldwyn-Mayer, pays d'origine : États-Unis, Format : Noir et Blanc, durée : 109 minutes, date de sortie : 1934, catalogue de l'American Film Institute.

36. Fr.-V. Joule et J.-L. Beauvois, Petit traité de manipulation à l'usage des honnêtes gens, p. 53-68 et 97-112; p. 171-193 en particulier. 
37. Ch. Nordhoff et J.-N. Hall, “The Englishman's story”, p. 328-340.

38. P. Luccin, «Les Trois gendarmes de Pinaki », p. 123-172.

39. https://www.afi.com.

40. Ph. Bourdin et S. Le Bras (dir.), Les fausses nouvelles, un millénaire de bruits et de rumeurs dans l'espace public français, p. 195-206.

\section{RÉSUMÉS}

Entre 1914 et 1994, la conviction qu'un trésor était enfoui dans l'un des atolls de l'archipel polynésien des Tuamotu fut assez forte pour motiver l'organisation d'expéditions au départ de Tahiti. Tout reposait sur les dires d'un personnage louche se rapportant au vol d'une colossale somme d'or et autres biens précieux dans un couvent péruvien vers 1850 . Il jouait ainsi sur les lieux communs traditionnels de l'imaginaire de la piraterie. Ses dires tenaient aussi aux fantasmes typiques des îles du Pacifique sud. Or toutes les tentatives pour retrouver ce trésor restèrent sans résultants probants et toute l'histoire relève certainement de l'escroquerie. Il s'agit d'étudier, d'une part, les processus de manipulation qui lui conféraient crédibilité et attractivité et, d'autre part, de considérer en quoi elle est un révélateur du cadre colonial des Établissements Français d'Océanie (E.F.O) précédant celui de l'actuelle Polynésie française.

\section{AUTEUR}

\section{VÉRONIQUE DORBE-LARCADE}

Maître de conférences HDR en histoire moderne, université de la Polynésie française 


\section{Du pouvoir fantasmé}




\title{
La frontière politique : réalités géopolitiques complexes ou représentations idéologiques partisanes à partir de l'exemple de la vallée du Rhône en Révolution
}

\author{
Nicolas Soulas
}

Club des Jacobins d'Orange, 26 avril 1791. Alors que la guerre civile fait rage dans le Comtat, les clubistes orangeois rédigent une adresse à l'Assemblée constituante pour dénoncer les incursions de bandes comtadines sur son territoire. Dans ce texte, les Orangeois rappellent leur situation géopolitique particulière : « enclavés dans une terre étrangère ${ }^{1} » . A u$ cours du printemps 1797 , soit six ans plus tard, dans une lettre adressée au ministre de l'Intérieur, la municipalité brosse un tableau très sombre de la situation politique d'Orange, présentée comme étant « enclavée dans un pays en proye [sic] à tous les excès du fanatisme ${ }^{2}$ ». Ces deux adresses, rédigées dans des contextes politiques différents, sont particulièrement intéressantes quant à la perception que les acteurs politiques ont de leur environnement proche. Les Orangeois font référence à deux reprises à leur situation d'enclavement. Toutefois, la réalité est-elle conforme à ces discours ou ces derniers ne traduiraient-ils qu'une simple vue de l'esprit, entretenue et renforcée par la constitution d'un nouvel imaginaire politique durant la Révolution française ? En effet, si en avril 1791, l'ancienne principauté d'Orange est bel et bien encerclée par le Comtat Venaissin, au printemps 1797, il n'existe plus aucune terre étrangère dans le département du Vaucluse. En somme, les deux adresses font clairement allusion à la thématique de la frontière politique, qu'elle soit formelle, dans le cas de la frontière internationale séparant le royaume de France du Comtat pontifical en 1791, ou beaucoup plus informelle, traduisant dans ce cas l'existence d'un hiatus entre une géographie politique complexe et les représentations idéologiques et partisanes qu'en ont les contemporains. 
2 Sous la Révolution française, la nationalisation de la vie politique locale bouleverse les échiquiers politiques et favorise, par la construction de positionnements idéologiques antagonistes, la constitution de vastes lignes de fractures politiques ${ }^{3}$. Dans les espaces les plus politisés comme l'Ouest de la France ${ }^{4}$ ou le Midi ${ }^{5}$, l'émergence et l'affirmation d'options politiques concurrentielles débouchent sur la formation précoce de frontières politiques $^{6}$, séparant, de manière purement informelle, des espaces politiques diamétralement opposés, et perçus comme tels par leurs acteurs.

3 Parce qu'elle connaît une vie politique locale mouvementée, la vallée du Rhône, entendue comme le bassin fluvial couvrant sept départements ${ }^{7}$ des faubourgs de Lyon à Arles, s'avère un laboratoire idéal pour appréhender le processus de création et d'enracinement des frontières politiques dès les premiers mois de la Révolution française. En effet, la radicalisation précoce des options politiques, inhérente à la dégradation de la conjoncture politique au cours de l'année 1790, contribue à l'élaboration d'une géographie politique à géométrie variable qui s'enracine au gré des différents scrutins. Appréhender avec précision la constitution de lignes de fractures politiques nécessite de recourir à une approche micro-analytique ${ }^{8}$, au plus proche des vécus et des pratiques. En effet, c'est à l'aune des élections municipales, à travers le profil politique des élus et de leur positionnement idéologique lors des crises nationales, comme la crise «fédéraliste » de l'été 1793, ou plus régionales, comme l'affaire des camps de Jalès, que les fractures politiques se révèlent. Pour ce faire, il convient de s'immerger dans les affaires politiques locales et de tenter de renseigner le positionnement politique des individus9.

4 La tâche est complexe, car classer politiquement des acteurs en l'absence de partis politiques officiels requiert une extrême prudence quant à la manipulation d'étiquettes politiques ${ }^{10}$ souvent peu perspicaces et parfois très fluctuantes ${ }^{11}$. Toutefois, le recours à la prosopographie permet de surmonter, en partie, cet écueil et d'esquisser une classification politique acceptable, élaborée à partir de trajectoires individuelles variées : arrestation en l'an II, désarmement en l'an III, départ en émigration, adhésion ou refus d'adhérer à un club politique, pétitions, etc. Ces catégorisations politiques prennent tout leur sens au sein d'une mise en perspective multiscalaire ${ }^{12}$, offrant une meilleure appréciation de la question de la constitution des frontières politiques.

5 Enfin, la grande diversité des sources convoquées (pétitions, délibérations municipales, adresses, etc.) permet d'approfondir l'analyse en lui adjoignant une dimension sémantique. Les discours des contemporains et le vocabulaire utilisé pour dépeindre les arènes politiques rhodaniennes renseignent davantage sur l'espace perçu que sur les réalités politiques vécues. En s'inscrivant au cœur de stratégies partisanes visant à susciter l'intervention des pouvoirs extérieurs, ces représentations participent à la création d'un nouvel imaginaire politique ${ }^{13}$ qui, en amplifiant la complexité de la géographie politique rhodanienne, entretient dans les esprits une vision fantasmée de la frontière politique.

\section{Une géographie politique rhodanienne très complexe}

6 Comme dans d'autres espaces méridionaux, notamment le sud-est de la France, le climat politique régional se dégrade rapidement au cours de l'année 1790. Des premières frictions apparaissent lors de la découverte de différents complots contrerévolutionnaires qui éclosent sur les marges du couloir rhodanien. Les échos de la 
bagarre de Nîmes ${ }^{14}$ (juin 1790) et surtout des deux premiers camps de Jalès ${ }^{15}$ (été 1790 , février 1791) fissurent la concorde politique et alimentent les rumeurs de "complots aristocrates ${ }^{16}$ ", créant un climat anxiogène de suspicion, propre à radicaliser les esprits. Ces tensions sont amplifiées dans la partie méridionale de la vallée du Rhône par la crise comtadine ${ }^{17}$ qui affecte la stabilité des territoires français limitrophes ${ }^{18}$. Un Comtat septentrional, devenu une terre de conservatisme politique et d'opposition à l'annexion à la France, se regroupant au sein de l'Union de Sainte-Cécile, s'oppose à un Comtat méridional, chapeauté par Avignon et réclamant l'union avec la France. Les deux « partis » qui s'opposent inondent le sud de la France de discours et d'écrits qui sont relayés jusqu'à Paris. Les patriotes, pro-français, triomphent dans cette guerre d'imprimés et se montrent plus percutants, en amalgamant notamment leurs adversaires avec les différents foyers de la contre-révolution méridionale. Ce faisant, ils renforcent dans l'imaginaire des populations le sentiment d'évoluer dans un environnement hostile. Ces peurs ${ }^{19}$, qui ne sont pas totalement infondées, accentuent la radicalisation des patriotes, surtout dans les zones ayant noué d'étroits contacts avec le «parti» pro-français. Dans toute une partie de la vallée du Rhône, l'intensité du schisme religieux, créé par la Constitution civile du clergée ${ }^{20}$, et l'émergence de sociétés politiques de combat, cherchant à s'affirmer dans un milieu qui leur est défavorable et défendant ardemment la Révolution, parachèvent la dégradation du climat politique régional. Les tensions sont particulièrement vives et les affrontements entre factions récurrents.

7 Les divisions des premières années de la Révolution favorisent l'émergence de comportements politiques antagonistes, qu'une étude très fine des scrutins municipaux permet de cartographier. Comme l'illustrent les cartes (fig. 1), ces attitudes politiques s'enracinent. Dans le sillon rhodanien, l'existence de positionnements idéologiques divergents donne naissance dès 1791 à une géographie politique complexe où cohabitent en contiguïté régionale des espaces politiques diamétralement opposés ${ }^{21}$. 
Fig. 1. - Géographie politique du couloir rhodanien (1791).

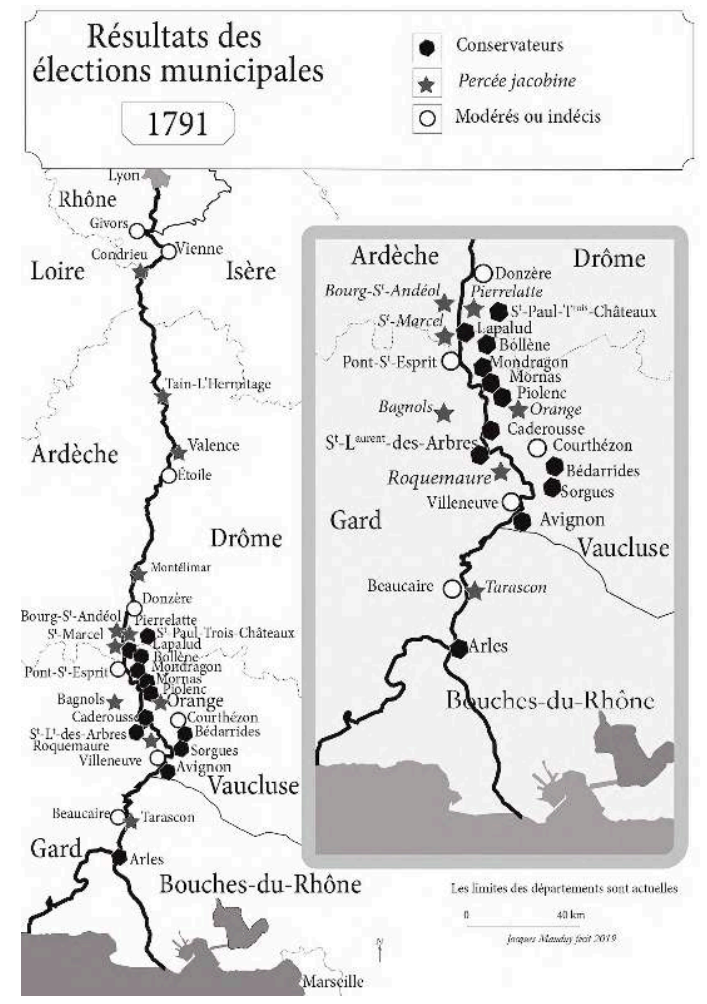

Doc. N. Soulas.

8 L'ancien Comtat rhodanien, de Bollène à Piolenc, apparaît comme un espace homogène de conservatisme politique ${ }^{22}$, où les populations confirment des élites politiques réactionnaires s'étant signalées par leur conservatisme et leur opposition à Avignon au cours de la guerre civile. Par ailleurs, les patriotes de l'ancien Comtat méridional sont désavoués par les électeurs qui placent des conservateurs à la tête des pouvoirs locaux, notamment en raison de leur compromission dans les massacres de la Glacière en octobre $1791^{23}$. À l'exception de la commune d'Arles, les foyers conservateurs se trouvent dans un rayon géographique proche de l'ancien Comtat. La proximité du Comtat se répercute dans la constitution des options politiques locales, agissant comme un véritable poison pour les territoires environnants qu'il contamine et qu'il déstabilise, ou produisant l'effet inverse, agissant comme un repoussoir, favorisant la constitution précoce de citadelles jacobines, comme Orange ou Bagnols. La guerre civile comtadine a profondément affecté la vie politique orangeoise. Le club des jacobins orangeois, accueillant à sa tribune de nombreux patriotes comtadins, ressort radicalisé de ce conflit périphérique. Les autres bastions jacobins éclosent également proches d'espaces contre-révolutionnaires comme Tarascon, près d'Arles, et Bourg-SaintAndéol dans les environs immédiats de la plaine de Jalès. Enfin, d'autres municipalités ont un profil plus complexe. Cherchant une voie médiane entre le conservatisme et la radicalisation professée par certains patriotes, elles se distinguent par des prises de position très modérées (Vienne, Pont-Saint-Esprit, etc.) ${ }^{24}$. L'existence d'un tel courant n'est possible que dans les localités qui n'ont pas été affectées par la crise religieuse de 1791 ou dont le modèle communautaire résiste mieux aux fractures révolutionnaires. Le recours à la micro-analyse et au jeu d'échelles permet d'esquisser avec finesse les contours de cette géographie politique complexe, variant d'un canton à un autre, ou 
entre plusieurs localités d'un même canton. Ainsi, dans le district d'Orange, le chef-lieu apparaît comme une citadelle jacobine encerclée par des campagnes conservatrices. Les frontières politiques qui se constituent à la fin de l'année 1791 sont à la fois le fruit d'un héritage historique et des divisions des débuts de la Révolution française. C'est particulièrement net pour le haut Comtat rhodanien qui se signalait déjà par son attitude conservatrice à la veille de la Révolution, phénomène amplifié par son incorporation au royaume de France. Du reste, une frontière politique interne et totalement informelle se substitue à la frontière institutionnelle qui séparait Avignon et le Comtat lors de l'annexion de 1791.

9 Comme le suggère le scrutin de 1795 (fig. 2), la Révolution fige les comportements politiques. Au-delà de quelques variations locales que nous ne saurions expliquer ici ${ }^{25}$, les mêmes tendances politiques se retrouvent sur les deux cartes: l'ex-Comtat rhodanien reste la terre de prédilection du conservatisme politique tandis que dans certaines localités, comme Orange ou Valence, s'est forgée une identité jacobine très précoce qui continue de s'affirmer, quelle que soit la conjoncture politique.

Fig. 2. - Géographie politique du couloir rhodanien (1795).

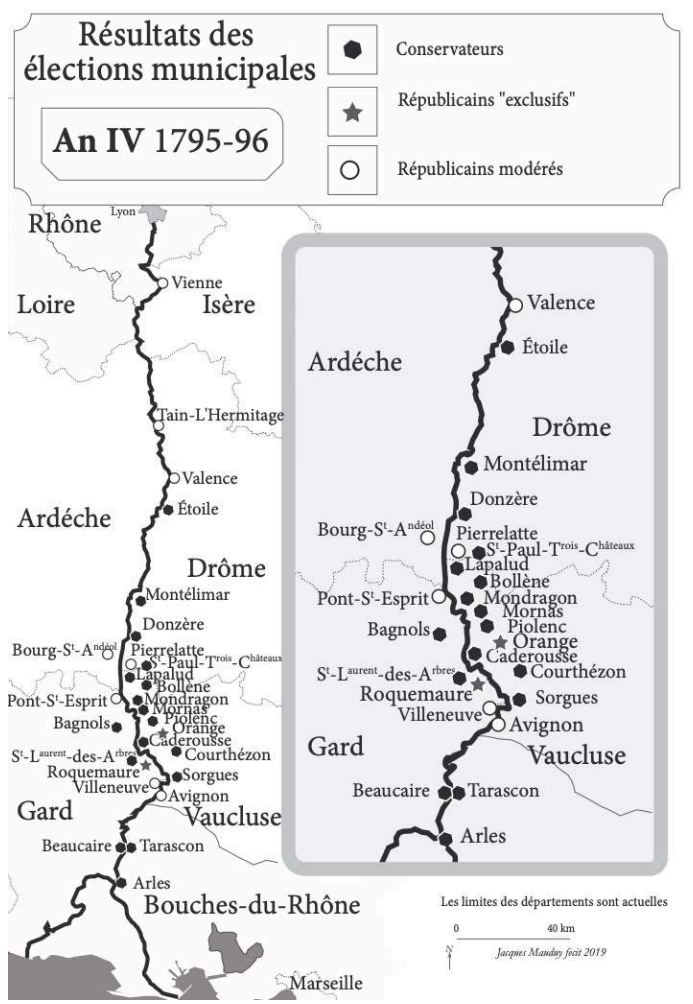

Doc. N. Soulas

Si l'existence d'antagonismes politiques particulièrement tranchés favorise la constitution de lignes de fractures politiques importantes, les frontières politiques procèdent en partie de constructions mentales, alimentées par un nouvel imaginaire politique partisan, livrant une vision déformée et partiale de la réalité. Une lecture attentive de différentes sources (adresses, discours prononcés à la tribune des clubs politiques, correspondances) atteste cette perception partisane, contribuant à rendre palpables des discontinuités politiques purement informelles. 


\section{Des frontières politiques fantasmées?}

11 Les représentations de la frontière politique s'appuient, certes, sur une géographie politique complexe mais procèdent également beaucoup des discours produits par les acteurs. Depuis 1790, journalistes patriotes, clubistes et administrations locales déforment et exagèrent la complexité de la géopolitique régionale. S'il existe une réelle menace contre-révolutionnaire dans le Midi, celle-ci est amplifiée dans les discours par le recours à une nouvelle terminologie révolutionnaire standardisée qui ne reflète pas vraiment la réalité politique vécue. Ainsi, l'expression «nouvelle Vendée ${ }^{26}$ », faisant référence au conflit vendéen de 1793, vise à stigmatiser des pôles conservateurs, même s'ils ne se revendiquent jamais eux-mêmes comme sympathisants de la cause vendéenne, et encore moins comme royalistes. Lieu commun du discours patriote à partir de $1793^{27}$, la référence à la Vendée constitue un leitmotiv important de la rhétorique des républicains «exclusifs » méridionaux ${ }^{28}$. Elle donne plus de force et de crédit aux adresses et aux discours tandis qu'elle ancre dans l'imaginaire des populations locales, et du pouvoir central, la vision d'un espace politique extrêmement fragmenté aux frontières politiques palpables.

12 Cette construction mentale est alimentée par un très fort sentiment d'isolement et d'insécurité permanente, partagé et relayé par de nombreux patriotes méridionaux dans leurs adresses et leurs discours. Le phénomène est particulièrement lisible à Orange, fief patriote de la première heure, encerclé par des villages conservateurs qui lui sont hostiles. Les partisans de la Révolution, notamment comtadins, chassés de leur village, viennent régulièrement y trouver refuge, en 1791-1792 ou entre 1795 et 1798. Derrière le conflit traditionnel opposant villes et campagnes, ces errances politiques renforcent dans les imaginaires l'existence de frontières politiques tangibles. Les productions écrites et les discours des exilés politiques, comme des patriotes orangeois, sont particulièrement éclairants à ce sujet. Bien que la référence à la frontière ne soit jamais mentionnée dans les sources, ces hommes ressentent clairement l'opposition des villages voisins comme une menace planant dangereusement sur la ville et l'existence de deux mondes politiques antagonistes. Outre leur aspect anxiogène, les références à l'isolement ou à l'encerclement traduisent l'existence d'un hiatus important entre une géopolitique régionale et la façon dont elle est vécue et perçue par des patriotes minoritaires dans un environnement hostile.

Ces représentations s'inscrivent dans la construction politique du Midi $^{29}$, perçu par le pouvoir central comme la terre d'élection de la contre-révolution, même si dans les faits c'est loin d'être toujours le cas. En effet, les violences qui ensanglantent le Midi de la France après thermidor alimentent l'image biaisée et partiale de départements méridionaux gangrenés par le royalisme. Les patriotes "exclusifs", déchus des pouvoirs locaux entre l'automne 1794 et le printemps 1795, entretiennent cet imaginaire politique dans leurs adresses envoyées au gouvernement. Dans leurs discours, toutes les vexations qu'ils subissent sont volontiers présentées comme étant l'œuvre d'une chimérique Compagnie de Jésus, composée d'agents royalistes actifs visant à rétablir la monarchie. Or, une telle organisation n'a, selon toute vraisemblance, jamais existé. En réalité, les historiens ont clairement montré que ces violences, bien réelles et particulièrement sanglantes, constituent surtout la manifestation, dans leur dimension la plus tragique, de conflits interpersonnels que la Révolution a politisés ${ }^{30}$. Toutefois, en banalisant cette expression, les républicains « exclusifs » entretiennent 
une vision déformée des réalités politiques locales, où les règlements de compte politiques sont systématiquement assimilés à de la contre-révolution ou au royalisme, tout en diabolisant leurs adversaires.

L'entretien dans l'imaginaire collectif d'une vision déformée et très partisane des lignes de fractures politiques constitue l'un des volets des stratégies politiques de patriotes «exclusifs" marginalisés, au plus fort d'une conjoncture qui ne leur est guère favorable. En offrant une vision caricaturale des dynamiques politiques rhodaniennes, les populations locales cherchent à attirer l'attention du pouvoir central dans l'optique de bénéficier de son arbitrage, sinon de son soutien pour triompher de leurs adversaires. Ils ont, par conséquent, tout intérêt à alimenter cette construction mentale fantasmée. Cette stratégie conforte, vue de Paris, une conception très manichéenne des arènes politiques locales, opposant les républicains à des adversaires diabolisés et discrédités par des étiquettes politiques, totalement inconciliables avec l'idéal républicain. L'objectif est pleinement atteint. Pour le pouvoir central, la vallée du Rhône, et plus largement le Midi de la France, passe pour une terre de non-droits pour les patriotes, où les règlements de compte politiques, portant la marque du royalisme, sont systématiquement assimilés à la contre-révolution triomphante. En dramatisant le potentiel de nuisance de leurs adversaires, en grossissant le nombre de victimes, en exagérant les violences subies, les républicains délégitiment leurs opposants mais surtout, en attirant l'attention du gouvernement, ces manœuvres permettent aux républicains "exclusifs" de rétablir l'équilibre politique dans des espaces où les scrutins ne leur sont pas favorables. Ainsi à Bollène, cité vauclusienne très marquée par les affrontements politiques depuis 1790 , la municipalité réactionnaire conduite par le notaire Pelliard est destituée par le représentant Fréron en janvier $1796^{31}$. La nouvelle équipe municipale se recrute parmi les anciens cadres de l'an II, à l'origine d'une adresse au Directoire, accusant la municipalité de collusion avec les ennemis de la Révolution.

En élargissant la sphère politique à un plus grand nombre d'acteurs que par le passé et en nationalisant la vie politique locale, la Révolution française favorise l'apparition de profondes lignes de fractures politiques dans le sillon rhodanien dès 1791. À la fois manifestations de la rupture précoce du consensus politique et expressions de la vitalité des options politiques méridionales, ces frontières politiques s'enracinent face à la radicalisation du processus révolutionnaire. Cette géographie politique contrastée met en évidence l'existence d'une acculturation politique divergente, traduisant la multiplicité des vécus révolutionnaires et l'inégale réaction face aux nouvelles règles du jeu imposées par le pouvoir central. Par ailleurs, la multiplication des adresses et des discours, amplifiant et déformant les luttes politiques rhodaniennes, contribue à forger une nouvelle représentation politique du couloir rhodanien, et plus largement du Midi de la France. Dans cet espace géographique de très forte conflictualité, les frontières politiques apparaissent tout autant comme étant le fruit d'une géopolitique contrastée que les projections fantasmées de représentations politiques, en partie entretenue et alimentée par des stratégies partisanes. La virulence des antagonismes et les représentations déformées qui en résultent, véhiculées par les clubs ou par les adresses, contribuent à forger un imaginaire politique manichéen, propre à générer de la radicalisation politique en entretenant un climat politique anxiogène. Ce faisant, ces 
représentations fantasmagoriques contribuent à enraciner dans l'imaginaire collectif l'image biaisée et partisane d'un espace géographique perçu, à tort, par le pouvoir central ou par certaines populations locales, comme un bastion de la contre-révolution et du royalisme. Cette illusion perdure dans les esprits au moins jusqu'à la révolution de $1830^{32}$.

\section{BIBLIOGRAPHIE}

BERCÉ Yves-Marie, (dir.), Les autres Vendées. Les contre-révolutions paysannes au XIXe siècle, La Rochesur-Yon, Éditions du CVRH, 2013.

BoIs Paul, Paysans de l'ouest. Des structures économiques et sociales aux options politiques depuis l'époque révolutionnaire dans la Sarthe, Paris, Flammarion, 1971.

CLAY Stephen, «Les réactions du Midi : conflits, continuités et violences », Annales historiques de la Révolution française, 345, 2006, p. 55-91.

CRAIUTU Aurelian, A virtue for courageous Minds? Moderation in french political thought, 1748-1830, Princeton, Princeton University Press, 2012.

DUPORT Anne-Marie, Journées révolutionnaires à Nîmes, Paris, Éditions Chambon, 1988.

GUILHAUMOU Jacques, « Le discours de salut public d'Hébert au Club des jacobins le 21 juillet 1793. Une rhétorique de la minorité politique », dans Peyrard Christine (dir.), Minorités politiques en Révolution (1789-1799), Aix-en-Provence, Presses universitaires de Provence, 2007, p. 43-59.

HUARD Raymond, La naissance du parti politique en France, Paris, Presses de la Fondation nationale des sciences politiques, 1996.

L'invention du Midi. Représentations du Sud pendant la période révolutionnaire, dossier préparé par Philippe Martel dans Amiras/repères occitans, Aix-en-Provence, Edisud, 1987.

JOUVENEL François de, «Les camps de Jalès (1790-1792), épisodes contre-révolutionnaires ? ", Annales historiques de la Révolution française, $\mathrm{n}^{\circ} 337$, juillet-septembre 2004, p. 1-20.

LAMIZET Bernard, L'imaginaire politique, Paris, Hermes-Science Lavoisier, 2012.

LAPIED Martine, Le Comtat et la Révolution française. Naissance des options collectives, Aix-en-Provence, Publications de l'Université de Provence, 1996.

LEPETIT Bernard, « De l'échelle en histoire », dans Jeux d'échelles. La micro-analyse à l'expérience. Textes rassemblés par Jacques Revel, Paris, Gallimard/Le Seuil, 1996, p. 71-94.

MARTIN Jean-Clément, Blancs et bleus dans la Vendée déchirée, Paris, Gallimard, 2001.

MOULINAS René, Histoire de la Révolution d'Avignon, Avignon, Aubanel, 1986.

MOULINAS René, Les massacres de la Glacière : enquête sur un crime impuni, Avignon 16-17 octobre 1791, Aix-en-Provence, Édisud, 2003.

PEYRARD Christine, « Partis, factions, lignées et pouvoir local », Rives nord-méditerranéennes, $\mathrm{n}^{\circ} 1$, 1998, p. 19-23. 
REVEL Jacques, « Micro-analyse et construction du social », dans Jeux d'échelles. La micro-analyse à l'expérience. Textes rassemblés par Jacques Revel, Paris, Gallimard/Le Seuil, 1996, p. 15-36.

SотTOCASA Valérie, Mémoires affrontées. Protestants et catholiques face à la Révolution dans les montagnes du Languedoc, Rennes, Presses universitaires de Rennes, 2004.

SOULAS Nicolas, Révolutionner les cultures politiques : l'exemple de la vallée du Rhône (1750-1820), Avignon, Éditions universitaires d'Avignon, 2020, 549 p.

SOULAS Nicolas, «Conflits, circulations et construction du politique. Le Midi de la France au prisme de la crise comtadine (1790-1792) », Annales historiques de la Révolution française, $\mathrm{n}^{\circ} 398$, septembre-décembre 2019, p. 47-68.

TACKETT Timothy, La Révolution, l'Église, la France. Le serment de 1791, Paris, Éditions du Cerf, 1986.

TACKETT Timothy, « La Grande Peur et le complot aristocratique sous la Révolution française », Annales historiques de la Révolution française, $\mathrm{n}^{0}$ 335, janvier-mars 2004, p. 1-17.

TORT Olivier, « L'image du Midi sous la Restauration : variations autour du tempérament méridional », Annales du Midi, tome 124, octobre-décembre 2012, p. 436-453.

voveLLE Michel, « Midi rouge, Midi blanc : une problématique », Provence historique, 37, 148, 1987, p. 337-347.

VOVELLE Michel, « Massacreurs et massacrés. Aspects sociaux de la contre-révolution en Provence après thermidor », dans Lebrun François, Dupuy Roger (dir.), Les résistances à la Révolution, Paris, Imago, 1987, p. 141-150.

VOVELLE Michel, La découverte de la politique. Géopolitique de la Révolution française, Paris, La Découverte, 1992.

WAHNICH Sophie, Les émotions, la Révolution française et le présent. Exercices pratiques de conscience historique, Paris, CNRS, 2009.

\section{NOTES}

1. Médiathèque Ceccano d'Avignon, ms. 2523, adresse du club des Jacobins d'Orange, 26 avril 1791.

2. Arch. nat., F1CIII Vaucluse 1, lettre de la municipalité d'Orange au ministre de l'intérieur, 7 floréal an V.

3. N. Soulas, Révolutionner les cultures politiques : l'exemple de la vallée du Rhône (1750-1820).

4. P. Bois, Paysans de l'ouest. Des structures économiques et sociales aux options politiques depuis l'époque révolutionnaire dans la Sarthe, 1971 ; J.-C. Martin, Blancs et bleus dans la Vendée déchirée, 2001.

5. V. Sottocasa, Mémoires affrontées. Protestants et catholiques face à la Révolution dans les montagnes du Languedoc, 2004.

6. M. Vovelle, La découverte de la politique. Géopolitique de la Révolution française, 1992.

7. Rhône, Isère, Ardèche, Drôme, Vaucluse, Gard, Bouches-du-Rhône.

8. J. Revel, « Micro-analyse et construction du social », p. 15-36. 
9. Dans le cadre de notre doctorat, nous avons constitué un échantillon d'un peu plus de 5000 détenteurs de fonctions locales entre 1750 et 1820 . Il est d'une aide capitale pour bâtir cette enquête.

10. C. Peyrard, «Partis, factions, lignées et pouvoir local », p. 19-23.

11. R. Huard, La naissance du parti politique en France.

12. B. Lepetit, « De l'échelle en histoire », p. 71-94.

13. B. Lamizet, L'imaginaire politique.

14. La fracture religieuse, sublimée par les tensions économiques et sociales, conduit à un affrontement violent entre catholiques et protestants pour le contrôle du pouvoir local du 13 au 15 juin 1790 : A.-M. Duport, Journées révolutionnaires à Nîmes.

15. Il s'agit de rassemblements de gardes nationales, chapeautés par des contrerévolutionnaires notoires, qui se tiennent dans le château de Banne, dans la plaine ardéchoise de Jalès : F. de Jouvenel, « Les camps de Jalès (1790-1792), épisodes contrerévolutionnaires?", p. 1-20.

16. T. Tackett, «La Grande Peur et le complot aristocratique sous la Révolution française ", p. 1-17.

17. Une partie des Comtadins cherchent à se détacher de la domination pontificale pour des raisons économiques importantes tandis que d'autres rejettent l'union à la France révolutionnaire et veulent, au contraire, rester sous l'autorité romaine, car cette dernière apporte de considérables avantages: pas de milice, peu d'impôts, etc. Ces divisions débouchent sur un conflit politique majeur entre l'été 1790 et l'été 1791 : R. Moulinas, Histoire de la Révolution d'Avignon; M. Lapied, Le Comtat et la Révolution française. Naissance des options collectives.

18. N. Soulas, « Conflits, circulations et construction du politique. Le Midi de la France au prisme de la crise comtadine (1790-1792)», p. 47-68.

19. S. Wahnich, Les émotions, la Révolution française et le présent. Exercices pratiques de conscience historique.

20. T. Tackett, La Révolution, l'Église, la France. Le serment de 1791.

21. M. Vovelle, « Midi rouge, Midi blanc : une problématique », p. 343.

22. Ont été classés sous cette étiquette les individus qui adoptent des positionnements réactionnaires: allant de la contre-révolution au soutien du clergé réfractaire, à l'hostilité au jacobinisme, etc.

23. À la suite du meurtre du patriote Lescuyer, les révolutionnaires les plus radicaux arrêtent une soixantaine de personnes qui sont enfermées dans le Palais. Dans la nuit du 16 au 17 octobre 1791, la plupart des prisonniers sont massacrés et jetés dans la tour de la glacière. Le massacre connaît un fort retentissement. Les modérés sont offusqués par la violence tandis que les radicaux justifient pleinement son emploi. R. Moulinas, Les massacres de la Glacière : enquête sur un crime impuni, Avignon 16-17 octobre 1791.

24. A. Craiutu, A virtue for courageous Minds? Moderation in french political thought, 1748-1830.

25. Le lecteur trouvera de plus amples explications dans notre thèse de doctorat.

26. Y.-M. Bercé (dir.), Les autres Vendées. Les contre-révolutions paysannes au XIXe siècle.

27. J. Guilhaumou, «Le discours de salut public d'Hébert au Club des Jacobins le 21 juillet 1793. Une rhétorique de la minorité politique », p. 43-59. 
28. Cette étiquette politique, forgée par les contemporains, désigne les patriotes les plus « radicaux».

29. L'invention du Midi. Représentations du Sud pendant la période révolutionnaire.

30. M. Vovelle, «Massacreurs et massacrés. Aspects sociaux de la contre-révolution en Provence après thermidor», p.141-150; S. Clay, «Les réactions du Midi : conflits, continuités et violences ", p. 55.

31. Arch. mun. de Bollène, $1 \mathrm{D}$ 3, délibération municipale du 15 nivôse an IV.

32. O. Tort, «L'image du Midi sous la Restauration : variations autour du tempérament méridional », p. 436-453.

\section{RÉSUMÉS}

Dans le couloir rhodanien, la présence de comportements politiques antagonistes donne naissance à une géographie politique complexe, où cohabitent en contiguïté régionale des espaces politiques diamétralement opposés. La dégradation précoce de la conjoncture politique, à partir du printemps 1790, favorise l'apparition de lignes de fractures importantes qui s'enracinent durant toute la décennie révolutionnaire. Ce climat politique délétère et anxiogène génère des conditions propres à façonner un nouvel imaginaire politique, livrant une vision déformée et partisane de la région. Les adresses et discours des acteurs attestent l'existence d'un hiatus entre les réalités complexes d'une géographie politique à géométrie variable et les représentations qu'en ont les différents acteurs. Il s'agira de montrer comment cet imaginaire partisan constitue un élément important des stratégies politiques.

\section{AUTEUR}

\section{NICOLAS SOULAS}

Agrégé et docteur en histoire moderne, Centre Norbert Elias, équipe HeMOc, Avignon Université 


\title{
L'autorité coloniale entre réel et
} virtuel : moissons, transhumance, interdictions et transgressions en Algérie (1935-1950)

\author{
Isabelle Chiavassa
}

1 Ce travail conçoit comme un cas d'étude du réel et du virtuel les interdictions imposées aux nomades à estivage tellien, en Algérie. Dans ces sociétés agro-pastorales, cet élevage en système extensif existe depuis l'Antiquité; il est la clef de voûte de l'économie. Au Maroc, certaines tribus du Moyen Atlas font les mêmes déplacements saisonniers entre sommets et plaines. En Algérie, sur les marges du Sahara, les éleveurs sont contraints au nomadisme pastoral, à la transhumance dite achaba, avec moutons, chèvres et chameaux. Au sud de l'Atlas tellien se situe ce que Marc Côte nomme un vaste boulevard de plaines ${ }^{1}$ : les hautes plaines sud-oranaises, domaine des céréales et des steppes, et lieu de passage permanent au cours de l'histoire ${ }^{2}$.

Dès avant les Romains, cette transhumance était fortement institutionnalisée ${ }^{3}$. L'achaba se dirige vers ces hauts plateaux, vers le Tell en été, l'azzaba vers le Sahara en hiver. Les nomades ne se réfèrent pas à des limites physiques ou des frontières ${ }^{4}$. L'achaba ${ }^{5}$ part à la recherche des pâturages de ces hautes plaines ou hauts plateaux steppiques, poursuit parfois plus au nord, dans le Tell. Ces usages algériens sont mentionnés par Bernard Nantet $^{6}$ et Claude Treyer ${ }^{7}$. Xavier Yacono ${ }^{8}$ et Robert Capot-Rey ${ }^{9}$ avaient déjà examiné de manière approfondie les modalités du parcours de ces nomades, de cet estivage tellien ou steppique. Il conduit principalement vers le Sersou, dans le département d'Alger, des troupeaux composés désormais de moutons, beaucoup plus que de chameaux. Ces deux derniers ouvrages présentent l'avantage d'être contemporains des faits.

3 J'étudierai ici uniquement l'achaba dans le secteur géographique du Sersou de 1935 à 1950 environ. En 1900, l'Algérie comptait déjà presque 7 millions de moutons, 3 millions de chèvres et 193000 chameaux $^{10}$. 

d'Oran. Je le définis au sens du géographe Perrin ${ }^{11}$, comme une zone plus restreinte que le plateau du Sersou, mais bien plus large que la commune mixte de ce nom. C'est la large bande de hautes terres semi-arides, à 950 mètres d'altitude, insérée entre les montagnes du Tell et les territoires où pousse l'alfa ${ }^{12}$, allongée entre le Djebel Nador et l'Ouarsenis, mais incluant les hauteurs sud de l'atlas tellien, zone agricole où des forêts poussent, où le blé dur et les lentilles sont cultivés. En effet, les troupeaux parvenaient bien jusque-là, ils ne restaient pas cantonnés dans la zone aride de l'alfa ${ }^{13}$. Mon interrogation porte sur le contrôle de l'achaba par l'administration coloniale : est-il réussite ou échec, réel ou virtuel ? J'ai exploré sur ce point les fonds d'archives des préfectures, sous-préfectures et communes mixtes conservées aux Archives nationales d'outre-mer ${ }^{14}$. Le contexte administratif et historique est celui d'une situation coloniale, offrant des institutions analogues à un département français : préfet et souspréfet, incluant toutefois en sus les communes mixtes, territoires où les Européens étaient largement minoritaires, sans maire élu, administrées par un administrateur fonctionnaire de l'État. La commune mixte est donc un territoire analogue, pour simplifier, à un arrondissement.

Pour l'arrière-plan théorique, je m'inspire d'un champ de recherche apparu pour l'époque moderne. Héloïse Hermant, en introduction à un ouvrage paru en $2016^{15}$, a décrit ce champ de recherche où les historiens de l'Europe moderne s'interrogent sur l'inflexion des cadres de la domination, sur le face-à-face entre populations et agents de l'État, agents surtout subalternes. Quand le consentement à la légitimité des mesures n'est pas acquis, il y a des refus de la contrainte, des contournements de la norme à la barbe des autorités, des zones de friction, une poussière d'actions de résistance passive, des jeux de négociation entre gouvernants et gouvernés, de la tolérance, du pragmatisme, ou encore du laissez-faire, des dérives. C'est dans cette optique que je vais aborder la norme de l'achaba fixée par l'État colonial, puis l'écart entre cette norme virtuelle et le réel ; en dernier lieu, j'évoquerai les moyens que choisit l'État pour faire face au contournement.

\section{Le virtuel, la norme : maîtrise de l'espace et du temps par l'autorité coloniale}

Le Sersou a toujours été la zone d'estivage de deux grandes tribus du Sud, les Larbaa et les Aït Otba. Ces deux tribus du Sud saharien, de Djelfa et de Laghouat ne sont pas les seules à venir chaque été au Sersou ${ }^{16}$ : des éleveurs emmènent aussi leurs troupeaux « du nord $»^{17}$ et surtout de l'est, de Chellala, Aïn Boucif, Bou Saada, Boghari et Djebel Nador, car même en ces lieux, ils sont confrontés à la sécheresse. La commune mixte de Chellala est zone de passage pour ceux qui viennent du sud et du nord, et fournit ellemême des troupeaux en transhumance. Tous ces troupeaux s'installent surtout à Trumelet, Burdeau, Victor-Hugo, Vialar et Bourbaki (fig. 1). 
Fig. 1. - Le Sersou, région de transhumance au sud des départements d'Alger et d'Oran. Échelle : $1 / 420000$.

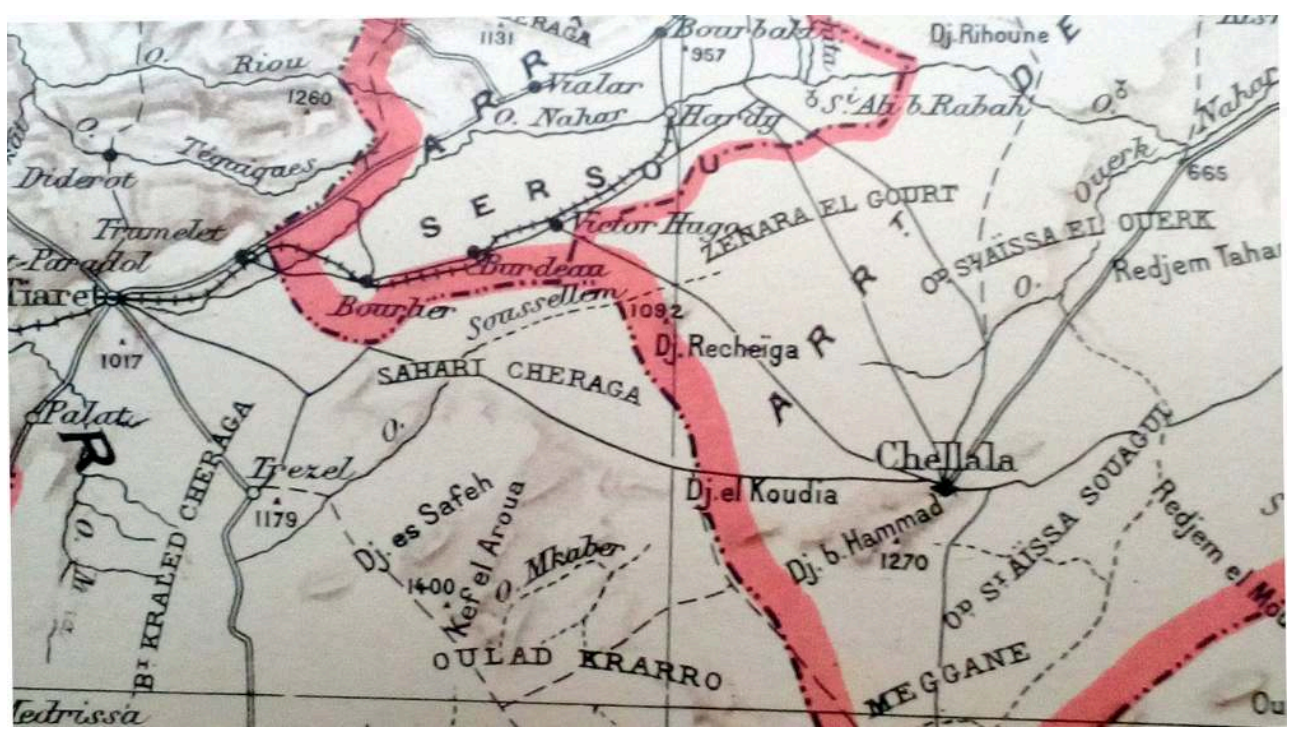

Arch. nat. outre-mer, cliché I. Chiavassa, D.R.

7 Si le Sersou attire, c'est qu'on y produit du blé et des lentilles. Les nomades ont une sorte de servitude acquise héritée du Sénatus-consulte de 1865, mais en l'absence de terre libre pour pâturer au Sersou ${ }^{18}$, ils louent les chaumes aux colons. Ils viennent donc après les moissons, et en nombre massif : en 1937, 38000 personnes, plus de 400000 ovins et 25000 chameaux font le déplacement. Ce système présente trois avantages : les nomades font pâturer leurs bêtes sur les chaumes; ils fournissent de la main-d'œuvre aux colons pour désherber et arracher les lentilles, pour récolter les céréales et pour les transporter sur leurs chameaux ; ils glanent, achètent des grains et emportent donc avec eux des provisions pour l'hiver.

L'autorité coloniale tente d'organiser, d'encadrer, de répartir, de rythmer ces parcours dans le temps et dans l'espace. Elle fixe la norme en définissant deux zones permanentes, la zone d'attente et la zone colonisée, et chaque année un calendrier constitué de deux dates simples, la date à partir de laquelle les troupeaux sont autorisés à arriver en zone d'attente, et celle d'installation en zone colonisée ${ }^{19}$, la logique étant de faire patienter jusqu'après la fin des moissons. Cette norme s'inscrit dans un règlement du gouverneur général de l'Algérie des années 1920, des circulaires du même aux préfets et surtout dans un arrêté annuel du préfet d'Alger, qui au préalable a consulté les deux sous-préfets concernés, ceux de Médéa et de Miliana. C'est toujours en avril ou mai que le préfet signe cet arrêté : en 1938, la date se fixe au 20 juin pour entrer en zone d'attente, puis au 10 août pour entrer en zone colonisée; de 1941 à 1943 , c'est toujours le 20 juillet et le 15 août; en 1944, c'est plus précoce, les 5 juillet et 5 août; encore plus tôt en 1946, dès les 15 mai et $1^{\text {er }}$ août ; et en 1947 , les $1^{\text {er }}$ mai et $1^{\text {er }}$ août ${ }^{20}$. Il faut observer que ces archives font entendre la voix des fonctionnaires proches du terrain, sous-préfet et administrateur, mais ne donnent pas accès à la parole, à l'opinion des tribus ni des éleveurs. 


\section{La réalité : un écart entre réel et virtuel}

9 Le constat, c'est le contournement de cette interdiction, la transgression des tribus et des éleveurs, qui arrivent bien avant la date autorisée, en zone d'attente et aussi en zone colonisée. En 1937, en raison de la sécheresse, ils commencent à arriver dès février (en revanche, ils repartent plus tôt que prévu, dès septembre) ; en 1941, ils sont en bordure de zone dès le 20 mars ; en 1942, ce sont les troupeaux qu'on dit à l'époque du nord, venus en fait de l'est, qui se ruent avant la fin de la moisson; en 1943, ils sont à Chellala en zone d'attente dès le début avril; en 1944, en mai, et ils se montrent agressifs, ne tolèrent pas les remontrances formulées par les caïds ; en 1945, ils arrivent du sud et du nord dès le $1^{\mathrm{er}}$ mai ; en 1947, ils commencent à arriver fin mars ${ }^{21}$.

10 Or aucune pénalité n'est prévue, et il y a 250 kilomètres de "frontière » ou plutôt de limite théorique, non physique, à surveiller pour freiner leur arrivée précoce. Il faut imaginer des troupeaux importants: rappelons les 400000 moutons et 25000 chameaux cités en $1937^{22}$. D'autres écueils surgissent dans le réel : les colons mettent trop tôt en location leurs chaumes, en pleine période d'interdiction (1942) ; les nomades justifient leur arrivée devant l'autorité par divers prétextes, alléguant une attestation fournie par la commune mixte de Chellala, ou un contrat de location de chaumes $^{23}$. Ceux dits «du nord» n'ont pas de chefs et se montrent plus indisciplinés que ceux venant du sud ${ }^{24}$. Les nomades causent de graves déprédations : $10 \%$ de la future récolte de blé est perdu en raison de leur piétinement en bord de route en 1945, dès le 25 mai. Ils épuisent aussi les chaumes par leur simple passage. Ils font du commerce de grains en fraude en 1942. Ils viennent en effet autant pour rapporter des grains sur leurs terres que pour faire pâturer les troupeaux.

Ils volent des gerbes et pillent, notamment en 1946, année exceptionnelle dans la mesure où ils sont venus sans moutons, mais au nombre de 40000 ou 45000 hommes. Cette année-là, l'administration n'ayant pas disposé de goumiers pour surveiller, ils volent pour se nourrir et pour emporter des provisions ; l'administrateur de l'achaba ne dispose même pas d'un véhicule de type "jeep " pour le contrôle. Certains campent près des fermes des colons européens. En 1947, ils sont là fin mars; à nouveau ce sont surtout des hommes et non des troupeaux, et l'administrateur écrit :

«Ce sont des miséreux sans provisions et sans argent, ils viennent pour travailler,

acheter, glaner et voler."

12 Or, ils ne pourront pas travailler ni glaner, le travail étant mécanisé par des moissonneuses-batteuses, il y aura peu de blé à acheter, reste donc la possibilité de voler, écrit l'administrateur, qui sait qu'en 1946, l'année précédente, ils ont parfois moissonné directement le blé à récolter, au lieu de se contenter des chaumes, et dérobé des gerbes sur des meules la nuit, devant les spahis impuissants à empêcher ces vols ${ }^{25}$.

13 Résultat de ces vols et pillages, des incidents et des rixes se produisent; un coup de revolver fut tiré en 1937. Autre point délicat, le besoin de main-d'œuvre temporaire disparaît : en 1936, les colons européens transportent déjà leurs récoltes par camions et n'ont plus besoin du transport par chameaux, sauf exceptionnellement en raison de la pénurie en 1944. 


\section{L'État colonial s'adapte}

14 Quels sont en conséquence les voies et les moyens de l'autorité pour réprimer la transgression? L'autorité reconnaît le contournement de la sphère normative. Une police de la transhumance s'organise, combinant une obligation pour les chefs de tribu d'accompagner leur tribu, et la mise en place par l'autorité coloniale d'un encadrement: en 1937, il est souhaité que les chefs de troupeaux se déclarent au préalable, et même que leur soit imposé de détenir une carte d'identitée ${ }^{26}$.

Deux fonctionnaires d'État ${ }^{27}$, désignés pour contrôler l'achaba durant quelques mois ${ }^{28}$, font des comptes rendus bimensuels et un rapport de fin de mission. Mais, surtout, on affecte à la surveillance les caïds ${ }^{29}$, les gardes champêtres, des cavaliers, des goumiers, des moghaznis (sept seulement en 1937), toutes sortes d'hommes montant à cheval ${ }^{30}$, et même des spahis. Par ailleurs, la gendarmerie est déjà présente. En 1947, l'état-major de la $\mathrm{X}^{\mathrm{e}}$ région militaire organise des détachements motorisés armés ${ }^{31}$. On institue aussi des points de contrôle aux six lieux principaux par lesquels arrive la transhumance: ces endroits sont de Foucauld, Belkitar, Chabounia, Bourbaki, Hardy et Victor-Hugo. Tout cet encadrement donne lieu à des rapports sur l'achaba qui parviennent au préfet et au gouverneur général; on récompense les caïds et gardes champêtres efficaces, on sévit s'ils n'assurent pas leur fonction de contrôle. L'autorité a aussi recours au judiciaire pour les pillages ou vols, mais les nomades décampent avant la réaction du juge de paix.

16 L'autorité coloniale choisit l'anticipation : le préfet demande en amont aux sous-préfets le nombre d'arrivants (ainsi en 1944, on prévoit 13600 ovins et 450 chameaux au Sersou $^{32}$ ), ainsi que le nombre d'ouvriers agricoles qui seront nécessaires. S'il anticipe une sécheresse exceptionnelle ou des nuages de sauterelles qui vont s'attaquer aux récoltes, le gouverneur général alerte le préfet d'Alger qui choisit alors une date plus précoce d'arrivée (le mois de mai). Ainsi, en mars 1937, il demande au préfet l'indulgence, la tolérance ${ }^{33}$; à nouveau, en raison de la sécheresse qui sévit au sud, à Laghouat. En juin 1942, il sollicite le préfet, et plusieurs communes mixtes acceptent de recevoir qui 20000 , qui 25000 moutons sur leur territoire ${ }^{34}$. En 1944, des arrivées massives et prématurées de troupeaux sont prévues, toujours pour cause de sécheresse et de sauterelles, le préfet prévient les sous-préfets ${ }^{35}$. C'est la souplesse, le réalisme qui dominent. En 1943, le commandant militaire de Ghardaia prend les choses en main : il réunit fin mars le sous-préfet de Médéa et les administrateurs de communes mixtes. Cette réunion aboutit à décider pour cette année une séparation entre tribus, entre les Ouled Naïls, que l'on va contraindre à s'en retourner, et les Larbaa qui seront admis en zone d'attente, mais seulement en trois lieux (ils sont plus de 80000 hommes), et que l'on contraindra à repartir chez eux si d'aventure les pluies arrivent ${ }^{36}$.

17 L'administrateur de l'achaba se comporte et se désigne dans ses rapports en conciliateur, en médiateur. Ainsi en 1941, pour favoriser l'achat de grains de blé, les ouvriers sont payés (en partie) en bons d'achat ${ }^{37}$. Les colons eux-mêmes font preuve de tolérance: en 1937, ils sont compréhensifs, charitables face à la misère des nomades, sauf s'ils approchent de leurs vergers, de leurs jardins cultivés, ou des chaumes réservés qui servent au troupeau propre du colon. À nouveau, en 1947, ils se montrent patients ${ }^{38}$. C'était déjà l'avis de l'administrateur de l'achaba de 1937 : «il faut accorder les intérêts opposés »; c'est encore en 1947 l'avis de son successeur, dans son rapport de fin de mission très réaliste. 

contournent et transgressent sans vergogne les interdictions, utilisent la stratégie du fait accompli : c'est l'ineffectivité de la norme. La légitimité de la contrainte n'est pas totalement admise par les nomades, mais l'autorité coloniale ne demeure pas dans le virtuel. Elle s'inscrit bien dans le réel, désignant l'achaba comme " un mal nécessaire " en $1942^{39}$, elle s'adapte. D'ailleurs, dès 1938 elle était bien consciente de ses faiblesses : on écrivait « l'administration essaie de réglementer cette migration régie par des lois naturelles et les nécessités géographiques ${ }^{40}{ }$; et parfois, l'autorité coloniale adopte une attitude de résignation complète. "Il faudrait un régiment », écrit-elle en 1937, il faut entendre par là pour canaliser les arrivées massives ${ }^{41}$. Cette année-là, dès le mois d'avril, les nomades ont fait pacager non les chaumes mais bien les récoltes, et couper $\mathrm{du}$ blé pour leurs chevaux. Ils continuent à le faire en présence des gendarmes, et déclarent que l'on peut bien leur dresser tous les procès-verbaux que l'on voudra ${ }^{42}$. L'autorité coloniale écrit en 1942 :

«Il est vain de fixer des dates, car ils arrivent avant la fin de la moisson... C'est

l'anarchie, et on se borne à éviter les incidents sanglants ${ }^{43}$. " bien connus, par exemple le refus du calendrier révolutionnaire en 1793, mais en situation coloniale, l'attitude de crainte de l'État et des colons m'inspirera plutôt une comparaison avec les travaux d'Ann-Laura Stoler sur la colonisation hollandaise en Indonésie ${ }^{50}$. Ces recherches ont souligné la grande angoisse du colonisateur pour sa sécurité, les hésitations et tâtonnements des administrateurs locaux; là aussi, le colonisateur ne parvient jamais à tout contrôler. Les documents témoignent de cette crainte, à tel point qu'Ann-Laura Stoler considère les archives en soi non pas seulement comme source, mais comme sujet d'étude et comme terrain d'ethnographie. En Algérie, au Sersou, l'urgence consiste à protéger les récoltes. Le caractère inefficace et dérisoire des mesures prises par l'État colonial, mesures limitées à des arrêtés préfectoraux, au recours aux juges de paix ou à quelques hommes à cheval, montre la difficulté du colonisateur à imposer sa norme. 


\section{BIBLIOGRAPHIE}

CAPOT-REY Robert, Le Sahara français, Paris, Presses universitaires de France (Pays d'outre-mer, 4e série), 1953.

CÔTE Marc, « L'Algérie plurielle », L'information géographique, nº 4, 2001, p. 340-351.

FAURE Patrice, LEVEAU Philippe, « Les marges de la Numidie romaine à la lumière d'une nouvelle inscription des monts des Ouled Naïl », Antiquités africaines, $\mathrm{n}^{\circ}$ 51, 2015, p. 119-142.

HADEID Mohamed, BENDJELID Abed, FONTAINE Jacques, oRMAUX Serge, « Dynamique spatiale d'un espace à caractère steppique : le cas des Hautes Plaines sud-oranaises (Algérie) ", Cahiers de géographie du Québec, vol. 59, nº 168, décembre 2015, p. 469-496.

HERMANT Héloïse (dir.), Le pouvoir contourné : infléchir et subvertir l'autorité à l'âge moderne, Paris, Classiques Garnier, 2016.

LARCHER Émile, Traité élémentaire de législation algérienne, 3e éd., Paris, Rousseau, 1923.

NANTET Bernard, Histoire du Sahara, Paris, Tallandier, 2015.

PERRIN R., « Le Sersou. Étude de géographie humaine (Premier article) », Méditerranée, $1^{\mathrm{er}}$ année, $\mathrm{n}^{\circ} 2-3,1960$, p. 61-118.

STOLER Ann-Laura, Au cour de l'archive coloniale : questions de méthode, Paris, Éditions de l'EHESS, 2019.

TREYER Claude, Sahara 1956-1962, Paris, Les Belles-lettres, 1966.

YACONO Xavier, Les bureaux arabes et l'évolution des genres de vie indigènes dans l'Ouest du Tel algérois, Paris, Larose, collection de documents inédits et d'études sur l'histoire moderne et contemporaine de l'Algérie, $3^{\text {e }}$ série, Études, 1, 1953.

\section{NOTES}

1. M. Côte, « L’Algérie plurielle », p. 340, 341.

2. M. Hadeid, A. Bendjelid, J. Fontaine, S. Ormaux, «Dynamique spatiale d'un espace à caractère steppique : le cas des hautes plaines sud-oranaises (Algérie) », p. 471, 473, 474.

3. P. Faure, Ph. Levreau, "Les marges de la Numidie romaine à la lumière d'une nouvelle inscription des Monts des Ouled Naïls », p. 138.

4. En 1927, le gouverneur général a réglementé l'achaba; les colons ne louent leurs chaumes qu'en passant par l'administration.

5. Migration saisonnière selon des itinéraires fixés depuis au moins le xxe siècle (décrits par Émile Masqueray).

6. B. Nantet, Histoire du Sahara.

7. Cl. Treyer, Sahara 1956-1962, p. 22.

8. X. Yacono, Les bureaux arabes et l'évolution des genres de vie indigènes dans l'Ouest du Tell algérien, p. 41, 45, 49, 355-356.

9. R. Capot-Rey, Le Sahara français. 
10. É. Larcher, Traité de législation algérienne, p. 97.

11. R. Perrin, «Le Sersou. Étude de géographie humaine », p. 61, 63.

12. Et aussi des plantes halophiles, des graminées, des jujubiers.

13. Ils vont notamment à Trumelet (qui se situe dans la commune mixte de Tiaret), ou dans des communes de plein exercice.

14. Aix-en-Provence.

15. H. Hermant (dir.), Le pouvoir contourné. Infléchir et subvertir l'autorité, Introduction.

16. Cette transhumance combine location de terrains et droit d'estivage des tribus, comparable à une servitude.

17. C'est le terme employé dans les archives.

18. Il existe au Sersou peu de terre " arch » ou de terre domaniale.

19. Il existe un cas similaire : déjà en 1904, l'État a tenté d'imposer des lieux et des dates pour la cueillette de l'alfa, distinguant nettement en théorie le Tell et les Hauts Plateaux, mais a dû les modifier dès 1911, par simple réalisme (cote FR ANOM 912/227).

20. Cote FR ANOM $912 / 486$.

21. Ibidem.

22. Ibidem.

23. Par ailleurs, comme ils savent que les troupeaux locaux du Sersou, eux, sont bien admis à Chellala l'hiver en transhumance, ils en font un argument pour exiger d'entrer l'été, au moins à Chellala en zone d'attente (FR ANOM 912/486).

24. FR ANOM 912/486.

25. FR ANOM 912/227.

26. FR ANOM $912 / 486$.

27. Des administrateurs des services civils adjoints.

28. Ils sont d'ailleurs contraints d'utiliser leur voiture personnelle pour ce travail.

29. Qui rendent compte à leur administrateur de commune mixte.

30. FR ANOM 912/486.

31. FR ANOM 912/227.

32. FR ANOM 912/486.

33. FR ANOM $912 / 486$.

34. FR ANOM 912/486.

35. FR ANOM 912/227.

36. FR ANOM 912/486.

37. FR ANOM 912/486.

38. FR ANOM $912 / 227$.

39. FR ANOM 912/486.

40. FR ANOM 912/cote.

41. FR ANOM 912/486.

42. FR ANOM 912/486.

43. FR ANOM 912/486.

44. FR ANOM 912/486. 
45. FR ANOM 912/227.

46. Il est enfin suggéré en 1947 de fixer une date plus réaliste, car l'administration est incapable de la faire respecter (FR ANOM 912/227).

47. Le jeu est plus subtil et complexe qu'il n'y paraît face à la transhumance, car certains colons européens envoient eux aussi leurs troupeaux au Sersou. Il y a échange de pâturages, été contre hiver, entre Chellala et le Sersou. Certains troupeaux appartiennent par moitié à des propriétaires des deux territoires; ce sont de gros propriétaires spéculateurs (FR ANOM 912/486). De plus, le jeu met en scène plusieurs acteurs : l'État colonial, les tribus, les responsables indigènes chargés de surveiller les migrations, les colons européens et également certains indigènes qui possèdent euxmêmes des terres de pâturage ou des troupeaux.

48. FR ANOM 912/227.

49. FR ANOM $915 / 96$ et FR ANOM 91/1K419.

50. A.-L. Stoler, Au coeur de l'archive coloniale, passim.

\section{RÉSUMÉS}

L'autorité administrative a coutume de prescrire, d'interdire, de fixer des limites. Dans la réalité, ces injonctions restent dénuées d'effet. Un cas particulier de ce décalage entre virtuel, prescrit par l'État, et réel, constaté dans une Algérie colonisée fait l'objet de l'article, à partir de fonds des Archives nationales d'outre-mer. L'administration coloniale (gouverneur général, préfet, souspréfet) gère les espaces et le temps des transhumances du Sud et de l'Est algérien vers le Nord, où des tribus emmènent leurs troupeaux d'ovins et de chameaux à la recherche des hautes plaines, de mars à juin, avant le retour en septembre. Elle tente d'encadrer cette transhumance et de protéger les cultures des ravages causés par les troupeaux. Ces injonctions ne sont pas toujours respectées. Les moyens qu'elle utilise restent inefficaces : exemple typique d'un cas général, celui des difficultés rencontrées sur le terrain par l'administration coloniale, celui de l'ineffectivité de la norme.

\section{AUTEUR}

\section{ISABELLE CHIAVASSA}

Conservateur en chef du patrimoine, Ministère des armées, Direction des patrimoines, de la mémoire et des archives 


\title{
Conclusion
}

\author{
Jean Soumagne
}

1 Mondes réels et mondes virtuels tendent en ce début du XxI ${ }^{\mathrm{e}}$ siècle à se mélanger dans la vie quotidienne. Au fur et à mesure que les mass media ont pris de l'ampleur, chaque habitant de la planète a vu s'élargir ses horizons en devenant plus ou moins familier de terres et de populations qui lui étaient relativement étrangères auparavant; l'« instantanéité » de l'information « en direct », via les châ̂nes d'information continue et les réseaux sociaux, a envahi les esprits au rythme des « écrans » démultipliés et des «temps de cerveau disponibles» dans les tiers-lieux et les «non-lieux» des mégalopoles. Ainsi a surgi l'impression d'un «réel» omniprésent: réel parce que photographié, réel parce que filmé, les images étant réputées être l'assurance de la véracité.

2 Cette impression s'est trouvée remise en cause, la "société du spectacle " telle qu'analysée par les sociologues et les philosophes pouvant aisément glisser vers une société de tromperie, d'" infox » tant par la substitution dans le temps et dans l'espace des images que par leurs modifications électroniques, ou encore par les effets disproportionnés de leur ressassement, l'accumulation ou le matraquage annihilant tout esprit critique.

3 Ainsi, des faits réels mineurs imposent une vision déformée de la réalité du monde ; des éléments fragiles, des "signaux faibles", c'est-à-dire simplement virtuels - en puissance - peuvent apparaître dans l'esprit des personnes réceptrices comme des témoignages fiables d'une réalité générale. Les potentialités deviennent alors des faits ou des objets reconnus universellement; le possible devient le probable, le probable se mue en authentique. De fait, l'infusion générale d'informations erronées devient aujourd'hui une technique courante de manipulation, de soft power de la part d'individus, de groupes, de sectes, d'États, tous cherchant à influencer les opinions par la croyance dans des vérités alternatives émergentes.

4 La multiplication des appareils électroniques, l'invasion du numérique présentent un autre visage, celui de l'explosion fictionnelle à travers les œuvres cinématographiques au sens large, non seulement les films et les documentaires mais les innombrables « séries"; celles-ci offrent à tout instant sur les tablettes et les smartphones des opportunités d'entrer dans un monde où l'imaginaire prend souvent appui sur des 
réalités déjà existantes, ou sur des événements du passé, pour embrayer ensuite sur les utopies, les hétérotopies ou les uchronies. L'insertion de la fiction dans la réalité entraîne un mélange attirant, mais intellectuellement instable. La confusion des genres va au-delà de ce que les publireportages ou bien les documentaires tendancieux peuvent proposer, ceux-ci pouvant être décryptés et réfutés par une analyse méthodique démêlant le vrai du faux. Au contraire, les "autres mondes » fictionnels appartiennent largement à la création qui se veut artistique, et offrent une entrée quasi onirique à ces univers du futur ou du passé, ou d'un présent transformé. Ces mondes virtuels tirent de leur nature distractive un pouvoir matriciel sur l'esprit humain le faisant, temporairement, se détacher des contingences du quotidien. Ils ouvrent des perspectives, mais peuvent conduire à un affaiblissement de la distinction entre réel et imaginaire, faisant passer l'imaginaire pour du virtuel. Ce possibilisme exacerbé - rien n'est impossible -, croisé avec un individualisme forcené, peut aller de pair avec un scepticisme débridé vis-à-vis des réalités courantes. Le déni du réel ordinaire s'opère au profit du virtuel extraordinaire; il se conjugue avec la défiance à l'égard du discours des élites, remettant en cause l'exposé des scientifiques au profit des « explications non officielles ", d'éléments cachés, de "vérités » tues jusque-là et que révèlent de nouveaux gourous.

5 Alors, la submersion de l'imaginaire fait apparaître une vision du monde différente, déformée et refaçonnée, où l'homme peut se frayer un chemin hors des sentiers balisés par la Science et le Politique, répondant aux séductions du monde rêvé.

\section{AUTEUR}

\section{JEAN SOUMAGNE}

Professeur émérite des universités en géographie et aménagement urbain, membre du laboratoire Espaces et sociétés (ESO, UMR 6590, université d'Angers/CNRS) 